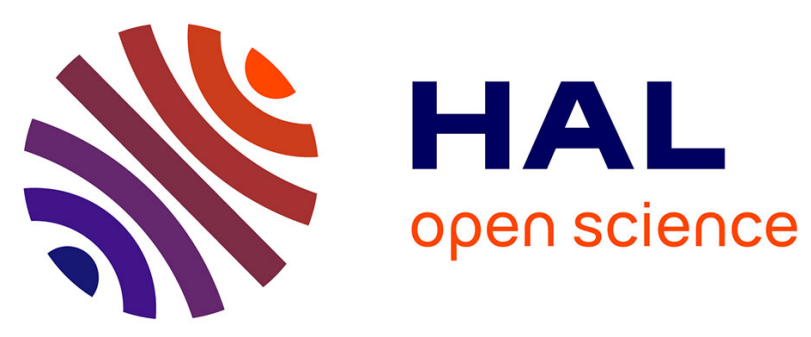

\title{
CAD/Analysis Integration
}

\author{
Abdelmajid Benamara
}

\section{To cite this version:}

Abdelmajid Benamara. CAD/Analysis Integration. Mécanique [physics.med-ph]. Ecole Nationale d'Ingénieurs de Monastir, 2006. tel-00434468

\section{HAL Id: tel-00434468 \\ https://theses.hal.science/tel-00434468}

Submitted on 23 Nov 2009

HAL is a multi-disciplinary open access archive for the deposit and dissemination of scientific research documents, whether they are published or not. The documents may come from teaching and research institutions in France or abroad, or from public or private research centers.
L'archive ouverte pluridisciplinaire HAL, est destinée au dépôt et à la diffusion de documents scientifiques de niveau recherche, publiés ou non, émanant des établissements d'enseignement et de recherche français ou étrangers, des laboratoires publics ou privés. 


\section{UNIVERSITE DE MONASTIR \\ ECOLE NATIONALE D'INGENIEURS DE MONASTIR

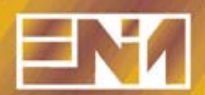

Laboratoire de Génie Mécanique MRSTDC-LAB-MA-05

\section{Rapport de Synthèse des Travaux de Recherche}

\section{Présenté pour l'obtention $d u$ :}

Diplôme d'Habilitation Universitaire en Génie Mécanique

Par:

\section{ABDELMAJID BENAMARA}

Juillet 2005 


\section{Table des matières}

Curriculum Vitae

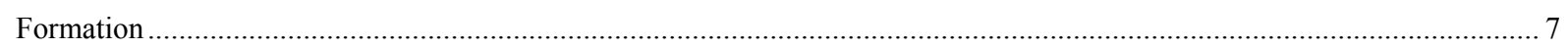

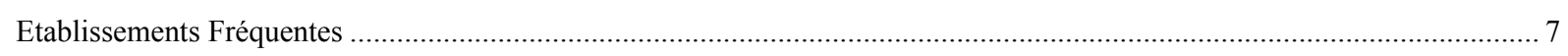

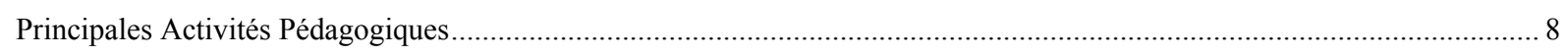

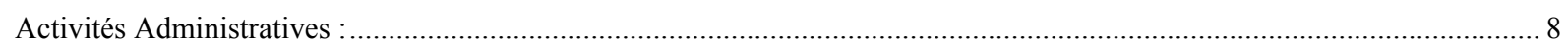

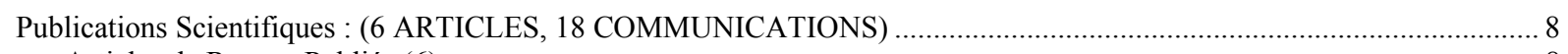

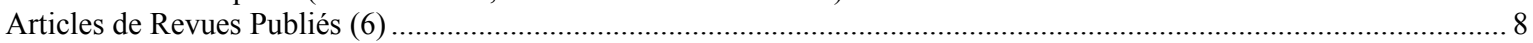

Articles de Revues Soumis : 2 ( 2005 )

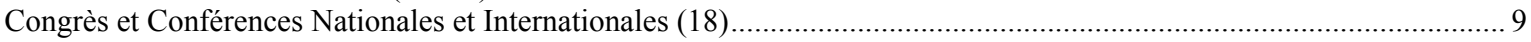

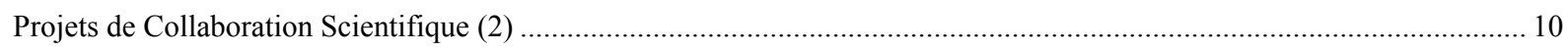

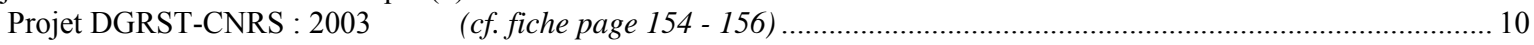

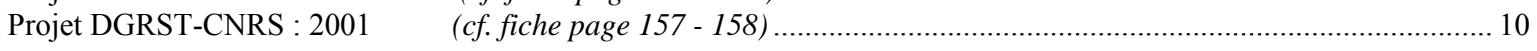

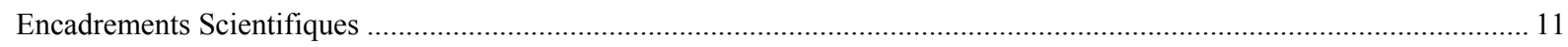

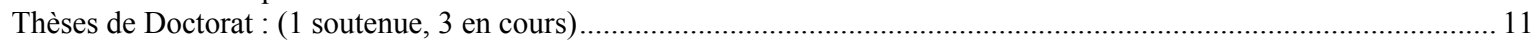

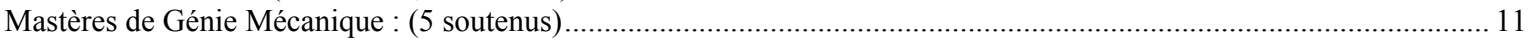

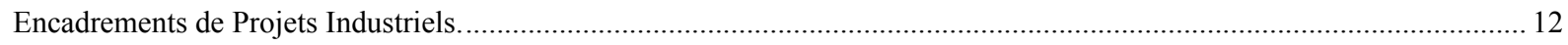

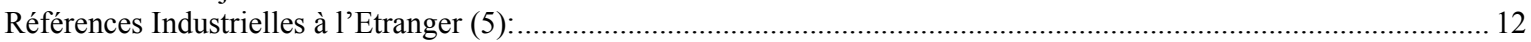

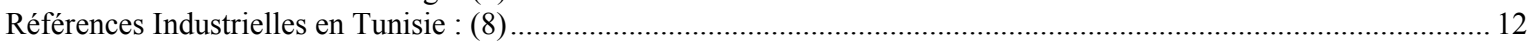

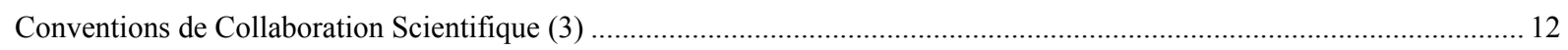

Participation à des Manifestations Scientifiques Nationales (7) et Internationales (13) ..................................................... 13

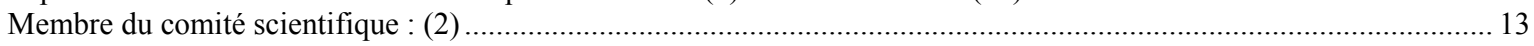

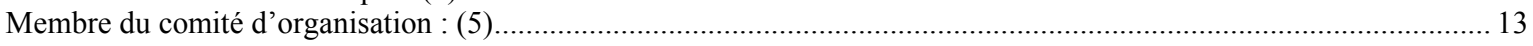

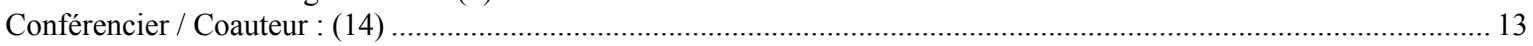

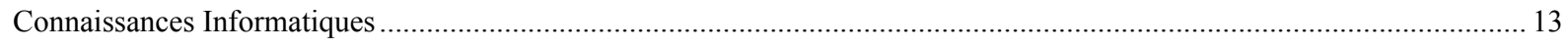

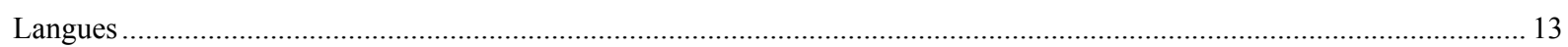

Introduction Générale....................................................................................................................... 14

PARTIE I : $\quad$ Introduction et mise en situation .............................................................. 16

1. INTRODUCTION ............................................................................................................................................................... 17

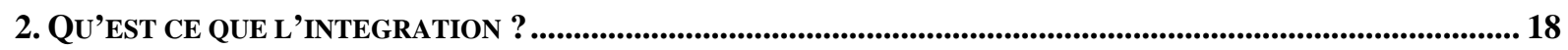

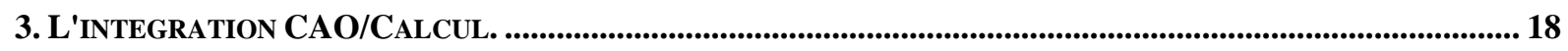

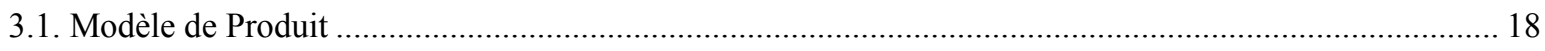

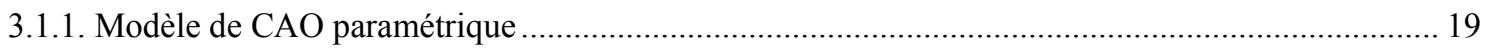

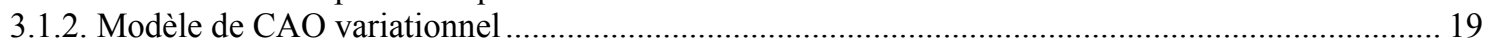

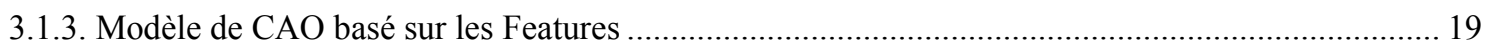

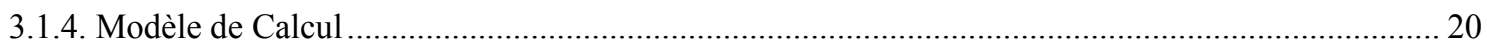

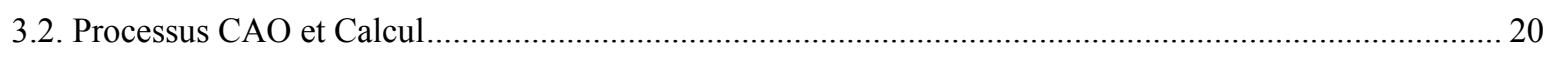

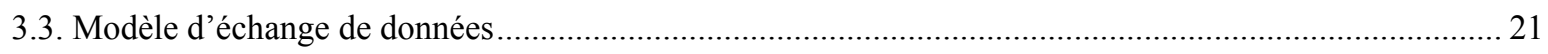

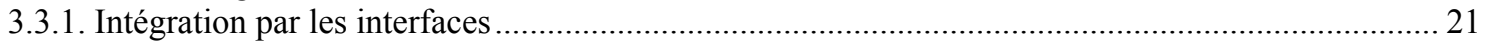

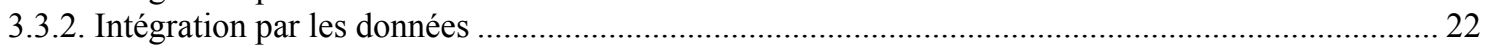

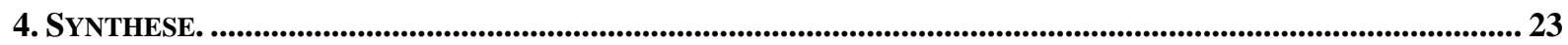


PARTIE II : $\quad$ Etat de l'art \& Positionnement des Travaux.................................................... 24

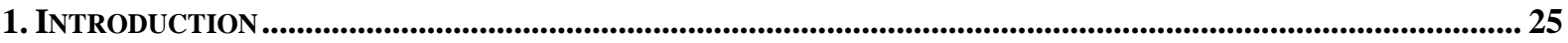

2. PRINCIPAUX PROJETS IMPLIQUANT DES LABORATOIRES DE RECHERCHE ET DES INDUSTRIELS .................... 25

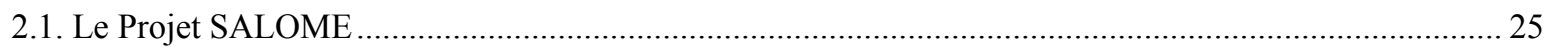

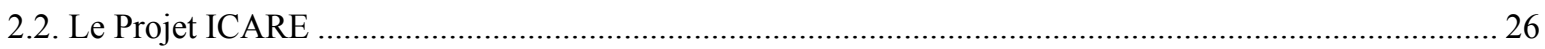

2.3. Le Projet Aide à la Conception d'une Chaîne Logistique (laboratoires LAG et GILCO) ........................... 26

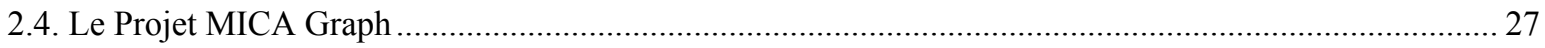

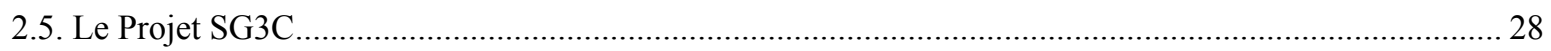

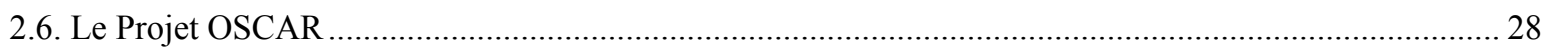

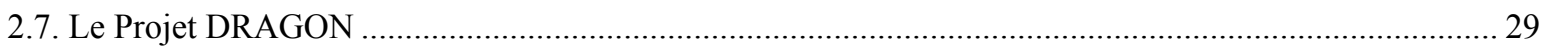

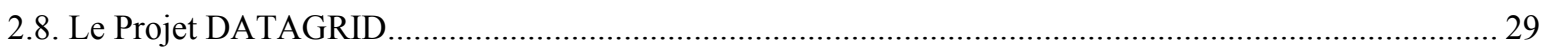

3. ETAT DE L’ART SUR LES APPROCHES D'INTEGRATIONS EXISTANTES .............................................................. 30

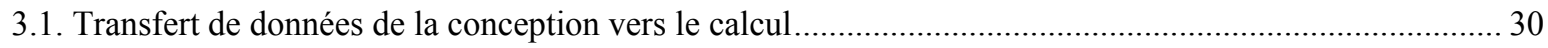

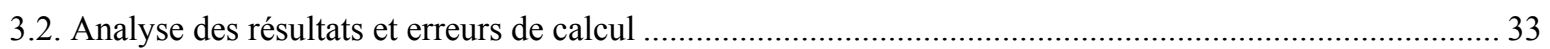

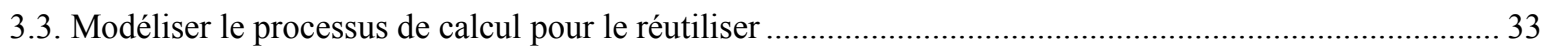

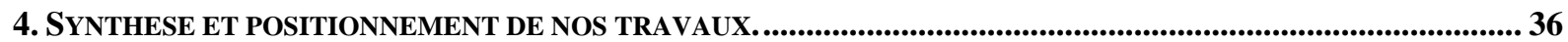

PARTIE III : $\quad$ Synthèse des Travaux de Recherche sur l’Intégration CAO/Calcul............ 38

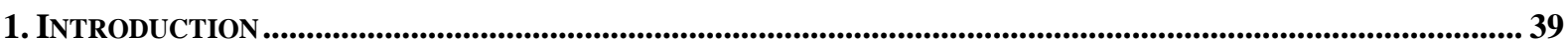

2. APPROCHE D'INTEGRATION CAO/CALCUL PAR ENRICHISSEMENT DU MODELE DE PRODUIT........................ 39

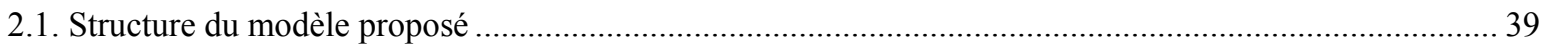

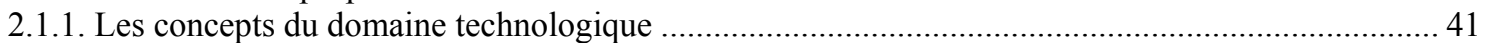

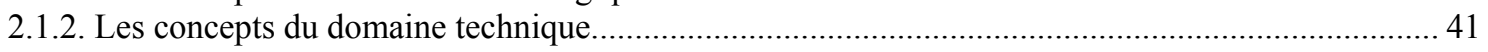

2.2. Mise en œuvre informatique : F-CAD, un prototype de Conception Fonctionnelle ..................................... 42

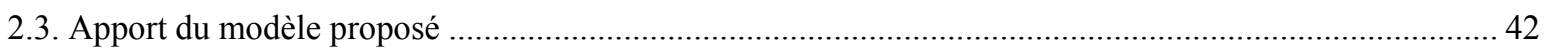

3. APPROCHE D'INTEGRATION CAO/CALCUL PAR FEATURES dE CALCUL. ................................................ 43

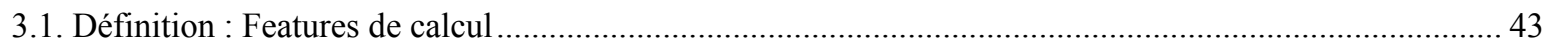

3.2. Organisation des features de calcul ....................................................................................................... 44

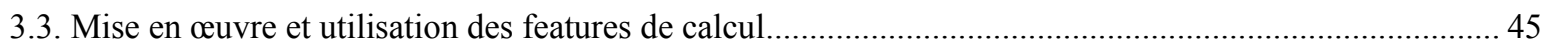

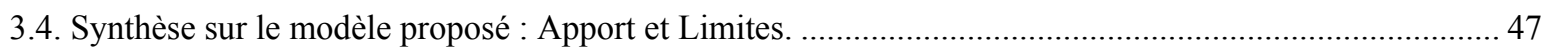

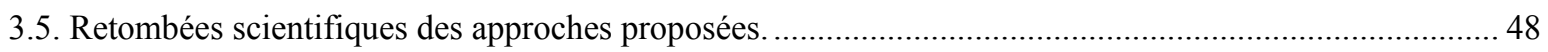

4. APPROCHE D'INTEgRation CAO/CALCUl PAR RECONSTRUCTION DU MODELE CAO A PARTIR DES

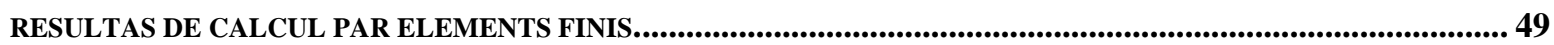

4.1. Hypothèses et éléments de base du modèle proposé ………........................................................................ 50

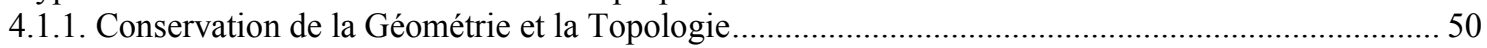

4.1.2. Changement de Géométrie sans changement de Topologie.......................................................... 51

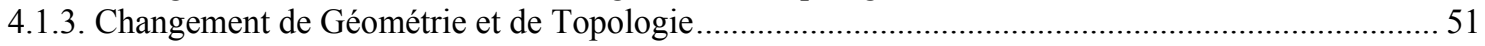

4.2. Algorithme général de reconstruction des modèles $\mathrm{CAO}$ à partir d'un maillage déformé ....................... 51

4.3. Reconstruction des Modèles déformés sans changement de géométrie et de topologie............................ 52

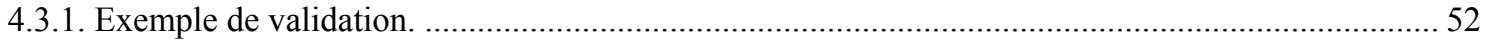

4.4. Reconstruction des modèles déformés avec changement de géométrie et sans changement de topologie. 53

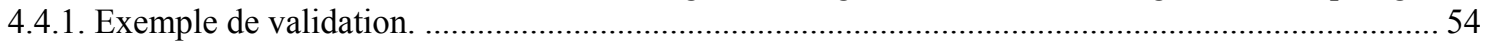

4.5. Reconstruction des modèles déformés avec changement de géométrie et changement de topologie......... 54

4.6. Algorithmes de reconstruction d'une surface à partir d'un maillage surfacique. .......................................5 55

4.6.1. Etat de l'art sur les méthodes de reconstruction de surfaces à partir d'un maillage..........................56 56

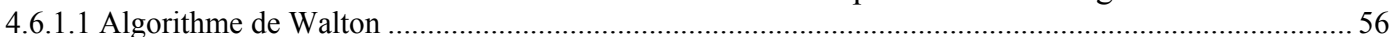

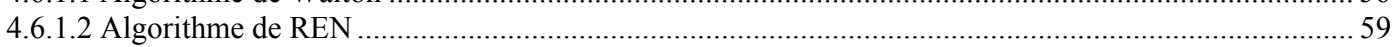

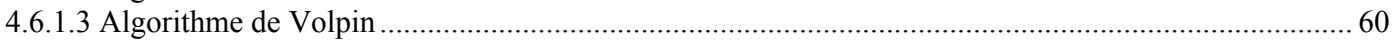

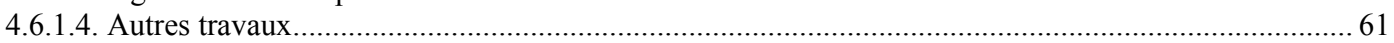


4.6.2. Algorithme proposé pour la reconstruction d'une surface à partir d'une triangulation. ................ 61

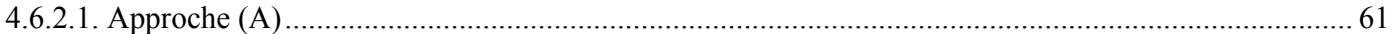

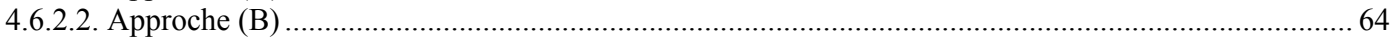

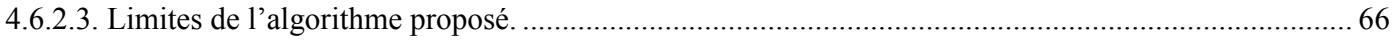

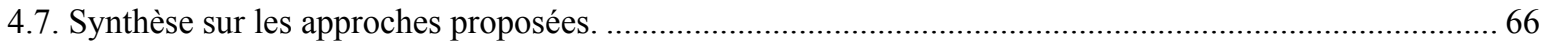

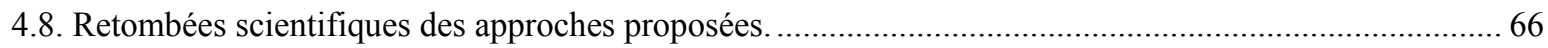

5. Plateforme de DEVELOPPEMENT DEDIEE A INTEGRATION CAO/CALCUL...............................................68

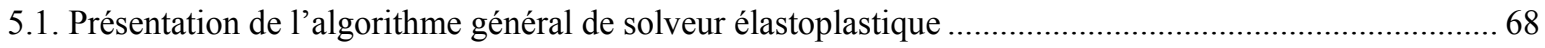

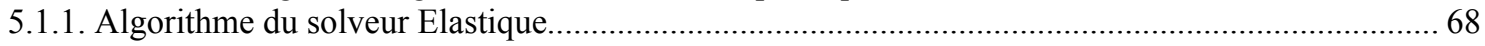

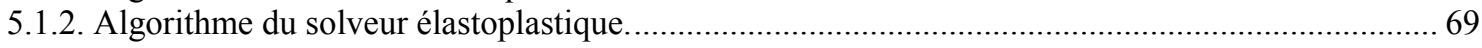

5.2. Exemple de validation et confrontation des résultats avec des codes de calcul industriels ....................70

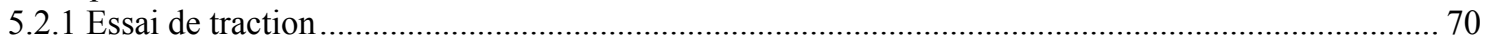

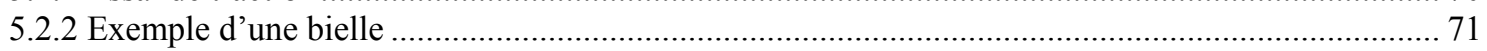

5.3. Avantages et limites du solveur élastoplastique proposé.................................................................. 72

5.4. Retombées scientifiques des approches proposées.................................................................. 72

PARTIE IV : $\quad$ Synthèses des Travaux de Recherche Emergeants ........................................... 73

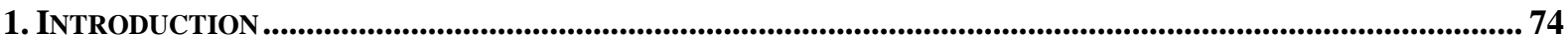

2. SIMULATION NUMERIQUE DU PROCEDE D’EMBOUTISSAGE DES TOLES MINCES........................................... 74

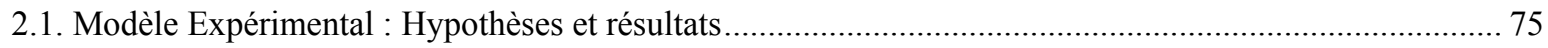

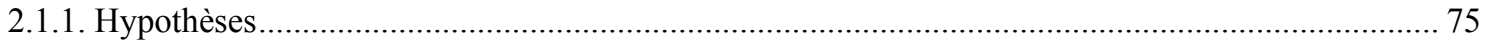

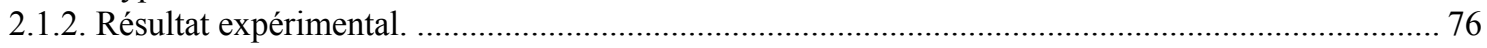

2.2. Modèle Numérique : Hypothèses et résultats ...................................................................... 76

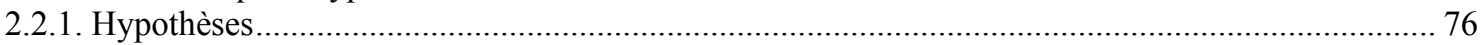

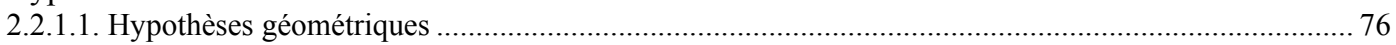

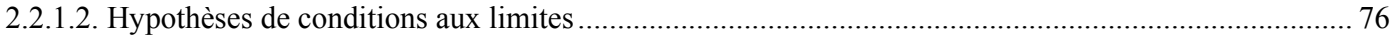

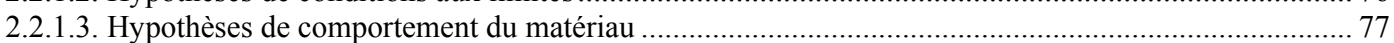

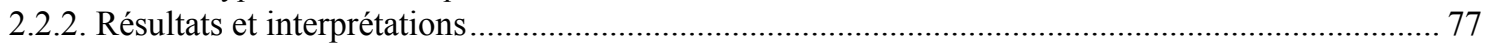

2.3. Retombées scientifiques des approches proposées....................................................................... 79

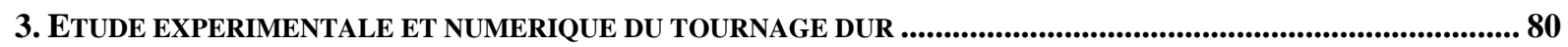

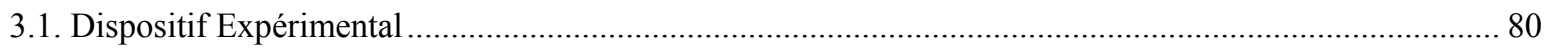

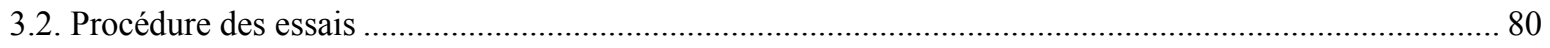

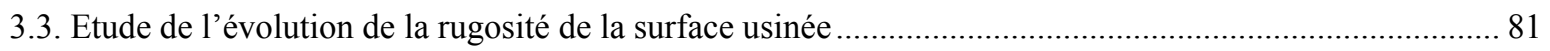

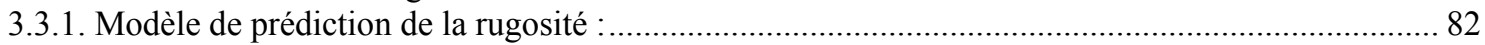

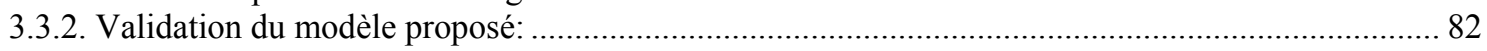

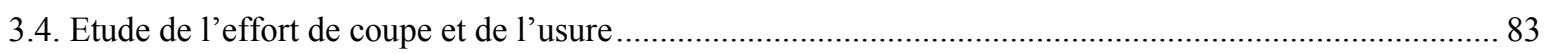

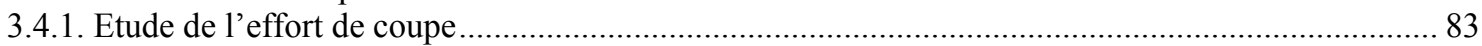

3.4.1.1. Evolution des efforts de coupe en fonction de la vitesse de coupe ................................................ 83

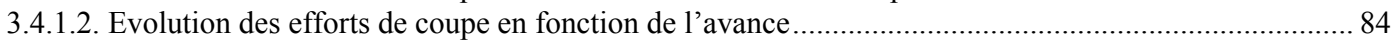

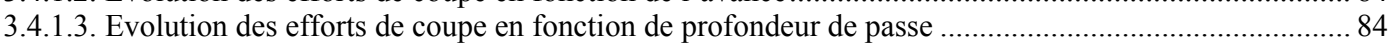

3.4.1.4. Prédiction des efforts en fonction des paramètres de coupe.......................................................... 85

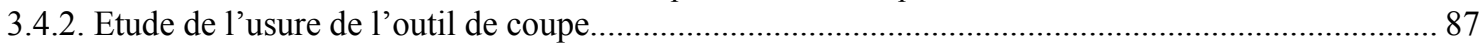

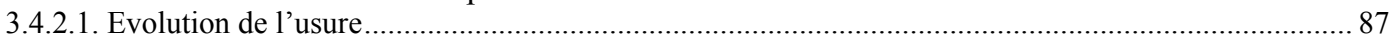

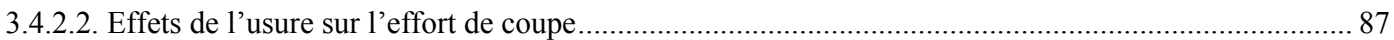

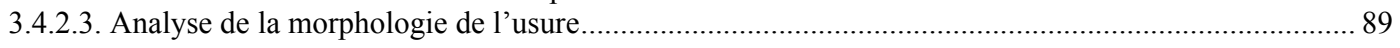

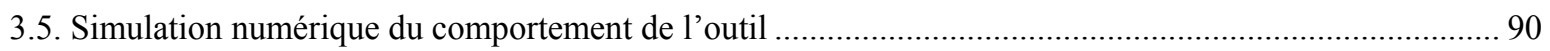

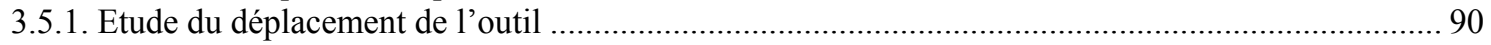

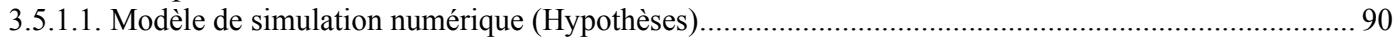

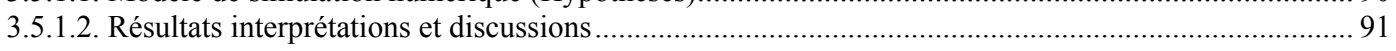

3.5.1.3. Effets des paramètres de coupe sur l'amplitude des déplacements ................................................ 93

3.6. Retombées scientifiques des approches proposées..................................................................95

4. MODELE DE CONCEPTION DE MOULES D’INJECTION PLASTIQUE BASE SUR LES FEATURES ..........................996

4.1. Etat de l'art : Conception des moules d'injection plastiques basée sur les features ..............................96

4.2. Features inhérentes au domaine de la conception des moules .............................................................97 


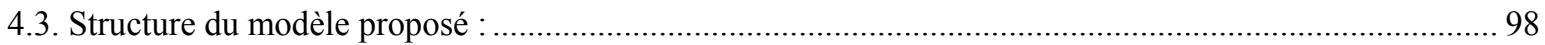

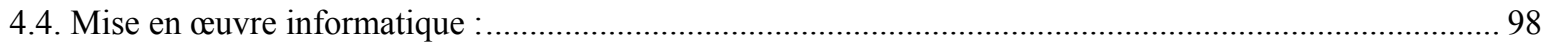

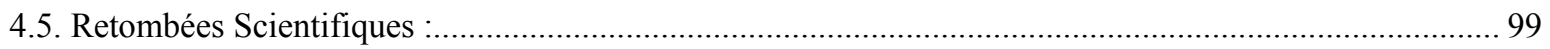

PARTIE V : Conclusions Générales et Perspectives................................................................ 100

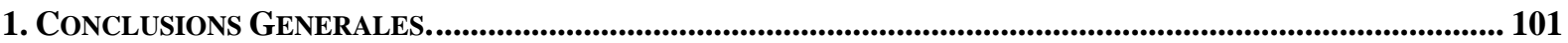

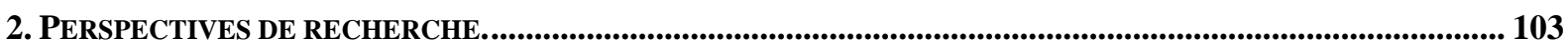

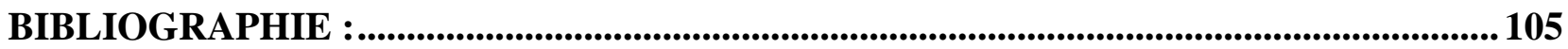

ANNEXE I : $\quad$ Fiches Publication Scientifique ................................................................................. 113

ANNEXE II ： Fiches Encadrement Scientifique ........................................................................137

ANNEXE III ： Fiches Projet de Collaboration Scientifique ............................................... 153 


\title{
Curriculum Vitae
}

\author{
Abdelmajid BenAmara \\ Né le : 10-11-1966 à Tataouine. \\ Marié, 3 enfants. \\ Adresse : ENIM, 5019 Monastir. \\ Tél : (+216) 98.43.80.07 \\ Abdel.BenAmara@enim.rnu.tn \\ http://www.benamara.fb.bz/ \\ http://www.lgm.rnu.tn
}

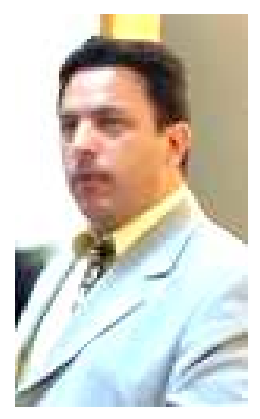

\section{FORMATION}

- Thèse de Doctorat, Génie Mécanique / Automatique Industrielle et Humaine, Décembre 1998. Régime : Cotutelle de thèse entre l'Ecole Nationale d'Ingénieurs de Tunis et l'Université de Valenciennes en France.

Sujet : Contribution à l'intégration de la composante calcul dans une démarche de conception fonctionnelle intégrée, application aux mécanismes.

- Diplôme des Etudes Approfondies, Génie Mécanique, Février 1992.

Ecole Nationale d'Ingénieurs de Tunis

Sujet : Intégration CAO/Calcul (Interface Pro/Engineer - Modulef).

- Maîtrise ès Sciences Techniques, Fabrication Mécanique, Juin 1990

Ecole Normale Supérieure de l'Enseignement Supérieur,

PFE : Conception et réalisation d'une voiture électrique.

- Certificat d'Aptitude à l'Enseignement Technique, CAET, Juin 1990

Ecole Normale Supérieure de l'Enseignement Supérieur.

\section{ETABLISSEMENTS FREQUENTES}

- ENIM : Ecole Nationale d'Ingénieurs de Monastir,

Maître Assistant de l'Enseignement Supérieur

Assistant de l'Enseignement Supérieur

Septembre 1999 -- présent Septembre 1993 - Septembre 1999

- EPT : Ecole Polytechnique de Tunisie,

Responsable Scientifique, Module Conceptique.

Septembre 2002 -- présent

- INSAT : Institut National des Sciences Appliquées et de Technologie, Vacataire, Module Analyse Fonctionnelle.

Décembre 2002

- UVHC : Université de Valenciennes et du Hainaut Cambrèsis,

Année 1997 --199 98 Vacataire, Module Conception Assistée par Ordinateur (EUCLID3)

- LTM : Lycée Technique Moknine (Saïd AbiBakr),

Janvier 1992- Septembre1993

Professeur d'Enseignement Secondaire. 


\section{Principales Activites Pedagogiques}

Ecole Nationale d'Ingénieurs de Monastir :

1998 - Présent

- Cours intégrés : Etude de conception, classe MECA2

- Cours intégrés : Conception Assistée par Ordinateur, classe MECA1 ;

- Cours intégrés : Conception Intégrée, classe MECA3

- Cours intégrés : Fabrication Assistée par Ordinateur, classe MECA3 ;

- Cours intégrés : Modélisation Géométrique et CAO, classe MECA3 ;

- Cours : Méthodes et modèles de conception de systèmes mécaniques, MASTERE GM (03-06)

- TP : Etude de projets de conception, classe MECA2 ;

- TP : CAO, logiciels Pro/Engineer et CATIA, classe MECA1 ;

- TP : Calcul par éléments finis, logiciel ANSYS, classe MECA2 ;

- TP : FAO , logiciel CATIA, MECA3

- Encadrement de projets de fin d'études d'ingénieurs.

Ecole Polytechnique de Tunisie :

2001 - Présent

- Cours: Conceptique, classe $3^{\text {èmme }}$ année, option MEST

- Encadrement de projets de fin d'études d'ingénieurs.

\section{ACtivites AdMinistratives :}

- Directeur du Département de Génie Mécanique, ENIM, Monastir ;

$1999-2002$

- Membre élu du Conseil Scientifique de l'ENI de Monastir ;

- Membre du Jury National de Recrutement des Technologues ;

$2005-2008$

- Membre du Comité de Direction du Laboratoire LGM ;

$2004-2005$

$2004-2005$

\section{Publications SCIENTIFIques : (6 ARTICLES, 18 COMMUNICATIONS)}

\section{Articles de Revues Publiés (6)}

Après la Thèse : 4 (Période 2000 - 2005) :

- B. Louhichi, A. BenAmara, V. François ; «Intégration CAO/Calcul par reconstruction des modèles $\mathrm{CAO}$ à partir des résultats de calcul» Revue Internationale d'Ingénierie Numérique, Volume $1-\mathrm{n}^{\circ} 1 / 2005$. Éditions Hermès - Lavoisier 2005. (cf. fiche page 115)

- A. Chamekh, H. BelHadjSalah, M. A. Gahbiche, A. BenAmara, A. Dogui, "Identification of the hardening curve using a finite element simulation of the bulge test" Steel GRIPS Journal of steel and related materials; Septembre 2004 741-746 (2004). (cf. fiche page 116)

- N. Aifaoui, A. BenAmara, A. Dogui, "Interopérabilité des processus de conception et de calcul »; Journal Européen de systèmes automatisés ; Vol 38-3-4 (2004). (cf. fiche page 117)

- A. BenAmara, N. Aifaoui, D. Deneux, "Intégration CAO/Calcul, une démarche fonctionnelle intégrée" ; Journal Européen des Systèmes Automatisés ; Volume 34, º2-3, (2000). (cf. fiche page 118) 


\section{Avant la Thèse : 2 (Période 1994 - 1998)}

- D. Deneux, A. BenAmara ; "Conception fonctionnelle intégrant le calcul mécanique". Revue Internationale de CFAO et d'informatique graphique, Éditions Hermès, Vol. 13, n², pp 191204, juin 1998. (cf. fiche page 119)

- A. BenAmara, D. Deneux; R. Soenen, A. Dogui "Pré dimensionnement en conception fonctionnelle". Revue Internationale de CFAO et d'Informatique graphique, Éditions Hermès, Vol. 11, $\mathrm{n}^{\circ} 1-2$, ISSN: 0298-0924, pp. 133-147, février 1996. (cf. fiche page 120)

\section{Articles de Revues Soumis : 2 ( 2005 )}

- A. BenAmara, V. François, B. Louhichi; "CAD/Analysis integration: Building CAD models using FEA deformed mesh" Revue CAD, soumis mai 2005.

- A. BenAmara, N. Aifaoui, B. Louhichi, V. François, D. Deneux; « Numerical integration and interoperability of CAD and analysis processes"; Revue Mécanique et Industrie ELSIVIER ; soumis avril 2005.

\section{Congrès et Conférences Nationales et Internationales (18)}

Après la Thèse : 15 (Période 2002 - 2005) :

- V. François, B. Louhichi, A. BenAmara ; «Reconstruction d'un modèle CAO à partir d'un modèle éléments finis déformé », XVII Congrès Français de Mécanique, UTT, Troyes, France, 29 Août - 02 septembre 2005. (cf. fiche page 122)

- B. Louhichi, N. Aifaoui, A. BenAmara, V. François, D. Deneux ; «Intégration numérique et interopérabilité des processus de CAO et de Calcul », XVII Congrès Français de Mécanique ; UTT -Troyes, France, 29 Août - 02 septembre 2005. (cf. fiche page 123)

- B. Louhichi, A. BenAmara, V. François, L. Romdhane ; «Rebuilding of CAD Model Starting from the Finite Elements Results» Le 20e Congrès Canadien de Mécanique Appliquée: CANCAM 2005 ; Université de McGill; Canada, 30 mai au 2 juin 2005. (cf. fiche page 124)

- B. Louhichi, V. François, A. BenAmara ; «Intégration de la méthode des éléments finis dans le processus de $\mathrm{CAO}$ : détermination de la géométrie d'un modèle déformé » $73^{\mathrm{e}}$ Congrès de l'ACFAS, Université du Québec à Chicoutimi, Canada, 9 - 13 mai 2005. (cf. fiche page 125)

- B. Louhichi, A. BenAmara, V. François ; «Reconstruction d'un modèle BREP déformé à partir des résultats d'une simulation éléments finis » Premier Congrès International Conception et Modélisation des Systèmes Mécaniques: CMSM2005; 23-25 Mars 2005; Hammamet - Tunisie. (cf. fiche page 126)

- I. Hajjaji, A. BenAmara, R. Fredj «Etude Expérimentale de l'influence des paramètres de coupe sur l'état de surface en tournage dur» Premier Congrès International Conception et Modélisation des Systèmes Mécaniques: (Poster) CMSM2005; 23-25 Mars 2005 ; Hammamet - Tunisie. (cf. fiche page 127)

- R. Fredj, A. BenAmara; "Aperçu des techniques de prédiction de défauts des surfaces usinées " Premier Congrès International Conception et Modélisation des Systèmes Mécaniques : CMSM2005; 23-25 Mars 2005 ; Hammamet - Tunisie. (cf. fiche page 128)

- N. Aifaoui, A. BenAmara; «Les Features de calcul : Un modèle générique d'intégration $\mathrm{CAO} / \mathrm{Calcul»}$ Premier Congrès International Conception et Modélisation des Systèmes Mécaniques : CMSM2005; 23-25 Mars 2005 ; Hammamet - Tunisie. (cf. fiche page 129) 
- B. Louhichi, A. BenAmara, V. François ; «Simulation cinématique d'un assemblage dans les conditions de fonctionnement» $72^{\mathrm{e}}$ Congrès de l'ACFAS, Université du Québec à Montréal, Canada, 10 - 14 mai 2004. (cf. fiche page 130)

- W. Guenenna, A. BenAmara, F. Sakli, «Optimisation des plans de matelassage à l'aide des algorithmes génétiques » The First International Conference of Applied Research in Textile ; CIRAT ; pages 143-150, Monastir (2004). (cf. fiche page 131)

- N.Aifaoui, A. BenAmara, D. Deneux " Towards a management model of design and mechanical analysis processes based on feature concept"; $5^{\text {ème }}$ conférence internationale sur la conception et la fabrication intégrées en mécanique IDMME 04, Bath, UK avril 2004. (cf. fiche page 132)

- A. Chamekh, M. A. Gahbiche, H. Bel Hadj Salah, A. BenAmara \& A. Dogui, “ Etude expérimentale et numérique de l'essai de gonflement hydraulique", Actes des JS2003, T2, pp 222-227, 21-22 mai (2003), Borj Amri, Tunisie. (cf. fiche page 133)

- N. Ifaoui, D. Deneux, A. BenAmara, R. Soenen, A. Dogui, "Mechanical analysis process modelling based on analysis features", IEE international conference SMC' Hammamet, Tunisie, (6-9 oct 2002). (cf. fiche page 134)

- F. Bouabda, A. BenAmara, "Modèle de conception basé sur les entités technologiques: Application à la conception des moules d'injection plastiques“, CRATT' 2002 ISET RADES, Tunisie (2002). (cf. fiche page 135)

- N. Ifaoui, D. Deneux, A. BenAmara, R. Soenen, A. Dogui, "CAD/ Analysis integration based on generic model of mechanical analysis process", $4{ }^{\text {ème }}$ conference internationale sur la conception et la fabrication intégrées en mécanique: IDMME'02, IFMA, CLERMONTFERRAN, France, (14-16-mai 2002). (cf. fiche page 136)

Avant la Thèse : 3 (Période 1996 - 1998) :

- D. Deneux, A. BenAmara; «Intégration du calcul mécanique dans un processus de conception fonctionnelle », Lettre de l'Association Française MICADO, ISSN : 1261-5137; pages 2-3 ; Septembre 1998.

- A. BenAmara, D.Deneux ; "F-CAD : un prototype de système de CAO fonctionnelle pour l'intégration du calcul dans le processus de conception", ". $17^{\text {zème }}$ conférence internationale MICAD: MICAD'98, Paris, 19 mars 1998.

- A. BenAmara, D. Deneux, R.Soenen, A.Dogui; "CAD / Analysis integration - An object oriented approach". $1^{\text {ère }}$ Conférence Internationale sur la Conception et Fabrication intégrées en mécanique (IDMME'96), Nantes, 1996., pp. 613-622.

\section{Projets de Collaboration Scientifique (2)}

Projet DGRST-CNRS : 2003

(cf. fiche page 154 - 156)

- Responsables : A. BenAmara, LGM-ENIM / D. Deneux, LAMIH-UVHC France

- Thème : Modélisation de vêtements par features d'assemblages intégrant les lois de comportement des matériaux souples : Contribution à la confection assistée par ordinateur.

- Etudiants Impliqués : Adel Amri (Mastère soutenu), Wisem Guenanna (Thèse en cours)

\section{Projet DGRST-CNRS : 2001}

(cf. fiche page 157 - 158)

- Responsables : A. BenAmara, LGM-ENIM / R. Soenen, LAMIH-UVHC France

- Thème : Modèles Innovants en Conception Mécanique.

- Etudiants impliqués : Nizar Aifaoui (Thèse soutenu en co-tutelle, juillet 2003). 


\section{ENCADREMENTS SCIENTIFIQUES}

Thèses de Doctorat : (1 soutenue, 3 en cours)

- Thèse en cotutelle, ENIM / UVHC - France. (cf. fiche page 139 - 142)

Soutenue 03/07/2003

Titre : Intégration CAO/Calcul : Une approches par les features de calcul

Co-encadreurs : Prof. A. Dogui, Prof. René Soenen, Prof D. Deneux

Doctorant : Nizar Ifaoui

- Thèse en co-direction, ENIM / UQTR - Canada. (cf. fiche page 143 - 144)

Sept. 03 - présent

Titre: Contribution à l'intégration $\mathrm{CAO} / \mathrm{Calcul}$ par reconstruction des modèles $\mathrm{CAO}$ à partir des résultats d'une analyse élément finis.

Co-encadreurs : $\mathrm{M}^{\mathrm{r} .}$ V. François, Prof. UQTR et $\mathrm{M}^{\mathrm{r} .}$ L. Romdhane, M.C. ENIM

Doctorant : Borhène Louhichi

- Thèse en co-direction, ENIM. (cf. fiche page 145)

Sept. 03 - présent

Titre : Caractérisation mécanique de l'usinage en finition

Co-encadreur : $\mathrm{M}^{\mathrm{r} .}$ L. Romdhane, M.C. ENIM

Doctorant : Ramzi Fredj

- Thèse en codirection, ENIM. (cf. fiche page 146)

Sept. 03 - présent

Titre : Contribution à l'élaboration d'algorithmes d'optimisation des problèmes de matelassage et de placement, application à l'industrie de la confection textile.

Co-encadreur : $\mathrm{M}^{\mathrm{r}}$ F. Sakli, Prof. ISET Ksar Helal.

Doctorant : Wisem Guenanna.

Mastères de Génie Mécanique : (5 soutenus)

- Mastère de génie mécanique ENIM, (cf. fiche page 148)

Soutenu $25 / 05 / 2005$

Titre: Développement d'un solveur élastoplastique au tour d'un mailleur tridimensionnel optimisé.

Co-encadreur : H. BenlHadjSalah, M.C. ENIM.

Etudiant : Khaled Souaissa

- Mastère de génie mécanique ENIM, (cf. fiche page 149)

Soutenu 13/05/ 2005

Titre : Etude expérimentale et numérique de tournage dur

Etudiant : Imed Hajjaji

- Mastère de génie mécanique ENIM, (cf. fiche page 150)

Soutenu 05/04/2003

Titre : Simulation numérique de l'emboutissage des tôles minces

Co-encadreur : H. BenlHadjSalah, M.C. ENIM.

Etudiant : Abdessalem chamekh

- Mastère de génie mécanique ENIM, (cf. fiche page 151)

Soutenu 07/07/2003

Titre : Reconstruction d'un modèle BREP à partir des résultats d'une analyse E. F.

Co-encadreur : Mr. V. François, Prof. UQTR, Canada.

Etudiant : Borhène Louhichi

- Mastère de génie mécanique ENIM, (cf. fiche page 152)

Soutenu 02/03/2002

Titre : Modèle de conception basé sur les entités technologiques.

Etudiant : Fethi Bouabda 


\section{ENCADREMENTS DE PROJETS INDUSTRIELS.}

\section{Références Industrielles à l'Etranger (5):}

- ENI-METZ, France / STIA, Sousse Tunisie

Septembre - Janvier 2004/2005:

Calcul de dimensionnement et optimisation d'un châssis de BUS.

- DGM, Université Laval, Canada :

Février - Juin 2005 Interprétation d'un modèle CAO pour la programmation d'un algorithme de désassemblage.

- DGM, Université du Québec à Trois Rivières, Canada :

Février - Juin 2003

Développement d'un solveur élastique éléments finis à l'aval du mailleur tridimensionnel intégré à la CAO.

- SNECMA-LABINAL, Toulouse, France :

Février - Juin 2002/2003

Six projets distincts : Conception de composants pour avion airbus.

- CER METZ, France :

Février - Juin 2001

Etude de la qualité des pièces forgées.

\section{Références Industrielles en Tunisie : (8)}

- SOGERMA - Tunisie, Monastir :

Février - Juin 2005

Mise en place d'un système d'aide à la maintenance des structures d'avion Airbus A320.

- CPG : Gafsa

Février - Juin 2003:

Etude de conception et réalisation d'une machine d'essai de flexible pour engins de chantier.

- CLIMATHERM, Tunis :

Février - Juin 2001

Préparation à la mise en place d'un système d'assurance qualité ISO 9002.

- STIP, Msaken :

Février - Juin 2002

Etude de conception et réalisation d'une machine d'enroulement du tissu de service sur la tourelle des machines de confection A70.

- PULSAR Tech, Tunis

Février - Juin 2001:

Développement d'un mini système de $\mathrm{CAO}$ volumique.

- BUTINORD, Medjaz Elbab :

Etude de conception et réalisation d'une machine de gaufrage de papier aluminium sur des bandes bitumineuses.

- CSPS, Sousse :

Conception et fabrication intégrées d'une pièce en plastique.

\section{CONVENTIONS DE COLLABORATION SCIENTIFIQUE (3)}

- Université de Valenciennes et du Hainaut Cambrésis, France :

Echange d'étudiants et stagiaires,

Conclusion des conventions de co-tutelle de thèse,

- Université du Québec à Trois Rivières, Canada :

Echange d'étudiants et stagiaires,

Conclusion des conventions de co-tutelle de thèse,

- Ecole Nationale d'Ingénieurs de Metz, France :

Présélection des candidats tunisiens pour le concours étranger d'accès au groupe ENI en France. Niveau Bac, ISET et Classes Préparatoires, Maîtrise),

Préparation d'un projet de co-diplômation (ENI-Monastir, ENI-Metz), 


\section{PARTICIPATION A DES MANIFESTATIONS SCIENTIFIQUES NATIONALES (7) ET INTERNATIONALES (13).}

Membre du comité scientifique : (2)

- PENTOM, Valenciennes, France, 2003 ;

- FEATS, Valenciennes, France, 2001;

Membre du comité d'organisation : (5)

- CMSM'05, Membre du comité d'organisation

Hammamet, Tunisie, Mars 2005.

- Journée de l'Ingénierie 2002, JI'2002

Monastir, Tunisie, Mars, 2002

- Journée de l'Ingénierie 2004, JI'2004

- Journée d'Actualisation des Connaissances en CFAO,

Sousse, Tunisie, Décembre, 2004

- Journée d'Actualisation des Connaissances en UGV,

Monastir, Tunisie, Février, 2005

- CMSM'07, Président du comité d'organisation

Monastir, Tunisie, Décembre, 2005

Sousse, Tunisie, Mars 2007.

Conférencier / Coauteur : (14)

- Congrès Français de Mécanique, CFM’05

Troyes, France, 29/08 au 02/09 2005

- CANCAM 2005,

- 73e congrès de l'ACFAS,

- CMSM'05, Hammamet, Mc Gill, Montréal, Canada, Juin 2005 Chicoutimi, Canada, 09 - 13 Mai 2005 Tunisie, Mars 2005

- 72e congrès de l'ACFAS,,

- CIRAT, Monastir,

- IDMME 05,

- J.S. des académies militaires,

- CRATT'02,

- IDMME'02,

- IEEE, SMC02,

- CPI'99,

- MICAD’98,

Paris, France, 19 Mars 1998

- IDMME'96,

ECN, Nantes, France, Avril 1996

\section{CONNAISSANCES INFORMATIQUES}

- Bureautique : Word, Excel, Power Point, Access, ...

- Système: Windows, Mac OS et Unix.

- Programmation : $C, C++$, développement Web, ...

- Logiciels de CFAO : CATIA, SolidWorks, Pro/Engineer, TopSolid,...

- Logiciels de Calcul par Eléments Finis : ANSYS, COSMOS, DESIGN START,

\section{LANGUES}

- Arabe, Français et Anglais. 


\section{Introduction Générale}

Ce mémoire présente une synthèse de mes principales activités de recherche qui jalonnent mon parcours d'enseignant - chercheur depuis ma thèse de doctorat, soutenue en décembre 1998 en cotutelle entre l'Université de Valenciennes en France et l'Ecole Nationale d'Ingénieurs de Tunis en Tunisie, jusqu'à la fin de l'année universitaire 2004/2005.

Mes activités de recherche sont menées au Laboratoire de Génie Mécanique de l'Ecole Nationale d'Ingénieurs de Monastir et en collaboration avec le Laboratoire d'Automatique et de Mécanique Industrielles et Humaines de l'Université de Valenciennes en France et le Département de Génie Mécanique de l'Université du Québec à Trois Rivières au Canada.

Ces travaux s'inscrivent dans le cadre général de la tendance d'intégration des applications de $\mathrm{CAO}, \mathrm{FAO}$ et Calcul et s'orientent plus particulièrement vers l'intégration $\mathrm{CAO} / \mathrm{Calcul}$. En effet, depuis plus de quinze ans, cette thématique a fait l'objet de mes principales activités de recherche. J'ai débuté dans ce domaine par un stage de DEA, qui a porté sur la réalisation d'une interface entre le logiciel Pro/Engineer et le code de calcul par éléments finis MODULEF, et j'ai ensuite enchaîné par une thèse, sur l'intégration de la composante calcul dans une démarche de conception fonctionnelle intégrée. Cela m'a permis ensuite d'orienter principalement mes recherches (encadrements et collaborations scientifiques) vers cet axe de recherche d'actualité.

De notre point de vue, les applications de CAO et de Calcul doivent plus que jamais s'édifier sur des modèles compatibles et réactifs en vue de supporter la complexité croissante des produits et des processus de conception. L'évolution de l'environnement de conception est actuellement marquée par une participation de plus en plus forte d'acteurs dont les compétences sont variées (plusieurs outils) et souvent répartis sur des sites géographiquement éloignés (entreprise étendue). Ces objectifs induisent des problèmes de modélisation des données et des processus spécifiques aux applications de CAO et de Calcul.

Nos travaux de recherche s'inscrivent exactement dans ce cadre et consistent à proposer et valider des modèles permettant de rehausser sensiblement le niveau d'intégration entre les applications de $\mathrm{CAO}$ et de Calcul en vue d'attendre une interopérabilité compatible avec les exigences actuelles.

Ce mémoire présente une synthèse de nos travaux. Il est structuré en cinq parties :

La première partie présente une introduction et une mise en situation de la thématique « intégration $\mathrm{CAO} / \mathrm{Calcul} »$. Elle permettra aux lecteurs de voir de plus près les objectifs et les problèmes liés à cette thématique.

La deuxième partie présente un état de l'art dans le domaine de l'intégration $\mathrm{CAO} / \mathrm{Calcul}$ permettant d'avoir une référence pour mieux positionner nos travaux par rapport à la communauté nationale et internationale.

La troisième partie sera consacrée à la présentation d'une synthèse de nos principaux résultats de recherche en matière d'intégration $\mathrm{CAO} / \mathrm{Calcul}$. Nous ferons le point sur les différents travaux entrepris dans ce domaine et nous détaillerons les approches et les modèles proposés. 
La quatrième articulation de ce mémoire est indépendante des autres parties. Nous y présentons une synthèse de nos travaux de recherche en émergence qui constituent une ouverture sur d'autres champs de la recherche en génie mécanique.

Nos conclusions et perspectives à moyen et long terme font l'objet de la cinquième partie de ce mémoire. 


\section{PARTIE I :}

Introduction et mise en situation 


\section{PARTIE I}

\section{Introduction et mise en situation}

\section{Introduction}

De nos jours, nous assistons à un développement du travail en mode projet qui se caractérise par la mobilisation de compétences multiples. La globalisation des marchés ainsi que la réduction des coûts et des délais de développement de nouveaux produits s'est traduit, au niveau des outils supports de la conception calcul et fabrication, par des nouvelles exigences en terme de compatibilité et de réactivité en vue de s'adapter au contexte de travail collaboratif, voire distribué, tout en assurant la structuration, le suivi, et la traçabilité des échanges. Cela a induit un accroissement considérable des besoins en terme de communication inter applications et de cohérence globale des systèmes supports des différents modèles du produit (CAO, Calcul, FAO).

De par leur forte interdépendance, les deux activités CAO et calcul seront donc amenées à s'édifier sur les nouvelles technologies émergentes dans les domaines de la modélisation des données (modèle de produit) et des processus (interopérabilité) afin de pouvoir prendre en compte la manipulation d'objets hétérogènes (géométrie, sollicitations, maillage, déformation, etc.). En effet, l'intégration de ces applications est devenue une des principales préoccupations en CFAO. L'objectif recherché est d'aller plus loin que les échanges statiques basés sur les standards (iges, set, dxf, etc.) et de favoriser l'échange et le partage des données sans recopies ou transformations manuelles afin de fluidifier les flux d'informations entre $\mathrm{CAO}$ et Calcul tout en garantissant la fiabilité et la traçabilité des données.

Tout au long du cycle d'élaboration d'un produit, l'activité de conception est envisagée comme un ensemble de tâches interdépendantes (tâches de modélisation géométrique, tâches de calcul, tâches de fabrication, tâches d'assemblage, etc.) caractérisées par des spécifications, des contraintes, et un ensemble de solutions candidates plus ou moins pertinentes. Pour solutionner ce problème, sous contraintes et à choix multiples, le concepteur engage sa responsabilité en effectuant des choix grâce à des connaissances représentatives de son métier. Une partie de ces connaissances peut être mémorisée par le système support de la conception (modèles géométriques paramétrés, indexation de cas types de conception, procédures de calcul systématique, ...).

Cependant, dans un contexte de conception intégrée, les choix (arbitraires ou non) d'un acteur de la conception deviennent, pour d'autres acteurs, soit des objectifs à atteindre, soit des contraintes sur leurs activités. Tout ceci converge vers le besoin d'une méthodologie qui conduit non seulement à la génération d'un modèle produit qui comporte l'ensemble des concepts permettant de décrire de manière complète et générique les différents aspects du produit, mais aussi à l'édition d'un modèle de processus de conception favorisant un aspect dynamique et coordonné des tâches de conception. Cela permettra une remise en cause plus rapide des spécifications et d'envisager le traitement en parallèle, de tâches appartenant à des phases de conception différentes.

Le calcul mécanique est une des tâches les plus importantes liées à l'activité de conception. Elle est répartie tout au long du cycle d'élaboration du produit et permet de prédire son comportement mécanique [Lalane 87] [Gautier 96]. En conception mécanique, deux types de calcul peuvent être envisagés : un calcul de dimensionnement, qui consiste à déterminer les dimensions en fonction des sollicitations; et un calcul de validation, qui consiste à vérifier si les composants du produit résistent aux contraintes appliquées.

Aujourd'hui, plus que jamais, les systèmes supports de l'activité de conception doivent permettre d'intégrer le calcul mécanique au cours de la conception. Cette intégration doit être prise au sens large du terme, c'est-à-dire les acteurs peuvent aller plus facilement de la $\mathrm{CAO}$ vers le calcul et inversement. 
Cela permettra de minimiser les classiques allers-retours entre conception et calcul et réduit largement le coût de la conception.

\section{Qu'est ce que l'intégration?}

En CFAO, l'intégration consiste à associer en un tout cohérent des applications hétérogènes $(\mathrm{CAO}$, Calcul, FAO, etc.), avec l'objectif de favoriser au maximum l'action simultanée ou concourante des ces applications au cours du processus global et de minimiser la traduction de données qui constituent le facteur principal de perte de temps et de sémantique.

\section{L'intégration CAO/Calcul.}

En ce qui concerne les tâches conception et calcul, nous distinguons essentiellement deux types d'outils de modélisation: Les modeleurs CAO et les outils de calculs (solveurs). Les modeleurs CAO permettent de définir la morphologie du produit et sont basés sur des architectures dédiées à la modélisation géométrique. En revanche, les solveurs manipulent des entités de natures différentes (poutre, maillage, efforts, etc.). En général, la description géométrique des pièces modélisées en CAO est transférée vers des solveurs, via des interfaces, pour analyser et évaluer leurs comportements.

L'étude de l'activité calcul dans le processus de conception mécanique [BenAmara 98], montre que la fonction calcul intervient non seulement dans les phases de spécification des solutions technologiques et techniques, mais aussi lors de la détermination des différents paramètres et l'évaluation de ces solutions. La composante calcul intervient donc dans presque toutes les étapes du processus de conception mécanique.

De ce fait, nous considérons que l'activité calcul est un ensemble de tâches réparties dans tout le processus de conception, et qu'une réelle intégration de ces tâches implique la satisfaction des besoins suivants :

- Le modèle de produit doit supporter non seulement les informations géométriques (géométrie, contraintes), mais aussi des informations technologiques relevant du domaine de calcul (conditions aux limites et matériaux).

- Le modèle de processus de conception doit supporter le déroulement de l'ensemble des tâches de conception. Ce modèle doit permettre au concepteur de faire appel aux tâches de calcul aux différents niveaux de modélisation du produit.

- Le système support de la modélisation du produit doit pouvoir communiquer avec les outils d'analyse et d'aide à la conception pour permettre au concepteur de spécifier, dimensionner et évaluer les différentes solutions proposées. D'où la nécessité d'outils efficaces d'échange de données.

Ces remarques nous amènent à constater que les principaux facteurs de l'intégration $\mathrm{CAO} / \mathrm{Calcul}$ sont : le modèle de produit, le modèle de processus de conception et le modèle d'échange de données.

\subsection{Modèle de Produit}

Le modèle de produit est une abstraction du produit réel. Il doit permettre, d'une part, la structuration des relations entre les différents objets instanciés au cours du processus de conception (modèle conceptuel), et d'autre part, la description fonctionnelle, géométrique, topologique et technologique du produit (modèle descriptif). Ce modèle comporte également des informations relatives au comportement des différents composants du produit (modèle de comportement). Nous décrivons ci-dessous ces trois types de modèles.

- Le modèle conceptuel: Ce modèle est constitué par l'ensemble des concepts qui décrivent le produit aux différents niveaux de modélisation: fonctionnel, technologique, et technique. 
- Le modèle descriptif : Ce modèle permet la description du produit en fonction de l'étape de modélisation. Par exemple, dans la phase de modélisation géométrique le modèle descriptif est une représentation idéalisée du produit réel. En effet, il s'agit de présenter les composants mécaniques en modélisant leur géométrie par des surfaces parfaite (pas de défaut géométrique). Cependant, la description du produit lors d'une analyse par éléments finis est assurée par des entités de type : nœud, élément, contrainte, etc.

- Le modèle de comportement : Il s'agit des modèles formels [Papegay 92] permettant de décrire non seulement le comportement global du produit, mais aussi le comportement propre à chacun de ses constituants (éléments standards, pièces élémentaires, ... point matériel). Ces modèles sont utiles pour simuler le fonctionnement du produit physique et pour effectuer les calculs des mouvements, des efforts, de transfert d'énergie, etc. Nous citons à titre d'exemple, les modèles de comportement des éléments standards (moteur, vérin, roulement), les lois de comportement des matériaux (relation effort déformation : loi de Hooke).

En ce qui concerne les phases $\mathrm{CAO}$ et Calcul, on distingue plus particulièrement les différentes modélisations suivantes.

\subsubsection{Modèle de CAO paramétrique}

La conception paramétrique consiste à utiliser un modèle CAO basé sur des paramètres essentiellement géométriques et des éventuelles relations entre ces paramètres. Cela permet de gérer de façon plus simple les modifications introduites au niveau des différents paramètres des pièces et assemblages mécaniques. Les relations sont définies sous formes d'équations mathématiques ce qui permet d'exprimer des éventuelles relations complexes entres les différents paramètres du produit.

\subsubsection{Modèle de CAO variationnel}

Par opposition à la conception paramétrique, l'approche paramétrique permet de répondre à une plus large variété de problème de conception. Basée sur un solveur de contraintes géométriques ou dimensionnelles, elle permet d'adapter les solutions de conception en fonction de la variations de différents paramètres de conception tout en gardant valables les différentes contraintes.

Exemple du projet MRT

\subsubsection{Modèle de CAO basé sur les Features}

Le concept de features a été initialement élaboré pour résoudre des problèmes d'intégration entre la conception et la fabrication. Actuellement, il est utilisé pour résoudre d'autres types de problèmes de conception. De ce fait, plusieurs définitions ont été attribuées à ce concept. On constate que ces définitions sont relatives aux deux points de vue suivants : un premier point de vue qui relève de la fabrication et un second qui relève de la conception [Salomons 95a]. Nous donnons ci-dessous quelques définitions relatives à ce concept.

- Forme géométrique ou entité dont les caractéristiques sont utiles pour assurer une fonction de fabrication ou d'autres tâches de conception [Luby \& Dixon 86].

- Partie distinctive ou caractéristique d'une pièce, définissant une forme géométrique spécifique à un processus de fabrication ou pouvant être utilisée dans un processus d'assemblage ou de contrôle [Erve 88].

- Groupement sémantique utilisé pour décrire une pièce et son assemblage [Giacometti 91].

- Ensemble d'informations relatif à la description d'une pièce [Shah 88]. Shah classifie les features de la manière suivante: features de forme, features de précision, features technologiques et features administratives, [Shah 89].

- Forme générique ayant un sens ingénieriste [Wingard 91]. 
- Ensemble d'informations (géométriques ou autres) référant un aspect particulier d'une pièce [Salomons 93], [Salomons 95b], [Loriod 89].

Nous constatons des différences de point de vue dans les définitions présentées ci-dessus. Une première famille de définitions centrées sur l'aspect forme ; justifiée par le fait que l'utilisation du concept de feature reste plus liée au domaine de l'intégration de la conception et de la fabrication [Erve 88], [Luby \& Dixon 86], [Requicha 86]. Dans une seconde famille de définitions, on constate l'évolution du concept de feature vers des significations non forcement géométriques. Nous distinguons en particulier les travaux suivants : [Femex 96], [FEAST 94], [Daugy 94], [Shah 90], [Laako 90], [Anderson 90], [Roller 89], [Pratt 89], [Gossard 88].

\subsubsection{Modèle de Calcul}

En ce qui concerne le modèle mécanique utilisé pour le calcul, il est basé sur des hypothèses simplificatrices portant non seulement sur la géométrie, mais aussi sur les conditions aux limites (sollicitations et liaisons) et sur le comportement mécanique (élastique, élastoplastique, etc.).

Les applications de calcul mécanique sont dans la majorité des cas basées sur la méthode des éléments finis. Le modèle mécanique utilisé comporte une discrétisation de la géométrie réelle (maillage) et une descriptions des conditions aux limites (les efforts, températures, liaisons, etc.) et du comportement du matériau (élastique, élastoplastique, etc.).

La construction de ce modèle mécanique servant pour le calcul est souvent couplée à la phase de $\mathrm{CAO}$ notamment en ce qui concerne la phase de maillage qui consiste à une discrétisation (décomposition en élément simple) du modèle CAO. Dans le cas des mailleurs intégrés à la CAO cette phase, les conditions aux limites et la définition du matériaux sont effectués du coté du système de CAO.

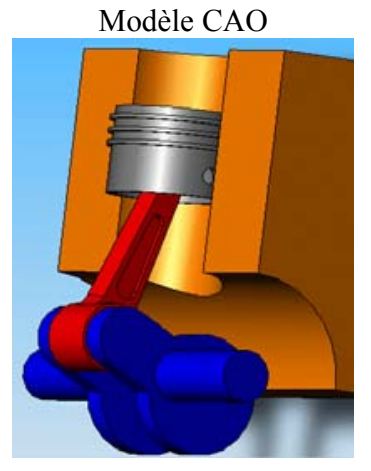

\section{De la CAO vers le Calcul}

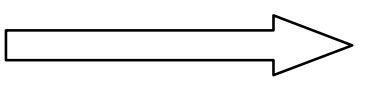

Figure 1 : Passage de la CAO vers le Calcul.

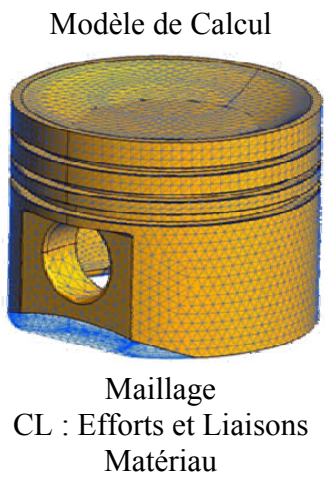

\subsection{Processus CAO et Calcul}

De façon générale, au cours du processus de conception on commence par spécifier des solutions mécaniques (spécification fonctionnelle et géométrique), puis on détermine les paramètres qui les caractérisent (forme, dimensions, matériaux, sollicitations, etc.) et enfin on en évalue les performances par rapport à l'ensemble des contraintes (dimensionnement et étude du comportement). Conformément au principe d'essai-erreur [Degirmenciyan 95], le calcul mécanique intervient souvent dans presque tous les niveaux de modélisation du produit pour valider ou remettre en cause les solutions proposées. Cela entraîne bien évidemment d'inévitables modifications dans les différents modèles et, par conséquent, plusieurs itérations sont en général nécessaires entre les étapes de modélisation et celles de calcul et d'évaluation des performances avant d'aboutir à une solution acceptable. La recherche d'une solution optimale augmente encore le nombre d'itérations.

Dans un contexte de conception et calcul assistés par ordinateur (CAO et Calcul EF), ces interactions nécessitent plusieurs échanges de données entre les outils de CAO, d'une part, et les outils de calcul d'autre part. 


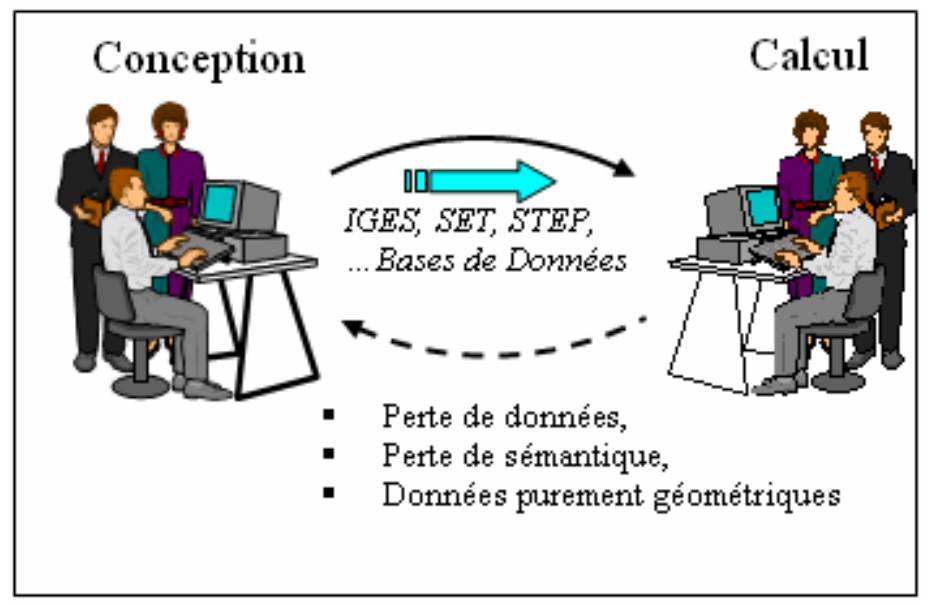

Figure 2 : Echange de données CAO - Calcul.

Par ailleurs, la demande des bureaux d'étude, basée sur un large consensus industriel, s'oriente actuellement vers des systèmes intégrés [Marty 91] capables de supporter l'évolution des modèles et la prise en compte des contraintes qui peuvent intervenir ultérieurement et qui sont les principaux responsables des coûteux aller-retour entre les différents niveaux de modélisation [Féru 94]. Ces nouvelles tendances sont marquées par l'apparition de deux nouvelles approches de l'ingénierie de conception : ingénierie simultanée, ingénierie concourante.

\subsection{Modèle d'échange de données}

L'idée d'automatiser le transfert des données entre les systèmes de CAO et les outils d'analyse a été communément retenue comme solution de base pour surmonter le problème de la double saisie des données. L'utilisation des formats standards d'échanges (IGES, SET, STEP, ...) a évité ce problème de double saisie des données de manière remarquable. En effet, il est actuellement possible de construire automatiquement un maillage à partir d'une modélisation géométrique CAO et de contrôler la densité du maillage [Cuillière 93] [Cuillère 97]. Mais ces interfaces présentent l'inconvénient de transcoder la géométrie telle quelle sans l'adapter au métier traité par l'outil d'analyse [Labat 92]. Le transfert des données ne va pas au-delà d'une simple conversion des données, il est incapable de tenir compte des modifications inévitables imposées par le projet au niveau du modèle CAO.

Plusieurs solutions à ce problème ont été proposées [François 98]. Nous présentons ci-dessous les principales approches en distinguant deux techniques différentes de communication entre outils de modélisation hétérogènes. La première regroupe toutes les solutions basées sur les techniques d'interfaçage des systèmes de modélisation (juxtaposition des outils et traduction des données), tandis que la deuxième concerne les solutions basées sur le partage de données, en utilisant des systèmes de gestion de données techniques [Mawussi 95b].

\subsubsection{Intégration par les interfaces}

[Geffrier 91] propose de réaliser le passage du modèle CAO à une description mathématique idéalisée (format exigé par les outils d'analyse) à l'intérieur même du modeleur CAO. On distingue donc une phase de pré-analyse et une autre de pur transcodage entre le modeleur géométrique et le programme de calcul utilisé. La phase de pré-analyse est assurée par un module "MEF" complètement intégré dans l'environnement CAO (CATIA). Ce module permet de générer les concepts relatifs au monde du calcul (nœud, arête, barre, chargement, blocage, etc.).

[Petiau 95] a caractérisé le calcul et le dimensionnement des pièces mécaniques (dans le domaine de l'aviation) en trois grandes phases: calcul des charges, calcul des contraintes et définition des critères de contraintes admissibles. Pour franchir ces différentes étapes, un lien direct CAO-Calcul est assuré par l'interfaçage du modeleur CAO (CATIA) avec un outil d'analyse par éléments finis (ELFINIS). Ce code de calcul rassemble autour de la même base de données tous les aspects de l'analyse structurale. Parmi les possibilités offertes par cette architecture, il faut souligner les capacités 
et les performances des mailleurs automatiques [Stab 91] ainsi que la facilité de régénérer les différentes étapes des processus CAO et calcul grâce aux techniques du "Batch-interactif" [Petiau 95].

Parmi les modèles d'échange de données inter-outils, nous distinguons le standard STEP (Standard for the Exchange of Product model data, défini par la norme ISO 10303). Il s'agit d'un standard international pour la représentation informatique normalisée des données produit. Ce format d'échange permet de cerner l'ensemble des informations géométriques et technologiques qui caractérisent le cycle de vie des produits. Il est basé sur une représentation neutre des informations caractéristiques des produits (indépendant du système qui la génère). Ce standard peut être utilisé avantageusement pour échanger des bases de données techniques [Ghodous 96].

\subsubsection{Intégration par les données}

[Narayanin 92] propose de développer des outils d'analyse autour d'un modeleur CAO, le tout étant intégré dans un même produit. Dans une première étape, il faut définir un modèle CAO (modèle géométrique de produit) qui servira pour la génération du modèle géométrique de calcul (cf. figure 3). Puis dans une seconde étape, dite de pré-processing, on procède à l'introduction des données du problème pour construire enfin le modèle d'analyse. Un solveur intégré (QUICKSOLVER : figure 3) est alors utilisé sans changer d'environnement.

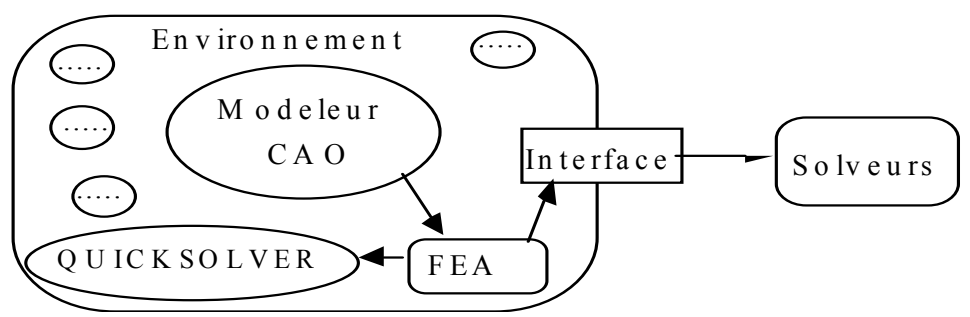

Figure 3 : Environnement QUICKSOLVER .

Ce sous-ensemble de "NASTRAN" (outil d'analyse par éléments finis) ne permet de traiter que les problèmes d'analyse mécanique simple (élasticité linéaire statique, thermique en régime permanent, etc.). Pour les autres cas d'analyse, plus compliqués, on retrouve la solution basée sur l'interfaçage pour transférer le modèle vers d'autres outils de calcul plus performants (NASTRAN, SYSTUS, etc.).

[Labat 92] considère que le dénominateur commun entre les différents métiers ne se limite pas au modèle géométrique. La solution qu'il propose consiste à intégrer les différents métiers dans une même bibliothèque d'éléments paramétrés. Ces éléments possèdent chacun plusieurs représentations adaptées aux métiers visés. Ils intègrent également des paramètres technologiques ou encore des attributs nécessaires à l'analyse. Cette démarche a fait l'objet du logiciel : "PARALEL" qui manipule une bibliothèque d'éléments et utilise le SET paramétré [Labat 92] comme format de stockage de données.

[Saguez 95] défend l'idée d'une intégration $\mathrm{CAO} / \mathrm{Calcul}$ basée sur un partage des données plutôt que sur l'utilisation d'algorithmes de transfert et propose un environnement de conception basé sur la multi-représentation des objets. Pour ce faire, trois tâches principales sont proposées :

- Définition de plusieurs modèles pour un même objet,

- Evolution simultanée des trois modèles,

- Formalisation des relations entre les trois modèles.

Le système proposé est basé sur une approche orientée objet, intégrant un ensemble d'applications $\mathrm{CAO} /$ Analyse (Figure 4), capable de gérer la multi-représentation des objets en conservant l'ouverture vers d'autres systèmes. 


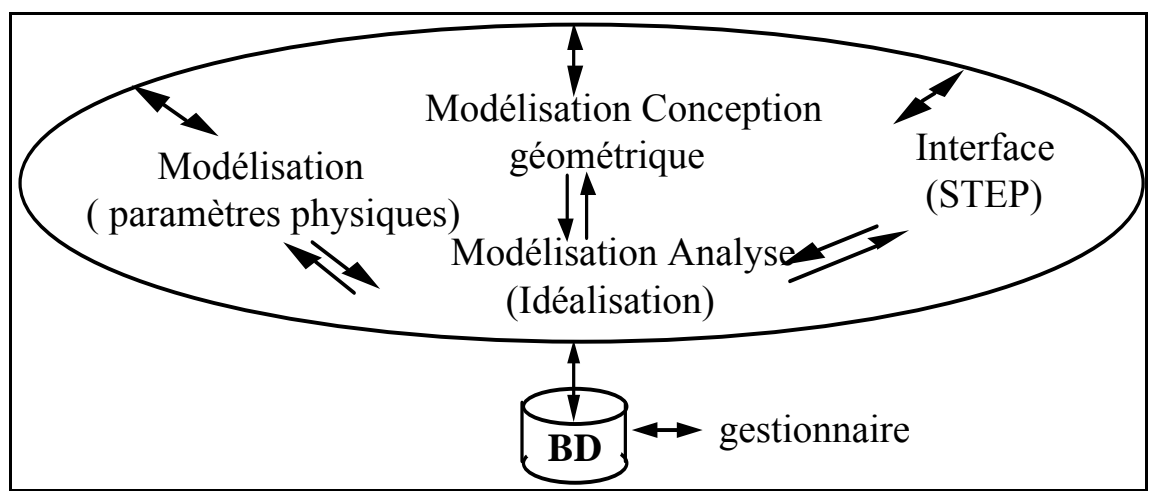

Figure 4 : Environnement d'intégration CAO/Calcul, d'après [SAGUEZ 95].

Dans un tel environnement, les étapes nécessaires pour effectuer les tâches de calcul sont :

- Récupération du modèle CAO,

- Idéalisation du modèle CAO,

- Discrétisation du modèle CAO,

- Entrée des données,

- Calcul et post-processing.

\section{Synthèse.}

Les paragraphes précédents ont permis dans un premier temps de souligner la nécessité de l'intégration des applications de CAO et de Calcul en vue de répondre aux exigences actuelles d'intégration des différentes activités de conception. Dans un second temps, nous avons fait le point sur l'état actuel de solutions proposées pour solutionner ce problème d'intégration.

La première partie de ce chapitre à permit de montre que les principaux facteurs de l'intégration $\mathrm{CAO} / \mathrm{Calcul}$ sont : le modèle de produit, le modèle de processus de conception et le modèle d'échange de données. Ces trois composantes ont ensuite fait l'objet d'une étude bibliographique en vue de faire le point sur les différentes propositions de recherche.

Cette étude nous a permit de présenter l'axe de recherche « intégration CAO-Calcul » par rapport à l'ensemble des travaux de recherche dans le domaine de la CFAO.

Cet axe de recherche a fait l'objet de plusieurs travaux de recherche qui ont conduit à des propositions diverses et variées. Nous consacrons la troisième partie de ce mémoire à l'étude des ces différentes propositions en vue de positionner nos travaux par rapport à la communautés de recherche internationale ayant travaillé sur cet axe de recherche. 


\section{PARTIE II :}

Etat de l'art \& Positionnement des Travaux 


\section{PARTIE II}

\section{Etat de l'art et positionnement des Travaux.}

\section{Introduction}

Imposé par le contexte actuel de la concurrence industrielle, notre sujet de recherche a motivé plusieurs laboratoires de recherche à l'échelle internationale (France, Allemagne, Angleterre, Japon, USA...). Parmi ces laboratoires, nous citons: GILCO, LAMIH, L3S, LGM, LAG, IMAG-LMC, DGM-UQTR au Canada, Imperial College à Londres UK, etc. Ces différents laboratoires collaborent non seulement entre eux, mais aussi avec des industriels sur des projets tels que les projets SALOME, ICARE, OSCAR, etc. L'objectif est une perpétuelle amélioration de l'état d'intégration et d'interopérabilité des différentes tâches de la conception, en l'occurrence la CAO et le calcul mécanique.

Dans ce qui suit, nous allons tracer l'état de l'art dans le domaine de l'intégration $\mathrm{CAO} / \mathrm{Calcul}$ selon deux visions différentes. Une vision qui relève de la collaboration entre laboratoires de recherche et industriels, dans laquelle nous recensons quelques principaux projets de recherche. La deuxième vision relève de la classification des travaux existants que se soit dans le cadre de projets industriels, de thèses de doctorats ou des travaux impliquant les deux aspects. Chose qui favorise de une synthèse qui permettra non seulement de montrer l'efficacité de collaborer avec des industriels pour améliorer l'existant dans le domaine de l'intégration $\mathrm{CAO} / \mathrm{Calcul}$, mais aussi de positionner nos travaux par rapport à la communauté internationale.

\section{Principaux projets impliquant des laboratoires de recherche et des industriels}

\subsection{Le Projet SALOME}

Le projet SALOME [S1] (Simulation numérique par Architecture Logicielle en Open source et à Méthodologie d'Evolution), a pour objectif de définir une plateforme générique d'intégration CAOCALCUL à base de composants réutilisables. Il a été développé sous l'environnement open source OpenCascade [S10] (Figure 5).

Ce projet associe des Laboratoires de recherche, et des éditeurs ou sociétés de services en informatique ainsi que des utilisateurs. Les partenaires sont : MATRA DATAVISION, CEA/DMT, LIP 6, Aérospatiale Matra CCR, INPG/LEG, PRINCIPIA Services, CEDRAT, Bureau Véritas et EDF.

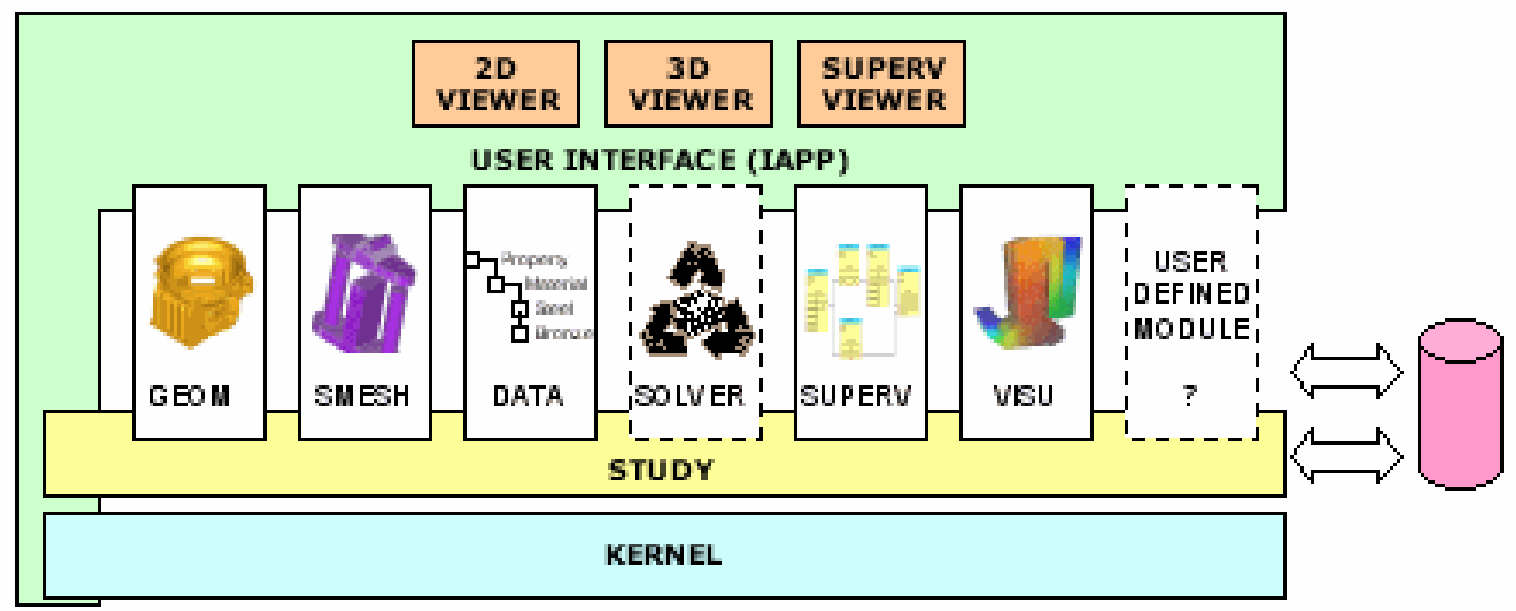

Figure 5 : Architecture du projet SALOME. [S1] 
SALOME est une plate forme de simulation numérique proposant une interface utilisateur générique, conviviale et efficace. L'objectif de projet est la réduction des coûts et délais de réalisation des études et ceci par un couplage facile entre différents logiciels de calcul. Il favorise également la réutilisation de portions de codes et supporte des formats d'échanges entre codes de simulation et logiciels de CAO.

Dans le cadre de l'évolution de la première version de SALOME, Le projet SALOME II a été lancé par le Réseau National des Technologies Logicielles en France. Parmi les Laboratoires membre de ce projet, on trouve le laboratoire $3 \mathrm{~S}$ qui s'est chargé des aspects maillages.

Conçu à la base des composants Open Source d'Open CASCADE, la plate-forme SALOME II compte proposer la base de développement pour les applications de pré et post-traitement des solveurs spécifiques utilisés par les différents membres du projet.

\subsection{Le Projet ICARE}

Ce projet ICARE [ㄷ] (Ingénierie collaborative et analyse de résultas de calculs éléments finis)

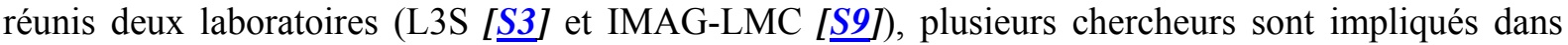
cette action.

Ce projet vise à générer des modèles permettant une meilleure diffusion de l'information au sein des Bureaux d'Etudes (CAO, Calcul...), ainsi que la préparation des données présentées lors des revues et/ou de réutilisation de projets de produits (modèles géométriques, résultats de simulation, etc.). Les données générées sont souvent diverses et variées (modèles, CAO, Eléments Finis, etc.), et issues de logiciels divers (CAO, calcul de structures...).

ICARE se propose de fournir, des moyens de traitement des données, par l'intermédiaire d'un outil de post-traitement, permettant la visualisation interactive des modèles [S2]. Ceci permettra d'élargir l'utilisation, la diffusion, l'analyse ainsi que le transfert en se basant sur des modèles polyédriques issus de calculs Eléments Finis. Il s'agit donc d'un modèle de visualisation de résultats de calcul qui puisse s'insérer dans un contexte d'ingénierie collaborative comme support de dialogue entre différents logiciels (différents métiers). En effet, les modèles actuels requièrent des performances informatiques élevées (stockage et graphique) qui sont incompatibles avec les exigences de dialogue et d'échanges de données au cours du processus de conception. Pour ce faire, il devient donc nécessaire d'adopter des modèles bien adaptés aux capacités d'ordinateurs actuellement utilisés [ $\underline{\mathbf{S}} \mathbf{]}$.

Parmi les principaux objectifs de ce projet on distingue :

- La spécification des structures de données hiérarchiques compatibles avec une approche de simplification multi-critères.

- La confrontation des travaux de recherche menés aux laboratoires $3 \mathrm{~S}$ et LMC à des scénarios industriels..

- La mise en place d'une approche adaptative de décimation multicritères (pondération entre les différents critères).

\subsection{Le Projet Aide à la Conception d'une Chaîne Logistique (laboratoires LAG et GILCO)}

Les auteurs de ce projet [S3] [Sㅇ] soulignent les importantes mutations qu'a subit les l'environnement technico-économique des entreprises et constatent que sous l'effet de la concurrence croissante, les entreprises doivent améliorer leur réactivité. Elles doivent en effet : [드]

- Mettre sur le marché des produits répondant aux attentes fonctionnelles et d'usage des clients (analyse fonctionnelle).

- Délivrer les dits produits au bon client, au moment et à l'endroit convenus, avec les quantités demandées. 
Le premier point relève plus particulièrement de la phase conception. Le second repose sur la mise en place et le management du réseau logistique.

L'objectif de ce projet est de répondre au second point. Pour cela, il est fondamental de mettre en place un réseau logistique efficace qui conduit l'entreprise à répondre à quatre questions principales :

- Quel est le cœur de mon métier, quelles activités de production et de distribution faut-il garder en interne, lesquelles faut-il externaliser?

- Comment réaliser au mieux les activités internes (capacité et organisation des sites de production et des entrepôts, système physique de distribution...)?

- Quels fournisseurs retenir et quel type de relations faut-il avoir avec eux (simple fournisseur, partenariat...)?

\subsection{Le Projet MICA Graph}

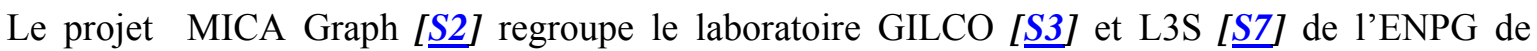
Grenoble en France, et le Département informatique de l'Imperial College à Londres, UK. Il s'intéresse à l'utilisation des nouvelles technologies de l'information et de la communication dans le domaine la conception.

Ce projet part du fait que les informations graphiques (modèle géométrique, croquis, isovaleurs...) jouent le rôle d'un vecteur de communication entre les différents acteurs du processus de conception des produits [드. Ces modèles d'importance capitale, n'ont pas fait l'objet de plusieurs travaux de recherche. A titre d'exemple, peu de travaux ont porté sur la génération des croquis. Or, actuellement de nombreux travaux portent sur l'information textuelle et ont donné lieu à la création d'outils informatique (data-mining, MICA, recherche sur Internet, etc.).

L'objectif principal de la capitalisation du savoir et du savoir-faire n'est plus de figer la connaissance afin de pouvoir en tirer partie plus facilement, mais de capitaliser suffisamment pour être en mesure de créer de nouvelles connaissances. Les travaux du laboratoire GILCO ont montré la possibilité de développer des outils de messagerie capables de supporter des Informations Non Structurées (INS) ou échanges informels, et ce en leur ajoutant des éléments de contexte d'interprétation et en permettant un traitement à posteriori de ces informations. En travaillant ces informations textuelles avec des techniques de Data-Mining, des réseaux sémantiques peuvent être identifiés statistiquement, permettant ainsi d'identifier des problèmes ou constats qui ont donné lieu à des échanges d'informations. Ensuite, les concepteurs peuvent rattacher des significations à ces liens statistiques en associant leurs " connaissances " à ces réseaux sémantiques.

Par ailleurs de nombreuses études de l'activité des concepteurs insistent sur la culture visuelle du travail d'ingénierie. De nombreux auteurs se sont intéressés au rôle des croquis dans la conception notamment dans le travail d'architecture et les phases amont de conception. En effet, les croquis vus comme des INS (Informations Non Structurées) autorisent des définitions partielles des systèmes que ne sont pas autorisées pas les systèmes de CAO actuels. Les croquis sont utilisés à différents niveaux pour des recherches d'idées par des concepteurs mais aussi pour communiquer avec d'autres. L'analyse de l'usage des croquis a montré qu'ils sont un vecteur important d'émergence de nouvelles solutions de conception.

L'objectif de ce projet est de développer un prototype de démonstration des concepts d'un nouveau type de messagerie (MICA Graph.) permettant de capitaliser les échanges entre concepteurs en y associant les INS Graphiques. L'idée est de pouvoir aussi instrumenter les croquis de manière à permettre un traitement a posteriori basé sur des annotions et des commentaires associés au graphisme. Les techniques de Data-mining, concernant le texte, utilisées dans MICA sont alors encore valables. En effet, il sera possible de travailler sur les textes associés aux croquis afin de retracer le contexte dans lequel la décision a été prise. Un des résultats attendus de ce projet devrait être le développement d'une maquette informatique permettant de tester ces hypothèses. Cette maquette devrait permettre ensuite de mettre en place des expériences de conception. Par ailleurs, ce projet devrait donner lieu à 
des échanges approfondis avec le département informatique de l'Imperial College of London dont l'un des domaines de compétence est le traitement statistique des informations aussi bien textuelles que vidéo et audio.

\subsection{Le Projet SG3C}

Le projet SG3C [S2] résulte d'une collaboration entre Schneider Electric et les Laboratoires 3S [S7] et GILCO [ㅈ]. L'objectif est de résoudre des problèmes industriels d'organisation d'entreprises, de méthodes de travail et d'outils associés. Ce projet a aboutit a la proposition d'une méthode et un outil de gestion des multiples calculs générés lors du développement de nouveaux produits techniques.

Dans un contexte d'ingénierie simultanée et distribuée, l'approche proposée permet d'améliorer la coopération entre les analystes (Calcul) et les concepteurs $(\mathrm{CAO})$. Elle fournit également un support à la traçabilité et à la réutilisation des modèles et des démarches de simulations récurrent en conception mécanique. Parmi les concepts proposés, celui de " cas d'école » pour la modélisation et pour la conception, constitue un des éléments clé de l'identification et de la capitalisation des connaissances.

Le projet SG3C a été mené dans le cadre d'une réflexion sur l'intégration du calcul mécanique en conception à partir des trois premiers types des concepts précités. Le point de vue adopté était suffisamment global pour permettre de dégager des solutions originales.

\subsection{Le Projet OSCAR}

Le projet OSCAR [S4] est en quelque sorte une continuation du projet SG3C. Ce projet s'articule autour des trois principaux points suivants :

- Instrumenter et observer le processus de déploiement de SG3C afin d'acquérir un retour d'expérience sur l'usage industriel de l'approche proposée.

- Développer et affiner les concepts de SG3C. En cohérence avec les observations menées sur l'usage industriel, il s'agit de développer de façon plus approfondie des solutions initialement proposées.

- Introduction de nouveaux concepts. En concordance avec les observations de l'usage et le développement des solutions initiales.

Les auteurs de ce projet soulignent l'apport du partenaire RENAULT qui se distingue par des besoins qui marquent un écart significatif en termes de structuration par rapport aux besoins du partenaire initial Schneider Electric.

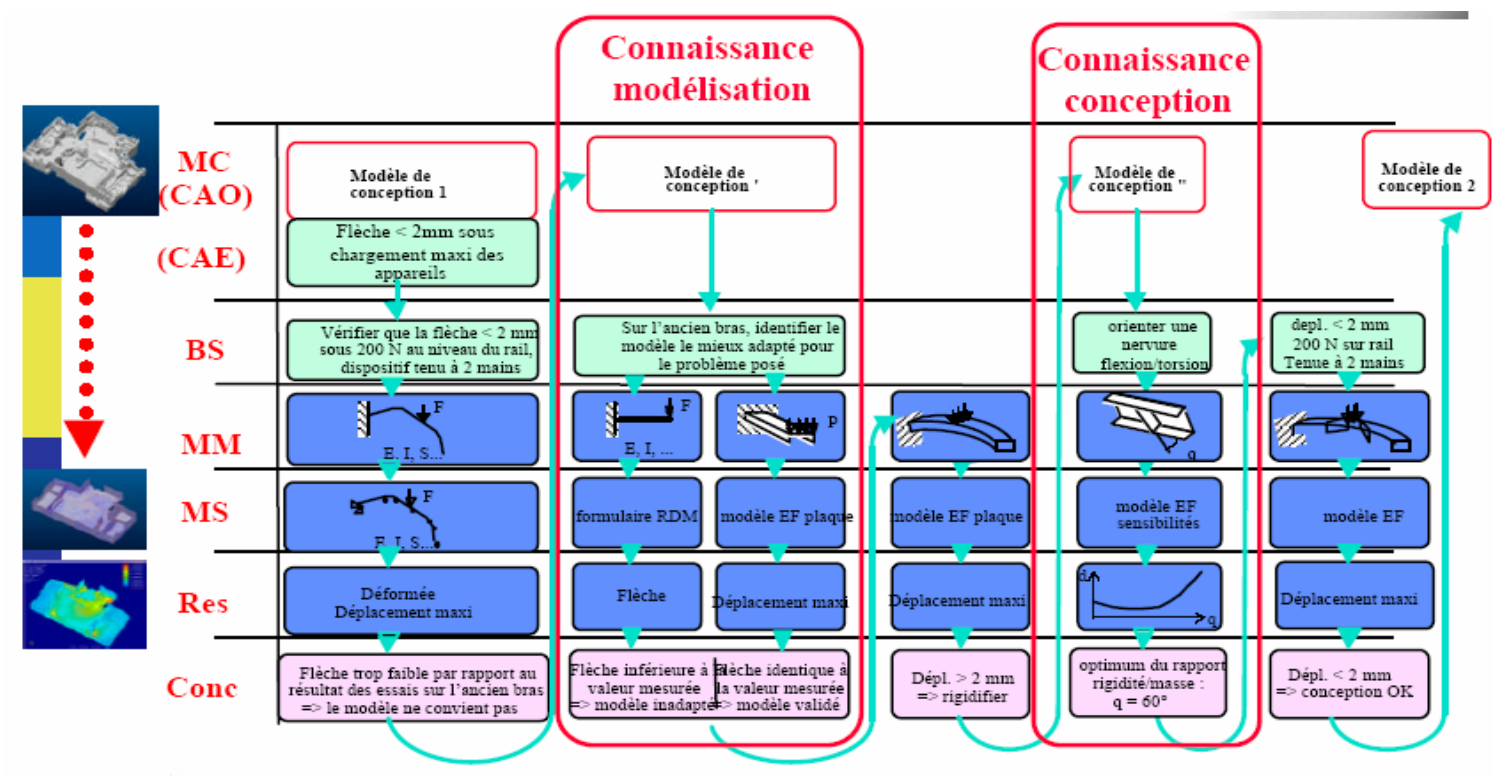

Figure 6 : Processus de conception - simulation dans le projet OSCAR 


\subsection{Le Projet DRAGON}

Le projet DRAGON [S5] (Development of an inteRActive EnGineering Portal for Open Networks) compte seize partenaires universitaires et industriels de l'Europe et de la Chine.

Ce projet propose une plate-forme d'Intégration Numérique permettant de faciliter l'ingénierie simultanée entre plusieurs partenaires industriels éloignés géographiquement les uns des autres. L'intégration numérique est aussi nécessaire au sein de l'entreprise pour assurer la communication entre les outils numériques internes.

Cette notion relative à la co-conception est synonyme de : entreprise étendue, travail collaboratif, plateau virtuel, partage de données techniques. Ces concepts sont devenus une des préoccupations majeures des entreprises. En effet nous assistons à un développement du travail en mode projet qui se caractérise par la mobilisation de compétences multiples et en même temps à un éclatement des structures qui rend le mode projet difficile à mettre en œuvre.

La globalisation des marchés et surtout la réduction des coûts et des délais de développement de nouveaux produits ont conduit au développement des outils de travail collaboratif pour permettre de travailler en mode asynchrone (en temps décalé) et/ou synchrone, et de manière distante tout en assurant la structuration, le suivi, ainsi que la traçabilité des échanges.

\subsection{Le Projet DATAGRID}

Le projet DataGrid [ $\underline{\mathbf{S}}$ ] utilise des applications scientifiques pour montrer comment un système de calcul et de stockage distribué peut être exploité de façon cohérente et intégrée. Des outils et des méthodes seront alors disponibles pour les organisations commerciales qui pourront fournir des produits de qualité industrielle pour supporter et exploiter les environnements de calcul distribué. Le projet consiste à concevoir et développer des solutions et des plates-formes de tests permettant de manipuler des données distribuées, des dizaines de milliers de ressources informatiques (processeurs, disques, etc.), par des milliers d'utilisateurs simultanément.

L'Union Européenne finance le projet à hauteur de 10 Millions d'Euro sur une durée de 3 ans, 2001 à 2003. Le projet est mené et issu du CERN (European Organization for Nuclear Research, Genève Suisse) avec cinq partenaires principaux :

- CNRS (Centre National de la Recherche Scientifique, France),

- ESRIN (European Space Agency's Centre à Frascati, Italie),

- INFN (Istituto Nazionale di Fisica Nucleare, Italie),

- NIKHEF (National Institute for Nuclear Physics and High Energy Physics, Hollande),

- PPARC (Particle Physics and Astronomy Research Council, Angleterre).

Quinze partenaires associés issus du monde de la recherche et de l'industrie participent également à ce projet ; c'est le cas du Commissariat à l'Energie Atomique (CEA) et la société Communication et Systèmes (CS) en France.

Le projet DataGrid est organisé en douze groupes de travail (Work Package) répartis en quatre catégories : Middleware, Infrastructure, Application et Gestion du projet. Les principaux partenaires sont : CEA (France), CS (France), CNR (Italie), CESNET (République Tchèque), IFAE (Espagne), DATAMAT (Italie), CARI (Hongrie), HIP (Finlande), IRST (Italie), IBM (Angleterre), KNMI (Hollande), RPKUH (Allemagne), SARA (Hollande), NFR (Suède), KZZ (Allemagne). Les universités Russes participent également au projet.

Enfin, Plusieurs industriels contribuent de près ou de loin à l'avancement des travaux dans le domaine de l'intégration $\mathrm{CAO} / \mathrm{Calcul}$ que ce soit par financement de projets, mise en place de chercheurs et de matériels de développement ou d'expérimentation ou par la participation dans des 
congrès ou des ateliers de travail (IDMME, MICADO, GRP...). Parmi les principaux partenaires de ce projet, nous citons :

$\begin{array}{ll}- & \text { Autodesk } \\ \square & \text { Bentley Systems } \\ \square & \text { CD Adapco } \\ \square & \text { CETIM } \\ \square & \text { Dassault Aviation } \\ \square & \text { Dassault Systèmes }\end{array}$

$\begin{array}{ll}- & \text { Delcam } \\ - & \text { EADS CCR } \\ \square & \text { EADS Matra Datavision } \\ \square & \text { EDS PLM Solutions } \\ \square & \text { ENSAM } \\ -\quad \text { PSA Peugeot Citroën }\end{array}$

- Fluent France

- Hewlett - Packard

口 IBM

- IFTS

- PI MECA

- Flowmaster France

\author{
- Renault \\ - Simulog \\ - Snecma \\ - The Mathworks \\ - ESI Group
}

\section{Etat de l'art sur les approches d'intégrations existantes}

Dans cette section, nous commençons par poser les différents problèmes relatifs à l'intégration conception / calcul, tels qu'ils apparaissent dans la littérature des travaux de recherche.

Nous savons que la conception et le calcul sont intimement liés. Pour être efficaces, les systèmes support de la conception doivent donc intégrer le calcul mécanique. L'intégration du calcul en conception (conception intégrée) peut cependant prendre plusieurs sens. Pour [Belaziz 99], elle consiste à permettre une meilleure communication entre les logiciels de CAO et les outils d'analyse. Pour [Troussier 99], il s'agit de supporter la traçabilité du processus d'analyse pour une éventuelle réutilisation des résultats et des modèles résultant d'analyses antérieures. Pour [Szabo 96] et [Falco 01], c'est l'évaluation de la qualité des résultats numériques pour apporter de l'aide au cours du processus d'analyse.

Si nous considérons la plupart des travaux de recherche liés à la problématique de l'intégration $\mathrm{CAO} / \mathrm{Calcul}$, nous trouvons que le lien conception - calcul existe selon trois principales approches (Figure 7).

Une première approche concerne le passage des données de la conception (dessins d'ensemble, dessins de définition, ...) vers les outils de simulation (essentiellement le calcul par éléments finis).

Une deuxième approche concerne l'analyse des résultats de calcul et l'évaluation de l'erreur due au modèle de calcul (hypothèses, sollicitation...).

Une troisième approche considère le processus de construction du modèle de calcul (choix des conditions aux limites, choix du modèle de comportement,...).

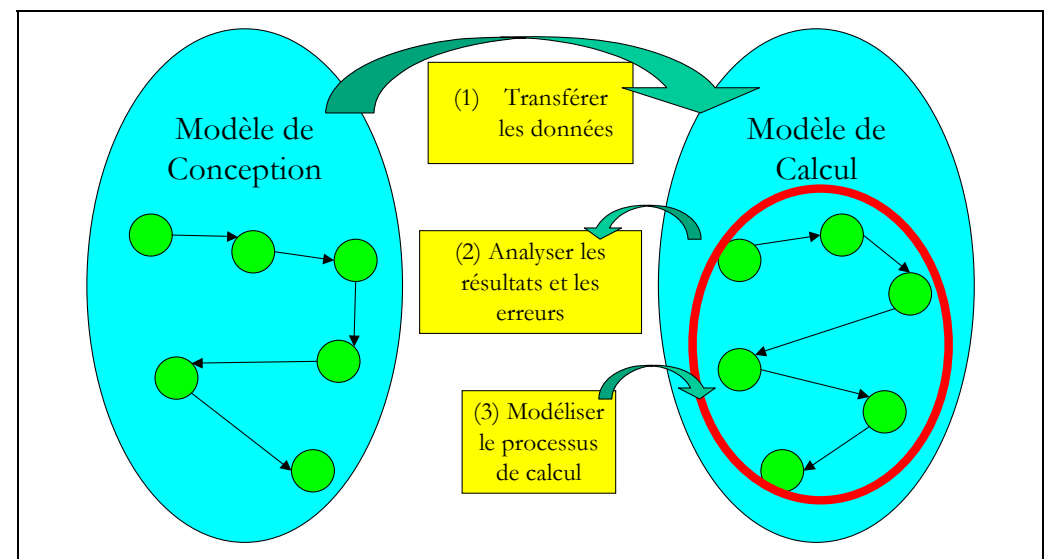

Figure 7 : Interaction Conception/Calcul mécanique

Dans ce qui suit, nous allons recenser les travaux relevant de chacune des approches précitées.

\subsection{Transfert de données de la conception vers le calcul}

Cette approche caractérise les premiers travaux de recherche liés à l'intégration $\mathrm{CAO} / \mathrm{Calcul}$. L'idée est de favoriser le passage automatique (ou pour le moins assisté) du modèle manipulé par le 
système de conception (en général un modeleur géométrique), vers un modèle dédié au calcul (en général par éléments finis).

Dans ce cadre, [Shepard 90] propose un système expert en vue d'assister le concepteur dans les tâches de simplifications géométriques, les choix des modèles de comportement, la modélisation des conditions aux limites et le niveau de discrétisation du modèle géométrique idéalisé qui sera utilisé pour le calcul. Le système est dédié à assister les concepteurs n'ayant pas nécessairement une culture soutenue du calcul. La figure 8 illustre la démarche d'intégration CAO/Calcul selon [Shepard 90].

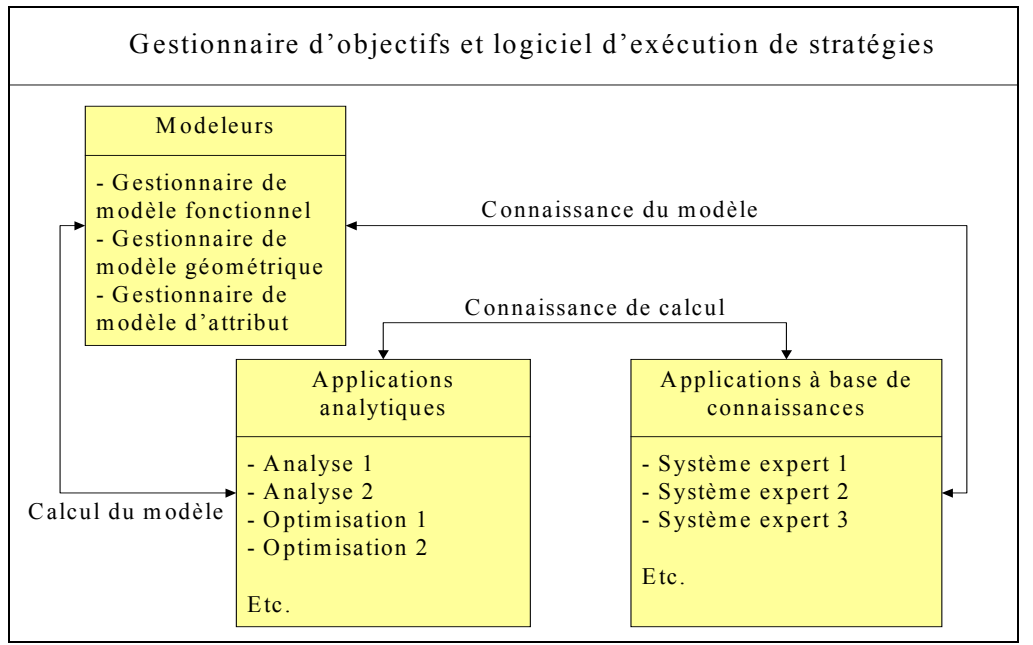

Figure 8 : L'intégration selon [Shepard 90]

Dans le même contexte, [Arabshahi 91] propose un système qui construit les modèles de calcul à partir de critères purement géométriques. Cette approche est contestable, car un modèle mécanique doit prendre en compte les variables technologiques (modèle de comportement, sollicitation, conditions aux limités, ...).

Pour adapter le calcul au contexte (maillage adaptatif), Cuillère [Cuillère 99] propose un modèle de caractéristiques de formes permettant d'identifier, à partir du modèle géométrique du produit, les zones susceptibles d'exercer une influence déterminante sur le comportement mécanique du produit (retrait de matière, congés de raccordement,...). Le maillage est alors affiné dans les régions d'intérêt appelées features de forme, préalablement reconnues. Dans la même logique, [François 98] propose une extension du principe de pré-optimisation du maillage développé dans [Cuillère 93] pour déterminer automatiquement les zones de raffinement. Un re-mailleur tridimensionnel permet un remaillage automatique non plus de toute la pièce, mais de la zone concernée par une modification géométrique ou topologique introduite au cours de la conception. Selon les sollicitations subies par la pièce ou le matériau constituant cette dernière (qui peut être hétérogène), le maillage adaptatif dans les features de forme peut perdre son sens. La figure suivante montre le cas d'une pièce avec un maillage adaptatif dans les raccordements et au voisinage d'un trou. Ainsi, le maillage adaptatif permet de limiter l'augmentation du temps de calcul lorsque l'on recherche un raffinement des résultats d'analyse dans des zones particulières.

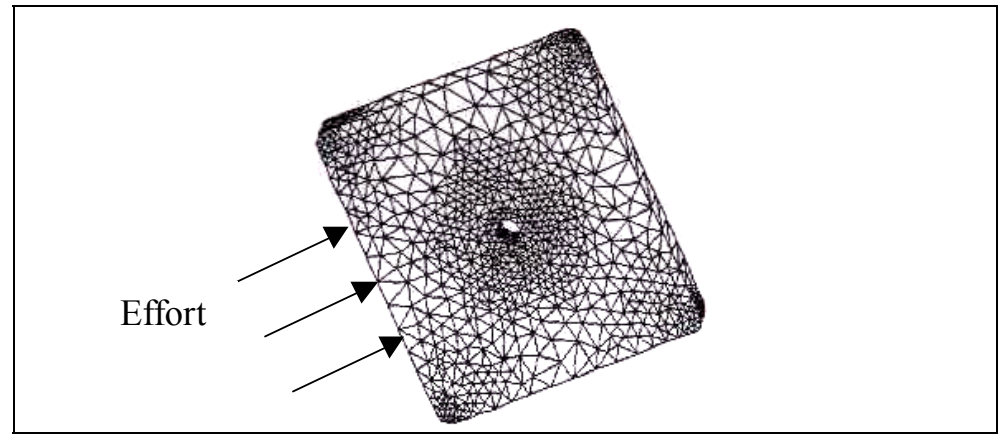

Figure 9 : Maillage adaptatif sur des features de formes 
[Tehari, 99] utilise des caractéristiques de forme pour assurer le passage du modèle géométrique de conception à un modèle idéalisé pour le calcul. Pour cela, il propose d'analyser les modèles issus de la conception d'objets pour générer de nouveaux modèles adaptés aux traitements spécifiques à différents points de vue (calcul, fabrication...). La description des objets est basée sur des briques géométriques élémentaires, définies sous forme BRep. Celles ci sont associées au moyen de règles de composition dépendantes du point de vue métier adopté, pour former des caractéristiques métiers. La démarche comporte une phase d'analyse morphologique suivie d'une phase d'analyse sémantique. La première phase est composée de quatre étapes fondamentales :

- Reconnaissance et identification des caractéristiques morphologiques.

- Extraction.

- Reconstruction des entités modifiées.

- Codification des caractéristiques morphologiques.

La seconde phase consiste à réinterpréter différemment la description intermédiaire générée par la première phase suivant les règles propres au point de vue métier.

[Belaziz 99] propose une approche d'abstraction, basée sur la simplification et l'idéalisation des modèles issus d'un environnement $\mathrm{CAO}$ au moyen des features de forme. Le modèle d'analyse (au sens de la géométrie) est dérivé à partir d'un modèle basé sur des formes caractéristiques. La dérivation du modèle analyse se fait en deux phases :

- Simplification, qui tend à occulter les détails non pertinents pour l'analyse.

- Idéalisation, qui consiste à adapter selon des règles métiers la géométrie simplifiée aux outils de calcul permettant l'application de l'analyse.

Afin de prendre en compte les modifications éventuelles issues de l'analyse, une phase de reconstruction permet de reconstruire un modèle BRep à partir du modèle à base de features de forme en utilisant des macro-opérateurs de reconstruction paramétrique basée sur les opérateurs d'Euler.

[Fine 01] propose une démarche automatique permettant de transformer les modèles CAO pour préparer une analyse par éléments finis. Il utilise des polyèdres pour décrire différents modèles d'analyse (au sens de la géométrie) répondant aux différents besoins. Les multiples représentations permettent de manipuler différents scénarios d'analyse. Par la suite, les simulations de comportement ainsi obtenues sont visualisées comme support pour la validation des solutions de conception. Différentes approches s'inscrivent dans la même logique d'automatisation du passage des données de la conception vers les outils d'analyse mécanique [Sheffer 97], [Dabke 94], [Armstrong 96], [Rezayat 96]. Le principe général est décrit dans la figure 10.

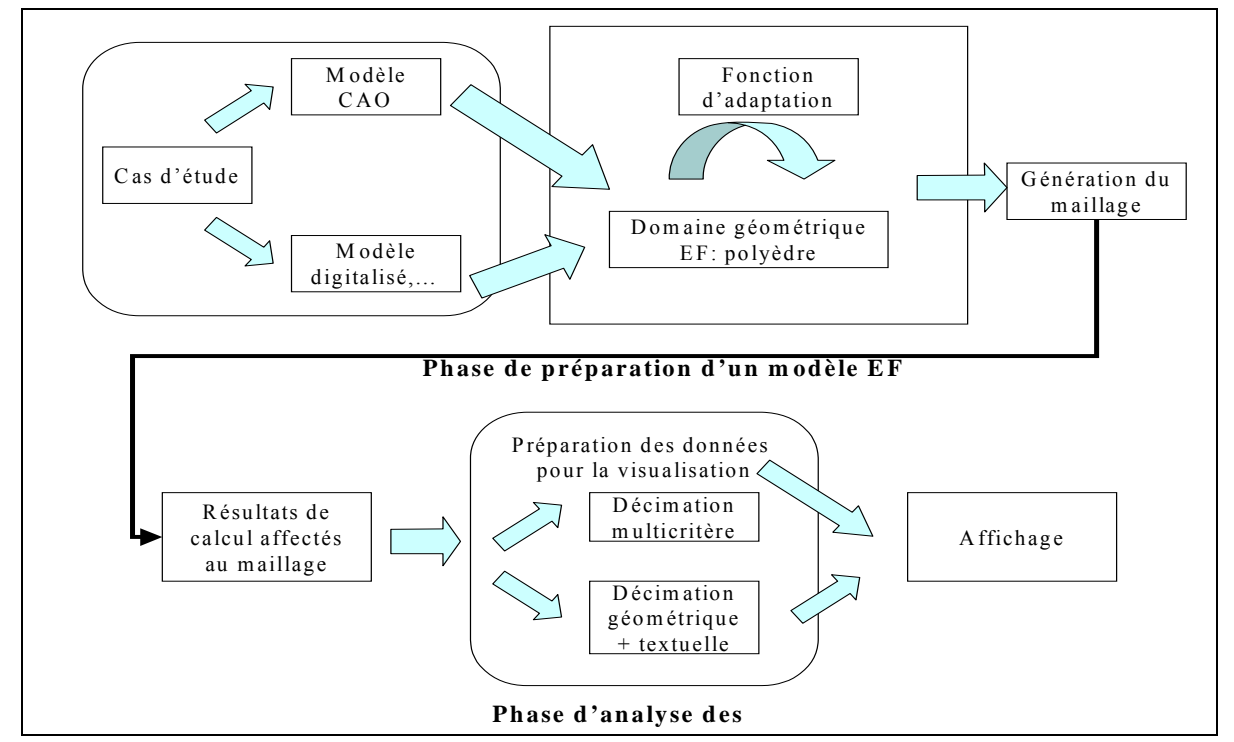

Figure 10 : Schéma du principe de la solution proposée selon [Fine 01]. 


\subsection{Analyse des résultats et erreurs de calcul}

Il existe de nombreuses sources d'erreurs lors de la mise en œuvre des tâches de calcul en conception. Le contrôle de l'apparition de ces erreurs à différents stades du processus de calcul constitue aussi une aide certaine à l'intégration conception/calcul [Vignjevic 98], [Morris 97], etc.

Dans cette logique, [Szabo 96] considère la nécessité d'assurer la qualité numérique des résultats de calcul. L'analyse de l'erreur est utilisée pour diagnostiquer des ambiguïtés dans la construction du modèle de calcul, dues à des hypothèses, des sollicitations, ou des modèles de comportement aberrants. Ainsi, l'auteur porte un jugement sur la crédibilité du modèle de calcul sur la base de l'incertitude qui entache les résultats de simulation du comportement. La figure ci-dessous illustre l'approche systématique de l'analyse de l'erreur proposée par [Szabo 96] tout au long du processus de calcul. Il distingue alors trois classes d'erreurs :

- Les erreurs théoriques, liées aux comportements supposés par l'analyste,

- Les erreurs de modélisation, qui sont soit des erreurs dans la formulation des modèles mathématiques, soit des erreurs d'idéalisation de la géométrie,

- Les erreurs de discrétisation, liées aux méthodes de discrétisation.

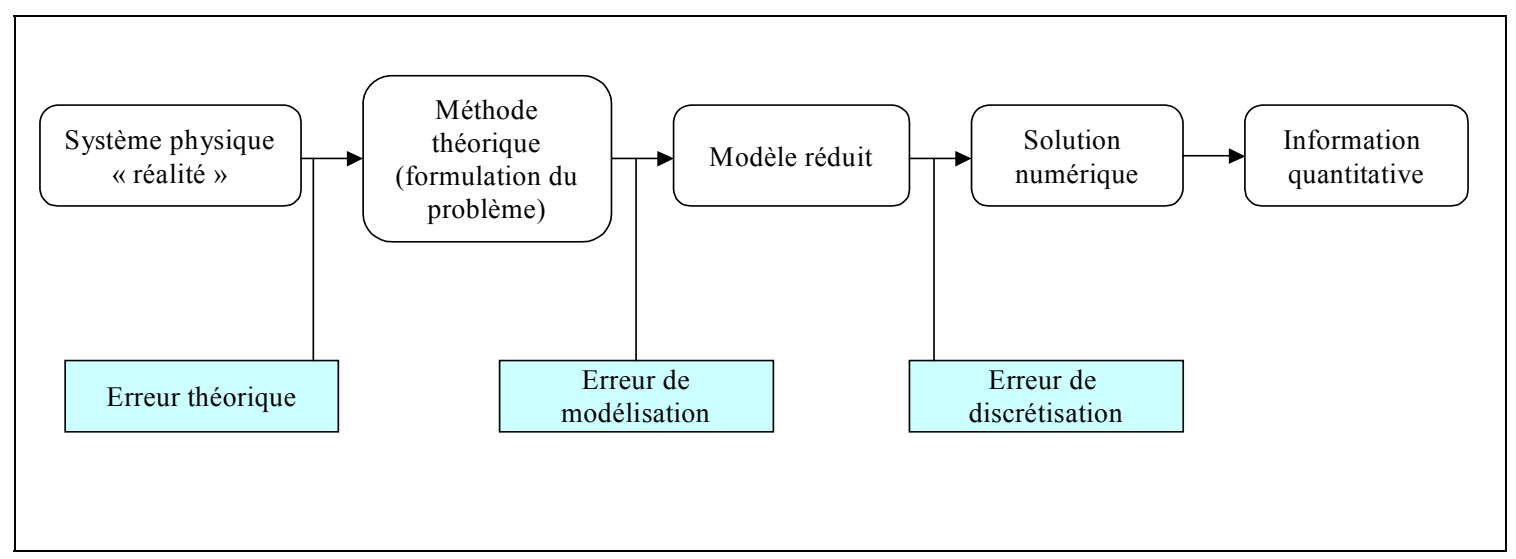

Figure 11 : Modèle d'intégration selon [Szabo 96]

[Falco 01] propose un processus de gestion dynamique des processus d'analyse en vue de contrôler les erreurs commises dans les différentes étapes de l'analyse mécanique. Il suppose que la principale source d'erreurs provient de l'adoption des fausses d'hypothèses. Celles ci se classifient en trois catégories :

- Les hypothèses de modélisation,

- Les hypothèses de simplification,

- Les hypothèses de choix effectués lors de l'analyse.

Cette approche est également suivie par [Fenves 86] et [Breitfield 96]. Or, la correction des erreurs s'avère difficile car il n'est pas possible de reconnaître les hypothèses qui ont été faites au cours de l'analyse.

Dans [Kurowski 95], nous trouvons l'idée d'outils méthodologiques pour assister le concepteur dans le choix des hypothèses de comportement lors de l'analyse par éléments finis, il constate que l'erreur du calcul est souvent due à de mauvais choix au cours de la construction du modèle éléments finis, d'autant plus que cette méthode d'analyse s'impose de plus en plus comme une extension naturelle des modeleurs géométriques.

\subsection{Modéliser le processus de calcul pour le réutiliser}

La notion de calcul est au moins partiellement associée à l'idée d'une procédure formalisée et systématique. Cette procédure est alors virtuellement applicable soit plusieurs fois dans le même contexte mais avec des valeurs différentes, dans le cas d'un affinement de solution, soit dans différents 
contextes similaires, lorsque les problèmes de calcul se posent de manières similaires. De nombreux travaux se sont intéressés à l'exploitation de cette propriété, et ont cherché à modéliser le processus de calcul pour permettre sa réutilisation et ainsi réduire son temps de mise en oeuvre. Cela nécessite de formaliser au moins une partie du cycle de construction d'un modèle de calcul (morphologie, sollicitations, matériau, comportement, modèle d'analyse...).

C'est dans ce contexte que [Lalanne 87] propose, à l'aide d'un système expert, un guide méthodologique basé sur des points-clés permettant de traiter des problèmes spécifiques d'analyse de structures (description du problème, détermination des risques de défaillances, sélection des formulations mathématiques et l'interprétation des résultats). Son travail est basé sur des recherches plus anciennes [Marcal 80], [Fjellheim 83] et [Lagache 84] proposant une aide intelligente dans le choix des logiciels ainsi que des options pour effectuer des calculs de simulation en analyse de structures.

Selon [Fischer 00], le calcul est un outil de validation et de synthèse (Figure 12). La validation consiste à démontrer l'adéquation des choix de conception (forme, matériaux, dimensions...) par rapport à des objectifs comportementaux. La synthèse consiste à activer un raisonnement d'ordre mécanique pour réaliser les choix de conception assurant un comportement donné. Elle concerne la détermination de dimensions associées à une morphologie.

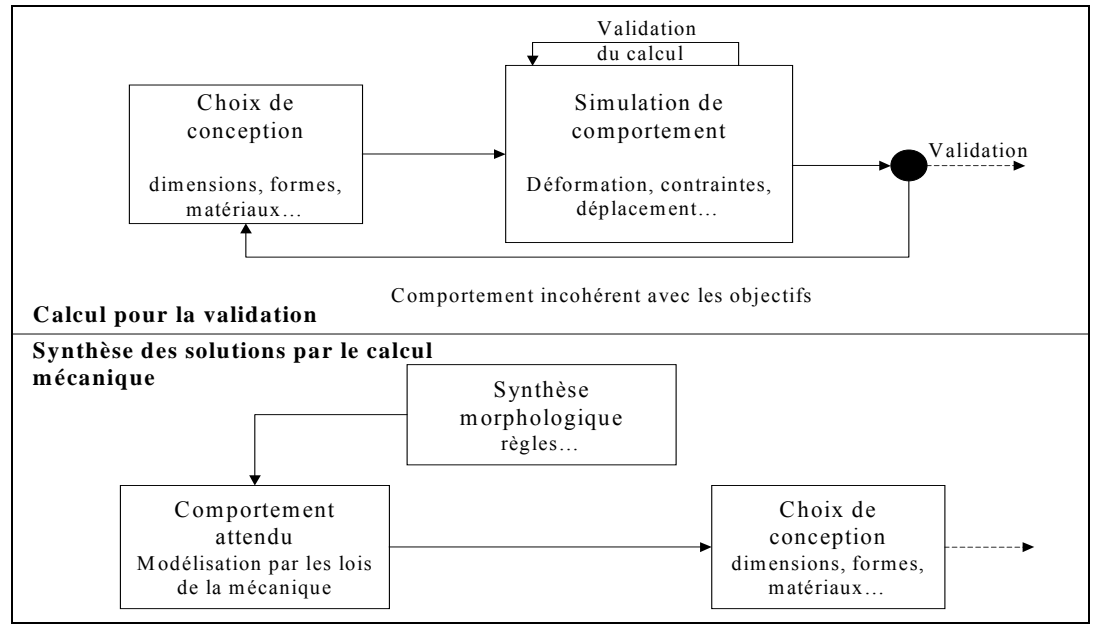

Figure 12 : La synthèse et la validation en conception [Fischer 00]

Fischer suppose que la morphologie existe a priori et propose une démarche de conception inversée, intégrant la synthèse des dimensions des structures. Le système intègre des règles générales de calcul, des règles caractérisant les habitudes professionnelles et des règles sur des domaines d'application spécifiques, telles que les appareils de pression.

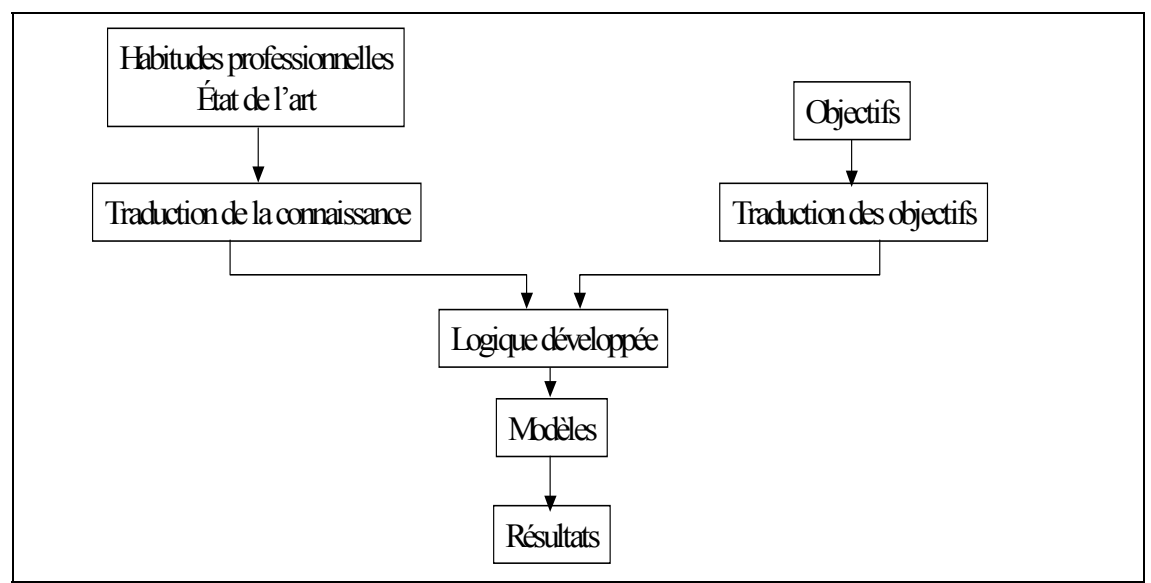

Figure 13 : Modèle intégré selon [Fischer 00] 
Le calcul mécanique est utilisé pour générer, par des techniques de programmation par contraintes, des dimensions optimisées par rapport aux exigences comportementales, sur une forme qui existe a priori. Mais si la forme est inadaptée pour le problème, la démarche conduisant à optimiser les dimensions de cette forme peut conduire à des résultats aberrants. Dans la même logique, [Robert 91] propose une démarche permettant d'optimiser les structures mécaniques vis à vis d'un ensemble de contraintes sur le coût, la géométrie, le matériau, etc. Cette démarche permet de comparer plusieurs configurations possibles de la structure (correspondant à plusieurs itérations du processus d'optimisation couplées avec des simulations de comportement) afin de choisir la version qui réponde au mieux aux exigences comportementales.

[Ghias 01] s'intéresse également à l'optimisation des structures mécaniques au cours de la conception. Dans sa démarche, il part du fait qu'une conception optimisée (au sens de [Robert 91]) peut ne pas respecter des critères de fiabilité. Il intègre alors l'aspect fiabilité et introduit la notion d'optimisation mécano-fiabiliste ou l'optimisation basée sur la fiabilité. L'objectif est d'introduire des critères de fiabilité dans la recherche de la conception optimale des structures afin de trouver le meilleur compromis coût - fiabilité. Des variables de conception et des variables aléatoires (représentent les incertitudes sur le système) sont couplées au moyen de formulations mathématiques pour assurer la convergence simultanée dans les deux espaces, de fiabilité et d'optimisation. Ces différentes approches, si elles permettent effectivement de systématiser le calcul dans certains cas d'applications pour lesquels il existe une expertise, elles s'inscrivent néanmoins dans une démarche globale procédant par essai-erreur, notamment en ce qui concerne la proposition des formes.

[Helary 00], considérant que le cahier des charges est généralement incertain (les critères imprécis...) propose une approche d'aide à la décision en conception, basée sur des techniques de l'intelligence artificielle (logique floue, raisonnement sous contraintes, etc.) en vue de prendre en compte l'aspect incertain des données de conception. Cela permet de trouver des solutions de conception selon plusieurs points de vue (point de vue géométrique, massique, structurel, ...) (figure 14). Un rapprochement des points de vue permet ensuite de trouver la solution qui répond au mieux à tous les points de vue considérés.

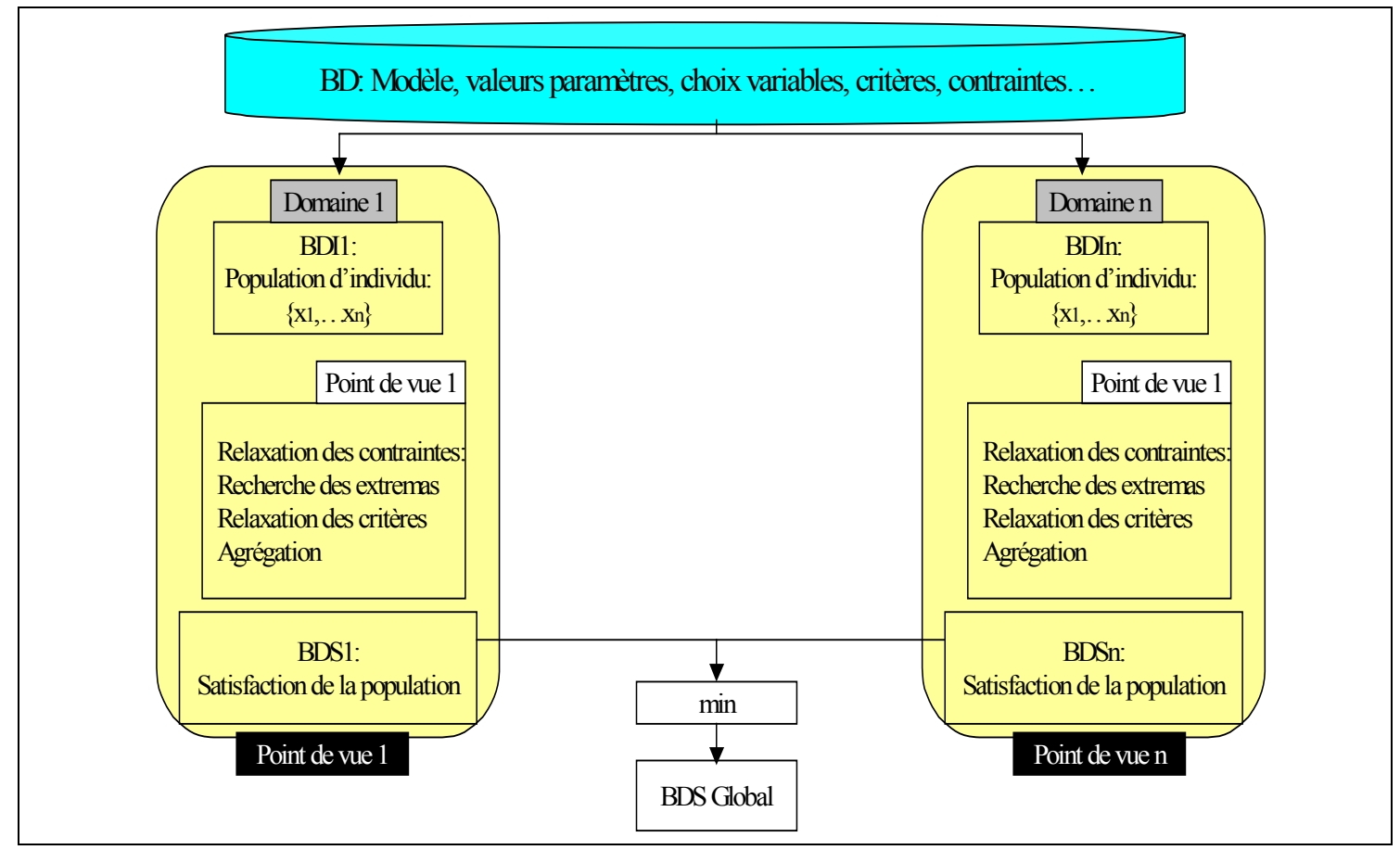

Figure 14 : Approche d'intégration selon [Helary 00]

[Ferraille 98] utilise aussi la logique floue pour modéliser l'expertise du concepteur ainsi que des règles de mécanique nécessaires au calcul de dimensionnement. Cela permet dans un premier temps d'identifier le problème de conception à résoudre, et dans un deuxième temps d'identifier les champs 
de solutions acceptables selon les points de vue considérés (masse, ...). Les travaux de [Bennis 00], [Faltings 92], [Joskowicz 95] et [Sam 95] rentrent dans la même catégorie.

[Troussier 99] considère que le calcul est souvent une reprise d'un calcul existant. Elle propose une méthode pour, d'une part tracer des processus de calcul en cours de conception, en se basant sur des questions, et d'autre part identifier grâce à ces questions les situations dans lesquelles des processus de calcul existants peuvent être réutilisés. Dans la même logique, [Falco 01] s'intéresse au processus d'analyse dans sa globalité en vue de favoriser la capitalisation pour une réutilisation du calcul et un contrôle de l'erreur. Les démarches proposées nécessitent toutefois que le concepteur soit formé en matière de calcul pour mener à bien la construction du modèle, car l'objectif est plus de proposer à un concepteur expert un gain de temps potentiel par la réutilisation future de son expertise, que de faciliter la mise en œuvre de calculs par un mécanicien novice. Notons qu'en l'absence d'expertise suffisante, un utilisateur peut répondre aux questions de façon inexacte et ainsi capitaliser de mauvaises traces ou réutiliser des traces inadaptées.

\section{Synthèse et positionnement de nos travaux.}

L'état de l'art présenté dans cette partie permet de souligner les principaux travaux dans le domaine de l'intégration CAO/Calcul. Dans cette étude bibliographique nous avons présenté non seulement les approches proposé aux laboratoires de recherche, mais aussi celle développés dans le cadre de projets scientifique dans les quels sont impliqués les laboratoires de recherche et les industriels. Ces projets de recherche attestent et argumentent le besoin des industriels en terme d'intégration $\mathrm{CAO} / \mathrm{Calcul}$. En effet, les échanges entre $\mathrm{CAO}$ et Calcul souffrent encore de nombreux problèmes d'intégration.

Cette étude bibliographique montre que :

- L'intégration entre la CAO et le Calcul est un objectif important pour les industriels et les laboratoires de recherche.

- Les systèmes de CAO et de Calcul industriels ne sont pas encore suffisamment intégrés. En effet, même les solutions intégrées (CATIA, Pro/Engineer, SolidWorks) ne permettent pas de répondre aux besoins d'industriels en terme de CAO et de Calcul et il est pratiquement inévitable de passer d'un système à un autre pour valider une conception en calcul.

- Les erreurs de calcul sont souvent liées à des problèmes de modélisation. Le contrôle de l'apparition de ces erreurs à différents stades du processus de calcul constitue aussi une aide certaine à l'intégration Conception/Calcul.

- Les problèmes d'échange de données sont au centre du débat sur l'intégration $\mathrm{CAO} / \mathrm{Calcul}$. Les normes d'échange de données (IGES, STEP, etc.) souffrent encore de problèmes de fiabilité de transfert et de compatibilité entre eux.

- Les informations requises pour le calcul ne sont pas bien représentées dans les modèles de CAO support de l'activité de conception.

- La gestion du processus de conception (CAO, Calcul, FAO, etc.) n'est pas compatible avec les tendances actuelles (ingénierie simultanée). La modélisation des processus de calcul peut faciliter de façon significatives leur réutilisation.

- Les outils de calcul actuel ne permettent pas de guider le concepteur lors des tâches de calcul (choix du modèle, choix du type de calcul, interprétation des résultats, etc.).

- Les échanges entre CAO et Calcul ne sont pas bidirectionnels (On peut aller de la CAO vers les outils de Calcul mais pas dans le sens inverse).

Nos travaux sur l'intégration du calcul dans le processus de conception se distingue des différentes propositions présentées dans l'étude bibliographique par le fait que nous ne considérons pas que la problématique d'intégration $\mathrm{CAO} / \mathrm{Calcul}$ réside uniquement dans la résolution des difficultés de transfert des informations entre les modèles géométriques et les modèles d'analyse. 
De ce fait, dans une première approche, nous remettons en cause l'organisation des systèmes de $\mathrm{CAO}$ actuels qui présupposent qu'on ne peut calculer qu'après avoir défini le modèle géométrique. Ensuite, nous proposons des entités orientées calcul (features de calcul) permettant de structurer les calculs en conception et d'aider les concepteurs lors du dimensionnement des solutions envisagées en $\mathrm{CAO}$. Nous abordons ensuite les possibilités d'utilisation des résultats de calcul dans l'environnement $\mathrm{CAO}$ (reconstruction des modèles $\mathrm{CAO}$ à partir des résultats de calcul élément finis). Une plateforme de développement orienté intégration $\mathrm{CAO} /$ Calcul sera également présentée.

La partie suivante de ce rapport présente une synthèse des approches proposées. 


\section{PARTIE III :}

Synthèse des Travaux de Recherche sur l'Intégration

CAO/Calcul. 


\section{PARTIE III}

\section{Synthèse des Travaux de Recherche sur l'intégration CAO/Calcul.}

\section{Introduction}

Cette partie du mémoire est consacrée à la présentation des principaux résultats de recherche émanant de nos travaux dans le domaine de l'intégration $\mathrm{CAO} / \mathrm{Calcul}$. Nous commençons par un bref rappel des modèles proposés dans la thèse de doctorat [BenAmara 98] et nous enchaînons ensuite avec une présentation détaillée des travaux de recherches issues des actions d'encadrement de thèses et mastères en génie mécanique. Ces actions ont été menées au Laboratoire de Génie Mécanique de l'Ecole Nationale d'Ingénieurs de Monastir en collaboration avec, d'une part, le Laboratoire d'Automatique et de Mécanique Industrielles et Humaines (LAMIH, UMR CNRS 8530) de l'Université de Valenciennes en France, et d'autre part, le Département de Génie Mécanique de l’Université du Québec à Trois Rivières au Canada.

Nous profitons de la présentation en détail des résultats de recherche issues de chacune des approches proposées pour présenter également les résultats scientifiques publiés et les travaux soutenus (Mastères et Thèses) dans le cadre de nos activités d'encadrement.

\section{Approche d'intégration CAO/Calcul par enrichissement du modèle de produit.}

L'état de l'art sur les principales approches de l'intégration $\mathrm{CAO} / \mathrm{Calcul}$ montre que les facteurs clés de l'intégration $\mathrm{CAO} / \mathrm{Calcul}$ sont le modèle de produit, le modèle de processus et les modèles d'échange de données [BenAmara 98].

Dans le cadre de la thèse de doctorat [BenAmara 98], effectuée en collaboration entre le Laboratoire de Génie Mécanique (MA-05) de l'ENI de Monastir (Tunisie) et le LAMIH-UMR CNRS 8530 de l'Université de Valenciennes, notre contribution à l'intégration de la composante calcul dans le processus de conception a essentiellement porté sur ces trois aspects.

Ci-dessous, nous présentons brièvement le modèle de produit et le modèle de processus proposés pour pallier aux problèmes d'intégration $\mathrm{CAO} / \mathrm{calcul}$. Nous détaillons la structure et les concepts de ces modèles et nous explicitons leurs aptitudes, d'une part, à modéliser le produit et le processus aux différents niveaux de modélisation, et d'autre part, à gérer la propagation des contraintes géométriques et technologiques générées par les différentes tâches de calcul et d'évaluation. Nous présentons également les techniques de communication envisagées pour supporter l'échange d'informations entre le système support de l'activité de conception et les outils d'analyse et d'aide à la conception.

\subsection{Structure du modèle proposé}

Le modèle de produit est une abstraction du produit réel. Il doit permettre, d'une part, la structuration des relations entre les différents objets utilisés au cours du processus de conception (modèle conceptuel), et d'autre part, la description fonctionnelle, géométrique, topologique et technologique du produit (modèle descriptif). Il doit également permettre de décrire le comportement du produit en fonction du niveau de modélisation considéré (modèle de comportement) [Shah 96] [Banningen 95].

Le modèle de produit proposé est constitué d'un ensemble de concepts et de relations entre ces concepts. Les concepts modélisent les différentes entités permettant de décrire le produit au cours du processus de conception, alors que les relations décrivent la structure de données qui le caractérise. Il est structuré par un réseau de relations basé sur trois concepts génériques : Ensemble, Composant et Liaison, indépendants du domaine de modélisation produit. Ainsi, une liaison "pivot" du domaine 
technologique peut apparaitre dans le domaine technique comme un ensemble de différents composants (axe, alésage, goupille, patin) dont les relations sont également exprimées dans le domaine technologique [BenAmara 98]. La structure globale de ce modèle est présentée dans la figure ci-dessous sous forme de diagramme objet OMT [Booch 00]].

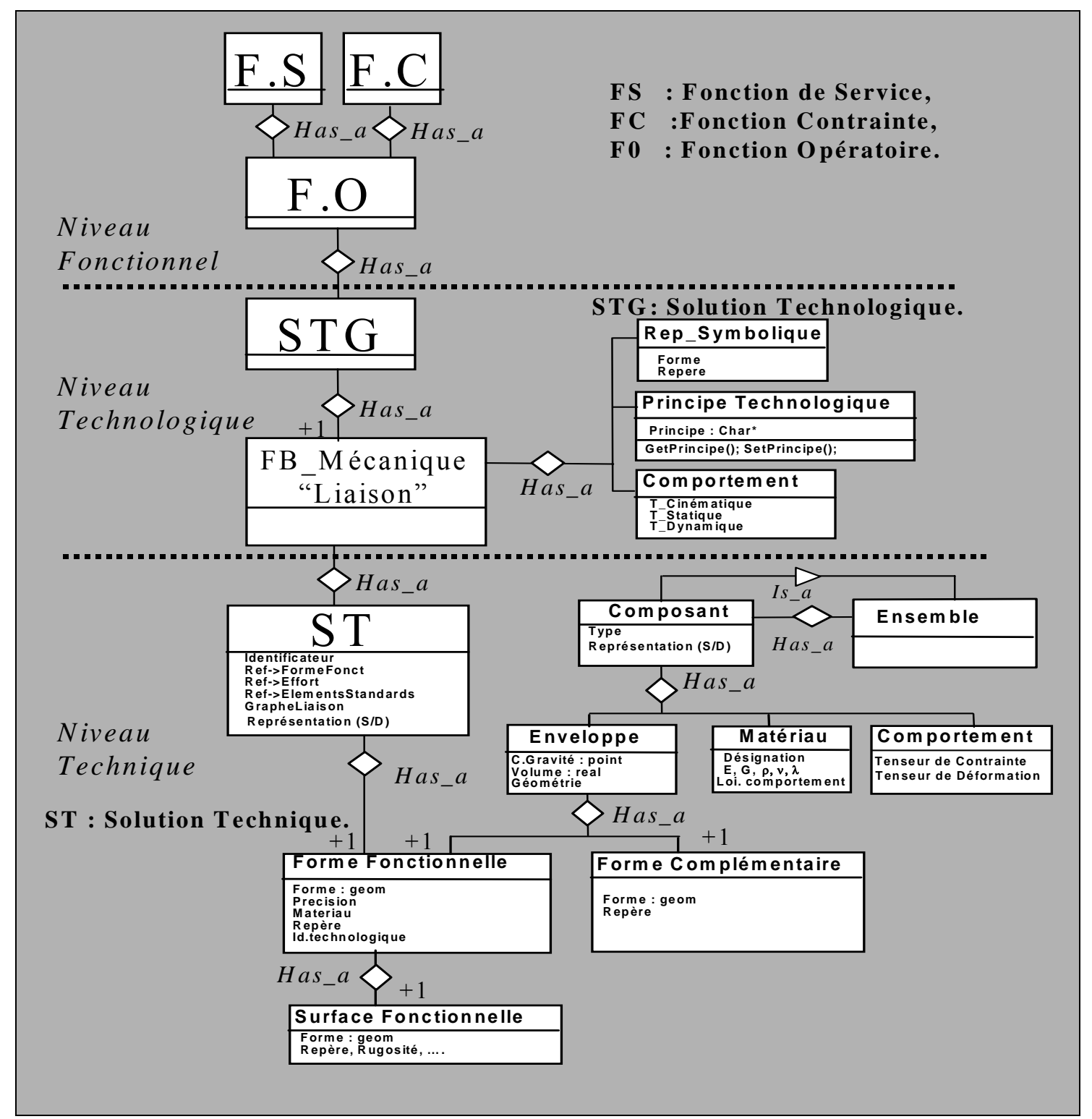

Figure 15. Diagramme objet OMT des modèles technologiques et techniques

Ce diagramme spécifie les concepts de chacun des nivaux de modélisation, technologique et technique, ainsi que les relations entre ces concepts. Au niveau fonctionnel, les fonctions de service et les fonctions contraintes se traduisent par des fonctions opératoires. Cependant, ces dernières, s'expriment au niveau technologique, selon le mécanicien, par des solutions technologiques construites à partir de fonctions de base mécaniques. Ces fonctions de base sont caractérisées par une représentation symbolique, un principe technologique et un comportement. Au niveau technique, à chaque fonction de base correspond une solution technique caractérisée par des formes fonctionnelles appartenant à différents composants mécaniques. Chaque composant est caractérisé par une enveloppe, un matériau et un comportement. L'enveloppe est constituée de formes fonctionnelles et de formes complémentaires.

Dans ce qui suit, les principaux concepts du modèle proposé sont détaillés, en se limitant aux deux domaines technologique et technique caractérisés par une forte interaction entre les processus de conception et de calcul. 


\subsubsection{Les concepts du domaine technologique}

A ce niveau de modélisation, l'interprétation mécanicienne des exigences fonctionnelles est représentée en terme de solutions technologiques. Les principaux concepts de ce domaine sont illustrés dans le tableau 1.

\begin{tabular}{|l|l|}
\hline \multicolumn{1}{|c|}{ Concept } & \multicolumn{1}{|c|}{ Définition } \\
\hline Solution technologique & $\begin{array}{l}\text { Une solution technologique décrit une transformation } \\
\text { d'énergie et/ou de mouvement ou une structure. elle } \\
\text { exprime les relations entre les différents éléments } \\
\text { mécaniques et leur environnement. Une solution } \\
\text { technologique est représentée de manière symbolique. }\end{array}$ \\
\hline Fonction de base & $\begin{array}{l}\text { Une fonction de base assure de manière autonome et } \\
\text { spécifique à un métier, la réalisation d'une fonction } \\
\text { particulière du produit ou de l'un de ses composants. } \\
\text { Les fonctions de base spécifiques au point de vue } \\
\text { mécanique concernent les liaisons mécaniques } \\
\text { glissière, pivot, rotule, encastrement ...) }\end{array}$ \\
\hline Principe technologique & $\begin{array}{l}\text { Un principe technologique permet de préciser différents } \\
\text { moyens candidats pour satisfaire une fonction de base } \\
\text { sans présumer de formes ou de matériaux particuliers. }\end{array}$ \\
\hline
\end{tabular}

Tableau 1 : Les concepts du domaine technologique

\subsubsection{Les concepts du domaine technique}

Les solutions techniques associées aux concepts du domaine technologique ont pour but d'associer une description matérielle aux modèles technologiques. Cette dernière est caractérisée par une morphologie et des paramètres technologiques et dimensionnels. Les principaux concepts du domaine technique sont illustrés dans le tableau 2.

\begin{tabular}{|c|l|}
\hline Concept & \multicolumn{1}{|c|}{ Définition } \\
\hline Solution technique & $\begin{array}{l}\text { Ce concept modélise l'ensemble des solutions physiques } \\
\text { (forme, matériau, ... permettant de matérialiser les } \\
\text { principes technologiques associés aux fonctions de base } \\
\text { définies dans le modèle technologique. }\end{array}$ \\
\hline Forme fonctionnelle & $\begin{array}{l}\text { Une forme fonctionnelle est une forme géométrique, } \\
\text { engendrée par une solution technique associée à l'une des } \\
\text { fonctions de base du modèle technologique }\end{array}$ \\
\hline $\begin{array}{c}\text { Forme } \\
\text { complémentaire }\end{array}$ & $\begin{array}{l}\text { Une forme complémentaire ne matérialise pas une fonction } \\
\text { de base, elle ne participe qu'indirectement aux fonctions } \\
\text { technologiques du produit. Elle doit satisfaire les } \\
\text { contraintes de résistance, de fabrication et d'assemblage de } \\
\text { ces composants. }\end{array}$ \\
\hline Enveloppe & $\begin{array}{l}\text { Ce concept caractérise l'espace occupée par un composant } \\
\text { élémentaire. L'enveloppe de chaque composant mécanique } \\
\text { est constituée par des formes géométriques orientées par } \\
\text { rapport à la matière et raccordées les unes aux autres. }\end{array}$ \\
\hline Matériau & $\begin{array}{l}\text { Le matériau définit les caractéristiques physico-chimiques } \\
\text { d'un composant mécanique (module d'élasticité, résilience, } \\
\text { etc.) }\end{array}$ \\
\hline Comportement & $\begin{array}{l}\text { Ce concept permet de décrire le comportement des } \\
\text { composants via les tenseurs de contraintes et de } \\
\text { déformations }\end{array}$ \\
\hline
\end{tabular}

Tableau 2. Les concepts du domaine technique

L'ensemble des concepts précédemment exposés (technologique et technique) révèle des significations universelles permettant d'unifier le langage entre les acteurs intervenant dans les deux processus de conception et de calcul. La sémantique associée à chacun de ces concepts assure néanmoins une interprétation selon d'autres points de vue, notamment la fabrication et l'assemblage. 
Dans les paragraphes suivants, nous présentons la partie mise en œuvre informatique des modèles proposés.

\subsection{Mise en œuvre informatique : F-CAD, un prototype de Conception Fonctionnelle}

La mise en œuvre informatique des modèles proposés a été réalisée sur la plate-forme de développement orientée objet CAS.CADE de Matra DataVision. L'application est composée de quatre modules : le module interface utilisateur, le module entités de bas niveau, le module entités de haut niveau et le module de communication avec les outils de calcul.

La maquette réalisée est capable de traiter des exemples de conception permettant de montrer les interactions entre les deux processus de conception et calcul. A travers des photos d'écran présentées dans les figures 3 , les principales fonctionnalités offertes par cette maquette sont montrées.
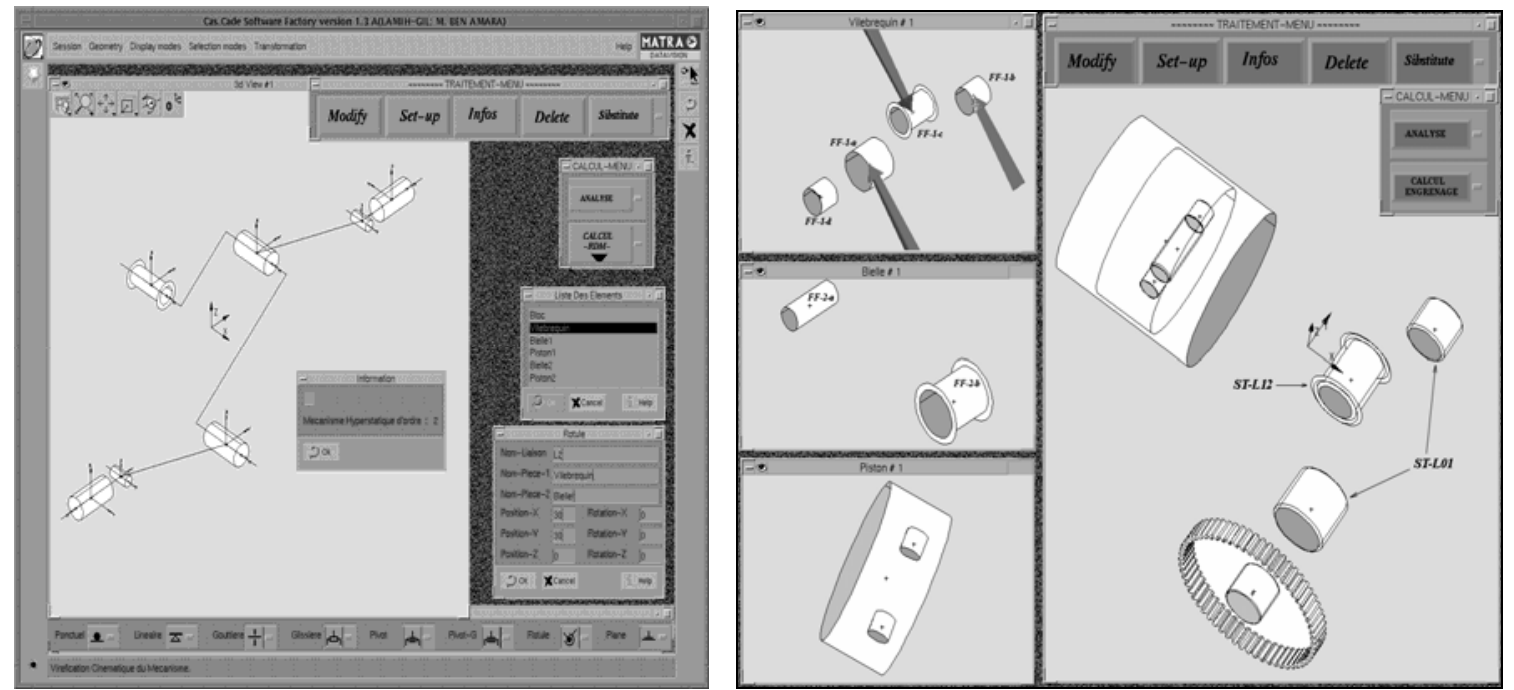

Figure 16. Modélisation d'un produit mécanique sous F-CAD.

Le dimensionnement fonctionnel des solutions techniques est assuré par les modules de calcul développés sous F-CAD. Ces derniers permettent le dimensionnement des clavettes, des engrenages et le calcul des vis CHC. Cependant, la validation locale ou globale de la solution fait appel à des outils de calcul basés notamment sur la méthode des éléments finis. Le système F-CAD permet de générer l'ensemble des informations géométriques et technologiques requises à ce type de calcul.

\subsection{Apport du modèle proposé}

Le modèle proposé illustre une approche originale de conception fonctionnelle intégrant la composante calcul dans le processus de conception. La maquette présentée permet d'illustrer les principales fonctionnalités de ce modèle :

- Aptitude à supporter les informations requises au calcul mécanique.

- Aptitude à gérer la propagation des contraintes géométriques et technologiques engendrées par les tâches de calcul et d'évaluation.

- Aptitude à échanger les informations avec les outils d'analyse en conservant la sémantique associée. 


\section{Approche d'intégration CAO/Calcul par FEATURES de calcul.}

Dans cette partie, l'interopérabilité des processus de conception et de calcul est envisagée autour d'entités méthodologiques appelées Features de calcul qui jouent le rôle de vecteur de communication entre les tâches de conception et de calcul. Ce travail est réalisé dans le cadre de la thèse de Nizar Aifaoui que nous avons co-encadrée avec des collègues du Laboratoire de Génie Mécanique (MA-05) de l'ENI de Monastir (Tunisie) et des collègues du Laboratoire LAMIH-UMR CNRS 8530 de l'Université de Valenciennes en France (voir Annexe II : Fiches Encadrement Scientifique). L'objectif premier de ce travail est de rehausser sensiblement le niveau d'interopérabilité des processus de conception (modèle produit de conception $\mathrm{CAO}$ ) et des processus d'analyse mécanique (modèles de dimensionnement ou d'évaluation), avec l'objectif de formaliser des outils génériques et modulaires permettant d'établir entre ces processus parallèles des liens bidirectionnels à faible composante géométrique [Aifaoui 03].

\subsection{Définition : Features de calcul}

L'objet des Features de calcul est ainsi d'assister le concepteur dans sa démarche de modélisation et de résolution de problèmes de calcul typiques fréquemment rencontrés au cours des phases amont de la conception. Il s'agit notamment des niveaux de modélisation technologique (dans lesquels sont validés les principes de solution) et technique (dans lesquels sont matérialisés les composants à concevoir et les composants standards). Ce concept a émergé suite à une étude expérimentale de plusieurs cas de conception. Celle-ci a montré l'existence d'une généricité tant au niveau des modèles et des données utilisés, qu'au niveau de la démarche de modélisation puis de résolution des problèmes de calcul (scénarios de calculs reproductibles dans des cas de conceptions différents).

Une feature de calcul est définie comme une entité générique paramétrée caractérisant une classe de calcul mécanique. Comme les features définies par Shah [Shah 91], les features de calcul sont porteuses des propriétés de Généricité : elles structurent d'une façon unique les concepts et modèles correspondant aux diverses classes de problèmes de calcul; et de Modularité : elles sont organisées de façon modulaire pour mieux s'adapter à la caractérisation flexible de problèmes variés.

Une Feature de calcul est exprimée sous la forme d'un sextuplet, associant trois modèles de données (mécanique, simulation, interprétation) et trois relations de gestion de cohérence entre ces modèles . Elle intègre principalement :

- Le Modèle Mécanique (MM), qui est une représentation d'un ou de plusieurs éléments de la solution de conception établie par le concepteur à partir d'hypothèses liées à la forme et aux comportements mécaniques de ces éléments, et à la nature des sollicitations (ou conditions aux limites) auxquelles sont soumis ces éléments.

- Le Modèle de Simulation (MS), qui est un modèle calculatoire du modèle mécanique. Il précise les formules ou plus généralement les procédures de calcul, analytiques ou numériques, susceptibles de produire une évaluation des comportements du système étudié.

- Le Modèle d'Observation (MO), qui se présente comme un ensemble de variables d'observation du comportement mécanique du produit que le concepteur souhaite analyser pour évaluer le comportement de la solution retenue ou de ses éléments. Il permet aussi de valider les hypothèses faites au niveau de la construction du modèle mécanique. Ces variables d'observation sont déterminées à partir de la simulation. 


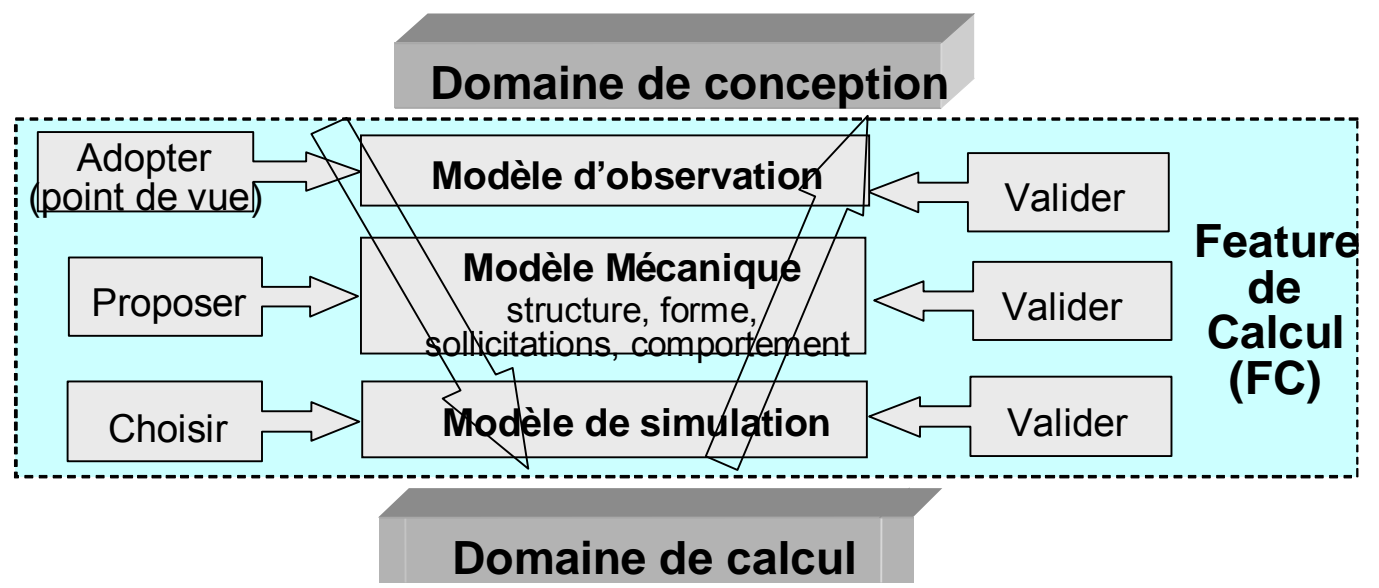

Figure 17 : Features de Calcul et démarche de résolution d'un problème de calcul

\subsection{Organisation des features de calcul}

L'analyse des cas de conception a conduit à formaliser un catalogue de features de calcul (Figure 18) relatif au domaine de la mécanique des solides. Dans ce catalogue, les différentes features sont organisées sous forme d'arborescence, classées selon les domaines de modélisation technologique et technique, et selon les principales variables d'observation (par exemple en cinématique : la vitesse, l'accélération, ...). Les branches " cinématique » et « statique » associées au domaine de modélisation technologique, et la branche "résistance » associée au domaine de modélisation technique ont été plus particulièrement développées.

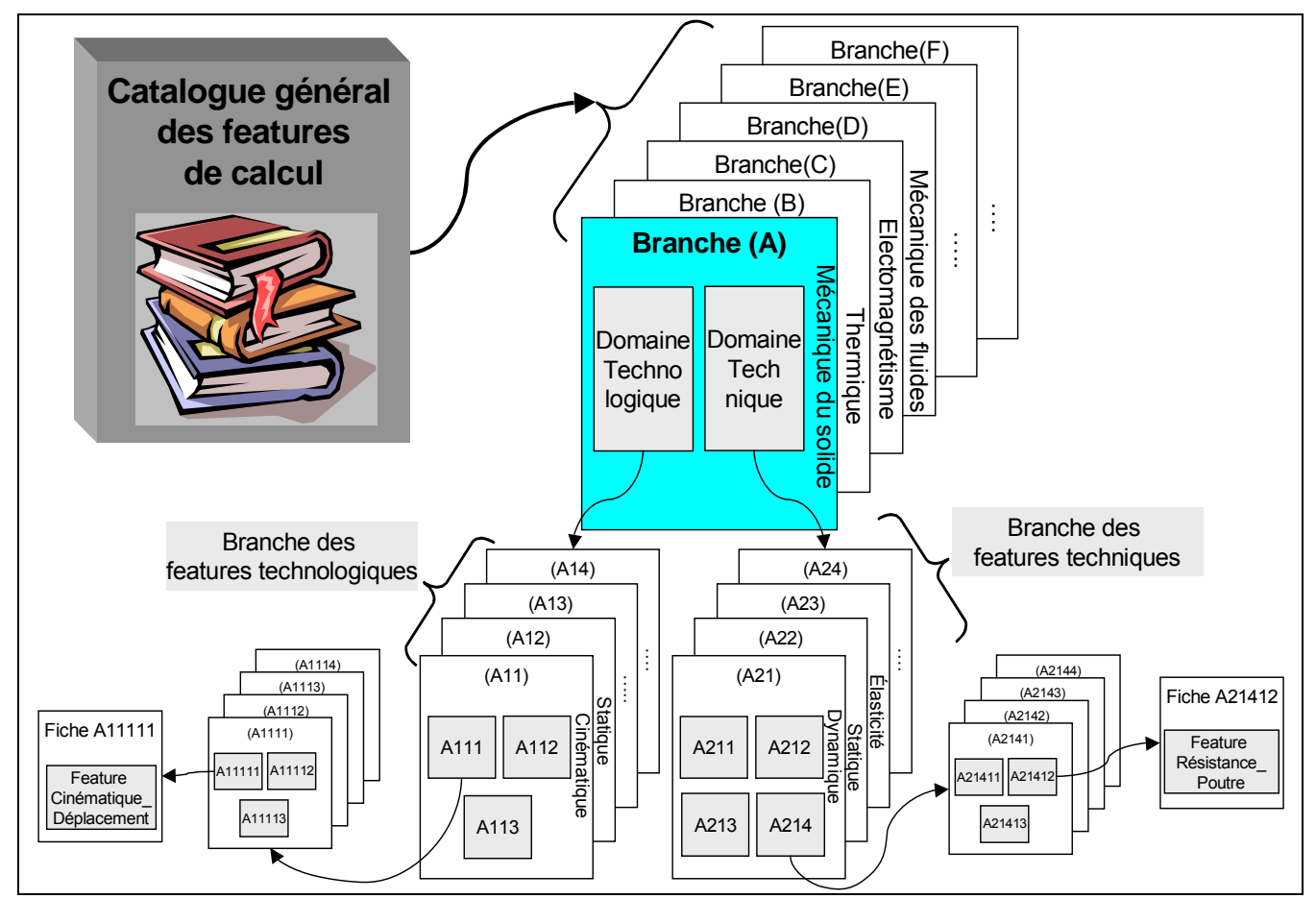

Figure 18 : Catalogue des features de calcul

De par son organisation, le catalogue de features assiste et guide le concepteur dans la démarche de choix et de spécification des informations conduisant au bon déroulement des tâches de calcul visant à dimensionner ou valider une proposition de conception. En effet, à partir d'un contexte de conception donné (du domaine technologique ou technique), le concepteur commence par spécifier le type de calcul à mener (Etude cinématique, ou étude statique) en fonction du problème de calcul. La spécification de la cinématique permet l'orientation du concepteur vers le catalogue des features cinématiques. Tandis que la spécification de l'étude statique le conduit vers le catalogue des features 
statiques. Une fois cette étape franchie, le concepteur rentre dans les détails de la modélisation du calcul en spécifiant les hypothèses de modélisation mécanique (structure, composants technologique, interfaces, sollicitations), le modèle de simulation et le modèle d'interprétation. Ces étapes correspondent à la formalisation du problème de calcul, mais en même temps, cela correspond au parcours de l'arbre cinématique ou statique proposé, qui s'achève par le choix d'une feature de calcul particulière correspondant au problème posé.

\subsection{Mise en œuvre et utilisation des features de calcul}

La spécification détaillée (au moyen des formalismes UML: Unified Modeling Language) [Booch 00] des principaux constituants du système d'information en charge de gérer cette méthodologie de conception est d'ores et déjà bien engagée. Mais le chemin à parcourir pour prototyper un système opérationnel est encore long, car il nécessite de développer de manière intégrée un environnement de conception fonctionnelle, un catalogue relativement complet de features de calcul, des mécanismes d'instanciation, d'édition, d'indexation et de réutilisation de ces features, et bien sûr d'y interfacer une gamme suffisante d'outils de calcul chargés de mettre en auvre les modèles de simulation sélectionnés.

La figure 19 représente le diagramme de classes UML du catalogue des features caractérisant le champ « mécanique du solide».

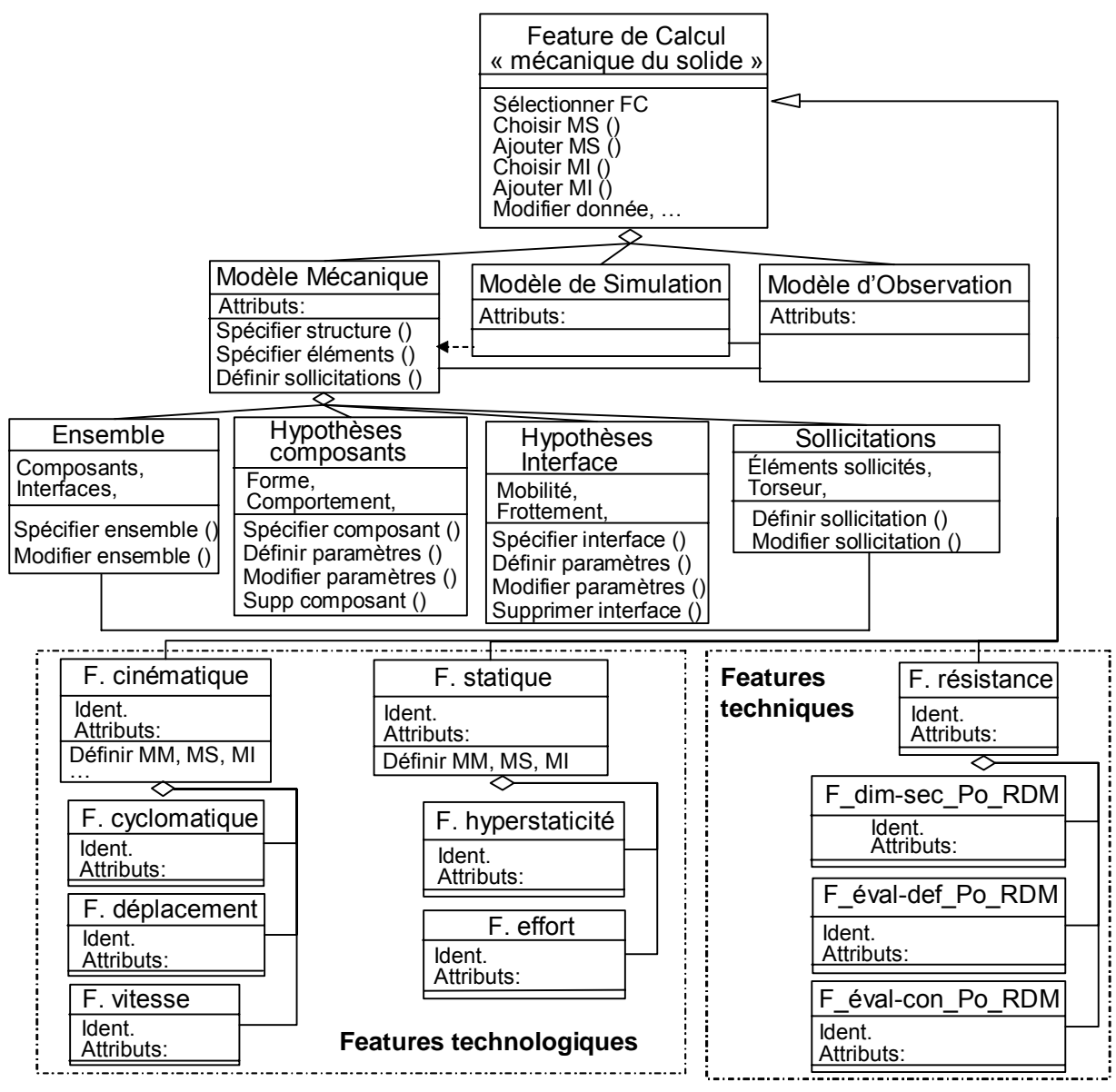

Figure 19 : Diagramme de classes du système proposé

Le diagramme de séquences ci-dessus précise la chronologie des messages circulant entre les objets de la feature de calcul permettant son exploitation pour résoudre une tâche de calcul. L'intérêt majeur de ce type de diagramme est de représenter non seulement l'enchaînement des séquences de déroulement des tâches de modélisation permettant : le calcul, la modification, ou le re-calcul, mais aussi les informations manipulées par chaque séquence. 
Le diagramme ci-dessous décrit l'enchaînement des messages nécessaires pour mener un calcul cinématique (ou statique). Le concepteur commence par spécifier le type de calcul à mener (Etude cinématique, ou étude statique) en fonction du problème de calcul. La spécification de la cinématique permet l'orientation du concepteur vers le catalogue des features cinématiques. Tandis que la spécification de l'étude statique le conduit vers le catalogue des features statiques. Une fois cette étape franchie, le concepteur rentre dans les détails de la modélisation du calcul en spécifiant les hypothèses de modélisation mécanique (structure, composants technologique, interfaces, sollicitations), le modèle de simulation et le modèle d'observation. Ces étapes correspondent à la formalisation du problème de calcul, mais en même temps, cela correspond au parcours de l'arbre cinématique ou statique qui s'achève par le choix d'une feature de calcul particulière correspondant au problème posé.

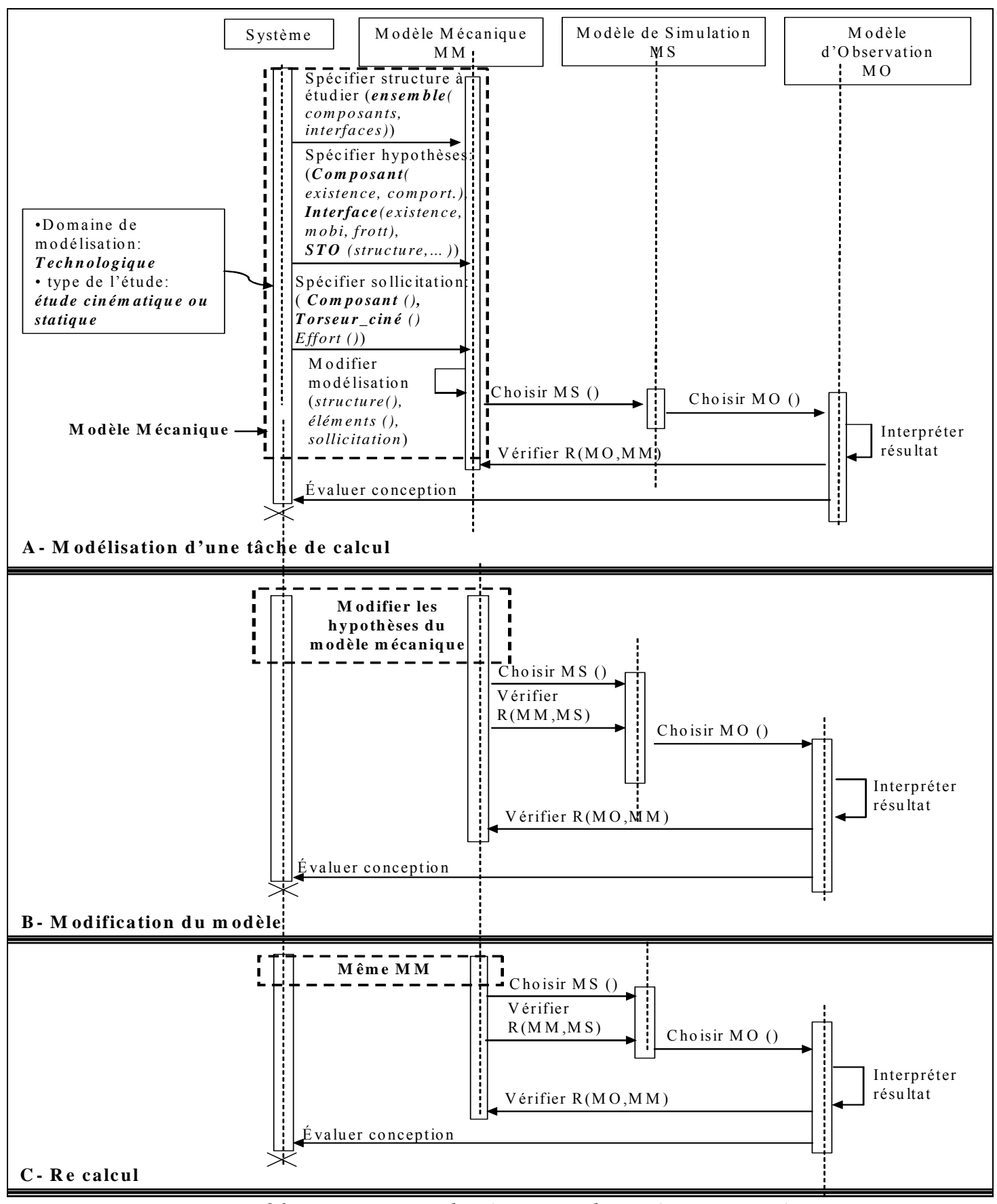

Figure 20. Diagrammes de séquences du système proposé

Ce diagramme représente donc le cas où le résultat du calcul (cinématique ou statique) n'est pas accepté après observation et interprétation des résultats de simulation. Le concepteur est amené à 
modifier les hypothèses de modélisation. Par conséquent, il va remonter l'arbre de construction. Cela peut conduire à l'utilisation de la même feature de calcul, avec des valeurs différentes ou la spécification d'une autre feature caractérisée par d'autres hypothèses de modélisation.

Le diagramme de séquences (figure 20) représente le cas où le résultat de la simulation est accepté, mais, le concepteur envisage d'observer le comportement mécanique de la solution de conception au moyen d'un autre modèle d'observation représentant un autre point de vue du comportement. Dans ce cas, le concepteur garde les mêmes hypothèses de modélisation tout en choisissant un autre modèle d'observation, ce qui correspond à la spécification d'une autre feature de calcul caractérisée par un modèle d'observation différent.

\subsection{Synthèse sur le modèle proposé : Apport et Limites.}

Le concept de feature de calcul est présenté comme un outil de conception sémantiquement riche et jouant le rôle d'un vecteur de communication entre le domaine de la conception et le domaine de calcul. Ce concept est porteur de propriétés de modularité et de généricité. La modularité provient du découpage du modèle de feature en trois modèles sous-jacents (MM, MS, MO), ce qui rend possible toute construction de plusieurs triplets de modèles candidats à la résolution des problèmes de calcul. La généricité réside dans sa structure conceptuelle, qui se manifeste par l'existence d'un macroprocessus. Celui-ci permet, d'abord, d'identifier les problèmes de calcul pertinents à partir d'un contexte de conception et de construire le modèle mécanique adéquat, ensuite, de choisir un modèle de simulation et de déterminer le modèle d'interprétation du comportement à partir de la simulation, puis, d'évaluer le modèle mécanique à partir de l'interprétation et enfin d'évaluer la proposition de conception. Ce processus de calcul permet de modéliser différentes tâches de calcul, à plusieurs niveaux de modélisation de produit. Cela donne au concepteur un cadre méthodologique réutilisable pour la résolution des problèmes de calcul récurrents en conception.

Le protocole d'évaluation basé sur le développement d'un exemple concret de conception (un dispositif de fermeture pour machine de moulage par injection) [Aifaoui 03] a mis en évidence les principaux avantages de cette approche, que l'on peut résumer ainsi:

- L'utilisation du catalogue constitue une assistance au concepteur, qui bien que non expert en analyse est en mesure de construire, de manière adaptative au contexte, le modèle de calcul adéquat.

- Cette démarche assure la traçabilité des boucles d'analyse, et favorise la réutilisation de processus de calcul formalisés dans des contextes similaires, mais avec des valeurs différentes. Ceci engendre un gain dans le temps de calcul et réduit le coût de la conception.

- Grâce à cette méthode, le concepteur devient plus réactif car il a la possibilité de mener des boucles locales de modification ou de re-calcul (changement d'hypothèses de modélisation, choix de nouveaux modèles d'interprétation...).

Cependant, certaines limites ont été relevées et nous ont amené à mettre en avant les points suivants :

- Le catalogue des features ne couvre que le champ de la mécanique du solide et ne comporte que les quelques fiches que l'analyse expérimentale nous a permis de mettre à jour. L'analyse expérimentale d'autres cas de conception portant sur d'autres champs de calcul (thermique, électromagnétisme, fluide...) permettra d'enrichir les modèles et les données recueillis dans notre étude, et de créer d'autres features de calcul, soit pour compléter le catalogue existant, soit pour créer d'autres catalogues.

- Les traces de calcul décrites par des chemins parcourus dans le catalogue, pour être réutilisables dans un système automatisé de traitement de l'information, doivent être codifiées d'une manière adéquate qui n'a pas été approfondie dans ce travail. Nous pensons que la codification et l'indexation d'une trace de calcul (notamment, relativement à d'autres traces de calcul), en vue de sa sélection et de sa réutilisation 
pertinente dans un contexte différent constitue un thème de recherche nouveau, issu du présent travail.

- Enfin, il est manifeste que ces propositions n'ont pas fait l'objet d'une implémentation informatique. L'implémentation dans un langage objet autour d'un noyau de modélisation géométrique s'en trouvera ainsi grandement facilitée.

\subsection{Retombées scientifiques des approches proposées.}

Nos travaux sur l'intégration $\mathrm{CAO} / \mathrm{Calcul}$ basée sur le concept de Feature de Calcul ont fait l'objet de la thèse de Nizar Ifaoui soutenue en régime de cotutelle de thèse en collaboration avec le Laboratoire de Génie Mécanique de l'Ecole Nationale d'Ingénieurs de Monastir et le Laboratoire d'Automatique et de Mécanique Industrielles et Humaines (LAMIH, UMR CNRS 8530) de l'Université de Valenciennes en France. (Voir annexe II, Fiches d'Encadrement Scientifique : Thèse de Nizar Ifaoui).

Cette collaboration a bénéficié d'un financement de mobilités de chercheurs dans le cadre des actions d'échanges DGRST/CNRS : Projet DGRST-CNRS, code 01/R 1103 : (Annexe III)

Ces travaux ont abouti à plusieurs publications scientifiques nationales et internationales (voir Annexe I : Fiches des Publications Scientifiques) :

- N. Aifaoui, A. BenAmara, A. Dogui, "Interopérabilité des processus de conception et de calcul »; Journal Européen de systèmes automatisés ; Vol 38-3-4 (2004) pp 443-464.

- B. Louhichi, N. Aifaoui, A. BenAmara, V. François, D. Deneux ; «Intégration numérique et interopérabilité des processus de CAO et de Calcul », XVII Congrès Français de Mécanique ; UTT -Troyes, France, 29 Août - 02 septembre 2005.

- N. Aifaoui, D. Deneux, R. Soenen, "Feature based interoperability between design and analysis processes", accepté dans Journal of Intelligent Manufacutring, à paraître en Janvier 2006.

- N. Aifaoui, A. BenAmara ; «Les Features de calcul : Un modèle générique d'intégration $\mathrm{CAO} / \mathrm{Calcul} »$ Premier Congrès International Conception et Modélisation des Systèmes Mécaniques : CMSM2005; 23-25 Mars 2005 ; Hammamet - Tunisie.

- N.Aifaoui, A. BenAmara, D. Deneux " Towards a management model of design and mechanical analysis processes based on feature concept"; $5^{\text {eme }}$ conférence internationale sur la conception et la fabrication intégrées en mécanique IDMME 04, Bath, UK avril 2004.

- N. Ifaoui, D. Deneux, A. BenAmara, R. Soenen, A. Dogui, "Mechanical analysis process modelling based on analysis features", IEEE international conference SMC' Hammamet, Tunisie, (6-9 oct 2002)

- N. Ifaoui, D. Deneux, A. BenAmara, R. Soenen, A. Dogui, "CAD/ Analysis integration based on generic model of mechanical analysis process", $4{ }^{\text {ème }}$ conference internationale sur la conception et la fabrication intégrées en mécanique: IDMME'02, IFMA, CLERMONTFERRAN, France, (14-16-mai 2002) 


\section{Approche d'intégration $\mathrm{CAO} / \mathrm{Calcul}$ par reconstruction du modèle $\mathrm{CAO}$ à partir des résultas de calcul par éléments finis.}

La construction d'un modèle numérique était (et est encore dans certains cas) la phase la plus fastidieuse et la moins productive lors de l'emploi des outils de modélisation numérique de conception (CAO), calcul (Solveurs éléments finis) et de fabrication (FAO). En ce qui concerne les deux phases conception et calcul, le passage d'un environnement à un autre se fait le plus souvent à travers des formats standards (IGES, STEP, STA, etc.) ou spécifiques (traducteurs d'un logiciel à un autre). En pratique le concepteur propose des solutions de conception en CAO, puis il procède au transfert de ces modèles géométriques dans un environnement dédié calcul en vue de valider ou de remettre en cause la solution de conception proposée.

La prise en compte des résultats de calcul dans l'environnement de conception CAO pourra pallier un certain nombre de problème de communication entre la $\mathrm{CAO}$ et le Calcul. En effet, il est envisageable de rendre exploitable les résultats de calcul (déformations) dans l'environnement de $\mathrm{CAO}$ en vue d'étudier les assemblages dans leur configuration déformée. Notons qu'actuellement la récupération des résultats de calcul (iso-valeurs, tableaux de valeurs etc.) dans l'environnement de $\mathrm{CAO}$ est actuellement à la charge du concepteur. Afin de remédier à ces problèmes, plusieurs travaux de recherche ont été menés dans l'objectif d'améliorer les échanges bidirectionnels entre les outils de $\mathrm{CAO}$ et de calcul [François 98].

Ce sujet fait l'objet d'une thèse de doctorat (Borhène Louhichi) que nous co-encadrons avec des collègues du Laboratoire de Génie Mécanique (MA-05) de l'ENI de Monastir (Tunisie) et des collègues du Département de Génie Mécanique de l'Université de Québec à Trois Rivières (Canada).

Ce travail tente d'améliorer le niveau d'intégration entre le domaine de la CAO (modèle géométrique) et le domaine du calcul (calcul éléments finis). Le but est de réinjecter dans l'environnement de conception $(\mathrm{CAO})$, le modèle $\mathrm{CAO}$ reconstruit à partir des résultats éléments finis (Maillage déformé) afin de donner au concepteur des moyens quantitatifs de validation ou de remise en cause des propositions de conception.

D'autre part, la reconstruction du modèle CAO à partir de l'analyse Éléments Finis est devenue de plus en plus importante en conception intégrée, notamment pour la simulation en temps réel des processus de conception et/ou fabrication de produit mécanique (Simulation du processus d'emboutissage par exemple). En effet, lorsque l'on simule un comportement élastoplastique d'une pièce dans un mécanisme, les déformations permanentes inhérentes à ces études entraînent une déformation du maillage qui peut devenir non valide ou de mauvaise qualité. La convergence de la solution numérique est alors bloquée parce que les données ne sont plus valides. Dans ce cas, le maillage doit être donc reconstruit (remaillage) entre certaines étapes de la simulation. Autour des déformations permanentes, non seulement le maillage doit être recalculé mais les conditions aux limites doivent aussi être repositionnées sur la nouvelle forme du modèle.

Dans un contexte de conception intégrée, les conditions aux limites sont directement saisies sur le modèle $\mathrm{CAO}$, c'est pourquoi les déformations du modèle éléments finis doivent être transmises au modèle CAO pour repositionner de nouveau les conditions aux limites.

L'étude des problèmes d'assemblage et de simulation numérique du fonctionnement est actuellement (outils de $\mathrm{CAO}$ actuels) abordée en considérant la configuration non déformée des pièces (solide rigide avec géométrie nominale). La reconstruction des modèles $\mathrm{CAO}$ à partir des résultats de calcul tente de lever cette limitation et rend possible la visualisation et la simulation du comportement d'un assemblage à l'état déformé (état normal de fonctionnement) et la détection des éventuelles interférences entre les pièces de l'assemblage étudié. Ces dernières sont indétectables à l'état non déformé. 


\subsection{Hypothèses et éléments de base du modèle proposé}

Dans le cadre de ce travail (thèse de Borhène Louhichi) nous nous sommes limités aux comportements des systèmes mécaniques (mécanique des solides déformables). L'étude sera donc limitée au comportement en petites et en grandes perturbations (élastique et élastoplastique).

Ces hypothèses se conduisent aux considérations suivantes (Figure 21) :

- Petites perturbations : Ni changement de géométrie, ni changement de topologie.

- Élasticité linéaire : Changement de géométrie sans changement de topologie.

- Elastoplasticité : Changement géométrique et topologique.

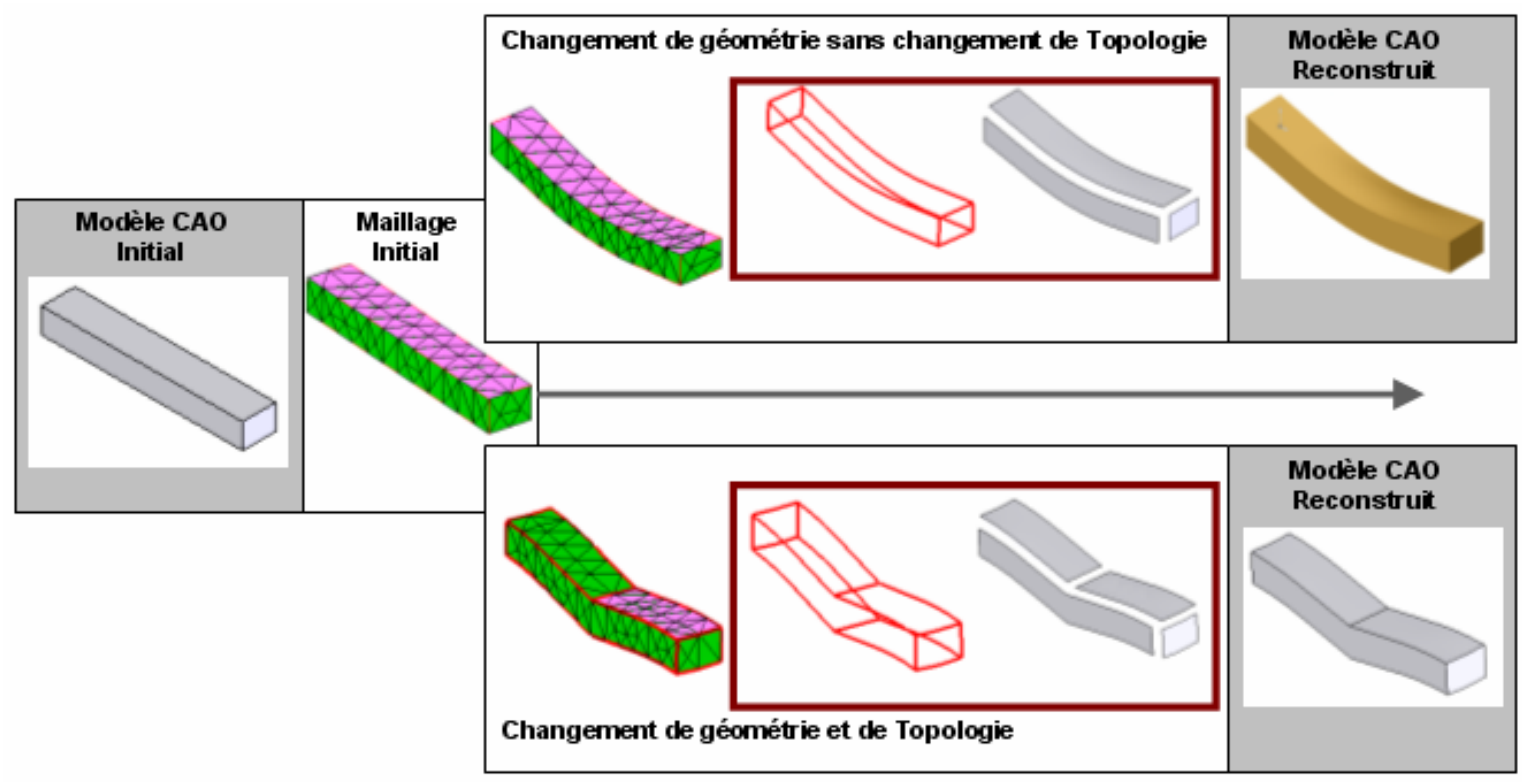

Figure 21 : Modèle initial et modèle déformé.

La reconstruction du modèle CAO est basée sur le modèle BREP (Boundary REPresentation) [Mantyla 98]. Le modèle BRep a été choisi car :

- Il est unique en le comparant au modèle CSG (Constructive Solid Geometry).

- Il est disponible sur la majorité des modeleurs géométriques.

- Il englobe aussi bien des informations géométriques (la nature des surfaces) que topologiques (les liens entre les différentes entités).

\subsubsection{Conservation de la Géométrie et la Topologie}

Cela correspond au cas de petites perturbations, dans le quel le modèle CAO déformé conserve sa géométrie et sa topologie. Dans la figure 22, une poutre soumise à une sollicitation de traction, conserve sa géométrie et sa topologie même si sa dimension latérale change. En fonctionnement réel, cela peut influencer les ajustements et les jeux fonctionnels existant dans un mécanisme. La prise en compte de cette déformation dans le modèle $\mathrm{CAO}$ permettra de prévoir les cas de disfonctionnement.
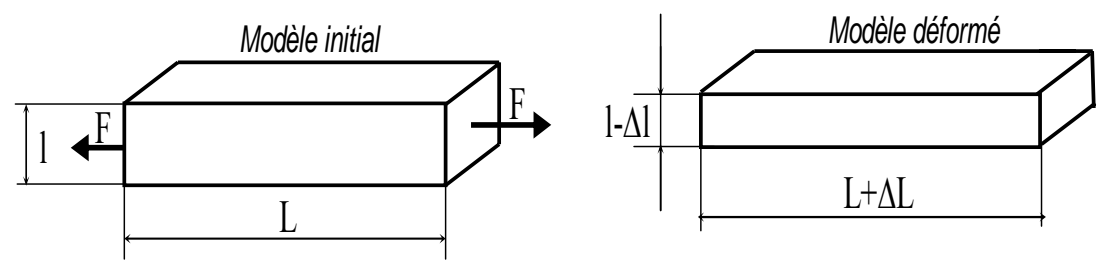

Figure 22 : Changement des dimensions de modèle 


\subsubsection{Changement de Géométrie sans changement de Topologie}

Cela correspond au cas de grandes déformations, dans le quel le modèle CAO déformé change de géométrie tout en conservant sa topologie. Dans la figure 22, une poutre soumise à une sollicitation de type flexion simple change de géométrie (la nature des différentes faces et arêtes change) et conserve sa topologie (la connexion des faces reste la même). La non prise en compte de ces aspects en fonctionnement réel engendrera des problèmes de performance voire de disfonctionnement.

\subsubsection{Changement de Géométrie et de Topologie}

Cela correspond au cas de grandes déformations, dans le quel le modèle CAO déformé change de géométrie et de topologie. Dans la figure 22, la poutre soumise à une sollicitation de type cisaillement, change de géométrie et de topologie (apparition de nouvelles arêtes et faces). Le modèle CAO reconstruit n'est pas conforme au modèle CAO initial. Cette information permet au concepteur d'améliorer la proposition de conception (matériaux, dimensions...) afin de corriger cet écart.

Dans ce que suit, un algorithme général de reconstruction du modèle CAO à partir de résultats éléments finis sera présenté. Cet algorithme sera exprimé dans chacun des cas de figure introduits cidessus.

\subsection{Algorithme général de reconstruction des modèles CAO à partir d’un maillage déformé.}

Dans cet algorithme, il s'agit, dans un premier temps d'identifier la triangulation ou le maillage surfacique à partir de maillage déformé. Cela correspond au maillage relatif à chaque face du modèle. Cela permettra, dans une étape intermédiaire, de reconstruire les surfaces porteuses des faces. Ensuite, les contours, les arêtes et les sommets seront reconstruits par intersection des faces. Cela conduira, enfin, au modèle CAO déformé.

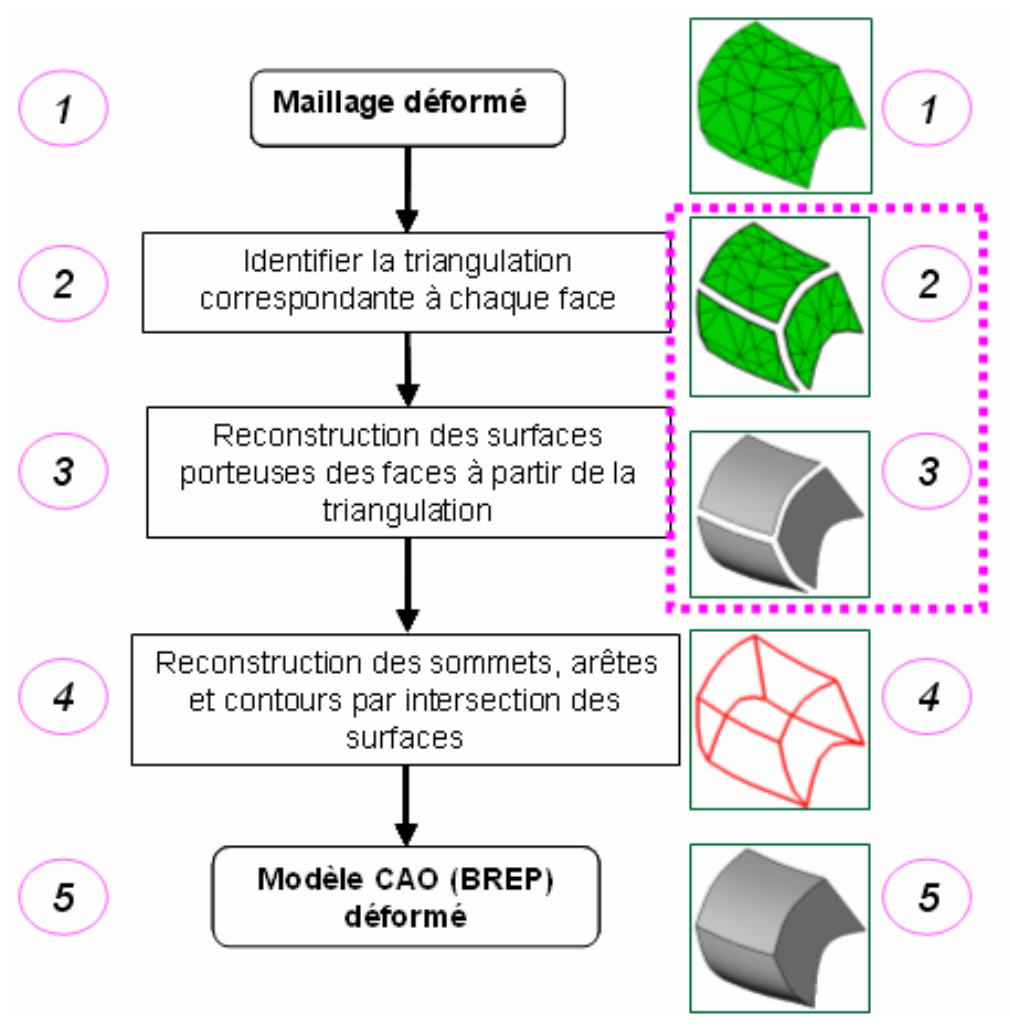

Figure 23 : Algorithme proposé

Dans l'étape 3, la reconstruction de la surface porteuse de la face déformée dépend directement des trois cas de figure introduits précédemment (cf. \$ 4.1).

Dans ce qui suit l'étape 3 sera développée en fonction de ces trois cas de figure (cf. \$ 4.1). 


\subsection{Reconstruction des Modèles déformés sans changement de géométrie et de topologie}

Cette reconstruction revient à reconstruire simplement les faces du modèle BRep. L'algorithme de reconstruction des faces est représenté par la figure 23.

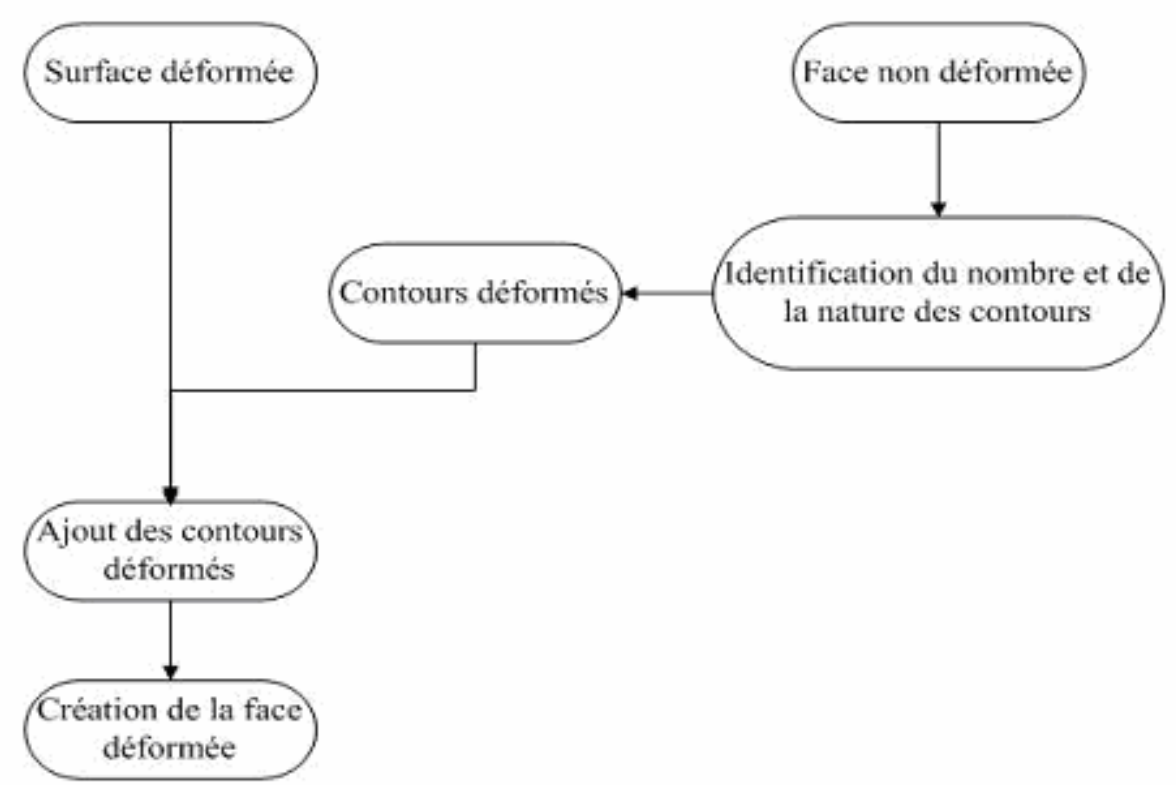

Figure 24 : Algorithme de reconstruction des faces

Dans cette reconstruction, il s'agit de déterminer les nouveaux paramètres de la face déformée, à savoir : le point d'insertion, le vecteur directeur, le rayon.... Par exemple, dans le cas d'une surface plane (figure ci-dessous), il s'agit de déterminer un point d'insertion (un nœud du maillage déformé lié à la face) et un vecteur normal au plan de la surface en utilisant les coordonnées de trois noeuds des arêtes déformés.

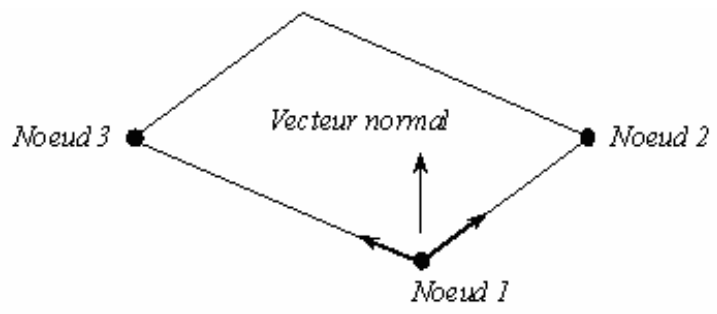

Figure 25 : Paramétrage d'une surface plane

\subsubsection{Exemple de validation.}

Pour valider l'algorithme de reconstruction dans le cas de petites perturbations, un exemple de système bielle manivelle a été étudié.

Dans l'état réel de fonctionnement, la bielle ainsi que le piston sont soumis à des chargements qui peuvent engendrer des déformations (figure 26). Sous COSMOS/M un calcul par éléments finis a permis de déterminer l'état de déformation de la bielle et du piston représenté par la figure suivante. Après application de l'algorithme détaillé ci-dessus, une reconstruction des faces et leurs connectivités a conduit aux modèles déformés de la bielle et du piston.

L'assemblage et la simulation du fonctionnement du mécanisme à l'état déformé ont permis de déceler des interférences et des collisions conduisant au disfonctionnement du mécanisme et à la remise en cause de la proposition de conception. 


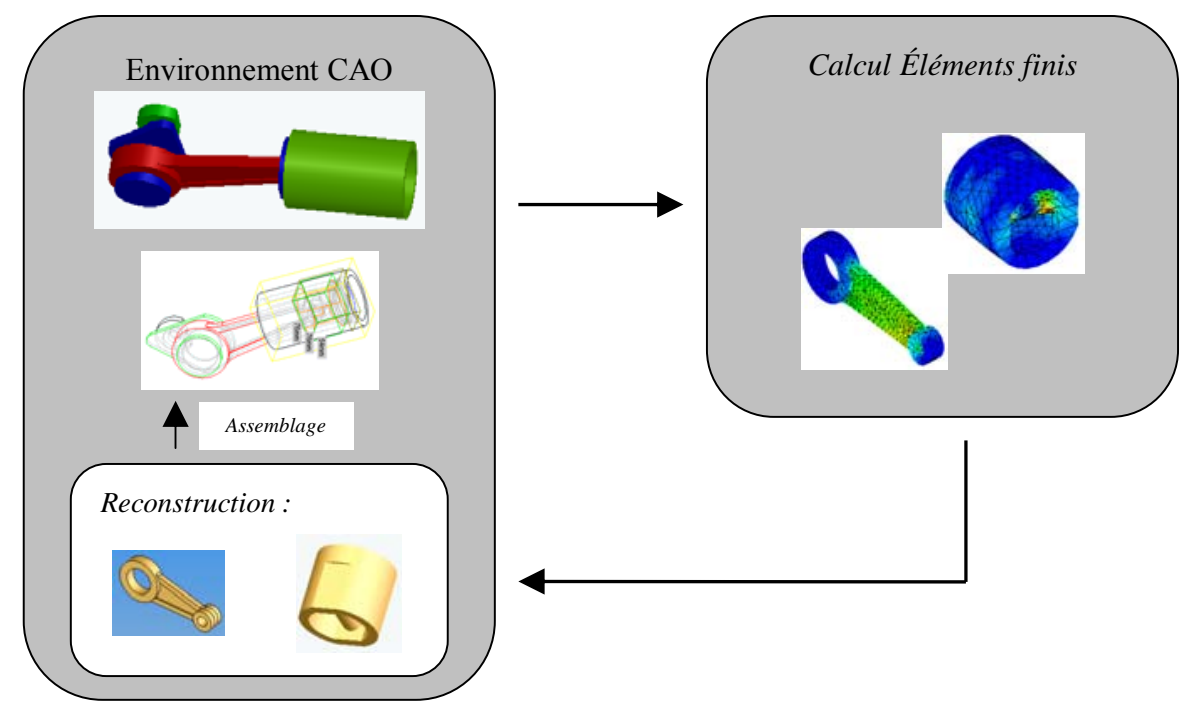

Figure 26 : Illustration de la démarche

\subsection{Reconstruction des modèles déformés avec changement de géométrie et sans changement de topologie.}

Dans ce cas de figure, on procède d'abord à une reconstruction des faces à partir du nuage de points récupéré du maillage déformé du solide considéré. Ces faces seront ensuite utilisées lors de la construction du BRep correspondant au solide déformé. L'algorithme de reconstruction proposé est représenté par la figure 27 ci-dessous.

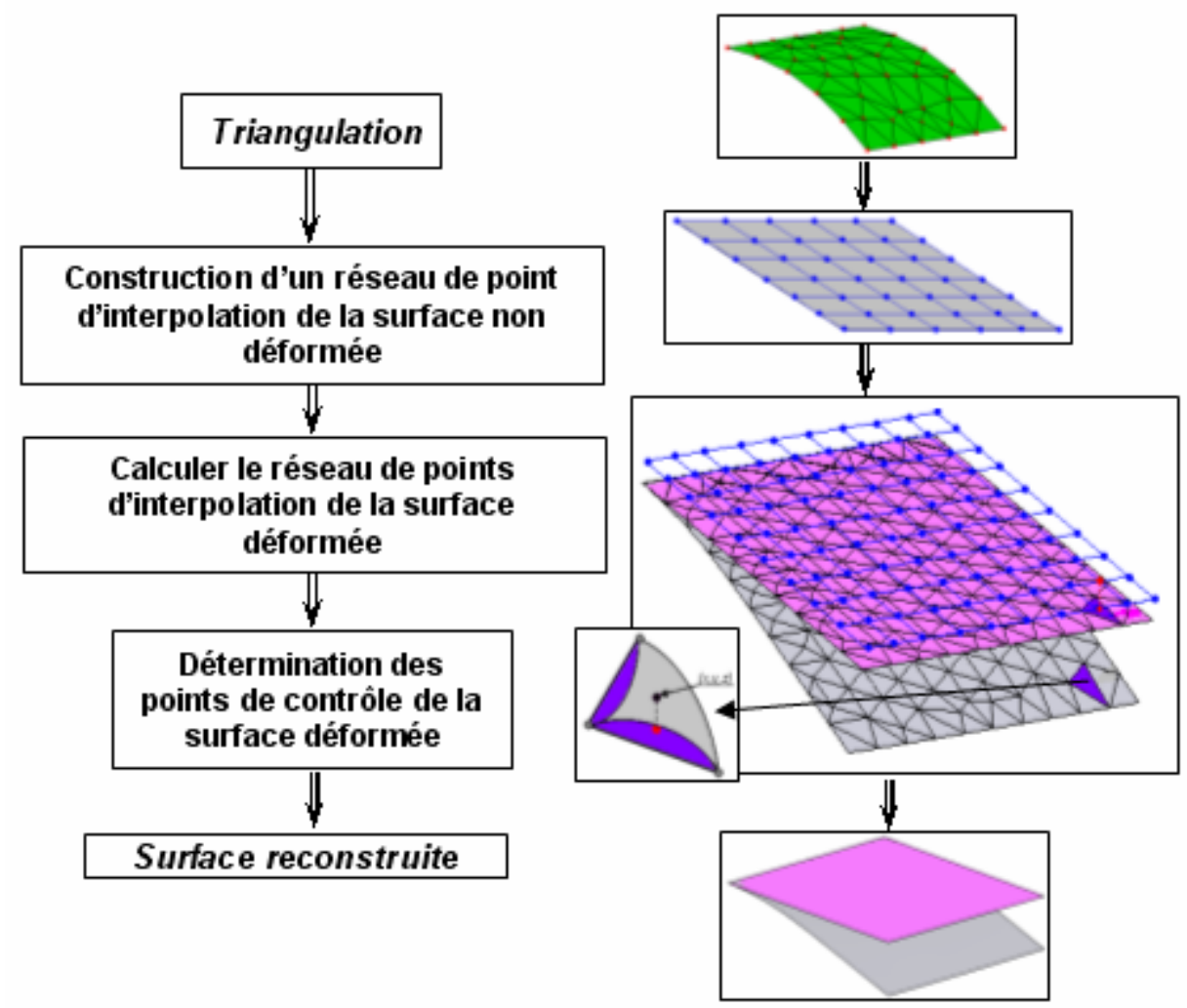

Figure 27 : Reconstruction d'une surface à partir d'une triangulation

Dans cet algorithme, il s'agit de déterminer les nouveaux paramètres de la surface porteuse de la face déformée, qui sont les points de contrôle comme illustré sur la figure ci-dessus. 


\subsubsection{Exemple de validation.}

Ce cas de figure peut être appliqué pour le cas des pièces en flexion (figure 28), Torsion, etc. Les deux exemples ci-dessous illustrent le cas de reconstruction du modèle CAO dans le cas ou la géométrie du modèle $\mathrm{CAO}$ change après déformation alors que la topologie du modèle initial est conservée.

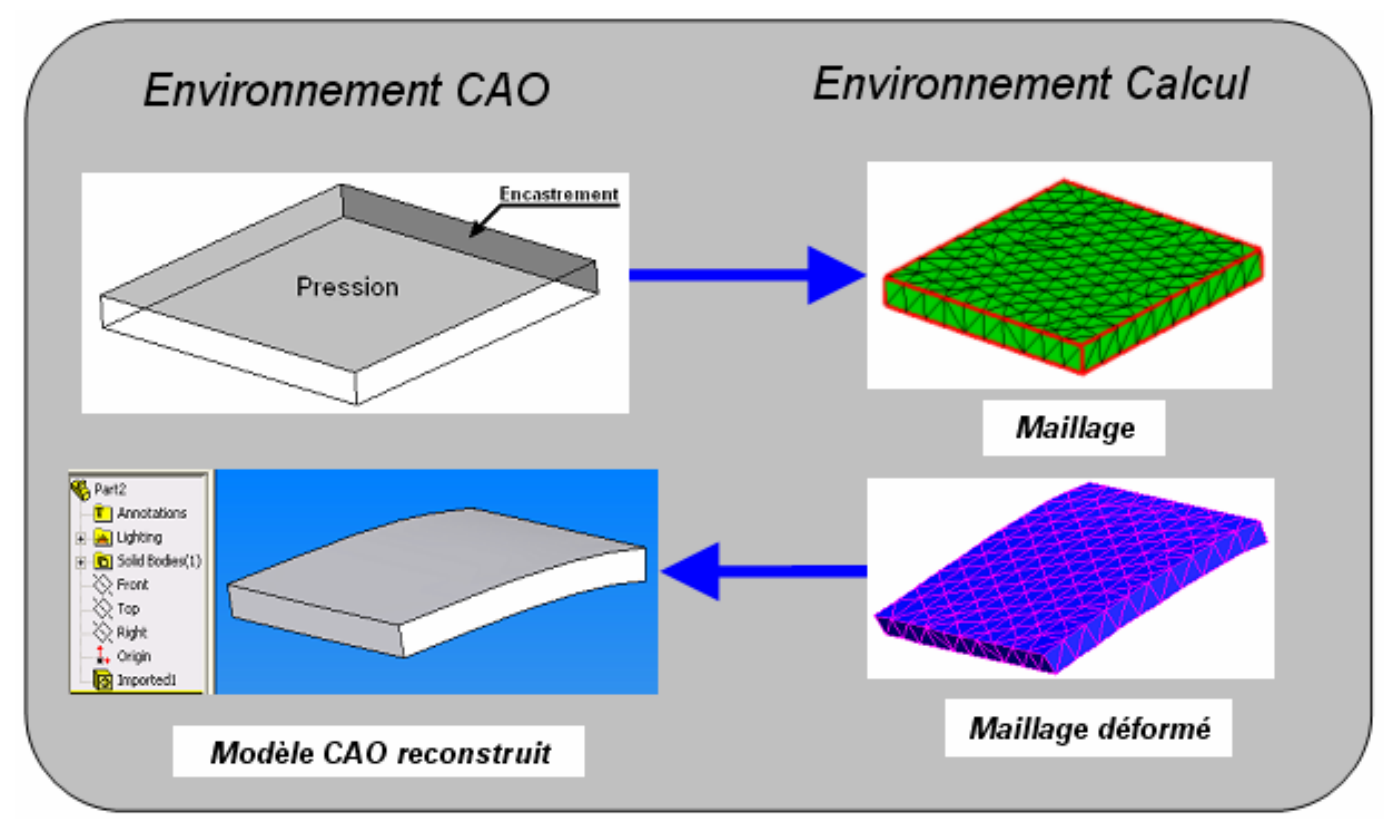

Figure 28 : Exemple d'illustration : Plaque en flexion.

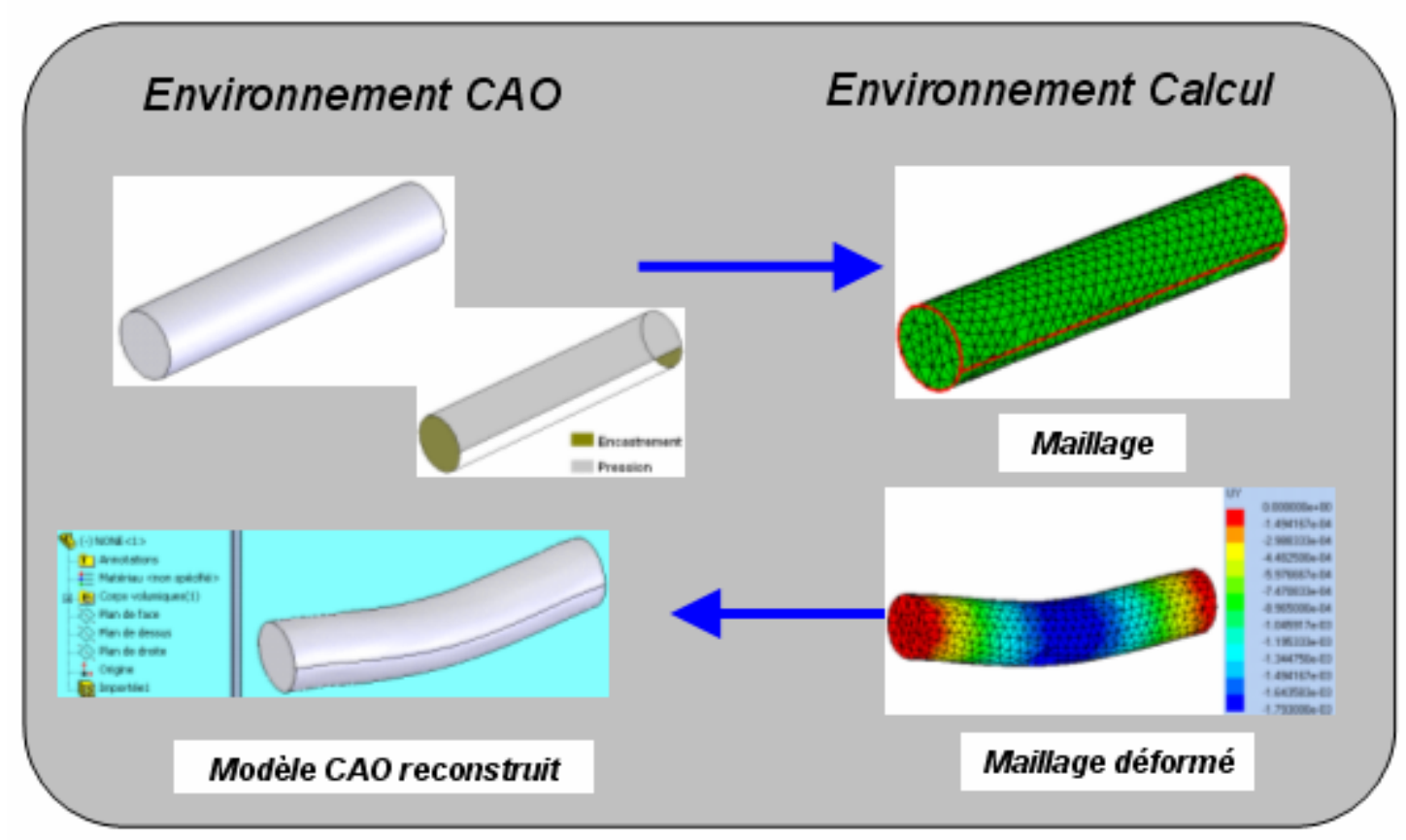

Figure 29 : Exemple d'illustration : Poutre en flexion.

\subsection{Reconstruction des modèles déformés avec changement de géométrie et changement de topologie.}

Ce cas de figure correspond essentiellement à la simulation des procédés de fabrication par déformation plastique qui consistent à partir d'une pièce mécanique ayant une géométrie et une topologie initiale et la transformer par déformation plastique en une nouvelle pièce mécanique ayant une forme géométrique et une topologie assez différente la pièce initiale (cas de l'emboutissage des 
tôles). Cela se traduit par un changement de la géométrie (forme et dimensions) et de la topologie (apparition des nouvelles faces, arêtes et sommets) (voir figure 30). La recherche de la nouvelle topologie (identification des nouvelles arêtes, sommets et faces) constitue une étape préalable avant d'entamer la reconstruction. Ce cas de figure est en cours d'étude dans le cadre de la thèse de Borhène Louhichi.

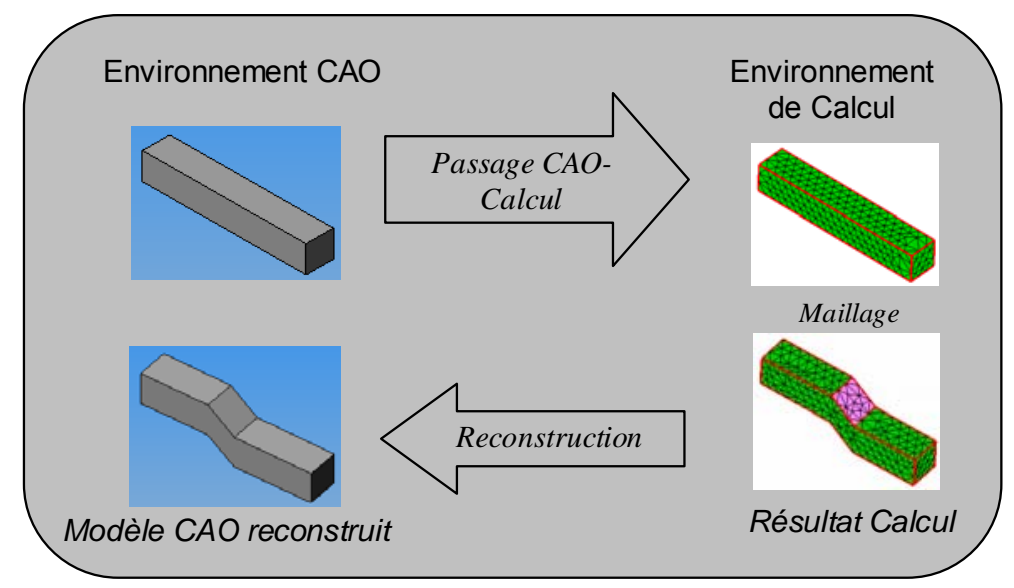

Figure 30 : Maillage déformé avec topologie inconnue

La simulation numérique des procédés de fabrication par transformation plastique peuvent également engendrer des maillages déformés dont il est difficile d'identifier la topologie (figure cidessous). Ce point fait également l'objet de nos investigations de recherche dans le cadre de la thèse de Borhène Louhichi.
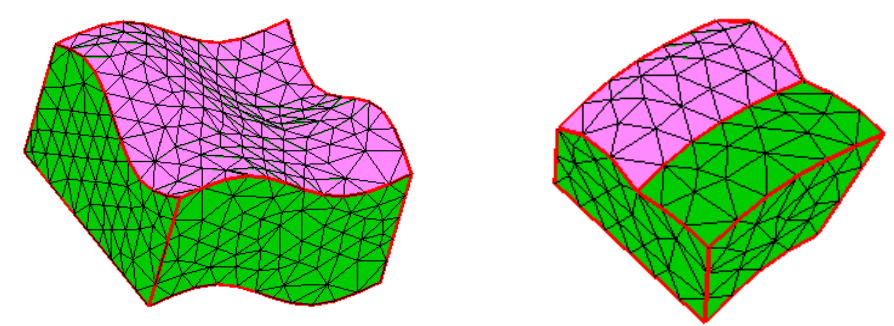

Figure 31 : Maillage déformé avec topologie inconnue

\subsection{Algorithmes de reconstruction d'une surface à partir d'un maillage surfacique.}

L'algorithme général proposé (Figure 27) pour la reconstruction d'un modèle CAO de type BREP à partir des résultats d'une analyse éléments finis (maillage déformé), fait appel (étape 3) à la reconstruction d'une surface à partir d'un maillage surfacique (maillage déformé des faces). Il s'agit donc de reconstruire une surface qui interpole le maximum de points de la triangulation (un nuage de points interconnectés par des segments).

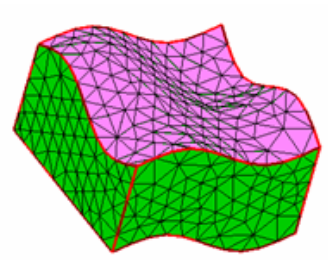

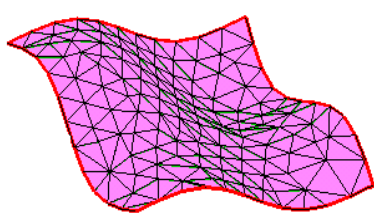

(a)

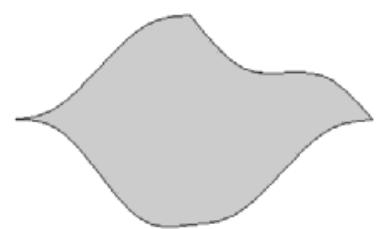

(b)

Figure 32 : Maillage déformé, Maillage surfacique(a), Surface d'interpolation du maillage (b).

En vue de trouver une méthode de reconstruction d'une surface à partir d'une triangulation, nous avons d'abord effectué une recherche bibliographique dont nous présentons ci-dessous un résumé des principales tendances. 


\subsubsection{Etat de l'art sur les méthodes de reconstruction de surfaces à partir d'un maillage.}

\subsubsection{Algorithme de Walton}

Walton [Walton 96] a proposé un algorithme d'évaluation d'une surface de Béziers à partir d'un triangle dans un maillage surfacique. Cet algorithme a été ensuite amélioré par Steven [Steven 01] [Steven 02].

L'algorithme consiste à calculer pour chacun des nœuds de la triangulation un vecteur. Ce vecteur sera ensuite utilisé comme la normale à la surface reconstruite (Figures 33, 34). Le calcul de ce vecteur normal se fait selon la formule suivante :

$$
\begin{aligned}
& N_{p}=\sum_{i=1}^{n} N_{i} * w_{i} \quad \text { Avec }: \\
& w_{i}=\frac{\alpha_{i}}{\sum_{i=1}^{n} \alpha_{i}}
\end{aligned}
$$

- $\boldsymbol{n}$ : est le nombre de triangles appartenant à la surface qui ont comme sommet le nœud $\boldsymbol{P}$.

- Les $\boldsymbol{N}_{\boldsymbol{i}}$ sont les normales aux triangles qui ont comme sommet le nœud $\boldsymbol{P}$.

- Les $\boldsymbol{w}_{\boldsymbol{i}}$ sont les coefficients de pondération associés aux normales $\boldsymbol{N}_{\boldsymbol{i}}$.

- Les $\alpha_{i}$ sont les angles au point $\boldsymbol{P}$ des triangles qui ont comme sommet le point $\boldsymbol{P}$.
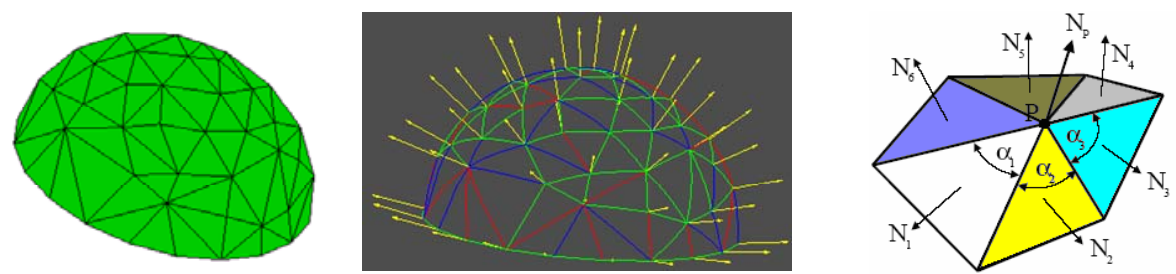

Figure 33 : Les normales à la surface aux noeuds aux nœuds.

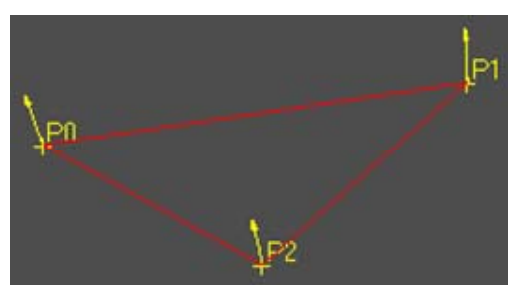

(a)

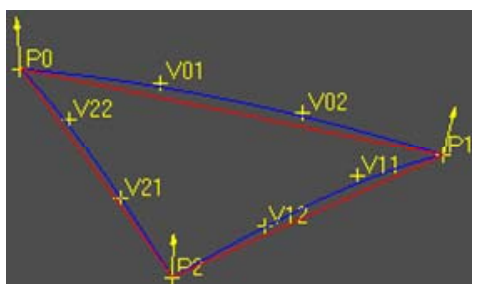

(b)

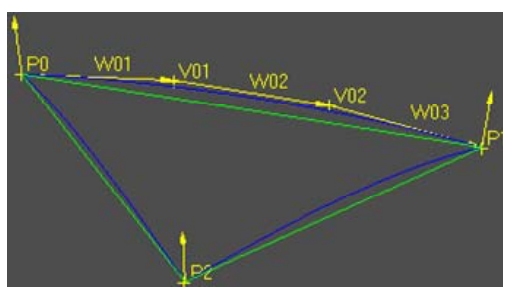

(c)

Figure 34 : (a) Les normales associées à un triangle, (b) Les courbes de frontières de la surface triangulaire, (c) Les tangentes aux courbes des frontières.

Par la suite, il s'agit d'évaluer les trois courbes frontières $\left(C_{i}(t)\right)$ (Fig. 20.) de la surface triangulaire :

$$
C_{i}(t)=\sum_{k=0}^{3} V_{i, k} B_{k, 3}(t) \quad \begin{aligned}
& 0 \leq t \leq 1 \\
& i=0,1,2
\end{aligned}
$$

Avec $\boldsymbol{B}_{i, n}$ est le polynôme de Bernstein défini par :

$$
\begin{array}{rl}
B_{i, n}=C_{n}^{i} t^{i}(1-t)^{n-i} & 0 \leq t \leq 1 \\
& i=0 \ldots, n
\end{array}
$$

Les $\boldsymbol{V}_{i, j}$ sont les points de contrôle des courbes frontières (Fig. 20.) et sont définies par les formules suivantes :

$$
V_{i, 0}=Q_{i} V_{i, 3}=Q_{i+1}
$$


Avec :

$$
V_{i, 1}=V_{i, 0}+d_{i} \cdot \frac{\left(6 \Gamma_{i}-2 \rho_{i} N_{i}+\sigma_{i} N_{i+1}\right)}{18} \text { Et } V_{i, 2}=V_{i, 3}-d_{i} \cdot \frac{\left(6 \Gamma_{i}+\rho_{i} N_{i}-2 \sigma_{i} N_{i+1}\right)}{18}
$$

$$
\begin{aligned}
& d_{i}=\left\|V_{i, 3}-V_{i, 0}\right\| \text { Est la distance }\left[\boldsymbol{P}_{\boldsymbol{i}} \boldsymbol{P}_{\boldsymbol{i}+1}\right] . \\
& \Gamma_{i}=\frac{\left(V_{i, 3}-V_{i, 0}\right)}{d_{i}} \text { Est le vecteur norme suivant la direction }\left(\boldsymbol{P}_{\boldsymbol{i}} \boldsymbol{P}_{\boldsymbol{i}+1}\right) . \\
& a_{i}=N_{i} \bullet N_{i+1} \text { Est le produit scalaire de deux vecteurs } \boldsymbol{N}_{\boldsymbol{i}} \text { et } \boldsymbol{N}_{\boldsymbol{i}+\mathbf{1}} \text {. } \\
& a_{i, 0}=N_{i} \bullet \Gamma_{i} \\
& a_{i, 1}=N_{i+1} \bullet \Gamma_{i} \\
& \rho_{i}=6 \frac{\left(2 a_{i, 0}+a_{i} a_{i, 1}\right)}{\left(4-a_{i}^{2}\right)} \\
& \text { Et } \quad \sigma_{i}=6 \frac{\left(2 a_{i, 1}+a_{i} a_{i, 0}\right)}{\left(4-a_{i}^{2}\right)} ; \quad \text { La tangente à } C_{\boldsymbol{i}}(\boldsymbol{t}) \text { est définie par : } \\
& \begin{array}{ccc} 
& i=0,1,2 & \text { Avec }: W_{i, k}=V_{i, k+1}-V_{i, k} \\
C_{i}{ }^{\prime}(t)=3 \sum_{k=0}^{2} W_{i, k} B_{k, 2}(t) & 0 \leq t \leq 1 & (k=0,1,2)
\end{array}
\end{aligned}
$$

Les points de contrôle de la surface triangulaire (triangle de Béziers) sont composés de deux groupes :

- Les points de contrôle sur les frontières qui sont définis directement à partir des points de contrôle des courbes frontières $\boldsymbol{V}_{\boldsymbol{i}, \boldsymbol{j}}$.

$$
P_{i, j}=\frac{1}{4}\left(j V_{i, j+1}+(4-j) V_{i, j}\right) \quad i=0,1,2 ; j=0 \ldots 4
$$

- Les points de contrôle en plein de la surface $\boldsymbol{P}_{1,1,2}, \boldsymbol{P}_{1,2,1}$ et $\boldsymbol{P}_{2,1,1}$ (Fig. 21.) et qui sont définies par les formules suivantes :

$$
\begin{aligned}
& P_{1,1,2}=\frac{1}{u+v}\left(u G_{2,2}+v G_{0,1}\right) \\
& P_{1,2,1}=\frac{1}{w+u}\left(w G_{0,2}+u G_{1,1}\right) e t \\
& P_{2,1,1}=\frac{1}{u+w}\left(u G_{1,2}+w G_{2,1}\right)
\end{aligned}
$$

Ces points $\left(\boldsymbol{P}_{1,1,2}, \boldsymbol{P}_{1,2,1}\right.$ et $\left.\boldsymbol{P}_{2,1,1}\right)$ dépendent des coordonnées paramétriques locales (coordonnées dans le triangle de Béziers) $\boldsymbol{u}, \boldsymbol{v}$ et $\boldsymbol{w}$.

Les vecteurs $G_{i, j}(i=0,1,2, j=1,2)$ sont calculés comme suit :

$$
\begin{aligned}
& G_{i, 1}=\frac{1}{2}\left(P_{i, 1}+P_{i, 2}\right)+\frac{2}{3} \lambda_{i, 0} W_{i, 1}+\frac{1}{3} \lambda_{i, 1} W_{i, 0}+\frac{2}{3} \mu_{i, 0} A_{i, 1}+\frac{1}{3} \mu_{i, 1} A_{i, 0} \\
& G_{i, 2}=\frac{1}{2}\left(P_{i, 2}+P_{i, 3}\right)+\frac{2}{3} \lambda_{i, 0} W_{i, 2}+\frac{1}{3} \lambda_{i, 1} W_{i, 1}+\frac{2}{3} \mu_{i, 0} A_{i, 2}+\frac{1}{3} \mu_{i, 1} A_{i, 1}
\end{aligned}
$$




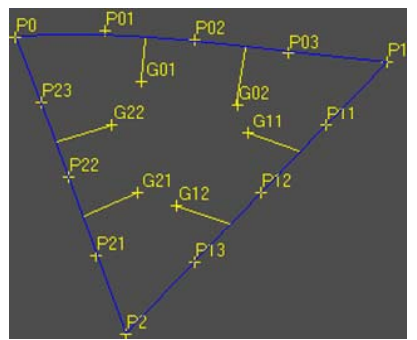

(a)

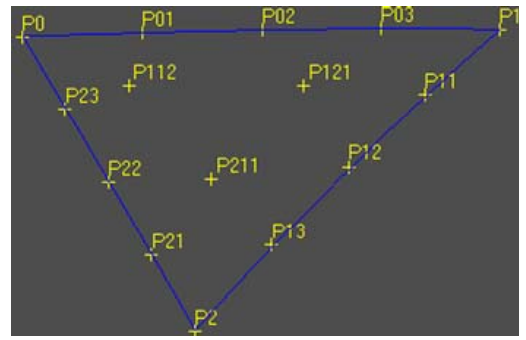

(b)

Figure 35 : Les points de contrôle de la surface (triangle de Béziers) $u=0.5, v=0.5$ et $w=0.5$.

Les vecteurs Gij sont définis pour assurer la continuité de la surface sur les frontières :

- Les vecteurs $\boldsymbol{G}_{\boldsymbol{0}}$ assurent la continuité sur la frontière définie entre $\boldsymbol{P}_{\mathbf{0}}$ et $\boldsymbol{P}_{\mathbf{1}}$.

- Les vecteurs $\boldsymbol{G}_{1 j}$ assurent la continuité sur la frontière définie entre $\boldsymbol{P}_{\mathbf{1}}$ et $\boldsymbol{P}_{\mathbf{2}}$.

- Les vecteurs $\boldsymbol{G}_{2 j}$ assurent la continuité sur la frontière définie entre $\boldsymbol{P}_{2}$ et $\boldsymbol{P}_{\mathbf{0}}$.

Pour cela les points de contrôle définis à l'intérieur de la surface $\boldsymbol{P}_{1,1,2}, \boldsymbol{P}_{1,2,1}$ et $\boldsymbol{P}_{2,1,1}$ sont définies par une combinaison de ces vecteurs $\boldsymbol{G}_{i j}$. Par exemple la définition de point $\boldsymbol{P}_{\mathbf{1 , 1 , 2}}$ doit garantir la continuité de la surface sur les deux frontières $\left(\boldsymbol{P}_{0}, \boldsymbol{P}_{1}\right)$ et $\left(\boldsymbol{P}_{2}, \boldsymbol{P}_{0}\right)$.

Ce qui fait : $P_{1,1,2}=\frac{1}{u+v}\left(u G_{2,2}+v G_{0,1}\right)$.

Les Coefficients $\lambda_{i, j}$ et $\mu_{i, j}(i=0,1,2, j=0,1)$ sont définie par :

$$
\begin{array}{lll}
\lambda_{i, 0}=\frac{D_{i, 0} \bullet W_{i, 0}}{\left(W_{i, 0} \bullet W_{i, 0}\right)} & A_{i, 0}=\frac{N_{i} \times W_{i, 0}}{\left\|W_{i, 0}\right\|} \\
\lambda_{i, 1}=\frac{D_{i, 3} \bullet W_{i, 2}}{\left(W_{i, 2} \bullet W_{i, 2}\right)} & \text { tel que, } A_{i j}: & A_{i, 2}=\frac{N_{i, 1} \times W_{i, 2}}{\left\|W_{i, 2}\right\|} \\
\mu_{i, 0}=D_{i, 0} \bullet A_{i, 0} & A_{i, 1}=\frac{A_{i, 0}+A_{i, 2}}{\left\|A_{i, 0}+A_{i, 2}\right\|} \\
\mu_{i, 1}=D_{i, 3} \bullet A_{i, 2} &
\end{array}
$$

Les $\boldsymbol{A}_{i j}$ sont les vecteurs qui définissent, avec les $\boldsymbol{W}_{i j}$, les plans tangents à la surface triangulaire de Béziers désirée afin d'assurer la continuité, de type $C^{1}$, de cette surface avec les surfaces voisins.

Les vecteurs $\boldsymbol{D}_{i, j}$ sont définies comme suit :

$$
\begin{aligned}
& D_{0, j}=P_{1, j, 3-j}-\frac{1}{2}\left(P_{0, j+1,3-j}+P_{0, j, 4-j}\right), \\
& D_{1, j}=P_{j, 3-j, 1}-\frac{1}{2}\left(P_{j+1,3-j, 0}+P_{j, 4-j, 0}\right) e t \\
& D_{2, j}=P_{3-j, 1, j}-\frac{1}{2}\left(P_{3-j, 0, j+1}+P_{4-j, 0, j}\right)
\end{aligned}
$$

Les $\boldsymbol{D}_{i j}$ sont les vecteurs qui assurent la continuité des plans tangents au triangle de Béziers désiré avec ses voisins, c'est-à-dire le dérivé de la surface à gauche et à droite de la courbe de frontière est la même.

On note :

$$
\boldsymbol{P}_{0, j}=\boldsymbol{P}_{0, j, 4-j}, \boldsymbol{P}_{1, j}=\boldsymbol{P}_{j, 4-j, 0} \text { et } \boldsymbol{P}_{2, j}=\boldsymbol{P}_{4-j, 0, j}
$$

La surface (triangle de Béziers), évaluer à partir d'un triangle, est définie par l'équation suivante : 


$$
\begin{aligned}
& S(u, v, w)=\sum_{i+j+k=4} P_{i, j, k} \frac{4 !}{i ! j ! k !} u^{i} v^{j} w^{k}, \\
& u, v, w \geq 0 ; \quad u+v+w=1 ; \quad i, j, k \geq 0
\end{aligned}
$$

Donc :

$$
\begin{gathered}
S(u, v, w)=P_{0} w^{4}+ \\
4 P_{23} u w^{3}+4 P_{01} v w^{3}+ \\
6 P_{22} u^{2} w^{2}+12 P_{112} u v w^{2}+6 P_{02} v^{2} w^{2}+ \\
4 P_{21} u^{3} w+12 P_{211} u^{2} v w+12 P_{121} u v^{2} w+4 P_{03} v^{3} w+ \\
P_{2} u^{4}+4 P_{13} u^{3} v+6 P_{12} u^{2} v^{2}+4 P_{11} u v^{3}+P_{1} v^{4}
\end{gathered}
$$

\subsubsection{Algorithme de REN}

Ren a développé [Ren 03] un algorithme de remaillage pour raffiner itérativement d'un maillage donné afin de se rapprocher de la forme réelle de l'objet maillé. Il s'agit d'insérer un nouveau nœud entre deux nœuds consécutifs de l'ancien maillage tout en respectant la nature de la surface. Cette subdivision consiste à diviser chaque triangle par quatre.

Étant donné $\boldsymbol{P}_{1}{ }^{l}$ et $\boldsymbol{P}_{2}{ }^{l}$ deux nœuds du maillage et $\boldsymbol{P}_{1, i}{ }^{l}$ et $\boldsymbol{P}_{2, i}{ }^{l}$ sont les nœuds qui sont autour de deux noeuds $\boldsymbol{P}_{1}{ }^{I}$ et $\boldsymbol{P}_{2}{ }^{I}$ respectivement (Figure ci-dessous), le nœud d'interpolation entre $\boldsymbol{P}_{1}{ }^{l}$ et $\boldsymbol{P}_{2}{ }^{l}$ est définie par:

$$
Q_{(l+1)}=\frac{1}{2}\left(\sum_{i=0}^{k_{1}-1} C_{1, i} P_{1, i}{ }^{l}+\sum_{j=0}^{k_{2}-1} C_{2, j} P_{2, j}{ }^{l}\right)
$$

Avec $\boldsymbol{P}_{1,0}{ }^{l}=\boldsymbol{P}_{2}{ }^{l}, \boldsymbol{P}_{2,0}{ }^{l}=\boldsymbol{P}_{1}{ }^{l}, \boldsymbol{C}_{1, i}$ et $\boldsymbol{C}_{2, j}$ sont les coefficients poids correspond à $\boldsymbol{P}_{1}{ }^{l}$ et $\boldsymbol{P}_{2}{ }^{l}$ (Fig. 23.).
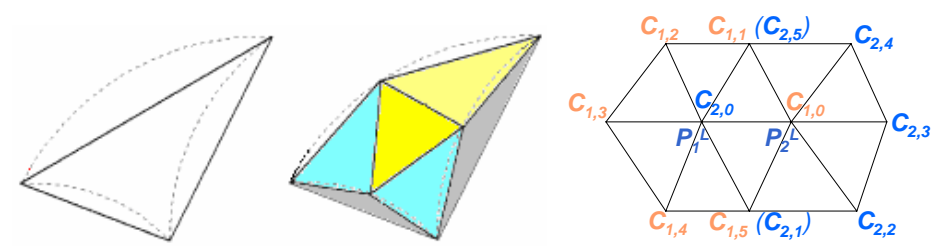

Figure 36 : Subdivision d'un maillage et coefficients attribués à chacun des points voisins [Ren 03].

Les $\mathrm{C}_{\mathrm{m}, \mathrm{i}}$ sont définis par :

$$
\left\{\begin{array}{l}
C_{m, i}=\frac{1}{k}\left(\frac{1}{4}+\cos \frac{2 i \pi}{k}+\frac{1}{2} \cos \frac{4 i \pi}{k}\right) \quad(m=1,2 ; i=1, \ldots, k-1) \\
C_{m, 0}=1-\sum_{i=1}^{k-1} C_{m, i}
\end{array}\right.
$$

Ren [Ren 03] a également développé un algorithme pour la modélisation des contours en se basant sur des courbes de continuité $\boldsymbol{C}^{\boldsymbol{1}}$ pour la modélisation des frontières des surfaces. Il s'agit d'insérer deux points virtuels à chaque itération, l'algorithme est le suivant :

$$
\begin{cases}P_{2 i}{ }^{(k+1)}=P_{i}{ }^{(k)} & \left(0 \leq i \leq 2^{k} n-1\right) \\ P_{2 i+1}{ }^{(k+1)}=\left(\frac{1}{2}+w\right) \cdot\left(P_{i}^{(k)}+P_{i+1}{ }^{(k)}\right)-w \cdot\left(P_{i-1}{ }^{(k)}+P_{i+2}{ }^{(k)}\right) & \left(0 \leq i \leq 2^{k} n-1\right)\end{cases}
$$

Avec les $\boldsymbol{P}_{i}^{k}$ sont les nœuds des frontières et $0<w<(\sqrt{5}-1) / 8$ généralement $w=1 / 16$. 


$$
\begin{cases}P_{2 i}{ }^{(k+1)}=P_{i}^{(k)} & \left(0 \leq i \leq 2^{k} n-1\right) \\ P_{2 i+1}{ }^{(k+1)}=\frac{1}{2} \cdot\left(P_{i}^{(k)}+P_{i+1}{ }^{(k)}\right)-2 \cdot w \cdot Q_{i}{ }^{(k)} & \left(0 \leq i \leq 2^{k} n-1\right)\end{cases}
$$

Avec :

$$
Q i(k)=\frac{1}{2} \cdot\left(P_{i}^{(k)}+P_{i+1}{ }^{(k)}\right)-\frac{1}{2} \cdot\left(P_{i-1}{ }^{(k)}+P_{i+2}{ }^{(k)}\right)
$$

L'algorithme de Ren [Ren 03] fait appel à quatre points du maillage initial à chaque itération pour avoir une courbe de continuité $\boldsymbol{C}^{1}$ et six points pour avoir une courbe de continuité $\boldsymbol{C}^{2}$.

$$
\left\{\begin{array}{cc}
P_{2 i}{ }^{(k+1)}=P_{i}^{(k)} & \left(0 \leq i \leq 2^{k} n-1\right) \\
P_{2 i+1}{ }^{(k+1)}=\left(\frac{9}{16}+2 \cdot \alpha\right) \cdot\left(P_{i}^{(k)}+P_{i+1}{ }^{(k)}\right)-\alpha \cdot\left(P_{i-2}{ }^{(k)}+P_{i+3}{ }^{(k)}\right)-\left(\frac{1}{16}+3 \cdot \alpha\right) \cdot\left(P_{i-1}{ }^{(k)}+P_{i+2}{ }^{(k)}\right) & 0 \leq \alpha \leq 0.02
\end{array}\right.
$$

Avec les $\boldsymbol{P}_{i}{ }^{k}$ sont les nœuds des frontières $0<w<(\sqrt{5}-1) / 8$

\subsubsection{Algorithme de Volpin}

Volpin [Volpin 98] a proposé un algorithme de reconstruction d'une surface NURBS à partir d'un maillage en passant par une simplification de ce dernier.

La méthode de Volpin commence par la simplification du modèle maillé initial à travers la construction des régions restreintes en courbures conformément au modèle. Par la suite il construit un maillage quadrilatéral du modèle et enfin il construit des surfaces lisses à ce maillage en utilisant une méthode appelée méthode d'énergie (Figure 37).

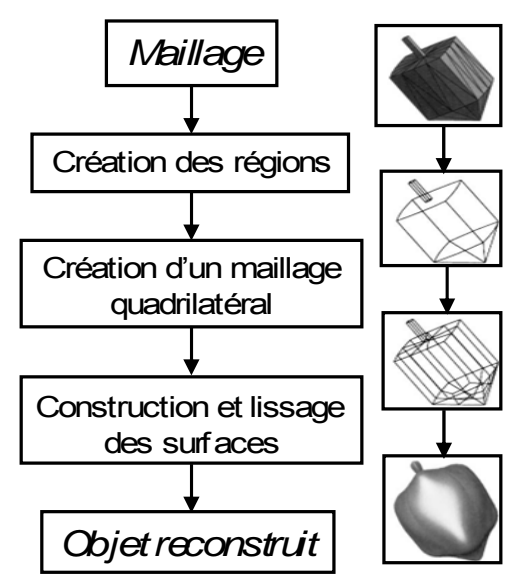

Figure 37 : Simplification du maillage et construction des surfaces.

Pendant la première phase, simplification du modèle, Volpin se base sur des critères pour la création des régions restreintes en courbure qui sont l'angle minimal entre deux facettes de maillage pour qu'elles soient dans la même région et l'angle pour la création des arêtes entre les régions. Après le maillage quadratique, Volpin assure la continuité $C^{1}$ entre les surfaces reconstruites à partir des régions.

Les régions restreintes sont de types planes, les plans correspondants sont définis par les formules suivantes : $N_{x} x+N_{y} y+N_{z} z+d=0$ 
La normale est définis par : $N_{c}=\frac{1}{m \times \operatorname{air}(c)} \sum_{i=1}^{m} \operatorname{air}\left(f_{i}\right) N_{i}$

La distance d est définie par : $d_{c}=\frac{1}{m \times \operatorname{air}(c)} \sum_{i=1}^{m} \operatorname{air}\left(f_{i}\right) d_{i}$

La création de régions se fait selon trois critères :

- La distance entre une facette du maillage (maille) et un plan correspondant à une région.

- L'angle entre deux facettes adjacentes du maillage.

- L'angle entre un plan correspondant à une région construite et une facette.

\subsubsection{Autres travaux}

D'autres recherches sont faites sur la reconstruction d'une surface approximative à partir d'un nuage de points en s'appuient sur les frontières. Kruth [Kruth 98] et Zhongwei [Zhongwei 04] ont développé une méthode de reconstruction d'une surface à partir d'un nuage de points (dans la plus part des cas régulier) issu d'une machine MMT (Figure 38). Dans le même contexte Piegl [Piegl 01] a développé une méthode de paramétrage et lissage d'une surface à partir de quatre courbes de frontières et un ensemble ordonné de points.

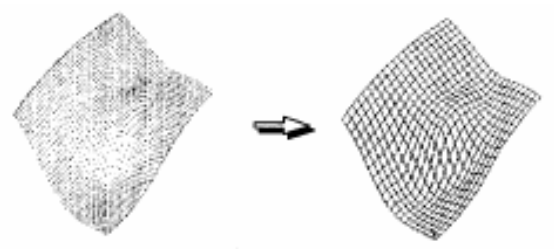

Figure 38 : Reconstruction d'une surface NURBS [Kruth 98].

\subsubsection{Algorithme proposé pour la reconstruction d'une surface à partir d'une triangulation.}

Les méthodes présentées dans le paragraphe précédant nous ont permis de procéder, à la construction d'une surface à partir d'une triangulation, selon deux approches distinctes :

\subsubsection{Approche (A)}

Cette approche consiste à construire un réseau de points réguliers, qui est un support d'interpolation pour la surface porteuse de la face déformée (dans la plus part des cas est une surface libre de type NURBS [Piegl 97] [Jung 00]). Nous nous sommes limités au cas du maillage tétraédrique c'est-à-dire triangulaire sur les faces et par conséquent cela correspond à un nuage de points irrégulièrement disposés liés les uns aux autres par des segments pour former des triangles. Or d'après la théorie des surfaces libres (surfaces NURBS) il faut que ce réseau de points soit régulièrement reparti. Donc le problème revient à construire, d'abord, un support de points réguliers à partir d'une triangulation quelconque.

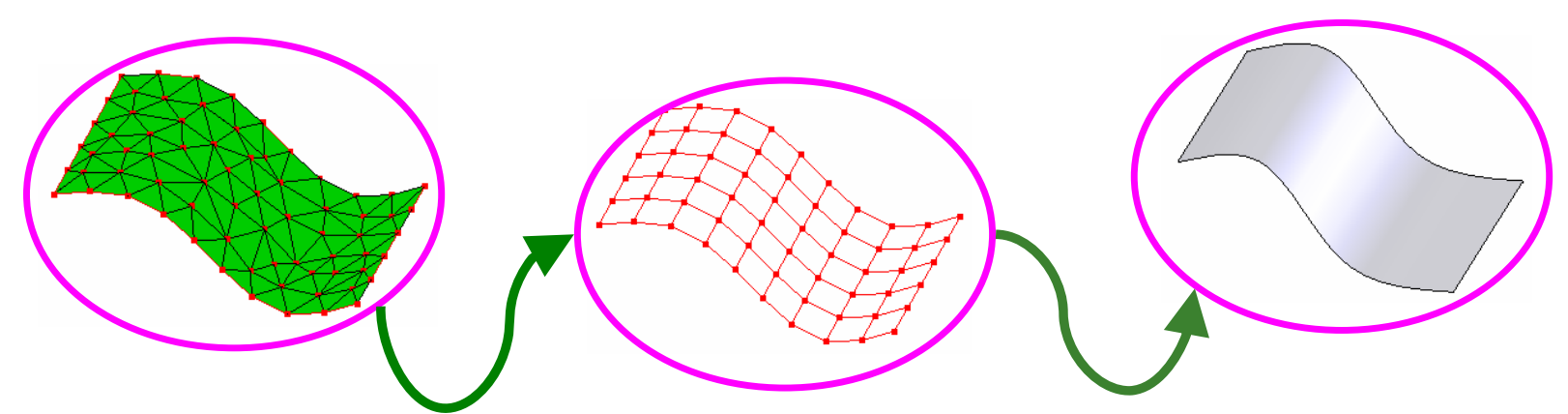

Figure 39 : (a) Triangulation, (b) Support régulier de points. 
L'algorithme proposé est basé sur le paramétrage de la surface avant déformation et la solution éléments finis (maillage déformé + les déplacements des noeuds). Il s'agit de construire un support de points réguliers par interpolation de la solution éléments finis sur un support régulier de points (figure 39).

Les étapes de l'algorithme sont les suivants :

- Construire un réseau de points réguliers sur la face non déformé.

- Projeter ce support de points sur le maillage non déformé.

- Voir pour chacun des points le triangle d'appartenance de sa projection et les coordonnées locales de ces points dans ce triangle.

- Déterminer les coordonnées de point dans le support déformé en se basant sur les coordonnées initiales avant déformation, les coordonnées locales du point dans le triangle et les déplacements des nœuds (la solution éléments finis).

Il s'agit de créer un réseau de points dans l'espace paramétrique (Figure 40.) de coordonnées $(u, v)$ qui corresponde à un réseau de points dans l'espace réel $(x, y, z)$.

Les cordonnées dans l'espace paramétrique sont déterminés comme suit :

$$
\begin{aligned}
& u_{i}=u_{i-1}+\frac{u_{\text {max }}-u_{\min }}{n} \\
& v_{j}=v_{j-1}+\frac{v_{\text {max }}-v_{\min }}{m}
\end{aligned}
$$

- $u_{\min }, u_{\max }, v_{\min }, v_{\max }$ : sont déterminés à partir des coordonées paramétriques des nœuds du maillage issues du modele intégré qui contient les informations sur les différents noeuds.

- $n$ et $m$ : sont les nombres des points suivant l'axes $\boldsymbol{u}$ et $\boldsymbol{v}$ dans l'éspace paramétrique.

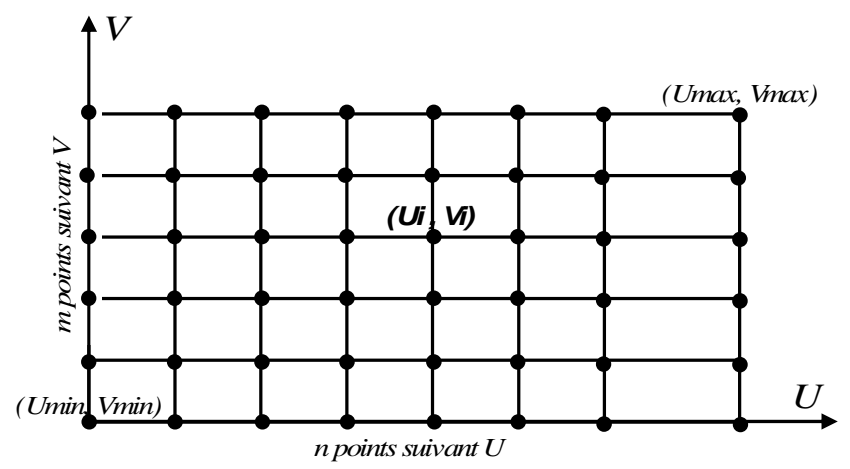

Figure 40 : Support de points dans l'espace paramétrique.

Pour le moment, le choix de nombre de points ( $n$ et $m$ ) suivant $\boldsymbol{u}$ et $\boldsymbol{v}$ se fait manuellement. Lorsque la méthode sera opérationnelle, elle possèdera un algorithme permettant d'optimiser les valeurs de $n$ et $m$.

La deuxième étape consiste à déterminer le support de points dans l'espace réel $(\mathrm{x}, \mathrm{y}, \mathrm{z})$ correspondant au réseau crée dans la première étape. Pour ce faire, on a recours à l'algorithme d'évaluation précité [Pieg1 97].

Rappelons que l'algorithme d'évaluation [Piegl 97] permet de calculer les coordonnées $x, y$ et $z$ en lui donnant les coordonnées paramétriques $\boldsymbol{u}$ et $\boldsymbol{v}$.

A la fin de cette étape, on obtient un réseau de points réguliers (Figure 41, 42) dans l'espace réel de la surface $(0, x, y, z)$. 


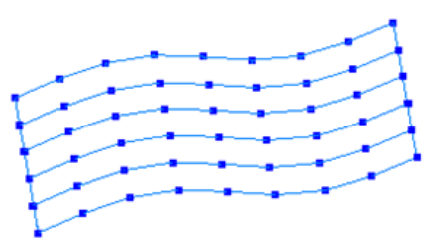

(a)

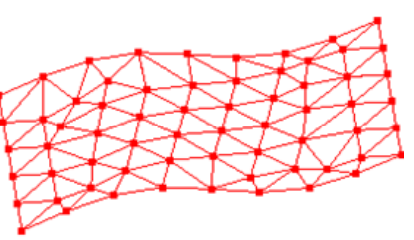

(b)

Figure 41 : (a) Réseau de points, (b) Maillage surfacique.

L'étape 3 de l'algorithme consiste à interpoler la solution éléments finis de type déplacement sur le nuage de points réguliers construit dans l'étape 2 .

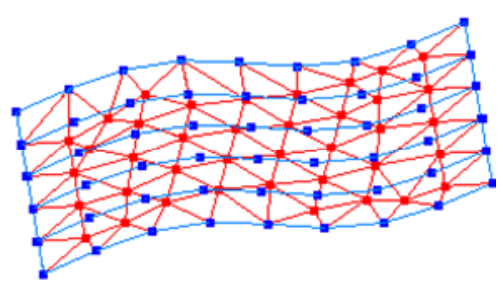

(a)

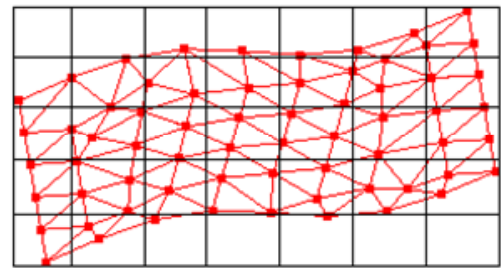

(b)

Figure 42 : Réseau des points réguliers et maillage, (b) Maillage placé dans une grille.

Les coordonnées de référence du nœud sont déterminées par projection de chacun des points sur un triangle correspondant du maillage (Figure ci-dessus).

Après l'obtention du support régulier des points de la surface déformée qui sont les points d'interpolation, l'étape 4 de l'algorithme consiste à déterminer les points de contrôle $\boldsymbol{P}_{i, j}$ de la surface déformée à partir des points d'interpolation $\boldsymbol{Q}_{\boldsymbol{k}, \boldsymbol{l}}$ par résolution du système inverse de l'équation suivante [Jung 00]:

$Q_{k, l}=\sum_{i=0}^{n} \sum_{j=0}^{m} N_{i, p}\left(\bar{u}_{k}\right) N_{j, q}\left(\bar{v}_{l}\right) P_{i, j}$

$N_{i, p}(u)$ Sont les fonction d'interpolation ou fonction de base [Pieg1 97] définies par :

$$
N_{i, p}(u)=\frac{u-u_{i}}{u_{i+p}-u_{i}} N_{i, p-1}(u)+\frac{u_{i+p+1}-u}{u_{i+p+1}-u_{i+1}} N_{i+1, p-1}(u) \quad N_{i, 0}(u)= \begin{cases}1 & \text { si } u_{i} \leq u \leq u_{i+1} \\ 0 & \text { sinon }\end{cases}
$$

$u_{i}$ les valeurs du vecteur nœuds.

$$
\begin{gathered}
Q_{k, l}=\sum_{i=0}^{n} N_{i, p}\left(\overline{u_{k}}\right)\left(\sum_{j=0}^{m} N_{j, q}\left(\overline{v_{l}}\right) P_{i, j}\right) ; \\
Q=N_{u} \times\left(N_{v} \times P\right) \\
Q \text { : la matrice des points d'interpolation. } \\
P \text { : la matrice des points de contrôle. } \\
N_{u} \text { : la matrice des fonctions de base suivant } \mathrm{u} . \\
N_{v} \text { : la matrice des fonctions de base suivant } \mathrm{v} . \\
Q=N_{u} \times R ; R=N_{v} \times P \text {; Donc } R=N_{u}{ }^{-1} \times Q \text { Et } P=N_{v}{ }^{-1} \times R
\end{gathered}
$$

En écriture indicielle :

$$
Q_{k, l}=\sum_{i=0}^{n} N_{i, p}\left(\bar{u}_{k}\right) R_{i, l} \quad\left\{\begin{array}{l}
k=0, . ., n \\
m=0, . ., m
\end{array}\right.
$$

Les valeurs des paramètres $\boldsymbol{u}_{\boldsymbol{i}}$ sont définies par la méthode suivante : 


$$
\bar{u}_{0}=0, \quad \bar{u}_{n}=1 \quad \bar{u}_{i}=\bar{u}_{i-1}+\frac{\left|Q_{i}-Q_{i-1}\right|^{\frac{1}{2}}}{\sum_{j}^{m}\left|Q_{j}-Q_{j-1}\right|^{\frac{1}{2}}}
$$

Pour un choix de degré (exemple degré $=3$ ) Le système suivant permet de calculer les $\boldsymbol{R}_{\boldsymbol{i}, \boldsymbol{I}}$

$$
\begin{gathered}
{\left[\begin{array}{cccc}
N_{0,3}\left(\bar{u}_{0}\right) & N_{1,3}\left(\bar{u}_{0}\right) & \ldots & N_{n, 3}\left(\bar{u}_{0}\right) \\
N_{0,3}\left(\bar{u}_{1}\right) & N_{1,3}\left(\bar{u}_{1}\right) & \ldots & \ldots \\
\ldots & \ldots & \ldots & \ldots \\
\ldots & \ldots & \ldots & \ldots \\
N_{0,3}\left(\bar{u}_{n}\right) & N_{1,3}\left(\bar{u}_{n}\right) & \ldots & N_{n, 3}\left(\bar{u}_{n}\right)
\end{array}\right]\left[\begin{array}{cccc}
R_{0,0} & R_{0,1} & \ldots & R_{0, m} \\
R_{1,0} & R_{1,1} & \ldots & \ldots \\
\ldots & \ldots & \ldots & \ldots \\
\ldots & \ldots & \ldots & \ldots \\
R_{n, 0} & \ldots & \ldots & R
\end{array}\right]=\left[\begin{array}{cccc}
Q_{0,0} & Q_{0,1} & \ldots & Q_{0, m} \\
Q_{1,0} & Q_{1,1} & \ldots & \ldots \\
\ldots & \ldots & \ldots & \ldots \\
\ldots & \ldots & \ldots & \ldots \\
Q_{n, 0} & \ldots & \ldots & Q_{n, m}
\end{array}\right]} \\
N_{u} R=Q \text { Donc } R=N_{u}{ }^{-1} Q
\end{gathered}
$$

Et on a : $R_{i, l}=\sum_{j=0}^{m} N_{j, q}\left(v_{l}\right) P_{i, j}$ avec $\left\{\begin{array}{l}i=0, \ldots, n \\ l=0, \ldots, m\end{array}\right.$

$$
\begin{gathered}
{\left[\begin{array}{cccc}
N_{0,3}\left(\bar{v}_{0}\right) & N_{1,3}\left(\bar{v}_{0}\right) & \ldots & N_{m, 3}\left(\bar{v}_{0}\right) \\
N_{0,3}\left(\bar{v}_{1}\right) & N_{1,3}\left(\bar{v}_{1}\right) & \ldots & \ldots \\
\ldots & \ldots & \ldots & \ldots \\
\ldots & \ldots & \ldots & \ldots \\
N_{0,3}\left(\bar{v}_{m}\right) & \ldots & \ldots & N_{m, 3}\left(\bar{v}_{m}\right)
\end{array}\right]\left[\begin{array}{cccc}
P_{0,0} & P_{1,0} & \ldots & P_{n, 0} \\
P_{0,1} & P_{1,1} & \ldots & \ldots \\
\ldots & \ldots & \ldots & \ldots \\
\ldots & \ldots & \ldots & \ldots \\
P_{0, m} & \ldots & \ldots & P_{n, m}
\end{array}\right]=\left[\begin{array}{cccc}
R_{0,0} & R_{1,0} & \ldots & R_{n, 0} \\
R_{0,1} & R_{1,1} & \ldots & \ldots \\
\ldots & \ldots & \ldots & \ldots \\
\ldots & \ldots & \ldots & \ldots \\
R_{0, m} & \ldots & \ldots & R_{n, m}
\end{array}\right]} \\
N_{v} P=R \text { Donc } P=N_{v}^{-1} R
\end{gathered}
$$

$\boldsymbol{P}$ est la matrice des $\boldsymbol{P}_{i, j}$ qui sont les points de contrôles de la surface porteuse de la face déformée.

\subsubsection{Approche (B)}

Lors des tests de validation de l'approche A, les limitations suivantes ont été constatées :

- Les points d'interpolation sont situés sur le maillage déformé (qui ne présente pas, dans la plus part des cas, la surface).

- L'oscillation de la surface qui vient aussi du fait que les points d'interpolation sont situés sur le maillage (lors de l'interpolation, la surface passe de coté et de l'autre du maillage). Pour cela la surface doit être calculée, au moins localement, au voisinage du maillage lors de l'ajout d'un point d'interpolation, pour que ce point ne soit pas sur le maillage par contre sur la surface qui doit être reconstruite.

L'approche $\mathrm{B}$ est donc une amélioration de l'approche A qui consiste à tirer profit de la méthode de Walton pour pallier aux problèmes de précision constatés lors des tests de l'approche A.

Au niveau de la troisième étape de l'algorithme on fait appel à l'algorithme de Walton [Walton 96] qui consiste à évaluer la surface localement à partir de la triangulation.

L'algorithme de Walton [Walton 96], consiste à évaluer la surface localement pour chaque triangle du maillage selon les étapes suivantes (Figure 42) :

- Calculer les coordonnées locales du point (support non déformé) projeté dans le triangle (maillage non déformé) $\boldsymbol{u}, \boldsymbol{v}$ et $\boldsymbol{w}$,

- Insérer un point qui a comme coordonnées $\boldsymbol{u}, \boldsymbol{v}$ et $\boldsymbol{w}$ dans le même triangle de maillage déformé, 
- A partir du point inséré précédemment, déterminer le point de support d'interpolation déformé qui doit être sur la surface qui va être reconstruite (Algorithme de Walton [Walton 96]).

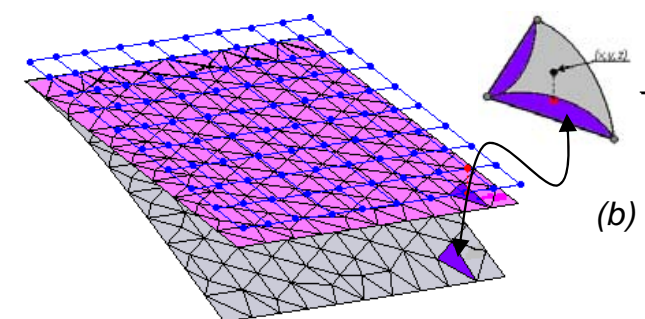

(a)

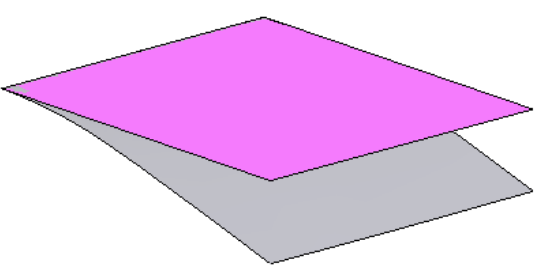

(c)

Figure 43 : Procédure d'évaluation et de reconstruction d'une surface.(a) Maillage déformé et non déformé, (b) Surface évaluée à partir d’un triangle, (c) Surface reconstruite.

Par exemple, pour la face plane reconstruite (Figure 43.), l'ordre de dimensions de la face est 105 $\mathrm{mm}$, les déplacements des nœuds sont de l'ordre de $104 \mathrm{~mm}$. Les erreurs des nœuds de maillage par rapport à la surface reconstruite sont dans le tableau 3 :

\begin{tabular}{|c|c|c|c|c|c|}
\hline $\begin{array}{c}\text { Nombre de } \\
\text { nœuds }\end{array}$ & $\begin{array}{c}\text { Nombre de } \\
\text { points } \\
\text { d'interpolation }\end{array}$ & Erreur Max & Erreur Min & $\begin{array}{c}\text { Moyenne des } \\
\text { erreurs }\end{array}$ & Écart Type \\
\hline 139 & 121 & $0,11 \mathrm{~mm}$ & $3,910^{-6} \mathrm{~mm}$ & $1,410^{-2} \mathrm{~mm}$ & $2,210^{-2}$ \\
\hline
\end{tabular}

Tableau 3 : Les erreurs aux nouds par rapport à la surface reconstruite (cas de surface plane).

On peut conclure que les erreurs sont négligeables par rapport aux dimensions de la surface pour cet exemple puisque les grandeurs de dimensions de la face sont de l'ordre de $105 \mathrm{~mm}$ et les déplacements sont aussi de l'ordre de $104 \mathrm{~mm}$.

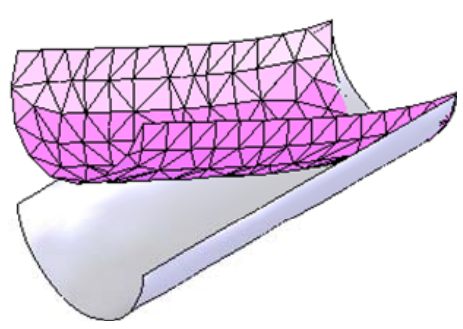

(a)

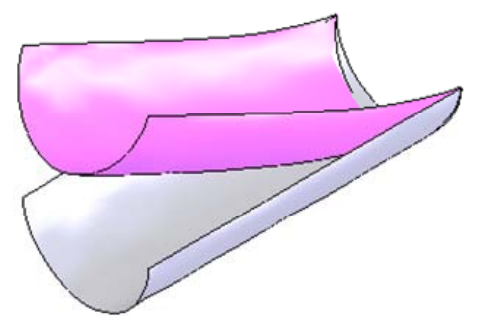

(b)

Figure 44 : (a) Maillage d’une face cylindrique déformée, (b) Face cylindrique déformée.

Des tests ont été faits sur des faces sans déformation afin de s'assurer de la performance de l'approche (Figure 44).

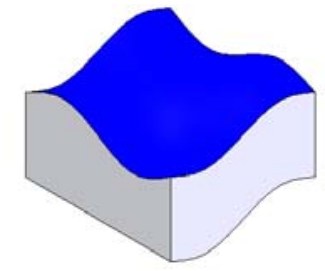

(a)

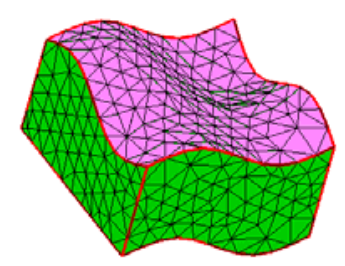

(b)

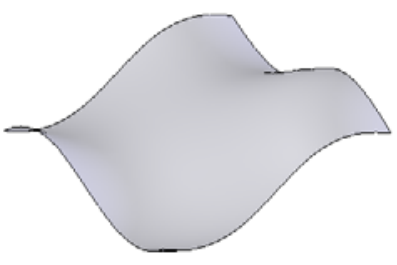

(c)

Figure 45 : (a) Face (Modèle CAO initial), (b) Maillage de la face, (c) Face reconstruite.

Comparées aux dimensions des pièces considérées, les erreurs restent raisonnables dans les cas des deux tests précédants. 


\subsubsection{Limites de l'algorithme proposé.}

Les premiers résultats sont obtenus par l'application de cette approche sur des pièces de topologie simple et qui sont soumis à des chargements de type simple, nous avons ensuite fait des tests avec des pièces de géométrie complexe et nous avons constaté les limitations suivantes :

- La méthode tient compte de la topologie initiale (chaque face est reconstruite en se basant sur le maillage initial), donc peu utile pour le cas des grandes déformations induisant des changement topologiques sur le modèle initial.

- L'approche est limitée, jusqu'à maintenant, aux surfaces sans contours intérieurs.

\subsection{Synthèse sur les approches proposées.}

En vue de reconstruire le modèle CAO déformé à partir d'un maillage déformé, nous avons proposé de procéder à la construction des entités du modèle BRep correspondant en se basant sur le maillage de la peau (maillage surfacique) résultant d'un calcul élément (maillage déformé).

Cette méthode de reconstruction a buté sur plusieurs difficultés dont les plus importantes sont les suivantes :

- Extraction du maillage de peau à partir d'un maillage volumique ;

- Identification de la topologie du solide déformé à partir du maillage déformé, notamment dans le cas des grandes déformations (changement de géométrie et de topologie)

- Reconstructions des surfaces à partir d'un nuage de point irrégulier.

Deux approches ont été proposées et testées pour résoudre le problème de reconstruction de surface à partir d'une triangulation (maillage d'une face).

La première approche propose un algorithme, basé sur un support de points réguliers et la solution éléments finis (déplacements des noeuds), pour la reconstruction des surfaces. Cette approche est très limitée notamment en ce qui concerne la précision : les points d'interpolation de la surface sont situés sur le maillage qui ne présente pas toujours la géométrie réelle sauf en cas des faces planes. D'autre part, cette approche n'est pas applicable dans le cas de changement de topologie et dans le cas des surfaces avec un ou plusieurs contours intérieurs.

La deuxième approche est une amélioration de la première approche (approche $\mathrm{A}$ ) par exploitation des travaux Walton [Walton 96]. Cependant, les mêmes problèmes persistent avec les cas de la topologie inconnue et de faces avec contours intérieurs. Mais c'est une approche efficace et précise pour les modèles de topologie simple soumis à des sollicitations simples qui n'engendrent pas de changement de topologie.

\subsection{Retombées scientifiques des approches proposées.}

Nos travaux sur l'intégration des résultas des calculs éléments finis dans l'environnement de conception (CAO) ont fait l'objet d'un mastère soutenu et une thèse en cours, en collaboration avec le département de Génie Mécanique de l'Université du Québec à trois Rivières (Voir annexe II, Fiches des encadrements scientifiques : Mastère et Thèse de Borhène Louhichi).

Ces travaux ont abouti à plusieurs publications scientifiques nationales et internationales (voir Annexe I : Fiches des Publications Scientifiques) :

- B. Louhichi, A. BenAmara, V. François ; «Intégration CAO/Calcul par reconstruction des modèles $\mathrm{CAO}$ à partir des résultats de calcul» Revue Internationale d'Ingénierie Numérique, Volume $19-\mathrm{n}^{\circ}$ 1/2005. Éditions Hermès - Lavoisier 2005. 
- A. BenAmara, B. Louhichi, V. François; "CAD/Analysis integration: Building CAD models using FEA deformed mesh" Revue Computer Aided Design, soumis mai 2005.

- A. BenAmara, N. Aifaoui, B. Louhichi, V. François, D. Deneux; « Numerical integration and interoperability of CAD and analysis processes"; Revue Mécanique et Industrie ELSIVIER ; soumis avril 2005.

- V. François, B. Louhichi, A. BenAmara ; «Reconstruction d'un modèle CAO à partir d'un modèle éléments finis déformé », XVII Congrès Français de Mécanique, UTT, Troyes, France, 29 Août - 02 septembre 2005.

- B. Louhichi, N. Aifaoui, A. BenAmara, V. François, D. Deneux ; «Intégration numérique et interopérabilité des processus de CAO et de Calcul », XVII Congrès Français de Mécanique ; UTT -Troyes, France, 29 Août - 02 septembre 2005.

- B. Louhichi, A. BenAmara, V. François, L. Romdhane; «Rebuilding of CAD Model Starting from the Finite Elements Results» Le 20e Congrès Canadien de Mécanique Appliquée : CANCAM 2005 ; Université de McGill ; Canada, 30 mai au 2 juin 2005.

- B. Louhichi, V. François, A. BenAmara ; «Intégration de la méthode des éléments finis dans le processus de $\mathrm{CAO}$ : détermination de la géométrie d'un modèle déformé » $73^{\mathrm{e}}$ Congrès de l'ACFAS, Université du Québec à Chicoutimi, Canada, du 9 - 13 mai 2005.

- B. Louhichi, A. BenAmara, V. François ; «Reconstruction d'un modèle BREP déformé à partir des résultats d'une simulation éléments finis » Premier Congrès International Conception et Modélisation des Systèmes Mécaniques: CMSM2005; 23-25 Mars 2005; Hammamet - Tunisie.

- B. Louhichi, A. BenAmara, V. François ; « Simulation cinématique d'un assemblage dans les conditions de fonctionnement» $72^{e ́}$ Congrès de l'ACFAS, Université du Québec à Montréal, Canada, 10 - 14 mai 2004. 


\section{Plateforme de développement dédiée à Intégration CAO/Calcul.}

Ce travail rentre dans le cadre d'une collaboration avec le Professeur V. François de l'Université du Québec à Trois Rivières au canada et a fait l'objet du Mastère de Mr. Khaled Souaissa (Annexe II : Fiches d'encadrement Scientifique), co-dirigé avec Mr. Hedi BelHadjSalah, du Laboratoire de Génie Mécanique de l'Ecole Nationale d'Ingénieurs de Monastir.

L'objectif global de cette collaboration est la mise au point et le développement d'une plateforme commune dédiée à l'intégration des outils de $\mathrm{CAO}$ et de Calcul par éléments finis. Cela consiste à mettre en commun des bibliothèques et d'algorithmes de calcul en vue de les utiliser dans le cadre de nos travaux sur l'intégration $\mathrm{CAO} / \mathrm{Calcul}$.

La plateforme est constituée d'un ensemble de bibliothèques informatiques (bien structurées et documentées) capables, non seulement de se connecter sur un système de CAO et de générer un maillage optimisé et parfaitement intégré à la $\mathrm{CAO}$ (lien de dépendance entre le modèle $\mathrm{CAO}$ et structure de données maillage et conditions aux limites), mais aussi d'effectuer un calcul élément finis élastoplastique.

Notons que par rapport à l'utilisation des logiciels de calcul standards (COSMOS, ANSYS, ABAQUS), cette plateforme offre la possibilité d'un accès sans limites à l'ensemble des informations caractéristiques du processus de modélisation : CAO, maillage et calcul. La disponibilité des codes sources de l'ensemble des ressources développés pourra également constituer un avantage par rapport à l'utilisation des systèmes standards clé en main.

Dans un premier temps nous avons lancé, sur ce sujet, un mastère ayant pour objectif le développement d'un solveur élastoplastique tridimensionnel à l'aval du mailleur MAGIC développé à I'UQTR. Rappelons que ce mailleur permet, d'une part, de générer un maillage tridimensionnel optimisé, et d'autre part, un remaillage focalisé uniquement sur les parties modifiées du modèle CAO lors d'une modification topologique ou géométrique sur ce dernier. Ce qui répond aux exigences d'intégration $\mathrm{CAO} / \mathrm{Calcul}$, notamment en terme de gain de temps.

Le calcul élastoplastique envisagé dans le cadre de ce projet est basé sur les algorithmes de calculs élastoplastiques développés à notre Laboratoire LGM.

\subsection{Présentation de l’algorithme général de solveur élastoplastique}

L'algorithme utilisé dans ce travail [BelHadjSalah 03], constitue une nouvelle approche de résolution des problèmes élastoplastiques en calcul structures dans le cadre de l'hypothèse des petites perturbations avec écrouissage isotrope. Cet algorithme de calcul permet de donner la réponse élastoplastique d'une structure par la résolution d'un système plastique discret. On décompose le problème global en deux problèmes distincts (problème élastique et un problème résiduel). On résout le premier problème avec un premier incrément de charge purement élastique une seule fois. Les solutions élastiques en contrainte et en déplacement seront additionnées linéairement dans chaque incrément de charge.

\subsubsection{Algorithme du solveur Elastique}

La résolution d'un problème de calcul de structure élastique linéaire, est basée sur la résolution d'un système linéaire. Il est formé de la matrice de rigidité globale de la structure notée $[\mathrm{K}]$ et le vecteur charge globale noté $\{\mathrm{F}\}$. La solution de ce système permettra la connaissance de tous les degrés de liberté sur les nœuds présentés par le vecteur déplacement $\{U\}$.

Dans la résolution des problèmes élastiques, nous avons adopté l'approche déplacement. Les principales étapes de résolution au moyen de la méthode des éléments finis sont :

- Calcul élémentaire: Calculs des rigidités élémentaires $\left[\mathrm{K}^{\mathrm{e}}\right]$ et calculs des vecteurs forces élémentaires $\left\{\mathrm{F}^{\mathrm{e}}\right\}$ 
- Calcul global : Construction de la matrice de rigidité globale $[\mathrm{K}]$ et du vecteur force globale $\{\mathrm{F}\} . \quad[K]=\sum_{e}\left[k^{e}\right] \quad\{F\}=\sum_{e}\left\{F^{e}\right\}$

- Introduction des conditions aux limites (Méthode de pénalité)

- Résolution du système (méthode de Gauss)

La figure suivante (figure 46) montre les différentes étapes de la résolution d'un problème de calcul de structure élastique. A partir de la solution obtenue en déplacement, les solutions en contraintes et en déformations sont déduites systématiquement via la loi de comportement élastique et lois cinématiques qui relient les déformations aux déplacements.

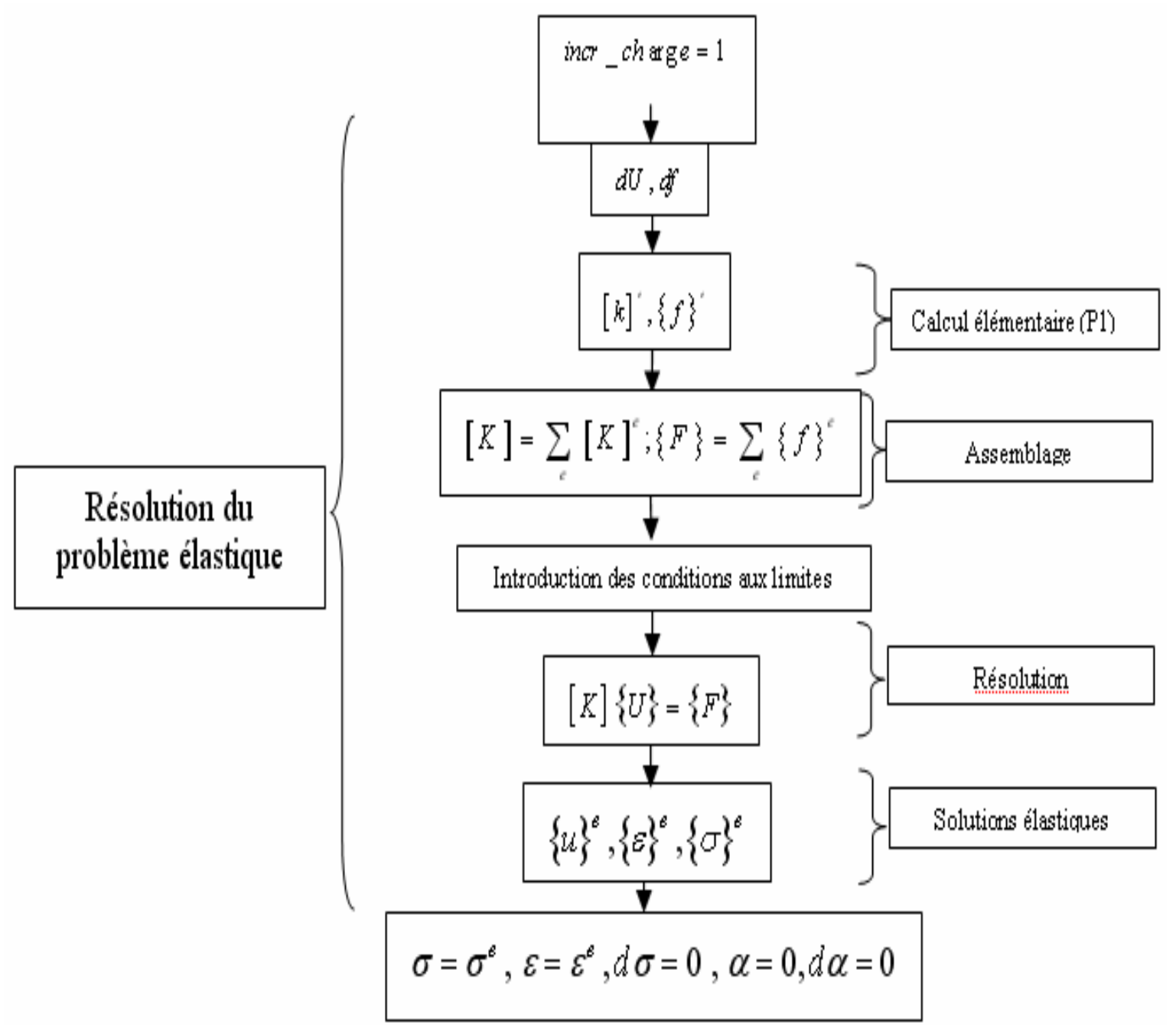

Figure 46 : Étapes de résolution d'un problème de calcul de structure élastique linéaire

\subsubsection{Algorithme du solveur élastoplastique.}

Pour un incrément de charge donné, on vérifie le critère de plastification sur chaque élément de maillage pour arriver à la fin de cet incrément lors de la résolution d'un système linéaire défini par la matrice $[\mathrm{M}]$ et le vecteur $[\mathrm{N}]$. La résolution de ce système permet de déterminer le vecteur des multiplicateurs plastiques de tous les éléments. Si un cœfficient est nul pour un élément, cela signifie que le chargement correspondant est purement élastique. S'il est non nul, l'élément se plastifie et à travers les équations d'écoulements plastiques on détermine les déformations plastiques. Enfin, la contrainte résiduelle des éléments plastifiés est calculée. 
Les deux solutions de deux problèmes s'associent pour former la solution globale d'un problème élastoplastique. Une mise à jour doit être effectuée sur tous les champs du problème, et qui seront pris en compte pour la résolution de l'incrément suivant.

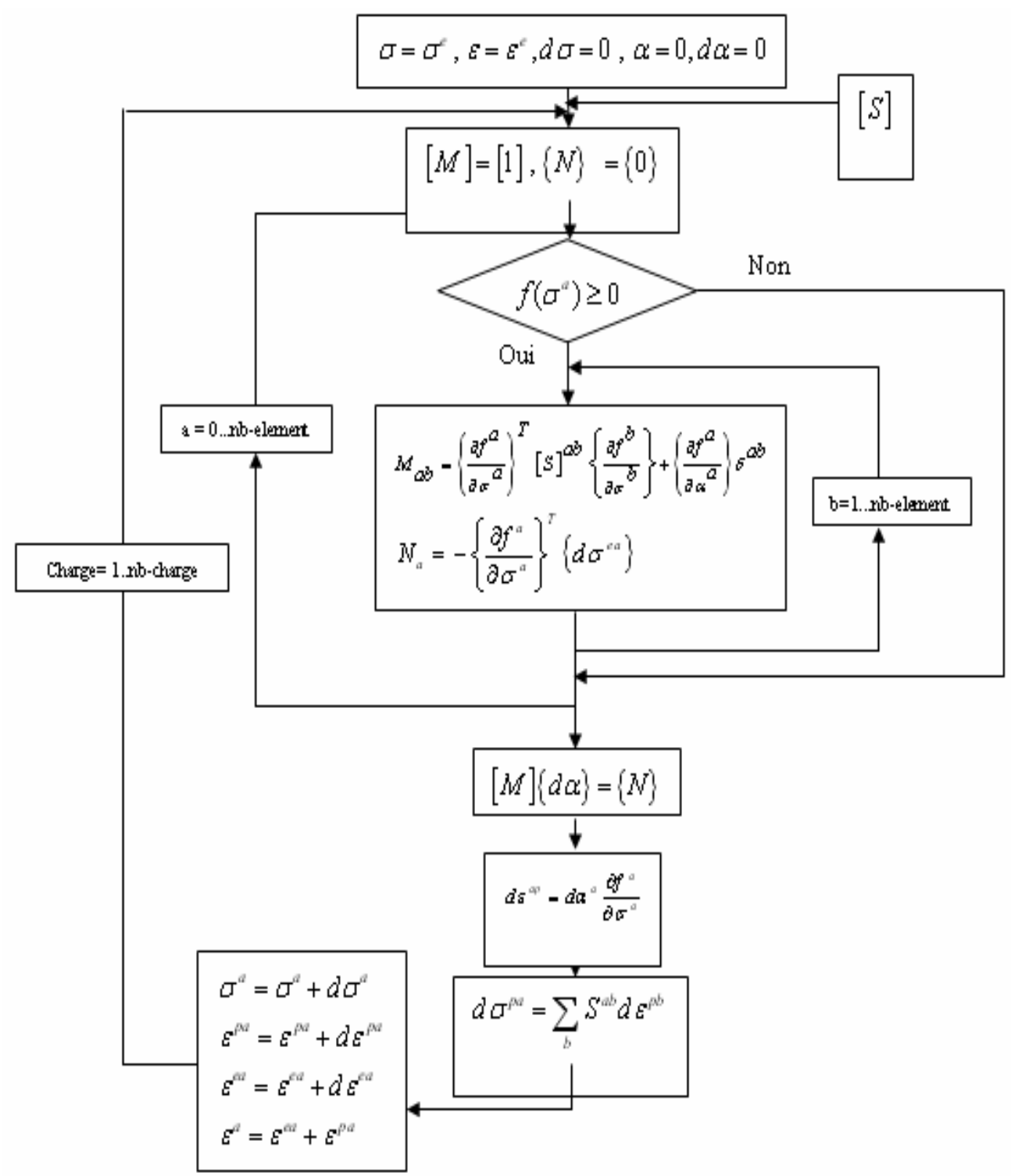

Figure 47 : Étapes de résolution d'un problème de calcul de structure élastoplastique

\subsection{Exemple de validation et confrontation des résultats avec des codes de calcul industriels}

Lors de la validation de ce travail, nous sommes partis des cas tests ayant des géométries et des conditions aux limites simples. C'est le cas des essais homogènes de traction ou bien de cisaillement appliqué sur un cube. Pour cet exemple, on commence par la validation de la partie élastique. Puis on passe à la validation de l'exemple plus raffiné. Une fois cette partie est validée, on abordera la validation du calcul élastoplastique.

La validation est également réalisée sur des exemples plus complexes (géométrie et conditions aux limites appliqués). C'est le cas de la bielle présentée ci-dessous.

\subsubsection{Essai de traction}

On considère un modèle géométrique représenté par un cube de dimension (100x100x100) discrétisé en 6 tétraèdres. Le matériau utilisé est un alliage d'acier caractérisé par un module de Young $\mathrm{E}=206.81 \mathrm{e} 03$ MPA et un cœfficient de Poisson $v=0.28$. 
La contrainte seuil $\sigma_{s}=230 \mathrm{MPa}$ et les conditions aux limites appliquées sont les suivantes :

- Blocage de trois faces selon la direction de leurs vecteurs normales.

- On applique une pression $\mathrm{P}=100 \mathrm{Mpa}$ sur la face de dessus.

La figure (48-a) présente la solution en déplacement selon la direction (z) de ce cas test calculé sur le solveur développé. La solution est visualisée sous MAGiC. À coté, figure (48-b) c'est la solution obtenue par le code de calcul ABAQUS 6.4.
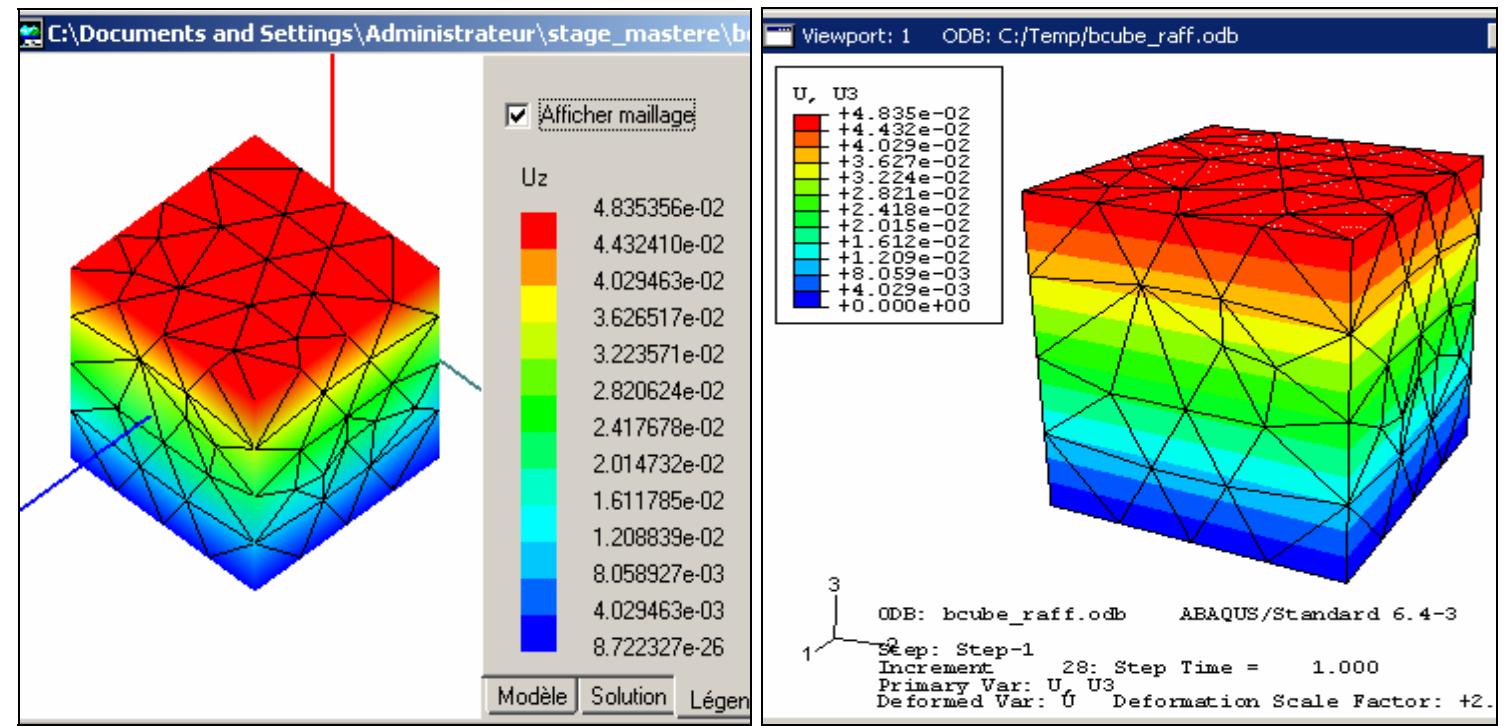

Figure 48 : Les isovaleurs des déplacements selon la direction z, calculées sur le solveur (a) développé et le logiciel ABAQUS (b).

Sur les figures suivantes $(49,50)$, on présente les courbes de réponses en contrainte en fonction de la déformation totale et de la déformation plastique d'un essai de traction homogène dont la face supérieure est soumise à un déplacement homogène de $10 \mathrm{~mm}$. De même les résultats obtenus sont confrontés aux résultats obtenus par ABAQUS.
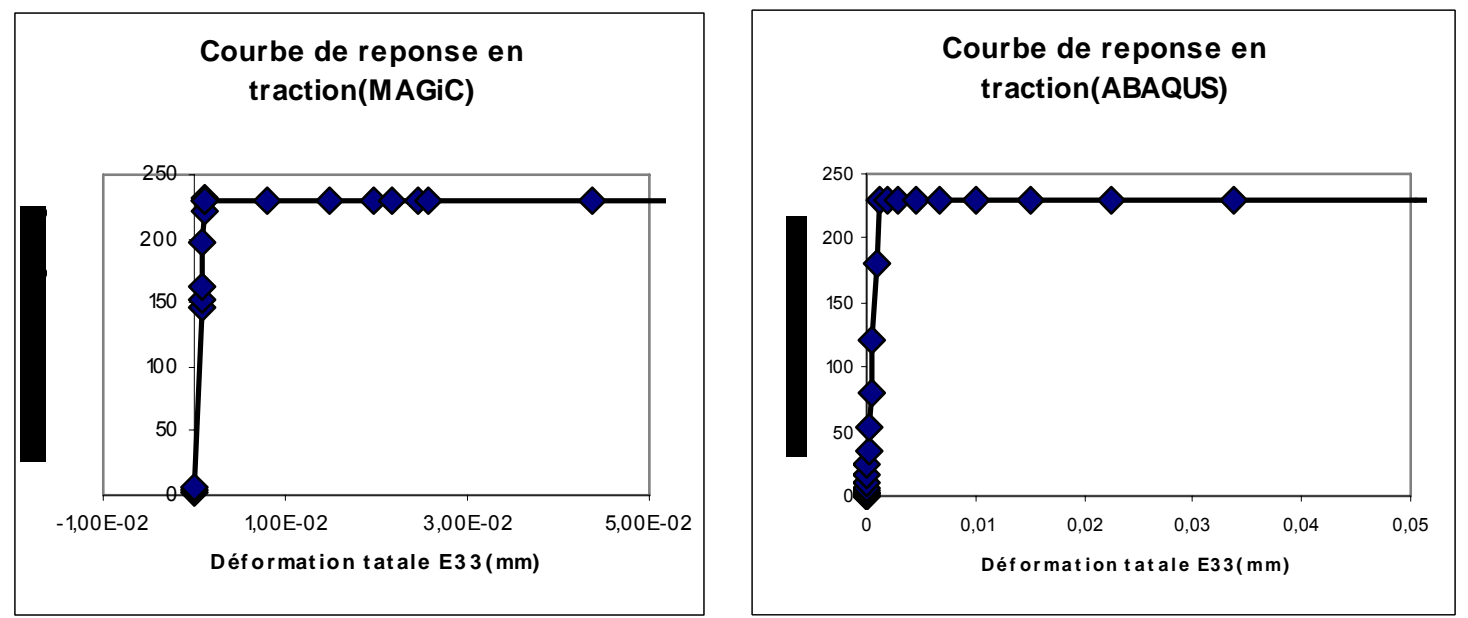

Figure 49 : Variation de la contrainte en fonction de la déformation totale.

\subsubsection{Exemple d'une bielle}

Dans la figure 50 ci-dessous, on présente un deuxième cas test (une bielle soumise à une pression de 100 Mpa et encastré au niveau du grand alésage). 

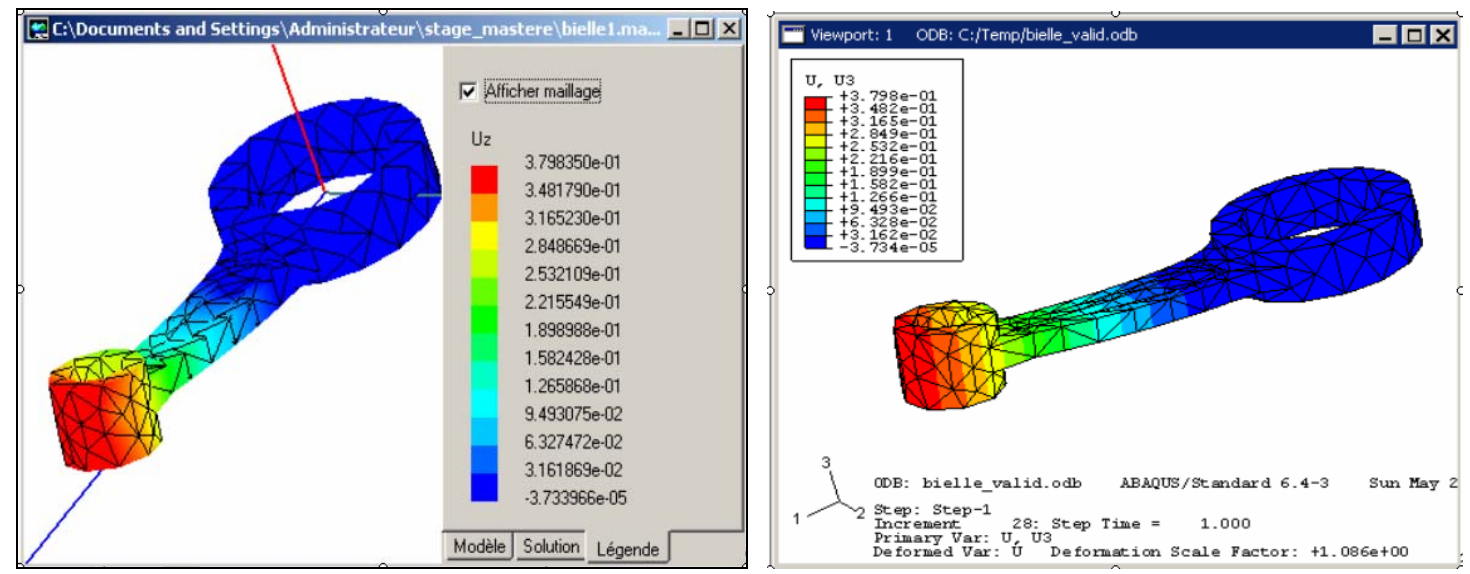

Figure 50 : Les isovaleurs des déplacements selon la direction z, calculée avec MAGiC (a) et ABAQUS (b).

\subsection{Avantages et limites du solveur élastoplastique proposé}

Ce travail constitue d'abord une plateforme de développement dédiée à l'intégration de la CAO et le Calcul par élément fini. Elle permettra de supporter nos futurs travaux dans ce domaine étant donné que nous disposons des codes source de toutes les bibliothèques de classes (maillage, résolution numérique, résultats de calcul : contraintes, déformations, etc.).

Elle permettra également de tester rapidement les propositions de recherche, notamment en ce qui concerne les méthodes de maillage, de calcul de structure élastoplastique, d'identification des lois de comportements, etc.

D'autre part elle permettra de développer d'autres travaux de recherche concernant l'intégration efficace du calcul dans le processus CFAO. En effet, nous envisageons d'intégrer de façon plus efficace la partie maillage et calcul avec le modèle CAO et nous projetons d'entreprendre ce calcul avec une optique de calcul parallèle distribué.

Cependant, en terme de temps de calcul CPU, notre algorithme de calcul n'est pas encore assez performant. L'amélioration de cet aspect nécessite l'optimisation du stockage de la matrice de rigidité. Il fallait la stoker en ligne de ciel et d'effectuer des renumérotation à fin d'optimiser la largeur de la bande, mais par soucis de validation du fonctionnement de la maquette, nous n'avons pas creusé ce point..

Cette étude, est limitée au cas des matériaux plastiquement parfaits. Il est donc nécessaire d'étendre la résolution pour d'autres matériaux et d'intégrer d'autres lois de comportement.

\subsection{Retombées scientifiques des approches proposées.}

Nos travaux sur le développement de cette plateforme d'intégration numérique ont fait l'objet du mastère soutenue de Mr. Khaled Souaissa, en collaboration avec le département de Génie Mécanique de l'Université du Québec à trois Rivières (Voir annexe II, Fiches des encadrements scientifiques : Mastère de Khaled Souaissa).

Ce travail est en cours de perfectionnement (mai - Septembre 2005) dans le cadre d'un stage postmastère qu'effectue Mr Khaled Souaissa au département de Génie Mécanique de l'Université du Québec à trois Rivières.

Un papier scientifique est en cours d'élaboration sur les résultats de ce travail. 


\section{PARTIE IV :}

Synthèses des Travaux de Recherche Emergeants 


\section{PARTIE IV}

\section{Synthèse des Travaux de Recherche Emergeants}

\section{Introduction}

Dans cette partie, nous présentons une synthèse de nos travaux de recherche en émergence qui constitue une ouverture sur d'autres champs de la recherche en génie mécanique par rapport à la thématique « intégration $\mathrm{CAO} / \mathrm{Calcul}$ » qui constitue l'axe principal de nos activités de recherche.

Ces travaux peuvent être classés sous la thématique suivante :

\section{Modélisation et Etude Expérimentale et Numérique des Procédés de Fabrication Mécanique.}

Dans le cadre de cette thématique, nous sommes impliqués dans des actions d'encadrement scientifique de sujets de Mastère et de Thèse à l'ENIM.

Les travaux soutenus dans le cadre de cette thématique sont les suivants :

- Mastère de Mr. Abdessalem Chamekh; «Simulation numérique du procédé d'emboutissage des tôles minces »; co-encadré avec Mr Hedi BelHadjSalah ; soutenu le 05/04/2003.

- Mastère de Mr. Imed Hajjaji ; "Etude expérimentale et numérique du procédé du tournage dur »; soutenu le 13/05/2005.

- Mastère de Mr. Fathi Bouabda; "Modèle de conception des moule d'injection plastique basé sur les entités technologiques »; soutenu 02/03/2002.

Les travaux en cours dans le cadre de cette thématique sont les suivants :

- Thèse de Doctorat de Mr. Ramzi Fredj ; «Caractérisation mécanique de l'usinage en finition »; Septembre 2003 - présent.

- Mastère de Mr. Mihed BenSaid ; "Corrélation entre l'usure de l'outil et l'effort de coupe en fraisage 3 axes », Juin 2005 - présent.

Dans ce qui suit nous présentons une synthèse des travaux soutenus dans cette thématique.

\section{Simulation numérique du procédé d'emboutissage des tôles minces}

Dans le cadre de ce mastère, nous nous sommes intéressés plus particulièrement à l'identification des sources des erreurs de modélisation qui peuvent engendrer des écarts remarquables entre la simulation numérique par calcul éléments finis et l'approche expérimentale.

En principe, les simulations numériques en calcul mécanique sont censées représenter de façon fidèle et précise le comportement réel du produit mécanique étudié (déformation, cinématique, etc.). En effet, si les modèles sur lesquels se basent les simulations numériques est bien élaboré, ces simulations doivent reproduire les résultats expérimentaux.

Dans ce travail, co-encadré avec Mr. Hedi BelHadjSalah, nous avons essayer d'étudier les résultats issus de la simulation numérique (calcul éléments finis) de l'emboutissage des tôles minces en procédant à une confrontation de ces résultats avec celles issues des essais expérimentales.

Nous avons pu constater que les résultas des simulations ne coïncident pas parfaitement avec les résultas expérimentaux et nous avons tenté d'identifier les causes principales de ces écarts entre simulations et expériences. 
Dans ce qui suit, nous présentons la démarche suivie dans le cadre de ce travail et nous soulignons ses principaux résultats.

\subsection{Modèle Expérimental : Hypothèses et résultats}

Nous considérons l'essai d'expansion équibiaxée par gonflement hydraulique (Jovignot) avec matrice circulaire. C'est un essai de mise en forme à froid réalisé sur une tôle mince. La tôle, fixée sur ses bords, se déforme sous l'action d'une pression exercée par un fluide (figure 51). L'un des avantages importants de cet essai est qu'il se déroule presque sans frottement. En effet, une matrice bloque l'éprouvette et empêche tout glissement de ses bords.

Au cours de cet essai, le déplacement au pôle de l'éprouvette est mesuré (capteur de déplacement) en fonction de la pression du fluide utilisé pour gonfler la tôle (capteur de pression). Nous pouvons aussi mesurer le profil de l'éprouvette déformée ainsi que la distribution de son épaisseur.

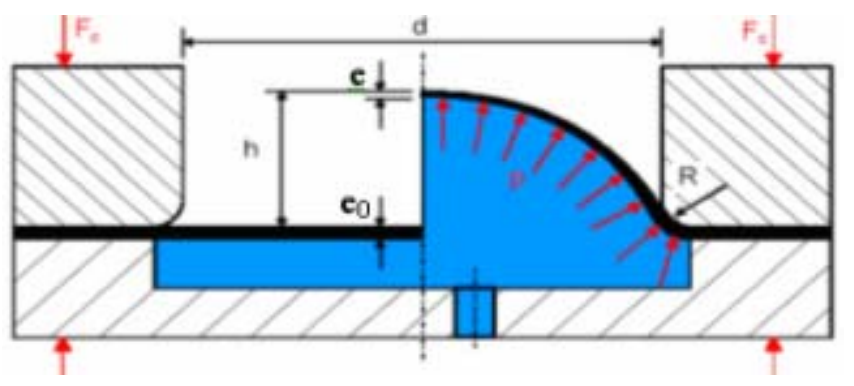

$$
\begin{aligned}
& \mathbf{d}=\mathbf{4 5 . 5} \mathbf{~ m m}: \text { Diamètre de la matrice } \\
& \mathbf{P}: \text { Pression du fluide } \\
& \mathbf{h}: \text { Hauteur au } \\
& \mathbf{F}_{\mathbf{c}}: \text { Force de serrage } \\
& \mathbf{R}=\mathbf{6 m m}: \text { Rayon de congé de la matrice } \\
& \mathbf{e}_{\mathbf{0}}=\mathbf{0 . 8} \mathbf{~ m m}: \text { Epaisseur initiale de l'éprouvette } \\
& \mathbf{e}: \text { Epaisseur au pôle de la tôle après } \\
& \text { déformation. }
\end{aligned}
$$

Figure 51 : Schéma de l'essai du gonflement hydraulique

\subsubsection{Hypothèses}

Le modèle d'étude considéré dans la modélisation de cet essai est basé sur les hypothèses suivantes :

- L'essai d'expansion par gonflement hydraulique est interprété comme un essai en contraintes planes dans le plan tangent à la tôle déformée. C'est un essai dans lequel prédominent les déformations en expansion.

- Le matériau de la tôle gonflée est l'acier INOX X6 Cr Ni 18-09.

- La courbe d'écrouissage de cet acier obtenue à partir de l'essai de traction simple est présentée à la figure3 ci-dessous :

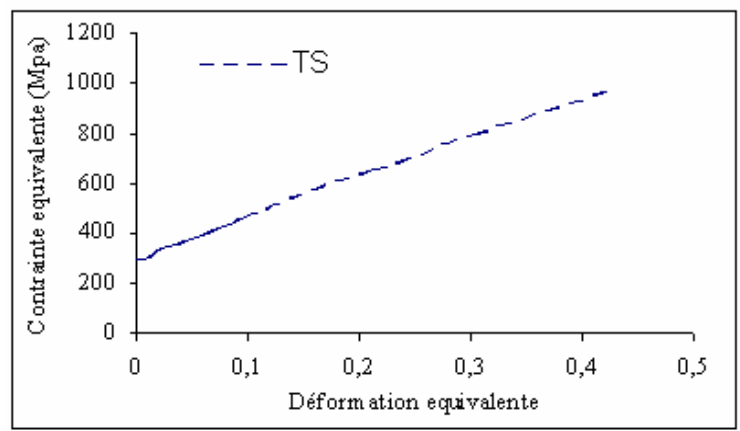

Figure 52 : Courbe d'écrouissage obtenue à partir d'un essai de traction simple

- Les coefficients de Lankford, mesurés pour les essais hors axes avec des angles hors axes $\psi=0^{\circ}, 45^{\circ}$ et $90^{\circ}$ sont donnés au tableau ci-dessous :

\begin{tabular}{|l|l|l|l|l|}
\hline \multirow{2}{*}{$R_{y}=\frac{d \varepsilon_{22}}{d \varepsilon_{33}}$} & $\mathrm{R} 0$ & $\mathrm{R} 45$ & $\mathrm{R} 90$ & \\
\cline { 2 - 6 } & 0.93 & 1.07 & 0.87 & 0.94 \\
\hline
\end{tabular}




\subsubsection{Résultat expérimental.}

La courbe ci-dessous présente la variation de pression interne du fluide en fonction de la hauteur au pôle de l'éprouvette.

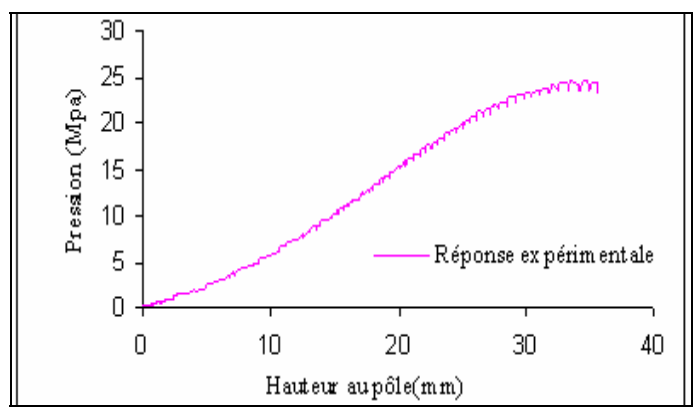

Figure 53 : Variation de la pression en fonction de la hauteur au pôle

\subsection{Modèle Numérique : Hypothèses et résultats}

Pour simuler l'essai de gonflement hydraulique en utilisant la méthode des éléments finis moyennant le logiciel ABAQUS, il est d'abord nécessaire de construire le modèle mécanique correspondant.

\subsubsection{Hypothèses}

Le modèle mécanique considéré pour la simulation numérique de cet essai est basé sur les hypothèses suivantes :

\subsubsection{Hypothèses géométriques}

La tôle est modélisée par une plaque mince circulaire de diamètre (D) et d'épaisseur $\left(\mathrm{e}_{0}\right)$. Cette plaque a été ensuite maillée en deux domaines volumiques : la partie centrale de la plaque à base d'un élément tétraédrique à 6 nœuds et la partie restante en utilisant l'élément cubique à 8 nœuds.

\subsubsection{Hypothèses de conditions aux limites}

La modélisation des conditions aux limites concerne, dans notre cas, la pression et l'effort de la matrice. La pression de gonflement appliquée sur la face inférieure de la plaque est supposée uniforme suivant un profil linéaire. Concernant l'effort de la matrice, deux cas de figure ont été simulés. En effet, nous avons modélisé cet effort, dans un premier temps, par un simple encastrement du périmètre de la tôle, et dans un second temps, en prenant en compte l'aspect contact entre la matrice et la tôle.

Concernant le premier cas de figure (figure 54), l'encastrement a été appliqué, d'abord, sur une circonférence de diamètre $d$, ensuite sur une circonférence de diamètre $d+R$, étant donné la géométrie du serre-flan (rayon de courbure R).

La prise en compte du frottement entre la tôle et la matrice au niveau du rayon du congé $\mathrm{R}$ (voir figure 51), a été modélise, d'abord, par un contact glissant, ensuite par un contact collant (figure 54).

Trois cas de conditions aux limites ont été considérés dans cette études :

- Avec encastrement sur le flanc à $\mathbf{d}=\mathbf{4 5 . 5} \mathbf{~ m m}(\mathrm{d}=$ Diamètre de la matrice $)$,

- Avec encastrement sur le flanc à $\mathbf{d}+\mathbf{R}=\mathbf{5 1 . 5} \mathbf{~ m m}(\mathrm{d}=$ Diamètre de la matrice, Rayon de congé de la matrice),

- Avec prise en compte du frottement. 


\subsubsection{Hypothèses de comportement du matériau}

Le matériau considéré est l'acier inox X6 Cr Ni 18-09 caractérisé par les coefficients de Lankford donnés par le tableau ci-dessus. Nous avons utilisé le modèle de comportement élastoplastique formulé en grande déformation implémenté dans le code Abaqus, qui utilise les critères de Hill et de Von Mises.
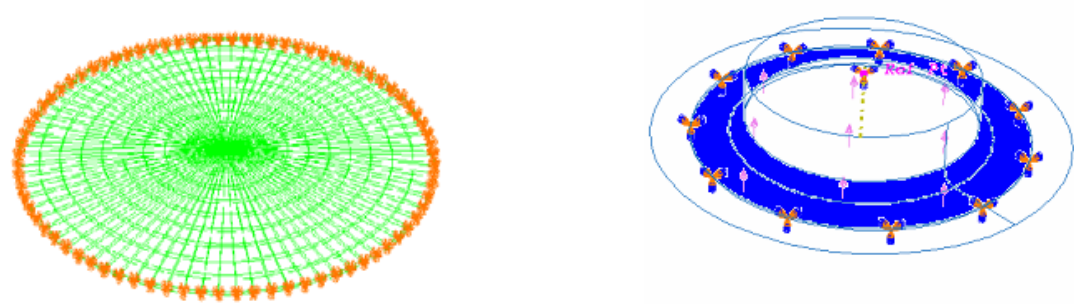

Figure 54 : Modèle numérique sous Abaqus.

\subsubsection{Résultats et interprétations}

Le résultat du calcul éléments finis sur Abaqus permet de représenter les isovaleurs des déplacements, des déformations équivalentes et des contraintes équivalentes de Von Mises.

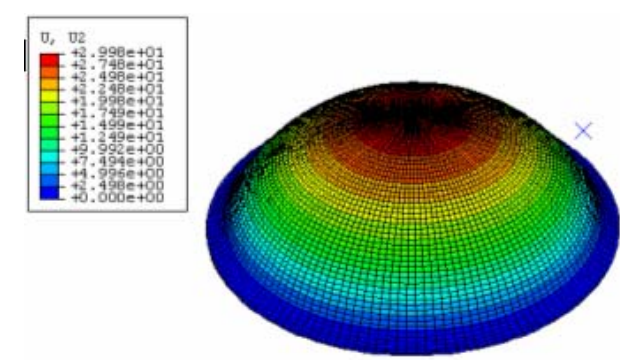

Figure : Isovaleurs de Déplacements

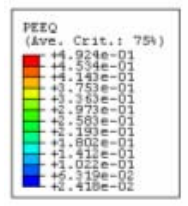

Figure : Isovaleurs de

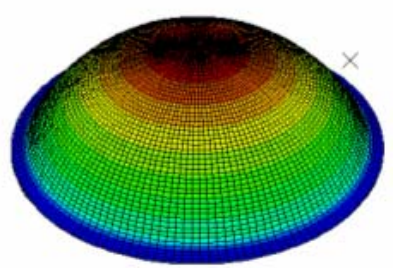

équivalente de Von Mises
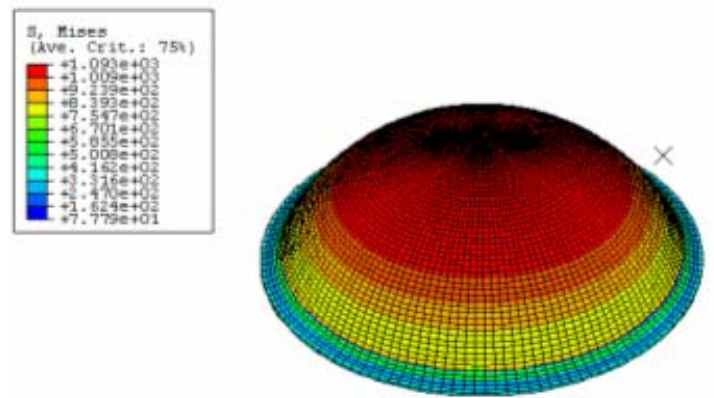

Figure 55 : Isovaleurs de $\sigma$ équivalente de Von Mises

La comparaison entre la réponse expérimentale pression-déplacement et les réponses prédites numériquement pour ces trois modèles considérés est présentée dans la figure ci-dessous.

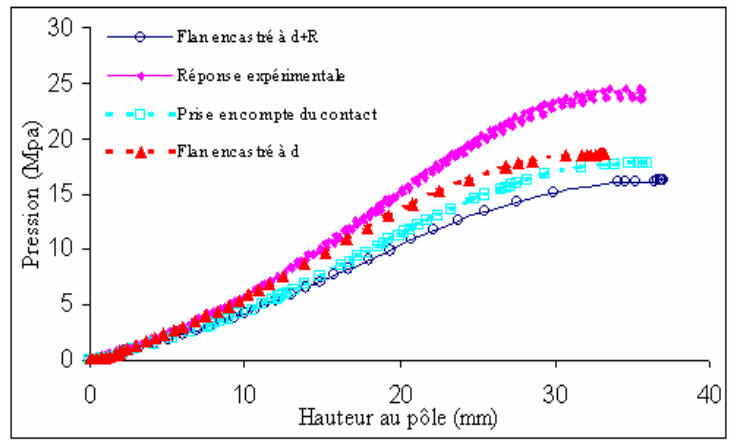

Figure 56 : Variation de la hauteur au pôle en fonction de la pression (Critère de Von Mises) 
D'après la figure 56, on voit clairement que pour les trois cas de conditions aux limites précités, l'écart entre les deux réponses expérimentale et numérique croit avec la pression.

Pour exclure toute erreur dans nos simulations nous avons menés plusieurs calculs en tenant compte de l'effet de plusieurs paramètres :

En effet, d'après [GAIECH 01] la raison de l'écart trouvé entre la réponse expérimentale et la réponse numérique est l'effet du nombre de couches d'éléments du maillage en épaisseur. Nous avons effectué des calculs avec une, deux et trois couches, et nous avons trouvé que les courbes pression en fonction de la hauteur au pôle sont quasi-identiques [CHABENAT 03]. Ce qui permet de rejeter cette possibilité.

Nous avons étudié aussi l'influence des conditions aux limites en prenant trois modèles géométriques de l'essai. Nous avons constaté l'existence de cet écart dans les trois cas. D'autre part nous avons tenu compte de la faible anisotropie de l'acier Inox et nous avons remarqué qu'en appliquant les critères de Hill et celui de Von Mises nous trouvons presque la même réponse. Il faut noter, aussi, que le modèle de comportement élastoplastique anisotrope couplé à l'endommagement isotrope formulé en grandes déformations dans [BOULILA 00] n'a pas permis de réduire significativement l'écart entre les réponses expérimentale et numérique.

Nous pensons que cet écart entre les réponses numérique et expérimentale vient de l'identification de la courbe d'écrouissage à travers l'essai de traction simple, pour cela nous proposons d'identifier cette courbe à partir de l'essai d'expansion équibiaxiale. Pour cela nous avons appliqué le modèle en calotte sphérique pour exprimer la contrainte et la déformation équivalente, au pôle, au sens de Von Mises en fonction de la pression et de la hauteur au pôle.

La démarche suivie est présentée dans les paragraphes suivants :

Contrainte équivalente : Le matériau testé (acier inoxydable) obéit au critère de Von Mises. Pour ce cas, la contrainte équivalente se présente sous la forme :

$$
\sigma=\sqrt{\frac{1}{2}\left\{\left(\sigma_{1}-\sigma_{2}\right)^{2}+\left(\sigma_{1}-\sigma_{3}\right)^{2}+\left(\sigma_{2}-\sigma_{3}\right)^{2}\right\}}
$$

Où $\sigma_{1}, \sigma_{2}$ et $\sigma_{3}$ représentent les contraintes principales.

Pour ce qui est du modèle d'une déformée en calotte sphérique, la contrainte $\sigma_{1}$ correspond à la contrainte méridienne, $\sigma_{2}$ correspond à la contrainte circonférentielle et $\sigma_{3}$ représente la contrainte normale qui est nulle dans notre cas.

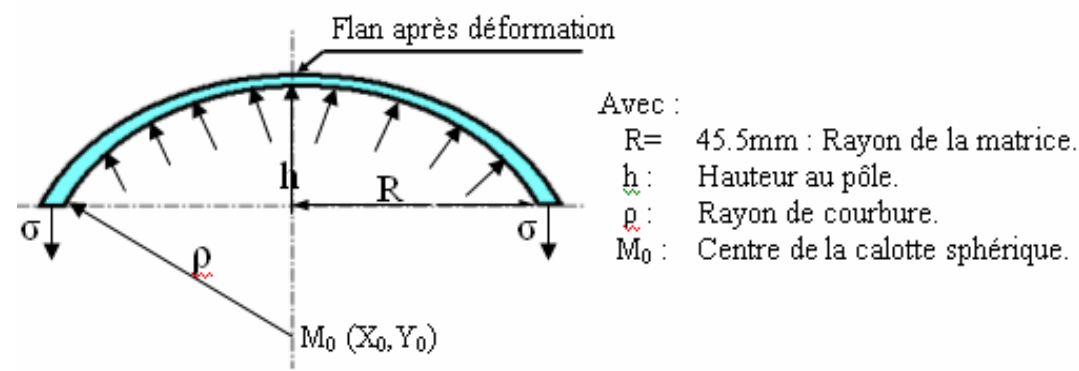

Figure 57 : Equilibre du tronçon de la calotte sphérique

D'après l'équilibre de la calotte sphérique, on trouve $\sigma=\frac{P . \rho}{2 e}$, avec $\rho=\frac{R^{2}+h^{2}}{2 h}$ et $\mathrm{e}=\mathrm{e}_{0}\left(1+\frac{h^{2}}{R^{2}}\right)^{-2} ;$

Ce qui donne la contrainte équivalente : $\quad \sigma=\frac{P\left(R^{2}+h^{2}\right)^{3}}{4 e_{0} h R^{4}}$ 
Déformation équivalente : Comme pour la contrainte équivalente, la déformation plastique équivalente de Von Mises, est donnée par :

$$
\varepsilon=\sqrt{\frac{2}{3}\left(\varepsilon_{1}^{2}+\varepsilon_{2}^{2}+\varepsilon_{3}^{2}\right)} \quad \text { où } \varepsilon_{1}, \varepsilon_{2} \text { et } \varepsilon_{3} \text { représentent, respectivement, les déformations }
$$

principales méridienne, circonférentielle et normale.

D'après le modèle en calotte sphérique on a

$$
\varepsilon_{1}=\varepsilon_{2} \text { on a d'autre part } \varepsilon_{3}=-2 \varepsilon_{1}=\log \left(\mathrm{e} / \mathrm{e}_{0}\right)=\log \left(1+\frac{h^{2}}{R^{2}}\right)
$$

Ce qui donne la déformation équivalente :

$$
\varepsilon=2 \log \left(1+\frac{h^{2}}{R^{2}}\right)
$$

Nous avons, par suite, pu reconstituer la courbe d'écrouissage a partir de la courbe pression en fonction de la hauteur au pôle (figure 58) :
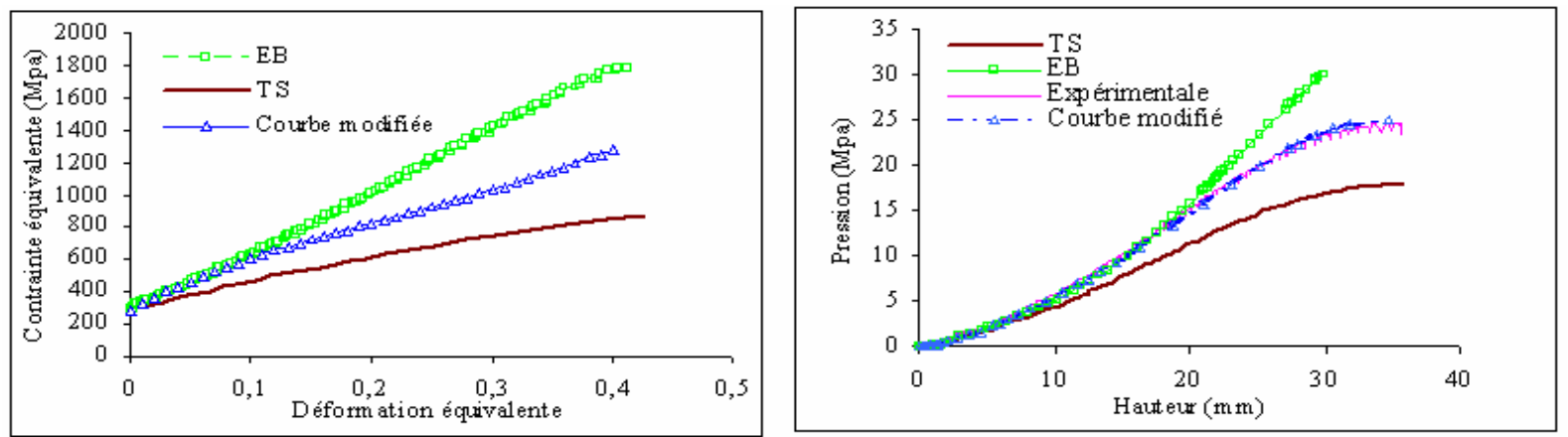

Figure 58 : Courbes d'écrouissage issues de la traction simple et de l'essai équibiaxiale et comparaison entre les réponses pression-déplacement.

D'après la figure ci-dessus, on constate que, la réponse numérique pression-déplacement, surestime la réponse expérimentale, dans le cas d'une courbe d'écrouissage issue d'un essai d'expansion équibiaxiale, et le sous-estime dans le cas d'une courbe d'écrouissage relevé d'un essai de traction simple. Pour cela nous avons ajusté la courbe d'écrouissage en vue de caler les deux réponses.

\subsection{Retombées scientifiques des approches proposées.}

Ce travail a fait l'objet d'un Mastère de Génie Mécanique au sein du Laboratoire de Génie Mécanique de l'Ecole Nationale d'Ingénieurs de Monastir sous l'encadrement scientifique de Mr. Hedi BelHadjSalah et moi-même (Voir annexe II, Fiches d'Encadrement Scientifique : Mastère de Mr. Abdesslem Chamekh).

Ce travail a abouti à la publication d'un article de revue et une communication nationale. (voir Annexe I : Fiches Publications Scientifique) :

\section{Article de revue :}

- A. Chamekh, H. BelHadjSalah, M. A. Gahbiche, A. BenAmara, A. Dogui, "Identification of the hardening curve using a finite element simulation of the bulge test" Steel GRIPS Journal of steel and related materials; Septembre 2004 741-746 (2004)

\section{Communication Nationale :}

- A. Chamekh, M. A. Gahbiche, H. Bel Hadj Salah, A. BenAmara \& A. Dogui, "Etude expérimentale et numérique de l'essai de gonflement hydraulique", Actes des JS2003, T2, pp 222-227, 21-22 mai (2003), Borj Amri, Tunisie. 


\section{Etude expérimentale et numérique du tournage dur}

Ce travail porte sur l'étude expérimentale et numérique du tournage dur. Ce travail a débuté par une analyse bibliographique sur ce procédé qui nous a permis de cerner les différents paramètres intervenant dans la caractérisation de la qualité d'usinage pour ce procédé.

Une étude expérimentale est ensuite menée sur l'acier X155CrMoV12 durci par traitement thermique à 57 HRC. L'outil de coupe employé est en CBN de nuance CB7020 avec une géométrie de coupe chanfreiné. L'objectif de cette étude est l'analyse de l'évolution de la rugosité de la surface, de l'effort de coupe et de l'usure (formes et mécanismes) en fonction des paramètres de coupe (vitesse de coupe, vitesse d'avance, profondeur de passe).

Une étude numérique est développée à l'aide du code de calcul Abaqus. L'objectif de cette étude est d'analyser le comportement cinématique de l'outil de coupe sous l'effet des efforts de coupe ainsi que les paramètres influents.

\subsection{Dispositif Expérimental}

Ci-dessous, nous présentons le dispositif expérimental qui sert à l'étude des évolutions des efforts, de la rugosité et de l'usure ainsi que de la procédure d'acquisition des données. Nous présentons également l'outillage utilisé ainsi que la matière usinée.

\begin{tabular}{|c|c|c|c|c|}
\hline \multirow{3}{*}{$\begin{array}{l}\text { Matériels de mesure : } \\
\qquad \begin{aligned} & \text { Banc de mesure Kistler } \\
& \text { - Rugosimètre } \\
& \bullet \text { Loupe optique }\end{aligned}\end{array}$} & \multicolumn{4}{|c|}{$\begin{array}{l}\text { Matière : } \\
\text { • X155 Cr MoV12 durcis } \\
\text { - Thermiquement à } 57 \text { HRC }\end{array}$} \\
\hline & $\mathrm{C}$ & $\mathrm{Cr}$ & Mo & $\mathrm{V}$ \\
\hline & $1,4-1,6$ & $11-12$ & $0,7-1,2$ & Max 1,1 \\
\hline $\begin{array}{l}\text { Outil de coupe : } \\
\qquad \begin{array}{l}\text { - Plaquette : CNGA } 120408 \\
\text { S 01020, en CBN (Nitrure de Bore } \\
\text { Cubique) nuance CB7020 }\end{array}\end{array}$ & \multicolumn{4}{|c|}{$\begin{array}{l}\text { Porte plaquette : } \\
\quad \text { - CCLNR 25252M 12-IC }\end{array}$} \\
\hline
\end{tabular}

\subsection{Procédure des essais}

Notre étude expérimentale consiste à réaliser des opérations de chariotage sur des éprouvettes cylindriques (de diamètre $D=45 \mathrm{~mm}$ ) en acier traité, afin d'étudier la variation des efforts de coupe, de l'usure de l'outil, et de la rugosité de la surface en fonction des paramètres de coupe : vitesse de coupe, avance et profondeur de passe.

Pour réussir ces essais, nous avons adopté une méthode d'analyse statistique des résultats recueillis. Un plan d'expérience est dressé pour bien mener cette étude expérimentale. Les variables de ce plan sont :

- La vitesse de coupe Vc ;

- L'avance f ;

- La profondeur de passe ap .

Les paramètres mesurés sont :

- Les efforts de coupe : Effort tangentiel Fc, de pénétration Fp et d'avance Fa ;

- La rugosité arithmétique moyenne ;

- L'usure de l'outil ; 


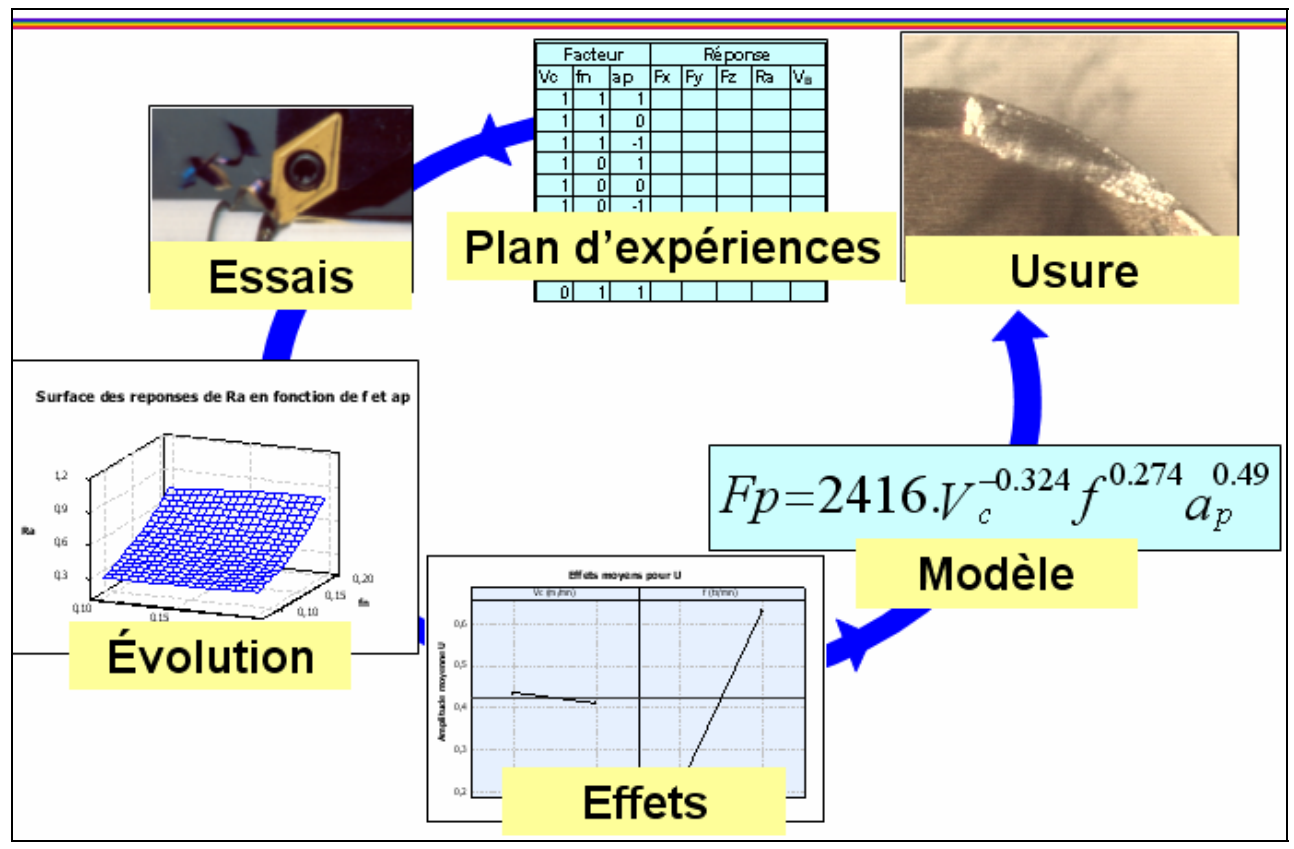

Figure 59 : Procédure Expérimentale.

\subsection{Etude de l'évolution de la rugosité de la surface usinée}

L'état de la surface en tournage dur est l'un des critères d'évaluation des performances de ce procédé d'usinage. La formule théorique donnant la rugosité de la surface en fonction des paramètres de coupe montre que la rugosité dépend essentiellement de l'avance $f$ (théoriquement on a $R t=f^{2} / 8 R_{\varepsilon}$ ).

Les surfaces d'évolution de la rugosité en fonction des paramètres de coupe (Vc, $f$ et ap) sont présentées dans les graphiques suivants.

\section{Surface des reponses de Ra en fonction de fn et Vc}
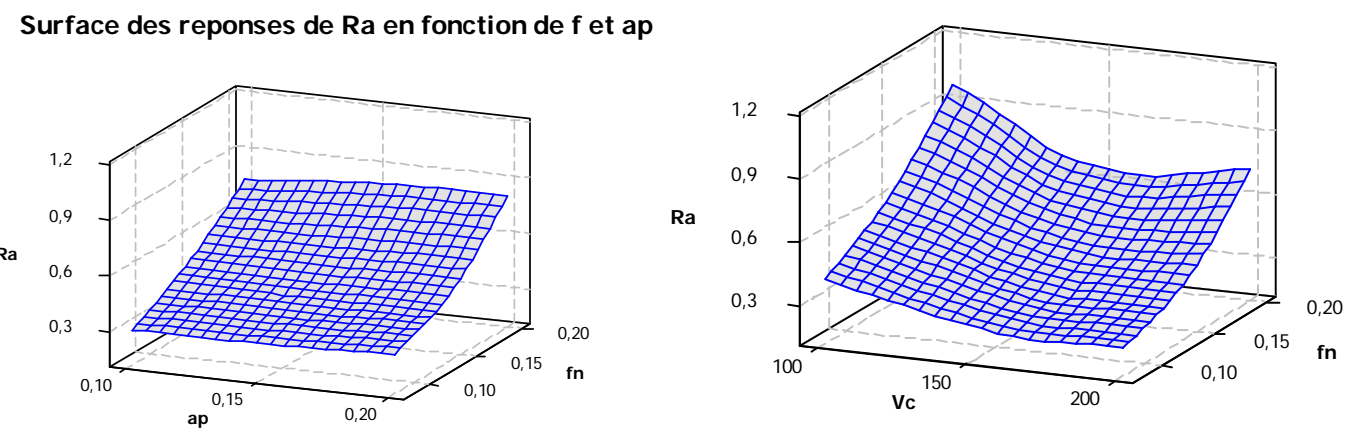

Figure 60 : Evolution de la rugosité de la surface en fonction de fet ap et de fn et Vc

Ces graphiques montrent que l'avance a un effet significatif sur la rugosité. En effet Ra passe de $0.37 \mu \mathrm{m}$ à $0.93 \mu \mathrm{m}$ lorsque l'avance varie de $0.08 \mathrm{~mm} / \mathrm{tr}$ à $0.2 \mathrm{~mm} / \mathrm{tr}$, (pour une vitesse de coupe de $100 \mathrm{~m} / \mathrm{mn})$.

Aussi, on constate qu'une augmentation de la vitesse de coupe améliore la rugosité de la surface. En effet, la rugosité $R a$ passe de $0.37 \mu \mathrm{m}$ pour $V C=100 \mathrm{~m} / \mathrm{mn}$ à $0.2 \mu \mathrm{m}$ pour $V_{C}=200 \mathrm{~m} / \mathrm{mn}$ (ceci pour une avance de $0.1 \mathrm{~mm} / \mathrm{tr}$ ).

On peut aussi conclure que l'effet de l'avance est plus important par rapport à celui de la vitesse de coupe, en effet les variations de la surface des réponses de Ra sont plus accentuées avec la variation de $f$ qu'avec celle de Vc. 
Ce graphique montre aussi que la surface des réponses de la rugosité est pratiquement invariante avec la variation de la profondeur de passe ap. (La rugosité Ra varie légèrement avec la variation de ap). Ces constatations sont confirmées par la bibliographie [Özel 03] [Feng 03].

\subsubsection{Modèle de prédiction de la rugosité :}

Dans la suite, on présente la méthodologie de la modélisation de la variation de Ra en fonction des paramètres $(V c, f$, et $a p)$. Généralement, les modèles issus des plans d'expérience s'écrivent sous forme polynomiale [Montogomery 97]. Le modèle décrivant la variation de Ra s'écrit sous la forme suivante :

$$
R a=k_{0}+k_{1} \cdot V c+k_{2} \cdot f+k_{3} \cdot a p+k_{11} \cdot V c^{2}+k_{22} \cdot f^{2}+k_{33} \cdot a p^{2}+k_{12} \cdot V c \cdot f+k_{13} \cdot V c \cdot a p+k_{23} \cdot f \cdot a p
$$

Ce modèle peut être simplifié par l'élimination des termes dont l'effet est statistiquement faible. Une première analyse des effets permet de déterminer la probabilité pour qu'un facteur soit statistiquement influent.

D'après cette analyse on a pu conclure que les effets de $V c, V c^{2}$ et $f^{2}$ sont significatifs. Les autres termes du modèle ne présentent pas d'effets considérables.

Ce modèle permet de prédire les valeurs de $\mathrm{Ra}$ en fonction de paramètres de coupe avec un coefficient de détermination de $82.2 \%$.

Donc le modèle de prédiction de $R a$ s'écrit de la façon suivante :

$$
R a=1,62788-0,01647 . V c+0,00005 . V c^{2}+20.132 . f^{2}
$$

Le modèle exponentiel de $R a$ en fonction de $f$ et $V c$ peut s'écrire de la façon suivante

$$
R a=c_{0} \cdot V^{c 1} \cdot f^{c 2}
$$

L'application de la fonction logarithmique $(L n)$ permet de transformer l'équation non linéaire en une équation linéaire

$$
\operatorname{Ln}(\mathrm{Ra})=\ln \left(\mathrm{c}_{0}\right)+\mathrm{c}_{1} \cdot \ln (\mathrm{Vc})+\mathrm{c}_{2} \cdot \ln (\mathrm{f})
$$

\subsubsection{Validation du modèle proposé:}

La régression linéaire de $\ln (R a)$ permet la détermination des constantes. Tout calcul fait, on trouve : $\mathrm{Ra}=100.756 . \mathrm{Vc}^{-0.659} \cdot \mathrm{f}^{1.027}$

La figure 61 représente la comparaison entre la rugosité mesurée expérimentalement et celle calculée avec les deux modèles polynomial et exponentiel.

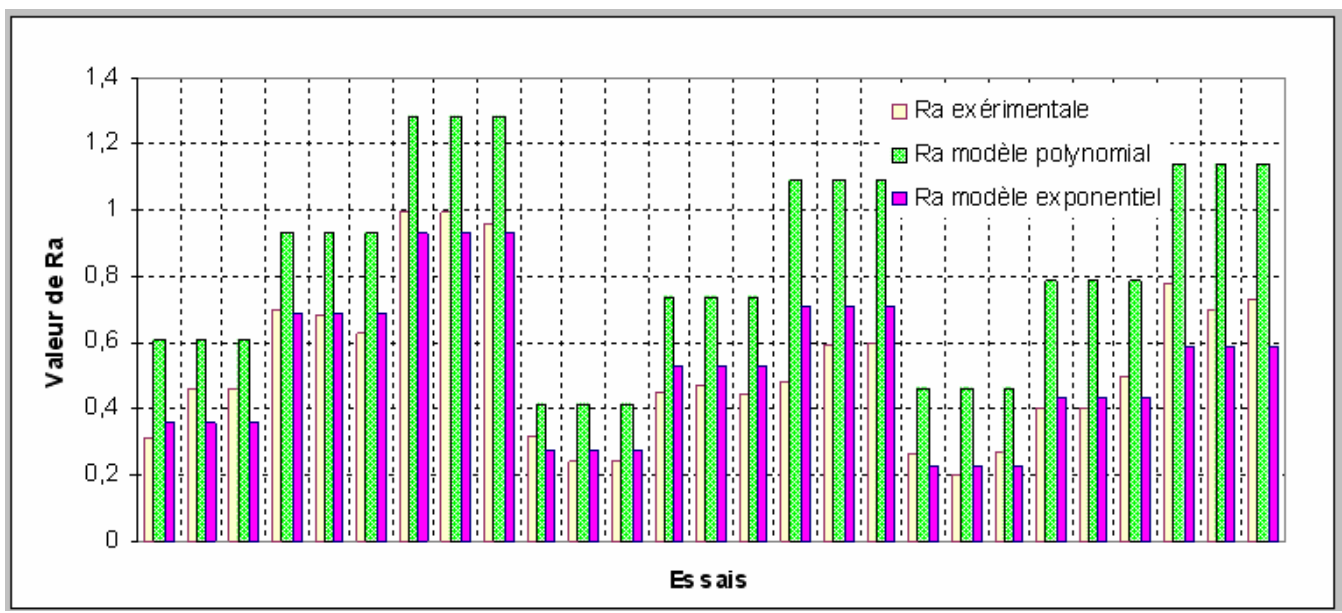

Figure 61 : Comparaison des valeurs expérimentales de Ra et celles prédites 
L'analyse de la figure 62 ci-dessus montre que le modèle exponentiel permet de prédire les valeurs expérimentales de $\mathrm{Ra}$ avec un écart type de l'erreur (différence de la valeur prédite et la valeur expérimentale) de $9.12 \%$, alors que le modèle polynomial présente un écart type de l'erreur de $11.96 \%$. Par conséquent, le modèle exponentiel est le plus fiable pour la détermination de Ra.

\subsection{Etude de l'effort de coupe et de l'usure}

L'étude des chargements de l'outil de coupe et de son usure est une étape primordiale pour maîtriser le procédé du tournage dur. Les efforts de coupe sont des indicateurs de la qualité de l'usinage et de l'état de l'usure de l'outil.

\subsubsection{Etude de l'effort de coupe}

Ci-dessous une synthèse de l'étude de l'évolution des composantes de l'effort de coupe ( $\mathrm{Fc}, \mathrm{Fp}, \mathrm{Fa})$ en fonction des paramètres d'usinage (vitesse de coupe $V c$, avance $f$ et profondeur de passe $a_{p}$ ). L'objectif est d'optimiser les conditions de coupe et le domaine d'application de ce procédé.

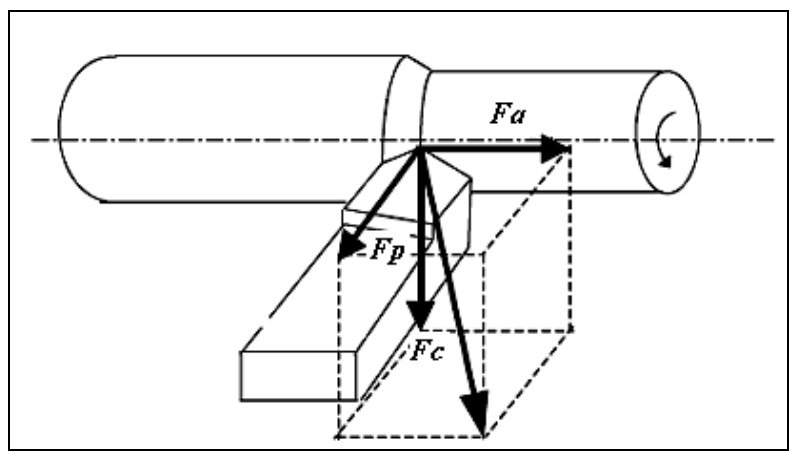

Figure 62 : Schématisation des efforts de coupe

\subsubsection{Evolution des efforts de coupe en fonction de la vitesse de coupe}

Dans cette partie nous présentons l'influence des la vitesse de coupe $V c$ sur les trois composantes de l'effort de coupe ( $F p, F C$ et $F a)$.

Les valeurs des trois composantes de l'effort de coupe décroissent avec des pentes différentes lorsque Vc augmente (figure 63). C'est l'effet grande vitesse [Poulachon 99]. L'augmentation de Vc engendre un travail mécanique supplémentaire qui adoucit le métal dur et par la suite sa résistance à la coupe diminue, d'où les efforts de coupe sont plus faibles.

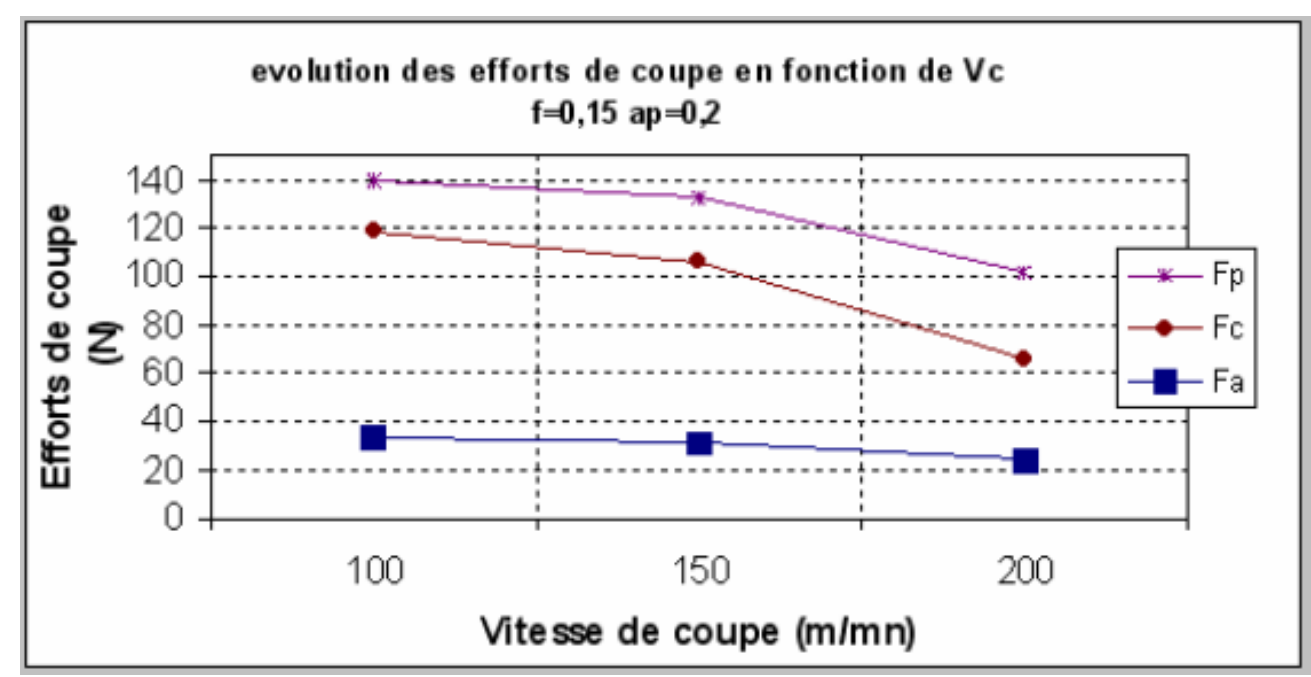

Figure 63 : Evolution des efforts de coupe en fonction de Vc 

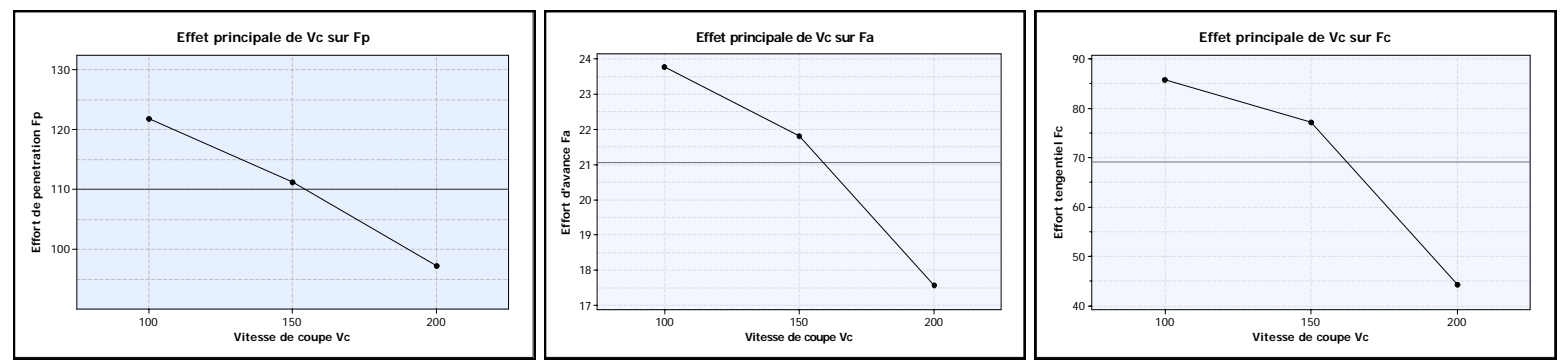

Figure 64 : Graphique des effets de la vitesse de coupe sur les efforts

Les graphiques précédents représentent les courbes des effets de la vitesse de coupe sur les trois composantes de l'effort de coupe. On peut remarquer que l'effet de la vitesse de coupe Vc est plus important sur la composante tangentielle et radiale.

\subsubsection{Evolution des efforts de coupe en fonction de l'avance}

Les efforts de coupe suivent une allure croissante en fonction de l'augmentation de l'avance $f$, mais cette évolution n'a pas la même intensité pour les trois composantes de l'effort de coupe. L'effort radial $\mathrm{Fp}$ est le plus sensible à la variation de la vitesse d'avance.

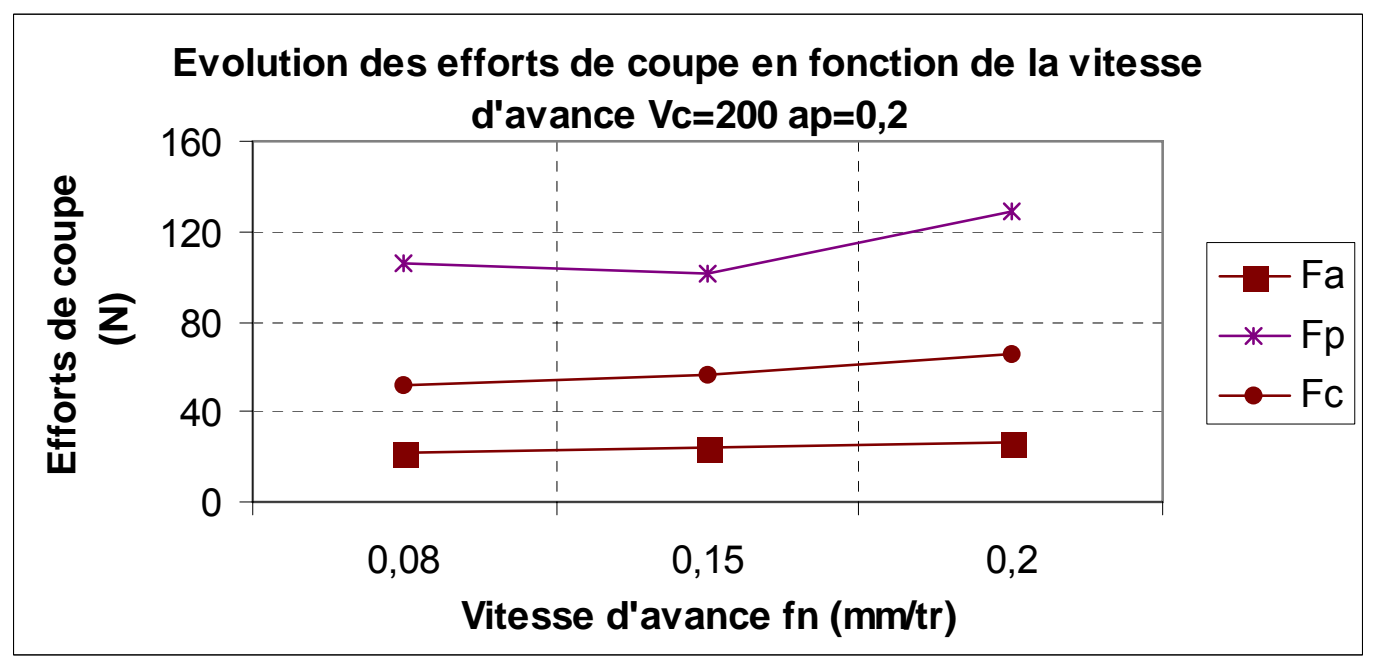

Figure 65 : Evolution des efforts de coupe en fonction de $f$

Le graphique ci-dessous montre les résultats de l'analyse statistique des effets de l'avance sur les efforts de coupe. L'effet de l'avance $f$ est monotone et croissant sur les trois composantes de l'effort de coupe.
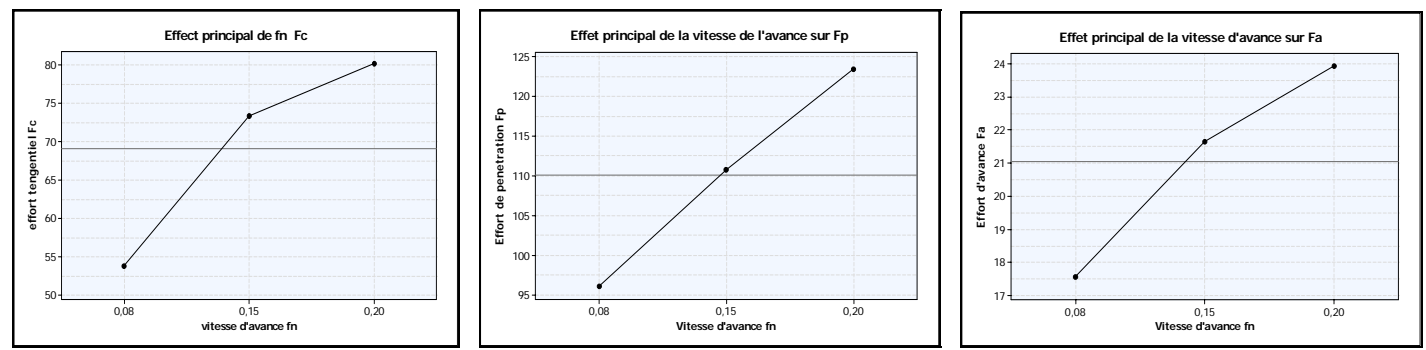

Figure 66 : Graphiques des effets de l'avance sur les efforts de coupe

\subsubsection{Evolution des efforts de coupe en fonction de profondeur de passe}

Le graphique de la figure 66 montre l'évolution des efforts de coupe en fonction de la profondeur de passe $a p$. Cette évolution est monotone, croissante pour les trois efforts $(F p, F c$ et $F a)$, mais l'effort 
de pénétration est le plus sensible à la variation de $a_{p}$. Cette augmentation est provoquée par l'accroissement de la section du copeau qui engendre l'augmentation du chargement de l'outil.

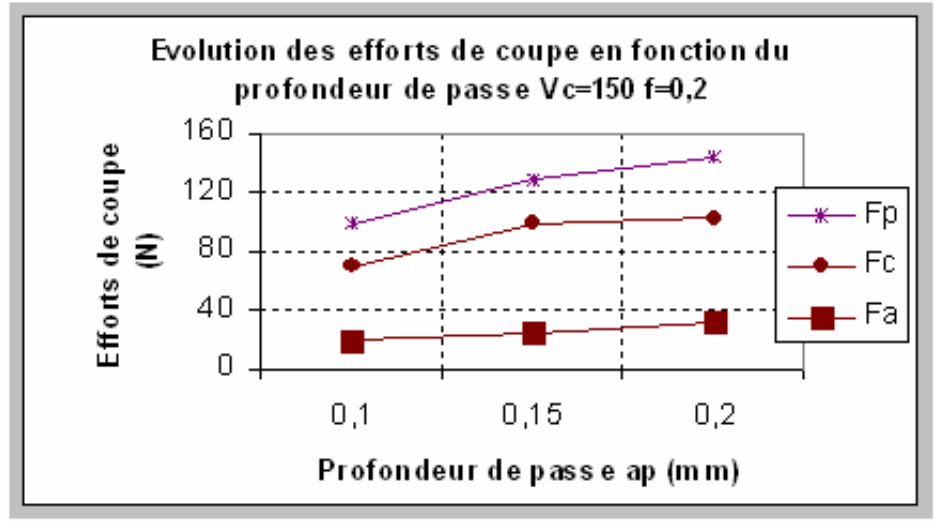

Figure 67 : Evolution des efforts de coupe en fonction de ap

Le graphique ci-dessous montre que les efforts de pénétration $F p$ et de coupe $F C$ sont les plus influencés par l'augmentation de la profondeur de passe alors que l'effort d'avance $F a$ est faiblement sensible à cette augmentation.

L'effort de pénétration est toujours le plus important en tournage dur. Cela s'explique par la géométrie négative de l'arête de coupe (chanfrein de $-20^{\circ}$ ).
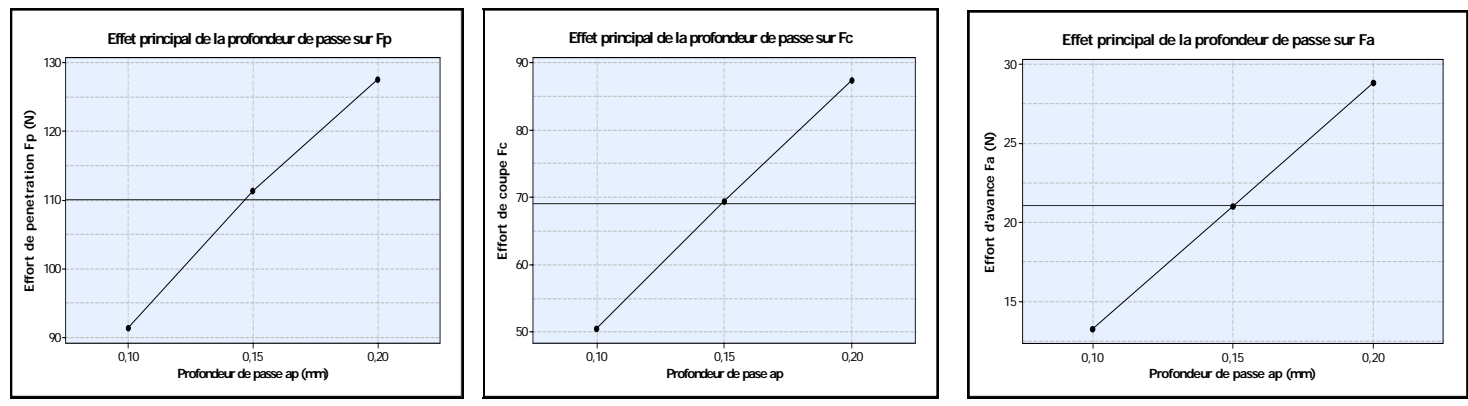

Figure 68 : Graphes des effets de la profondeur de passe sur les efforts de coupe

Le choix de valeurs faibles de la profondeur de passe est déconseillé, malgré les faibles efforts de coupe obtenus, parce que ces valeurs entraînent des pressions spécifiques de coupe énormes (de 8 à $10 \mathrm{Gpa}$ pour $V c=150 \mathrm{~m} / \mathrm{min}$ et $a p=0.05 \mathrm{~mm}$ ). L'augmentation de la profondeur de passe induit des sévères conditions de coupe. En effet, l'augmentation de la surface de coupe génère une élévation de la température qui accélère l'usure de l'outil, d'où la nécessité d'optimiser les conditions de coupe.

\subsubsection{Prédiction des efforts en fonction des paramètres de coupe}

Cette partie a pour objectif de modéliser les composantes de l'effort de coupe en fonction des paramètres d'usinage. Un modèle sous forme exponentielle qui à montré une fiabilité de prédiction de $R a$, peut s'écrire sous la forme suivante :

$$
F_{i}=C_{i}^{1} \cdot V_{c}^{c_{i}^{2}} \cdot f^{c_{i}^{3}} a_{p}^{c_{i}^{4}}
$$

Avec $i \in\{a, c, p\}$ : indice des composantes de l'effort de coupe.

$$
C_{i}^{1}, C_{i}^{2}, C_{i}^{3} \text { et } C_{i}^{4} \text { : Constantes du modèle. }
$$

Apres avoir appliqué la régression linéaire des résultats expérimentaux des différentes composantes de l'effort de coupe, on obtient les modèles suivants : 


\begin{tabular}{|c|c|}
\hline & Coefficient de détermination \\
\hline$F a=3677 . V_{c}^{-0.426} f^{0.413} a_{p}^{1.18}$ & $87.1 \%$ \\
\hline$F p=2416 . V_{c}^{-0.324} f^{0.274} a_{p}^{0.49}$ & $87.8 \%$ \\
\hline$F C=73130 . V_{c}^{-0.92} f^{0.44} a_{p}^{0.82}$ & $83.9 \%$ \\
\hline
\end{tabular}

Ces modèles permettent de prédire les valeurs des différentes composantes de l'effort de coupe.

Les graphiques ci-après représentent les histogrammes des valeurs expérimentales des efforts et celles obtenues par les modèles de prédiction. Ces graphiques affirment que ces modèles s'ajustent parfaitement avec les valeurs expérimentales.

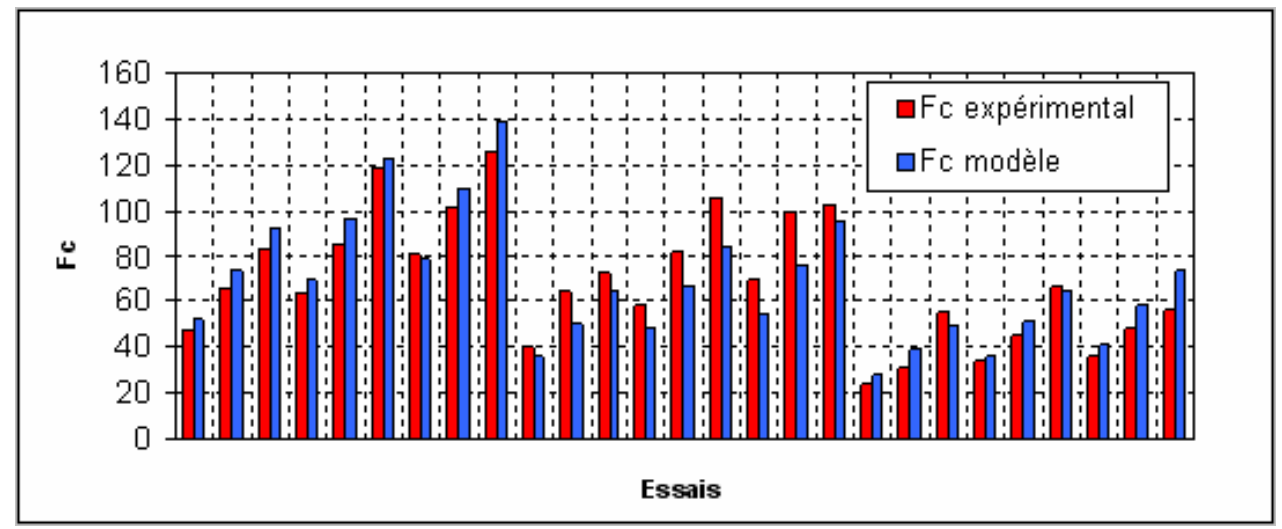

Figure 69 : Comparaison entre Fc calculé et celui donné par l' expérience.

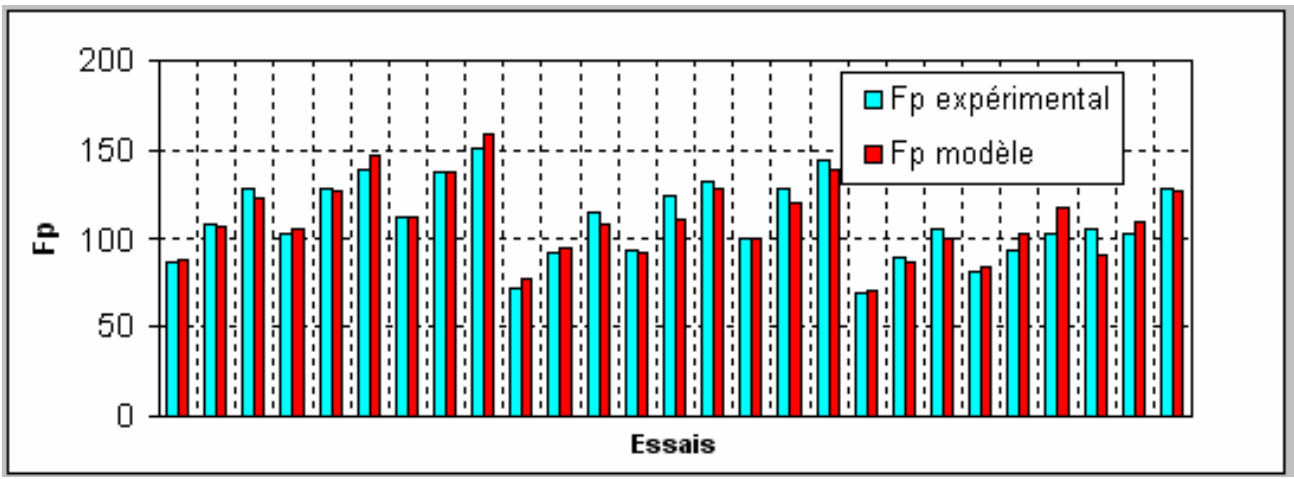

Figure 70 : Comparaison entre Fp calculé et celui donné par l' expérience.

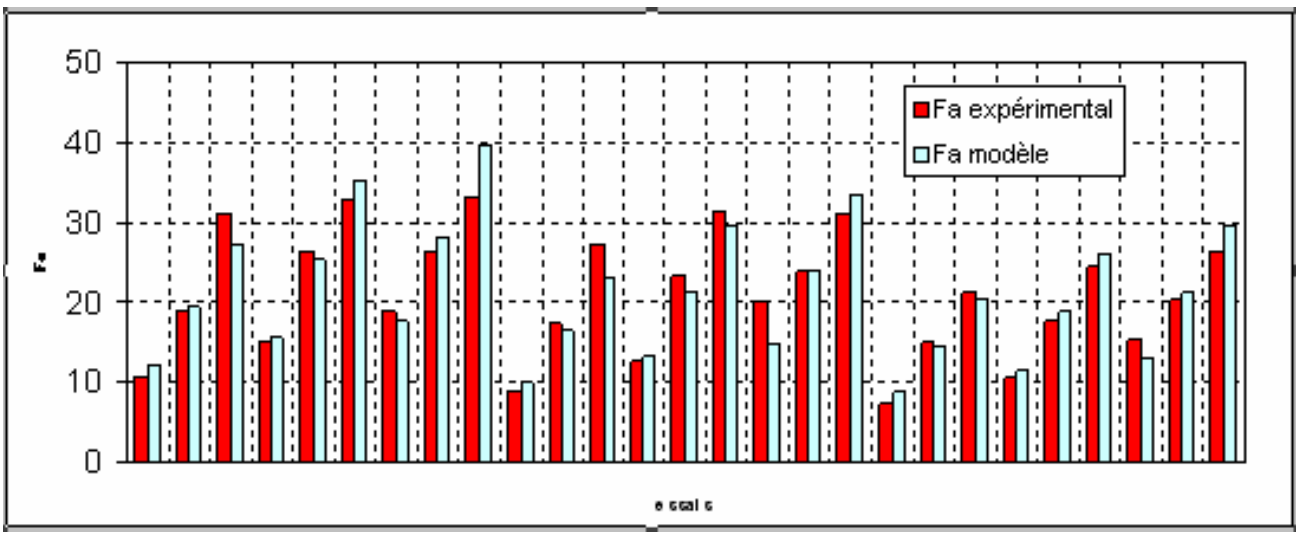

Figure 71 : Comparaison entre Fa calculé et celui donné par l'expérience. 


\subsubsection{Etude de l'usure de l'outil de coupe}

Dans cette partie, on s'intéresse à l'étude de :

- l'évolution de l'usure en fonction des paramètres de coupe,

- l'effet de l'usure sur l'évolution des efforts et de la rugosité de la pièce

- l'analyse des mécanismes et formes de l'usure en tournage dur.

\subsubsection{Evolution de l'usure}

Le graphique suivant illustre l'évolution de l'usure au cours du temps pour $V c=120 \mathrm{~m} / \mathrm{mn} f=0.1$ $\mathrm{mm} / \mathrm{tr}$ et $a p=0.2$. L'allure de la courbe est typique, elle est caractérisée par une zone de rodage (jusqu'à une longueur usinée de $0.5 \mathrm{~m}$ ) suivie par une zone de stabilisation (évolution constante : Zone 1), au cours de laquelle l'usure en dépouille évolue faiblement Zone 2. Par la suite, l'usure en cratère devient plus dominante, sur le chanfrein de l'arête Zone 3. Ensuite, l'outil se trouve dans une usure catastrophique, son évolution est rapide. Si l'usinage est prolongé, on arrive dans tous les cas à un délaminage et un écaillage du CBN.

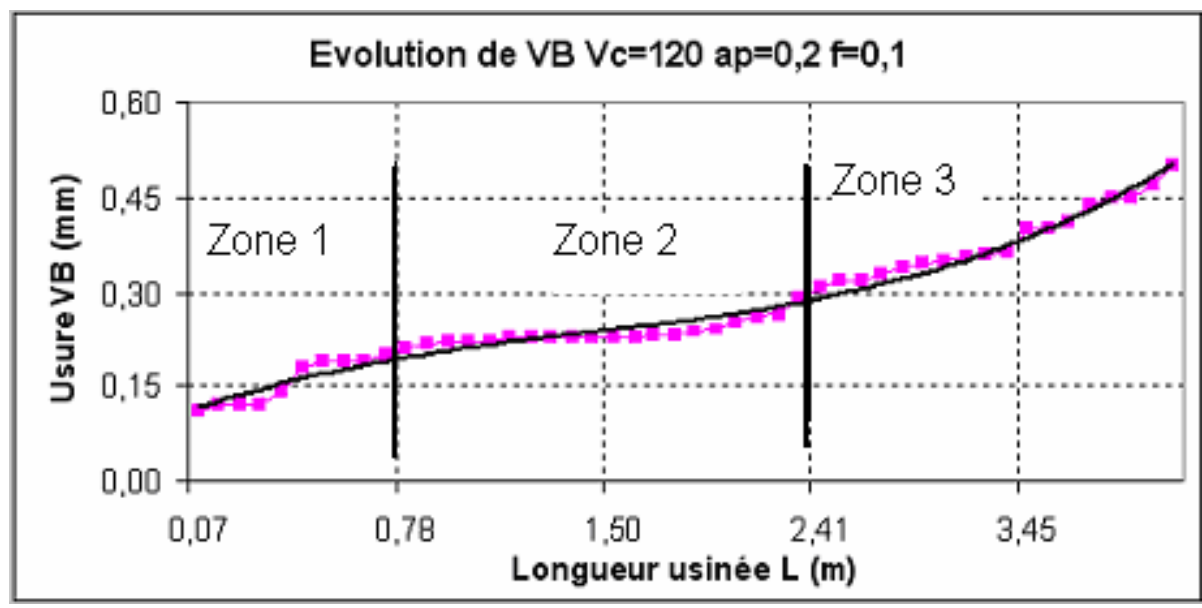

Figure 72 : Evolution de l'usure en fonction de la longueur usinée

L'influence des paramètres de coupe est significative sur l'usure. En effet, lors des essais, nous avons observé une nette évolution de l'usure en augmentant la vitesse de coupe. En effet, pour des vitesses de coupe élevées, on obtient de grandes vitesses de glissement copeau/outils qui vont entraîner une élévation importante de la température et une évolution rapide de l'usure.

De la même manière, l'influence de l'avance est importante. Les essais réalisés ont montré également une diminution rapide de la durée de vie de l'outil avec l'augmentation de l'avance.

\subsubsection{Effets de l'usure sur l'effort de coupe}

Le graphique ci-dessous montre l'évolution des efforts de coupe en fonction de l'usure de l'arête de coupe pour les conditions de coupe $V c=180 \mathrm{~m} / \mathrm{mn}, f=0,1 \mathrm{~mm} / \mathrm{tr}$ et $a p=0,2 \mathrm{~mm}$.

Les efforts augmentent en fonction du temps d'usinage, ceci est dû à l'évolution de l'usure qui augmente les surfaces de contact outil pièce et par conséquent engendre des efforts de frottement qui s'additionnent aux efforts induits par la coupe.

L'étude de l'allure de l'évolution de ces efforts montre qu'ils passent par une période de faible durée dont l'évolution est rapide ( $\mathrm{VB}<0.2 \mathrm{~mm}$ ). L'observation de l'usure durant cette période montre que celle en dépouille est prépondérante, alors que celle en cratère est faible.

Ensuite, les efforts de coupe passent par une période $(0.2 \mathrm{~mm}<\mathrm{VB}<0.35 \mathrm{~mm})$ où les valeurs des trois composantes varient légèrement, les efforts de coupe et de pénétration $F C$ et $F p$ gardent respectivement les valeurs de $110 \mathrm{~N}$ et $120 \mathrm{~N}$. 
La troisième période est caractérisée par une évolution accentuée de l'usure. A partir d'une valeur d'usure $V B$ de $0.35 \mathrm{~mm}$, la vitesse d'évolution des composantes des efforts de coupe et de pénétration s'accroît rapidement, les efforts de coupe et de pénétration passent respectivement à 200 et $220 \mathrm{~N}$ pour $\mathrm{VB}=0.84 \mathrm{~mm}$, l'observation de l'usure sur la plaquette montre une évolution rapide et un changement de la géométrie de l'arête de coupe (le chanfrein est totalement éliminé), un écaillage se présente sur la face d'attaque et celle en dépouille. L'évolution de l'effort d'avance reste relativement faible. (voir photos ci-dessous).

L'allure des courbes de l'évolution des efforts de coupe, montre la sensibilité de l'effort de pénétration $F p$ et de coupe $F C$ à l'usure. Ceci implique que le suivi de l'évolution de ces efforts peut nous permettre de surveiller l'évolution de l'usure.

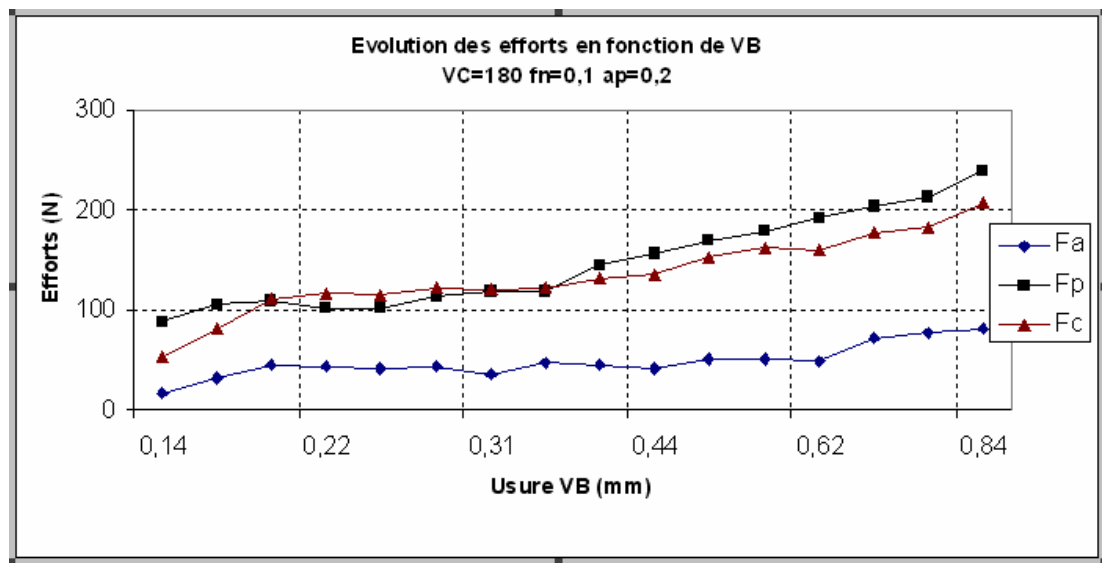

Figure 73 : Evolution des efforts de coupe en fonction de l'usure et effets de l'usure sur la rugosité

Le profil théorique de l'état de la surface en tournage est déterminé par l'avance $f$ et du rayon de la pointe de l'outil $r_{\varepsilon}$. Ce profil est la conjugaison de l'effet de la géométrie de l'arête et des conditions d'usinage. Mais en pratique, l'usure a un effet sur la rugosité de la surface usinée.

L'étude de l'évolution de la rugosité de la surface usinée en fonction de la longueur d'usinage (figure 73) montre que la rugosité de la surface n'est plus constante, mais elle se dégrade au cours du temps. Cette dégradation de la rugosité est attribuée à l'évolution de l'usure.

L'analyse de la courbe d'évolution de la rugosité en fonction de l'usure en dépouille montre l'influence de l'usure sur la qualité d'usinage. L'état de la surface se dégrade d'une façon croissante avec la progression de l'usure $V B$ pour atteindre une dégradation rapide et accentuée à partir de $V B=0.3 \mathrm{~mm}$. Après ce seuil, la vitesse de dégradation de la rugosité devient très rapide.

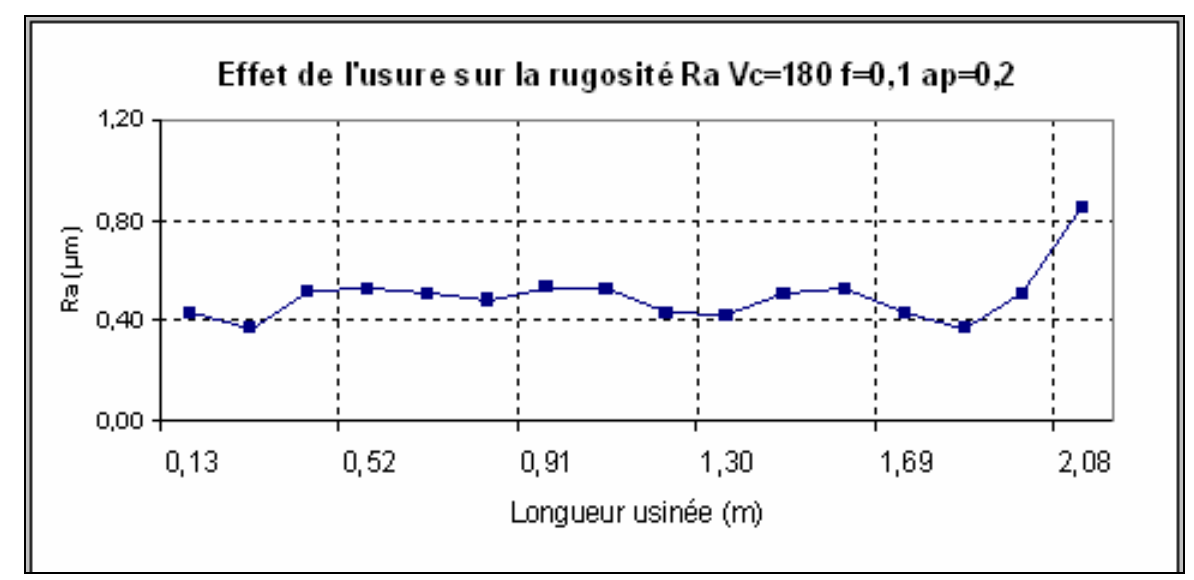

Figure 74 : Effet de l'usure sur l'évolution de la rugosité de la surface usinée 


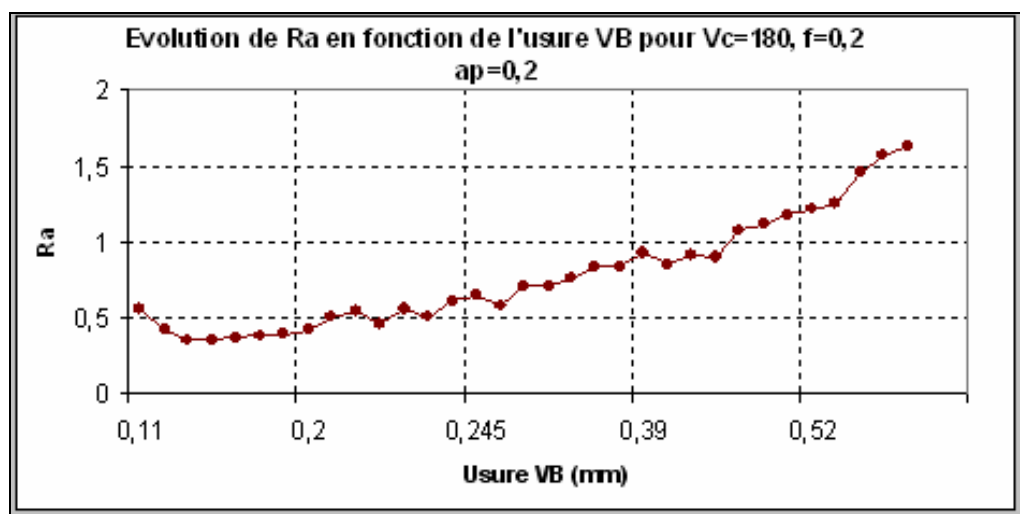

Figure 75 : Evolution de Ra en fonction de l'usure VB pour $V c=180 \mathrm{~m} / \mathrm{mn}, f=0.2 \mathrm{~mm} / \mathrm{tr}$ et ap $=0.2 \mathrm{~mm}$

\subsubsection{Analyse de la morphologie de l'usure}

Vu la géométrie de l'arête de coupe en tournage dur, la forme de l'usure des outils de coupe n'est pas uniforme et elle est liée étroitement à l'épaisseur du copeau le long du contact outil / pièce. Ceci est la conséquence de la répartition des contraintes au niveau de l'arête de coupe.

En comparant la géométrie du copeau à la morphologie d'usure, on peut conclure que la zone la plus sévère de l'usure en cratère est celle qui a la valeur d'épaisseur de copeau maximale, c'est celle qui subit les contraintes mécanique et thermique les plus élevées par rapport aux autres zones.

L'analyse et le suivi de l'usure au cours des essais réalisés prouvent que l'écaillage et la rupture de l'arête se produisent au niveau de la zone dont l'épaisseur du copeau est maximale.

La figure 76 ci-dessous illustre cette constatation et montre la distribution des contraintes sur l'arête de coupe. Pour mieux analyser la forme de l'usure, il paraît intéressant d'étudier la répartition des contraintes thermiques et mécaniques au niveau de la zone de contact pièce / outil.

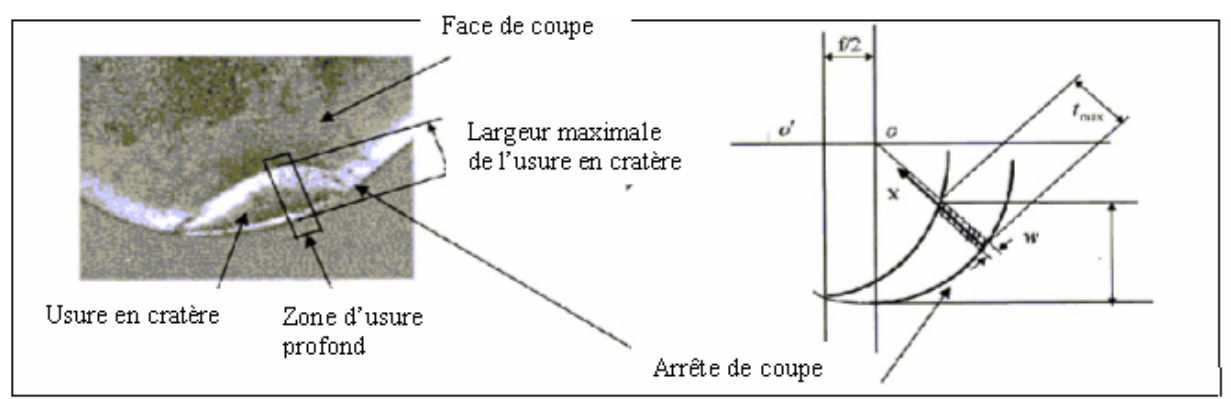

Figure 76 : Morphologie de l'usure en cratère [Huang 03]

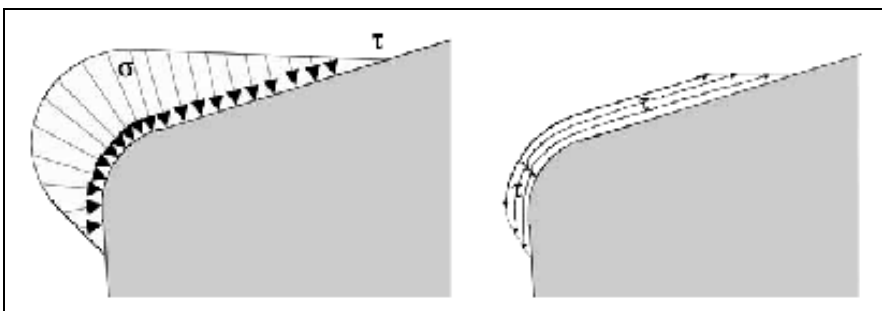

Figure 77 : Répartition des contraintes sur la pointe de l'outil

Les photos suivantes (figure 78) qui suivent montrent des états d'usure issus des essais d'usure réalisés. La figure (a) montre l'usure en cratère. Sa forme est liée aux spécifications géométriques de la coupe en tournage dur. On constate que le chanfrein de l'arête de coupe a totalement fondu comme conséquence de l'usure en cratère. La figure (b) représente l'écaillage de la face de coupe qui est le résultat d'une usure accentuée et des sollicitations importantes sur la pointe de l'outil. La figure (c) représente l'usure en dépouille. Il est nettement clair que l'abrasion est la cause principale de l'usure ; 
les sillons sont le fait de l'abrasion de la face de l'outil par les particules dures de la pièce. L'usure en cratère est représentée par la figure (a). La figure (d) montre l'endommagement total de l'arête de coupe par l'association de l'usure en cratère et en dépouille.

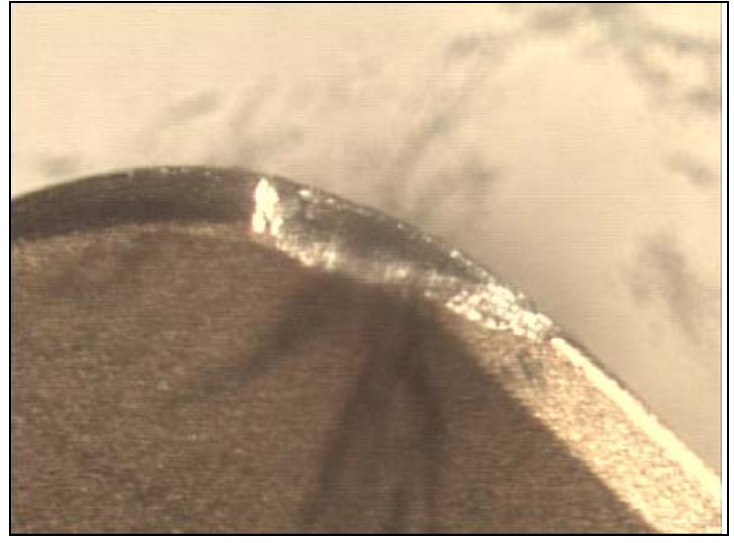

(a) Usure en cratère

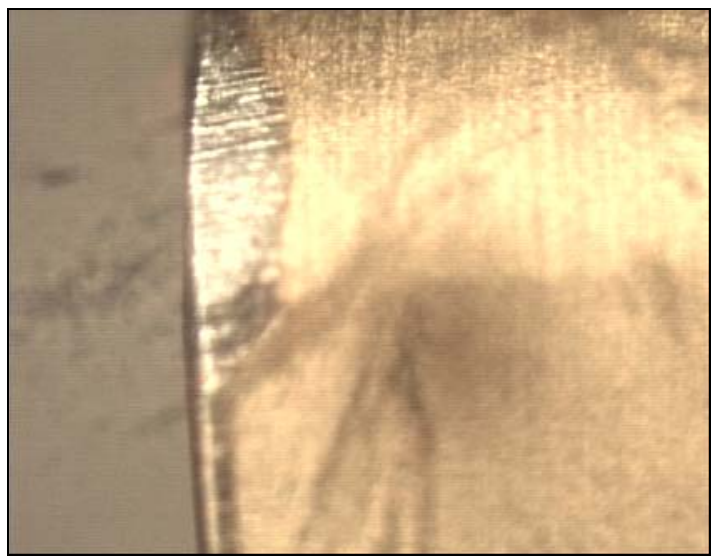

(c) Usure en dépouille

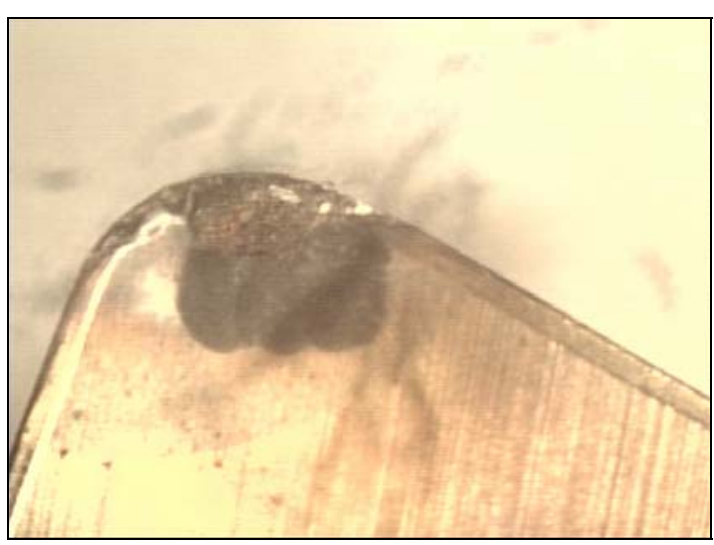

(b) Ecaillage de l'arête de coupe

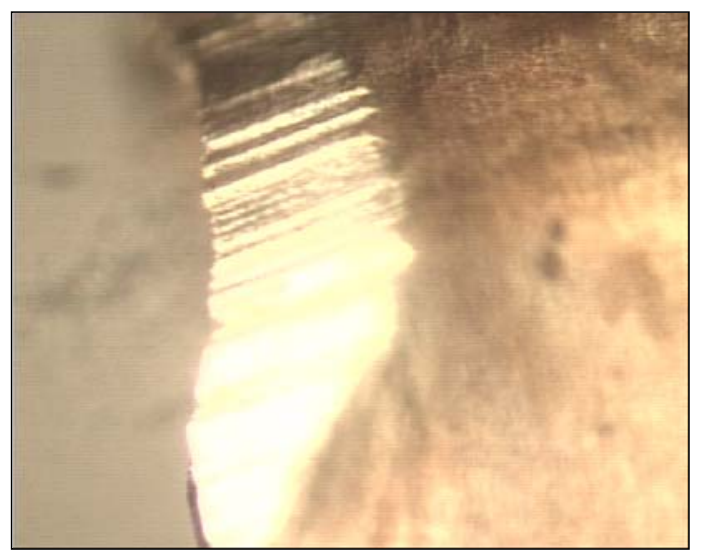

(d) Usure en dépouille de valeur élevée Figure 78 : Photos de l'usure de l'outil de coupe (agrandissement 60 fois)

\subsection{Simulation numérique du comportement de l'outil}

Cette partie est une synthèse de l'étude de la numérique du comportement de l'outil lors de l'usinage. Nous avons mené cette étude en vue de déterminer l'effet du mouvement de l'outil sur la qualité de la surface en tournage dur à partir des résultats obtenus dans la partie expérimentale.

\subsubsection{Etude du déplacement de l’outil}

Cette étude numérique est effectuée à l'aide du logiciel Abaqus afin de déterminer l'impact de la vibration relative outil/pièce sur la rugosité de la surface obtenue en tournage dur. Cette étude consiste à déterminer le déplacement relatif de l'outil sous l'effet des efforts de coupe appliqués sur l'outil, ces efforts sont les résultats des expériences effectuées.

\subsubsection{Modèle de simulation numérique (Hypothèses)}

Cette étude numérique est réalisée par le logiciel de calcul par éléments finis Abaqus. A partir du modèle CAO de l'outil, on a réalisé un maillage volumique. Le chargement mécanique ponctuel sur la pointe de l'outil est obtenu à partir des efforts de coupe mesurés par le banc de mesure Kistler, lors des essais réalisés durant l'étude expérimentale. La variation des efforts de coupe au cours du temps est prise en compte. Ces efforts sont introduits dans le logiciel sous forme d'une fonction du temps.

La plaquette est considérée comme un corps rigide afin de dissocier l'effet de sa déformation de celui du corps de l'outil. 
L'outil est encastré par deux faces sur la tourelle. Le reste du système usinant n'est pas pris en considération dans cette étude.

La modélisation de l'outil est présentée dans la figure suivante

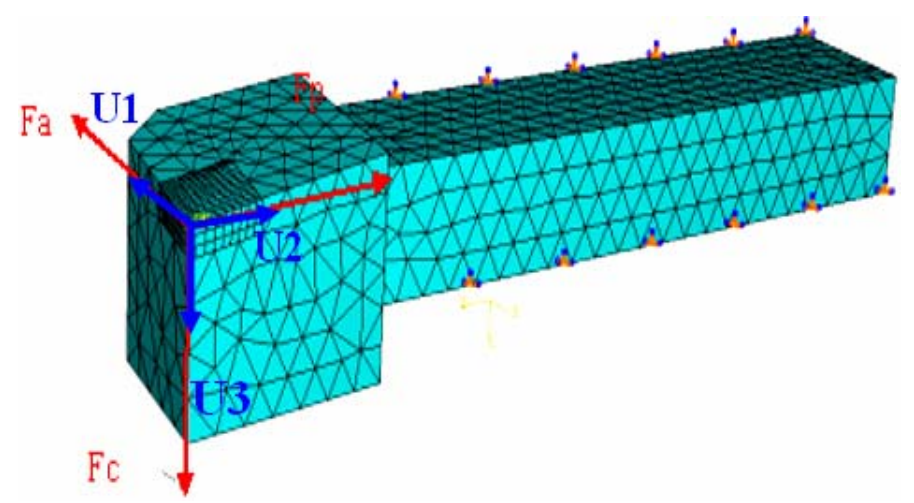

Figure 79 : Modèle éléments finis de l'outil

Dans l'objectif de comprendre le phénomène de déplacement de l'outil sous l'effet de différentes interactions du système usinant, on a considéré les efforts de coupe obtenus dans de diverses conditions, ces paramètres sont présentés ci-après :

\begin{tabular}{|l|c|c|}
\hline$V_{c}(\mathrm{~m} / \mathrm{mn})$ & 180 & 120 \\
\hline$f(\mathrm{~mm} / \mathrm{tr})$ & 0,1 & 0,2 \\
\hline
\end{tabular}

\subsubsection{Résultats interprétations et discussions}

Les résultats obtenus de ce calcul sont les déplacements de la pointe de l'outil selon les trois axes. La figure ci-dessous présente un exemple des courbes de déplacements de l'outil au cours du temps, U1 représente le déplacement axial de l'outil, U2 le déplacement radial, U3 représente le déplacement tangentiel et U représente l'amplitude des déplacements.

Ces déplacements sont les effets des efforts de coupe. Les fluctuations dans ces courbes sont l'effet de la variation des efforts au cours du temps.

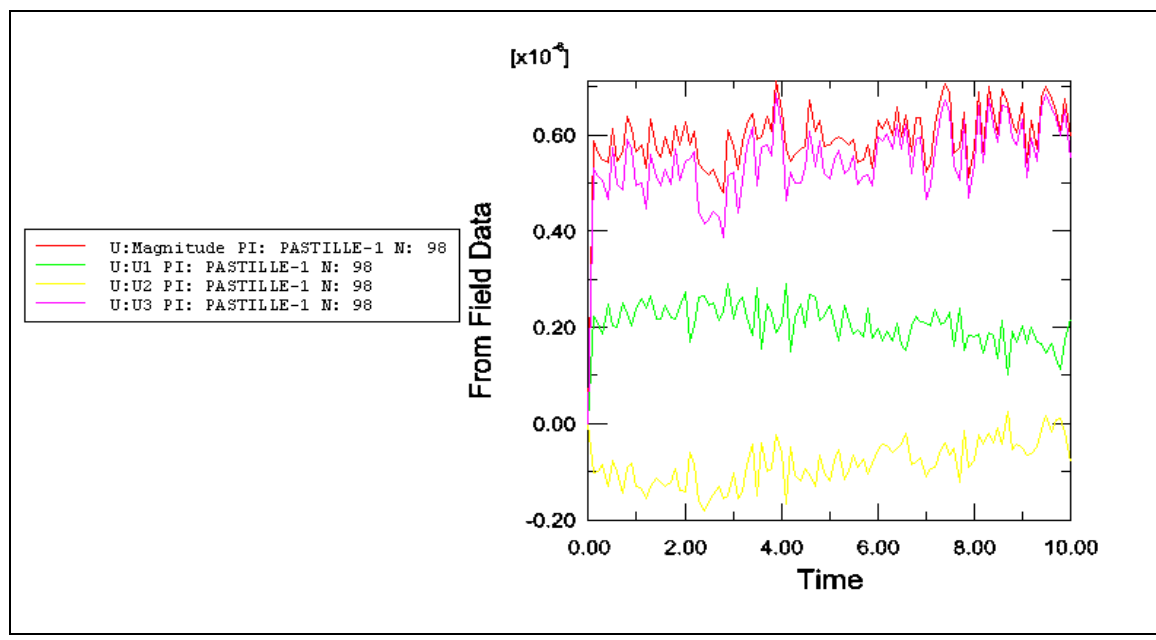

Figure 80 : Amplitude des déplacements de la pointe de l'outil pour $V_{c}=180 f=0.2$ ap $=0.2$

Une présentation graphique des déplacements peut être générée par Abaqus sous forme d'une cartographie afin de mieux analyser les résultats. 


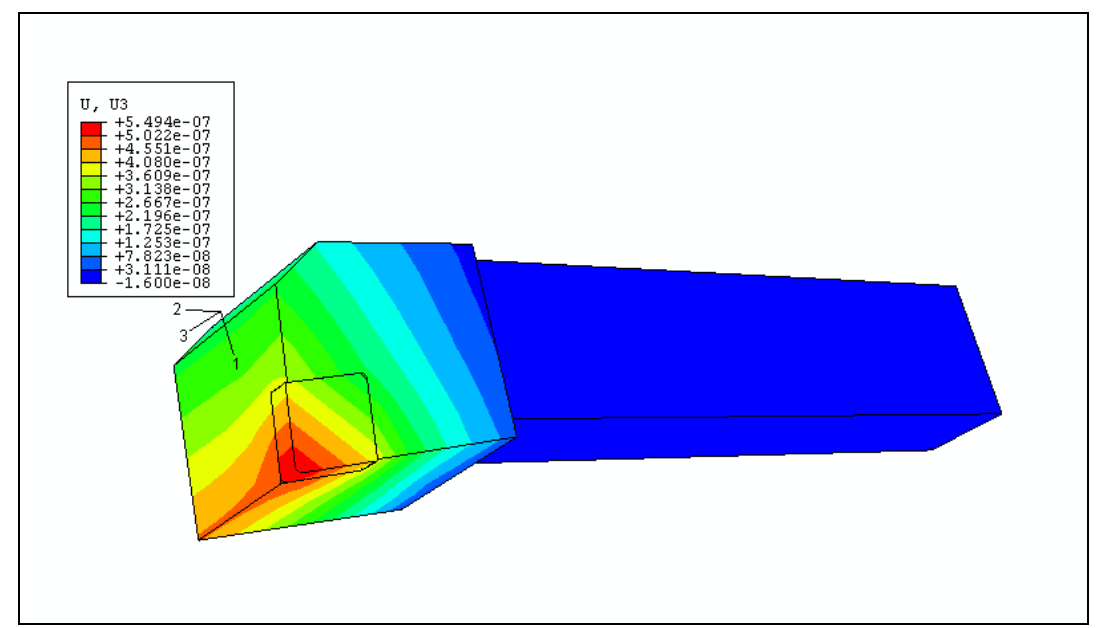

Figure 81 : Cartographie du Déplacement tangentiel de l'outil $V_{C}=180 \mathrm{~m} / \mathrm{mn} f=0.2 \mathrm{tr} / \mathrm{mn}$

Les résultats obtenus par cette étude numérique sont recueillis sous forme de fichier texte et traités par Excel pour être présentés sous forme de courbes.

La première remarque qu'on peut tirer de ces courbes est la fluctuation des déplacements. Les chargements de l'outil (efforts) sont à l'origine de ces fluctuations.

Les variations observées sur ces courbes sont plus importantes pour U3 (déplacement tangentiel), et elles croissent avec l'augmentation de la vitesse de coupe et de l'avance.

L'augmentation de la vitesse de coupe de 120 à $180 \mathrm{~m} / \mathrm{mn}$ présente un effet décroissant et faible sur l'amplitude du déplacement (figure ci-dessous).

Ces constatations prouvent que l'usinage est plus stable avec l'augmentation de la vitesse de coupe et la diminution de l'avance.

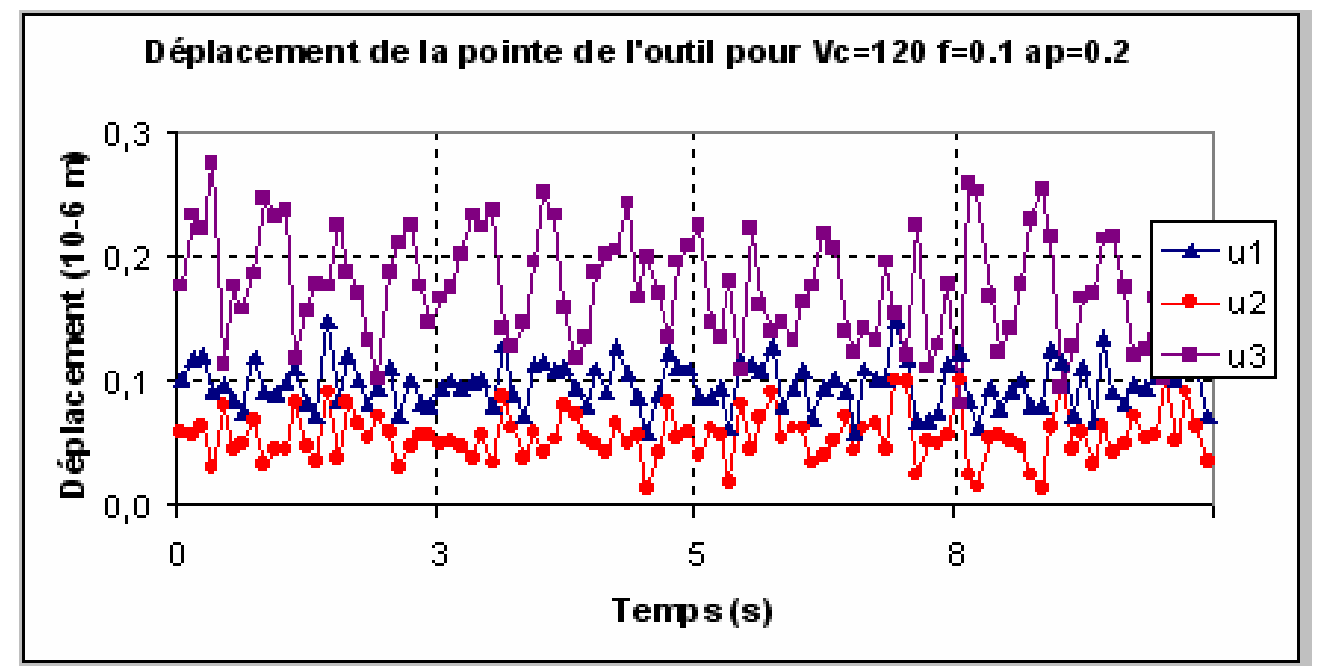

Figure 82 : Amplitude des déplacements de la pointe de l'outil pour $V_{c}=120, f=0.1$ et ap $=0.2$

Le tableau 4 ci-dessous contient un récapitulatif de la moyenne des déplacements suivant les trois directions (U1, U2, et U3) ainsi que l'amplitude U.

\begin{tabular}{|c|c|c|c|c|c|}
\hline $\boldsymbol{V} \boldsymbol{c}(\mathbf{m} / \mathbf{m n})$ & $\boldsymbol{f} \mathbf{\text { tr} / \mathbf { m n }}$ & $\mathbf{U}$ & $\mathbf{U 1}$ & $\mathbf{U} 2$ & $\mathbf{U 3}$ \\
\hline 120 & 0,1 & 0,208 & 0,096 & 0,055 & 0,173 \\
\hline 120 & 0,2 & 0,663 & 0,205 & 0,049 & 0,625 \\
\hline 180 & 0,1 & 0,219 & 0,139 & 0,113 & 0,101 \\
\hline 180 & 0,2 & 0,603 & 0,201 & 0,076 & 0,559 \\
\hline
\end{tabular}

Tableau 4 : Amplitudes moyennes des déplacements pour les divers paramètres de coupe. 


\subsubsection{Effets des paramètres de coupe sur l'amplitude des déplacements}

L'amplitude des déplacements suivant la direction tangentielle est toujours supérieure à celle dans la direction axiale et radiale. Pour des conditions de coupe $V C=180 \mathrm{~m} / \mathrm{mn}$ et $f=0.2 \mathrm{~m} / \mathrm{mn}$, le déplacement tangentiel est en moyenne trois fois, supérieur à celui suivant la direction axiale, et six fois, supérieur à celui suivant la direction radiale. Ces constatations nous permettent de conclure que les déplacements de la pointe de l'outil sont dus essentiellement à la flexion globale de l'outil dans la direction tangentielle et par la suite il se retire de la pièce suivant la direction radiale.

Les résultats obtenus (Tableau 4) montrent que l'amplitude du déplacement est influencée par l'avance, pour une vitesse de coupe de $120 \mathrm{~m} / \mathrm{mn}$ l'amplitude des déplacements varie de $0.208 \mu \mathrm{m}$ (pour une avance de $0.1 \mathrm{~mm} / \mathrm{tr}$ ) à $0.663 \mu \mathrm{m}$ (pour une avance $0.2 \mathrm{~mm} / \mathrm{tr}$ ).

L'effet de l'avance est considérable. En effet, l'amplitude U des déplacements atteint des valeurs faibles pour une avance de $0.1 \mathrm{~mm} / \mathrm{tr}$.

L'effet de la vitesse de coupe sur le déplacement est plus faible par rapport à celui de l'avance, les amplitudes des déplacements diminuent avec l'accroissement de la vitesse de coupe. Ceci peut être expliqué par la diminution de l'énergie nécessaire à la formation du copeau suite à l'adoucissement du matériau de la pièce.

Pour mieux analyser l'effet des paramètres de coupe (vitesse de coupe et l'avance), nous avons mené une analyse statistique sur Minitab14. Les résultats de cette analyse sont représentés dans les graphiques suivants.
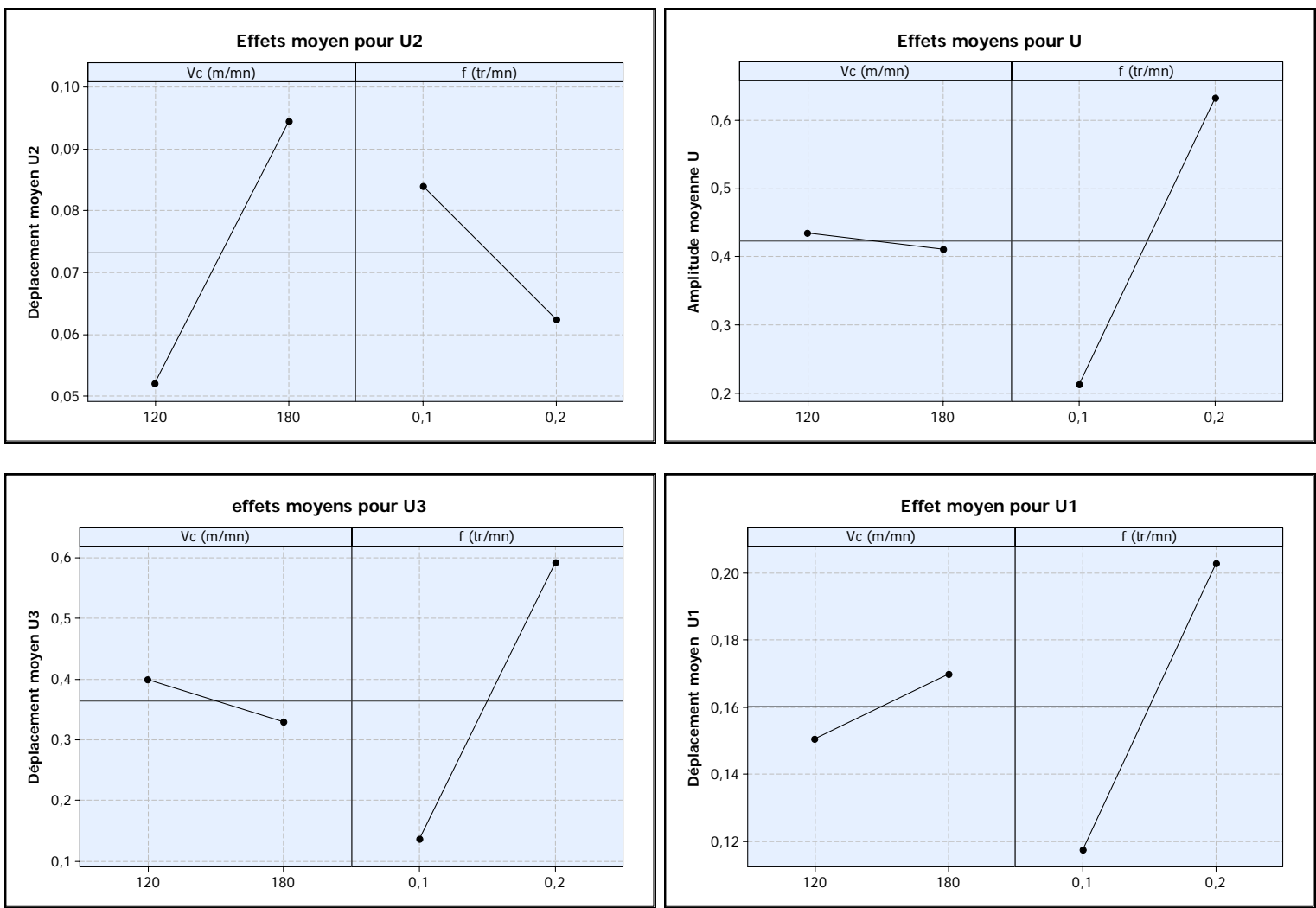

Figure 83 : Effets des paramètres de coupe sur les amplitudes des déplacements

Ces graphiques montrent que l'avance a l'effet le plus important sur les déplacements. L'effet de l'avance sur U3 passe de $0,15 \mu \mathrm{m}$ à $0,57 \mu \mathrm{m}$ lorsque $f$ varie de 0,1 à $0,2 \mathrm{~mm} / \mathrm{tr}$. La composante du déplacement $\mathrm{U} 1$ est affectée par la variation de $\mathrm{f}$, une variation de $f$ de 0,1 à 0,2 engendre une augmentation de l'effet sur U1 de 0.08 à $0,22 \mu \mathrm{m}$. 
La vitesse de coupe a un effet moyen moins important que celui de l'avance. Lorsque $V c$ passe de 120 à $180 \mathrm{~m} / \mathrm{mn}$, l'effet de $V c$ sur U3 passe de 0,4 à $0.32 \mu \mathrm{m}$.

Les valeurs des déplacements et les effets de $V c$ et $f$ suivant la direction U2 sont faibles par rapport aux autres directions.

La figure suivante représente l'évolution du déplacement axial U1 en fonction de la vitesse de coupe $V c$ et de l'avance $f$. On constate que l'amplitude de U1 est la plus faible pour de faibles valeurs de $f$ et de $V c$.

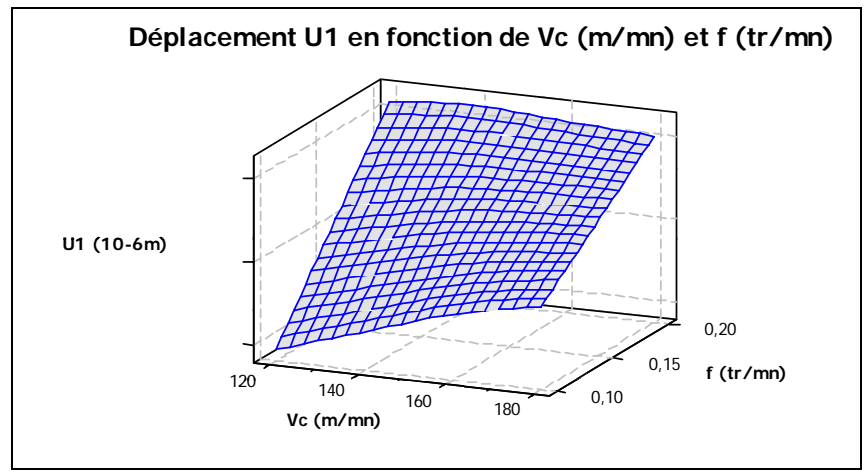

Figure 84 : Evolution du déplacement axial en fonction de Vc et $f$.

La variation de la valeur du déplacement $\mathrm{U} 2$ en fonction des paramètres de coupe est illustrée dans la figure suivante. L'effet de la vitesse de coupe est croissant alors que celui de l'avance est décroissant.

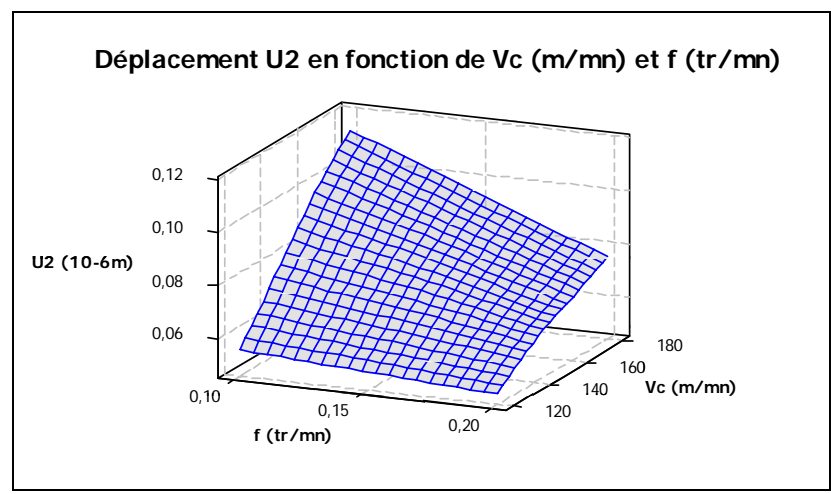

Figure 85 : Evolution du déplacement radial en fonction de Vc et $f$

La figure suivante montre l'évolution du déplacement tangentiel en fonction de $V c$ et $f$. L'effet de la variation de $f$ sur l'amplitude U3 est la plus important.

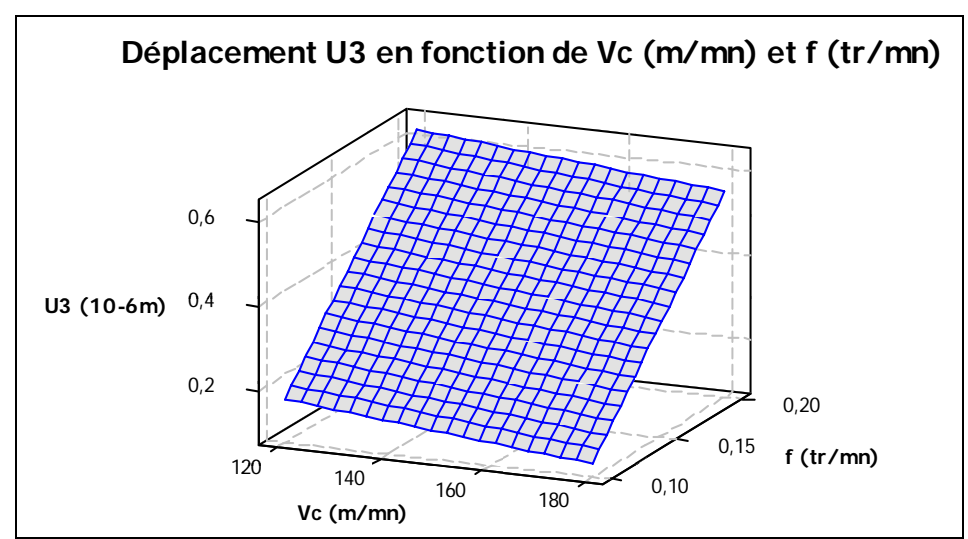

Figure 86 : Evolution du déplacement tangentiel en fonction de Vc et $f$. 
Suite à cette étude nous avons constaté que l'outil de coupe se déforme sous l'action des efforts de coupe. La pointe fléchissant suivant la direction axiale et tangentielle, est comprimée dans la direction radiale. Par conséquent, la pointe se retire de la pièce, ce qui modifie l'allure théorique de la surface. La composante tangentielle est la plus dominante, c'est elle qui affecte le plus, la rugosité de la surface.

Pour une vitesse de coupe de $180 \mathrm{~m} / \mathrm{mn}$, une avance de $0.2 \mathrm{~mm} / \mathrm{tr}$ et une profondeur de passe de $0.2 \mathrm{~mm}$, la rugosité arithmétique moyenne mesurée de la surface est de $0.32 \mu \mathrm{m}$ alors que l'amplitude du déplacement de l'outil selon la direction tangentielle U3 est de 0,18. Ceci montre que le déplacement de l'outil a un effet dans la génération du profil réel de la surface.

Le profil de la surface est le résultat de la superposition du profil théorique déterminé par les conditions de coupe et la géométrie de la pointe de l'outil et du mouvement relatif outil-pièce dû aux effets de la dynamique de la machine.

\subsection{Retombées scientifiques des approches proposées.}

Ce travail a fait l'objet d'un Mastère en Génie Mécanique au sein du Laboratoire de Génie Mécanique de l'Ecole Nationale d'Ingénieurs de Monastir sous mon encadrement scientifique (Voir annexe II, Fiches d'Encadrement Scientifique : Mastère de Mr. Imed Hajjaji).

Ce travail a abouti à une communication internationale de type Poster. (voir Annexe I : Fiches Publications Scientifique). D'autres publications sont en cours de rédaction.

\section{Communication Nationale :}

- I. Hajjaji, A. BenAmara, R. Fredj «Etude Expérimentale de l'influence des paramètres de coupe sur l'état de surface en tournage dur » Premier Congrès International Conception et Modélisation des Systèmes Mécaniques : (Poster) CMSM2005; 23-25 Mars 2005; Hammamet - Tunisie. 


\section{Modèle de conception de moules d'injection plastique basé sur les Features}

Ce travail s'inscrit dans le cadre de la modélisation par les features qui constitue la base des systèmes de $\mathrm{CAO}$ actuels. Le modèle proposé permet de concevoir des plaques empreintes des moules d'injection plastique en tenant compte dès la conception des contraintes de fabrication, au même titre que des contraintes fonctionnelles.

En effet, les opérations d'enlèvement de la matière sont représentées par des opérations topologiques de soustraction, les entités (formes + information technologiques) correspondent, le plus souvent, à des opérations spécifiques au procédé de fabrication utilisé.

Nous avons voulu appliquer ce modèle au domaine du moulage par injection des pièces en matière plastique. L'intérêt accordé à ce domaine de conception de moule est justifié par le fait que :

- C'est un domaine qui reflète bien le gain de temps qu'on peut lui apporter, de par la complexité de son processus de conception-fabrication.

- C'est un domaine qui fait intervenir plusieurs acteurs lors du processus de conceptionfabrication : ce qui donne à la recherche l'opportunité de traiter du problème des points de vue multiples dans un environnement d'ingénierie concourante.

- Sa bonne représentativité du processus de conception (un moule est un produit souvent complexe : forme, liaisons, dimensionnement).

\subsection{Etat de l'art : Conception des moules d'injection plastiques basée sur les features}

La majorité des travaux de recherche confirme l'intérêt substantiel du concept feature. En effet, les features constituent un support des informations relevant de domaines divers et variés, ce qui leur confère une grande puissance d'intégration de plusieurs activités du cycle de vie d'un produit mécanique.

L'état de l'art dans ce domaine révèle trois tendances principales :

- Reconnaissance des features

- Conception par les features :

- Approche hybride :

L'approche de la reconnaissance des features est une approche qui vient pour extraire, à partir d'un modèle géométrique, tout ce qu'elle reconnaît comme features. Cette approche se heurte à des problèmes de reconnaissance et de compatibilité avec les différents systèmes de CAO. Notons que le problème majeur de cette approche est les interférences entre les features.

En contre partie, l'approche « feature modelling » ou conception par les features, est une approche dont l'objectif est de définir à la fois un support de conception permettant une intégration réelle des fonctions de conception et de fabrication en respectant les besoins des utilisateurs [Loriod 89].On adoptera dans notre cas cette approche; qui consiste en la création du modèle de produit par des opérations topologiques de soustraction ou d'addition successives sur une feature de base dite aussi feature primaire.

C'est une approche qui relie les deux approches précédentes, à savoir, la modélisation par les features et la reconnaissance des features.

La modélisation des moules d'injection plastique a fait l'objet de plusieurs travaux de recherche dont nous donnons un bref aperçu dans les paragraphies ci-dessous.

L'étude de Pratt [Pratt 93] a portée sur le développement d'une expertise pour l'évaluation de la « fabricabilité » de la pièce réalisée par injection plastique. Mais leur recherche a été menée en ne tenant compte que de l'aspect conception. 
Par contre, Lee. [Lee 96] a investigué des outils et logiciels pour supporter la Conception concourante Pour la Fabrication des Moules d'Injection CPFMI. Lee propose un noyau d'un système de fabrication (kernel) défini comme étant la source des informations de fabrication et du produit qui doit être fourni pour chaque application. Pour supporter la réalisation du produit, le modèle de produit fournit une source consistante d'informations sur celui-ci pendant la progression du processus de conception, tandis que le modèle de la fabrication saisit les informations relatives aux procédés et ressources de fabrication. Dans son développement, il a identifié le problème d'interactions entre, d'une part, le point de vue de la moulabilité « conception du produit à injecter », et d'autre part, les points de vue de la conception du noyau et de la cavité du moule d'injection.

Dans le cadre de leur recherche dans le domaine des systèmes d'aide à la conception, Ahmed Al Ashaab [Ashaab 94] et Lee [Lee 96] ont travaillé sur la conception des pièces moulées en matière plastique. Dans le cadre du projet MOSES, Al Ashaab [Ashaab 94] a développé le modèle de fabrication pour les pièces obtenues par moulage à injection. Ce modèle est une source commune d'information bien définie et structurée supportant la prise de décision en CPF des applications de moulage par injection. Son développement constitue la base de la représentation de la capabilité du procédé relative au domaine du moulage par injection.

\subsection{Features inhérentes au domaine de la conception des moules}

La conception par les features exige l'utilisation d'une bibliothèque de features prédéfinies. La tâche du concepteur mouliste consiste à instancier et valider ces features. Ci-dessous, nous présentons une sélection des features spécifique au domaine de la conception de moules d'injection plastique.

- feature primaire ou feature plaque empreinte (ou porte empreinte, c'est la plaque fournie normalement par un fournisseur d'éléments standards);

- feature empreinte femelle ou cavité (poche) ;

- feature empreinte male ou noyau (saillie);

- feature seuil d'alimentation (et le canal d'alimentation);

- feature évent ;

- feature trou lisse lamé ;

- feature trou lisse borgne ;

- feature trou lisse débouchant ;

- feature rainure en $\mathrm{T}$;

- feature rainure en $\mathrm{U}$;

La figure 87 illustre une feature « rainure en $U$ ».

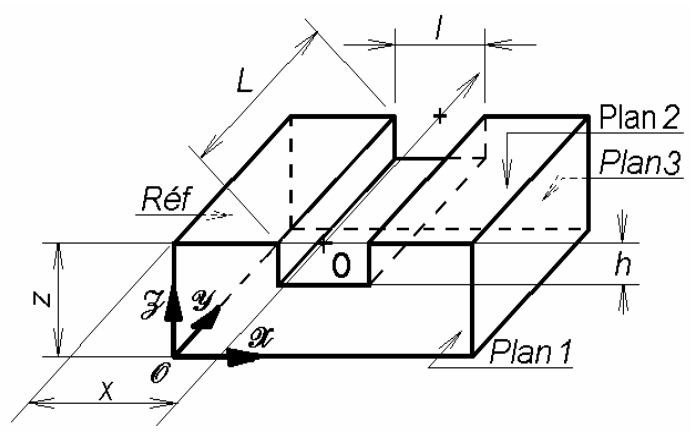

\begin{tabular}{|c|c|}
\hline \multicolumn{2}{|l|}{ DIMENSIONS } \\
\hline Largeur de la rainure & 1 \\
\hline Longueur de la rainure & $\mathrm{L}$ \\
\hline Profondeur de la rainure & $\mathrm{h}$ \\
\hline Position de la rainure par rapport à Réf & $\mathrm{X}, \mathrm{Z}$ \\
\hline Tolérance sur L & $\mathrm{T}_{\mathrm{L}}$ \\
\hline Tolérance sur 1 & $\mathrm{~T}_{1}$ \\
\hline Tolérance sur h & $\mathrm{T}_{\mathrm{h}}$ \\
\hline Etat de surface (frr-frb) & $0,8.25 \mu \mathrm{m}$ \\
\hline \multicolumn{2}{|l|}{ RELATIONS GEOMETRIQUES } \\
\hline Parallélisme & Réf \\
\hline Symétrie & Réf \\
\hline \multicolumn{2}{|l|}{ RELATIONS TOPOLOGIQUES } \\
\hline Débouche sur & plan 2 - plan 3 \\
\hline Commence sur & plan 1 \\
\hline
\end{tabular}

Figure 87 : Feature Rainure en $U$ 


\subsection{Structure du modèle proposé :}

La structure a été présentée en utilisant le modèle objet normalisé OMT.

Ce modèle de conception par les features, caractérisées par l'association de formes géométriques à d'autres informations relatives à un nombre d'activités du cycle de vie d'un produit mécanique, a la structure suivante :

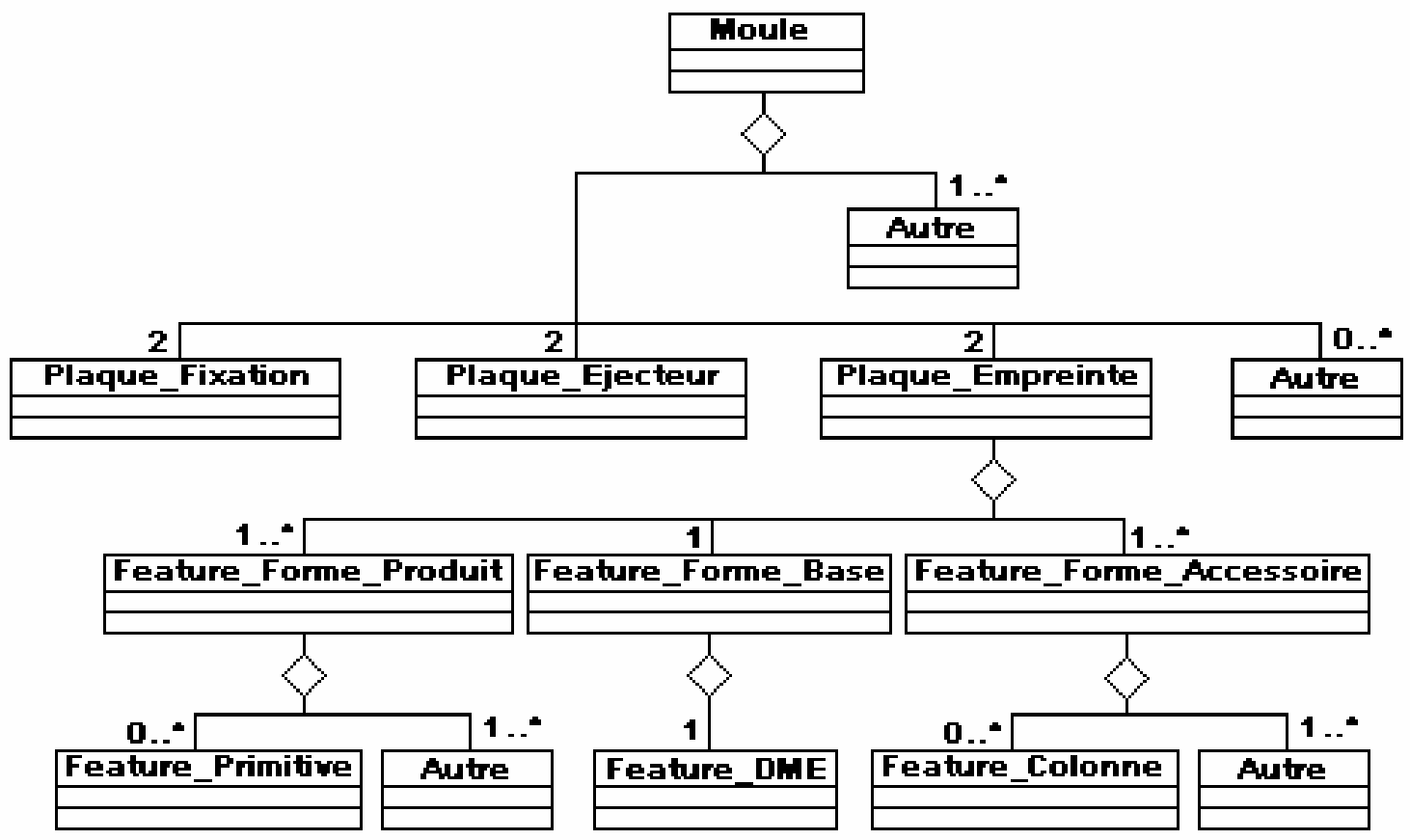

Figure 88 : Modèle OMT du moule d'injection plastique pour le point de vue conception du moule

\subsection{Mise en ouvre informatique :}

La mise en oeuvre informatique a été réalisée pour vérifier la validité du modèle proposé. Pour cela, nous avons opté pour l'environnement de développement orienté objet Visual $\mathrm{C}_{++}$en utilisant les bibliothèques de classes OpenCascade [ $\underline{\mathbf{S 1 0}}$.

La figure 89 représente l'architecture de la maquette baptisée : Mold Feature based Modelling.

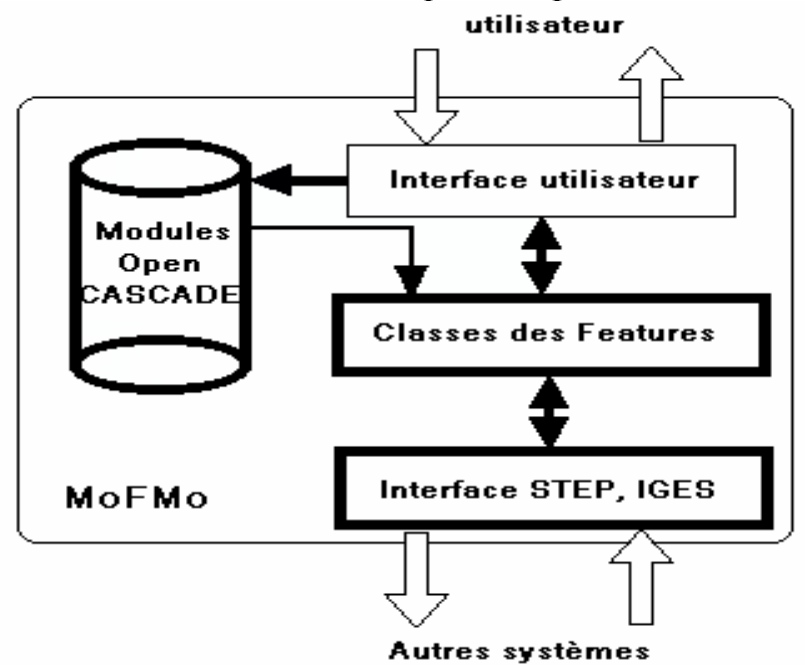

Figure 89 : Architecture de la maquette MoFMo

Les photos d'écran ci-dessous illustre la maquette développé MoFMo: Mold Feature based Modelling. 

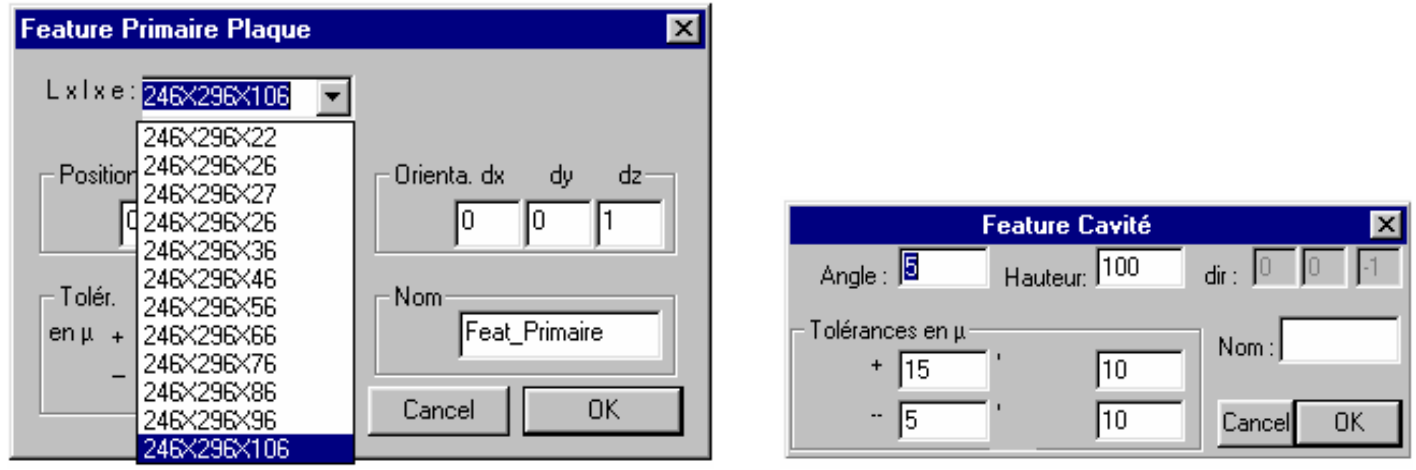

Figure 90 : Insertion d'une feature primaire et d'une feature Cavité dans la feature primaire

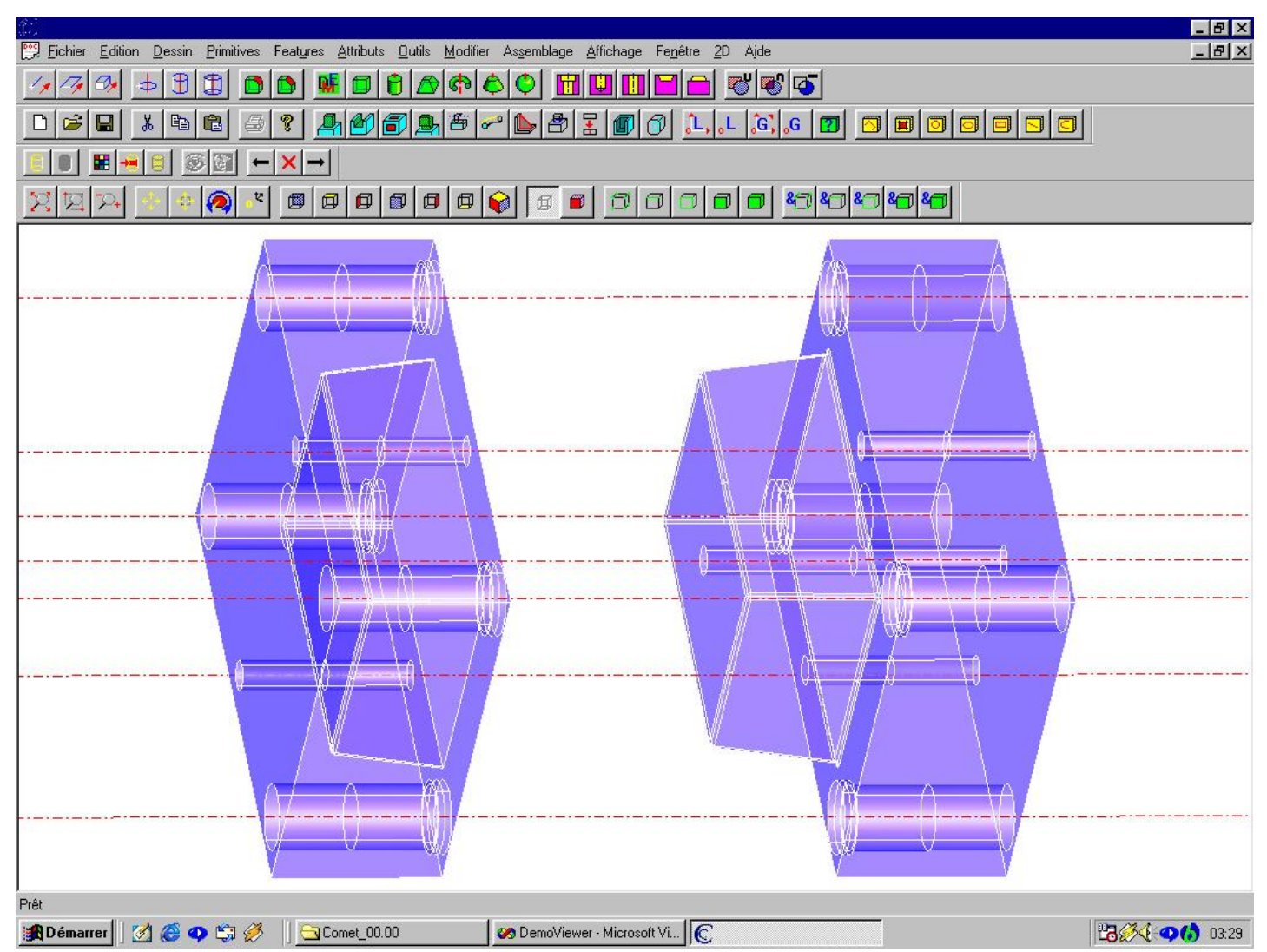

Figure 91 : Copie d'écran de la maquette MoFMo

\subsection{Retombées Scientifiques :}

Ce travail a fait l'objet du Mastère de Mr. F. Bouabda, qui s'est déroulé sous mon encadrement au sein du Laboratoire de Génie Mécanique de l'Ecole Nationale d'Ingénieurs de Monastir. (Voir annexe II, Fiches Encadrement Scientifique : Mastère de Mr. F. Bouabda).

Ce travail a aboutis à une communication nationale (voir Annexe I: Fiches Publication Scientifique) :

- F. Bouabda, A. BenAmara, "Modèle de conception basé sur les entités technologiques: Application à la conception des moules d'injection plastiques“, CRATT' 2002 ISET RADES, Tunisie (2002) 


\section{PARTIE V :}

Conclusions Générales et Perspectives 


\section{PARTIE V}

\section{Conclusions Générales et Perspectives}

\section{Conclusions Générales.}

L'état de l'art présenté dans la partie II de ce mémoire témoigne de façon claire l'impact du problème d'intégration $\mathrm{CAO} / \mathrm{Calcul}$ sur la démarche de conception des produits mécaniques. Les travaux de recherche recensés dans l'état de l'art présenté montrent également que cette thématique est un centre d'intérêt de plusieurs laboratoires de recherche et d'industriels.

Dans ce rapport de synthèse de nos travaux de recherche, nous avons présenté les différentes approches que nous avons élaborées en collaboration avec des collègues chercheurs appartenant à des laboratoires de recherche en Tunisie, en France et au Canada.

Nous avons d'abord commencé par un bref rappel de nos travaux de recherche dans le cadre le la thèse de doctorat dont nous rappelons ci-dessous les principaux résultats :

En effet, en nous basant sur une étude critique des approches présentées en bibliographie, nous avons montré qu'une intégration efficace de la composante calcul dans le processus de conception dépend des trois facteurs suivants :

- Modèle de produit,

- Modèle du processus de conception,

- Modèle d'échange de données.

Nous avons ensuite détaillé l'approche d'intégration CAO/Calcul basée sur les features de calcul qui a fait l'objet d'une thèse de doctorat co-encadré avec des collègues du laboratoire de génie mécanique à l'ENIM et le laboratoire LAMIH de l'Université de Valenciennes en France.

Ce travail a porté sur la formalisation du concept de feature de calcul. Celui-ci est exprimé sous la forme d'un sextuplet, associant trois modèles de données (mécanique, simulation, interprétation) et trois relations de gestion de cohérence entre modèles définis ainsi :

- Le Modèle Mécanique (MM) est une représentation d'un ou plusieurs éléments de la solution de conception établie par le concepteur à partir d'hypothèses liées à la fois à la forme et aux comportements mécaniques de ces éléments, et à la nature des sollicitations (ou conditions aux limites) auxquelles sont soumis ces éléments.

- Le Modèle de Simulation (MS) est un modèle calculatoire du modèle mécanique. Il précise les formules ou plus généralement les procédures de calcul, analytiques ou numériques, susceptibles de produire une évaluation des comportements du système étudié.

- Le Modèle d'Interprétation (MI) est un ensemble de variables d'observation du comportement mécanique du produit que le concepteur souhaite analyser pour évaluer le comportement de la solution retenue ou de ses éléments. Il permet aussi de valider les hypothèses faites au niveau de la construction du modèle mécanique. Ces variables d'observation sont déterminées à partir de la simulation.

- La relation (MM,MS) est une relation de continuité du MM vers le MS, permettant de transférer les données du MM vers le MS. 
- La relation (MS,MI) permet d'extraire de la simulation les variables pertinentes permettant d'analyser, suivant un point de vue déterminé, le comportement de la solution.

- La relation (MI,MM) est une relation de validation, permettant de valider, à l'issue de l'interprétation du comportement mécanique de la solution de conception, les hypothèses de modélisation établies dans le MM.

Dans ce travail les concepts des modèles du domaine technologique et technique (features de calcul cinématique et statique, feature de résistance) ont été développés.

Nous avons ensuite présenté une synthèse sur la deuxième approche d'intégration $\mathrm{CAO} / \mathrm{Calcul}$ qui consiste à développer des outils numériques pour la reconstruction d'un modèle $\mathrm{CAO}$ à partir des résultats de calcul éléments finis.

Ce sujet fait l'objet d'une thèse de doctorat (Borhène Louhichi) que nous co-encadrons avec des collègues du Laboratoire de Génie Mécanique (MA-05) de l'ENI de Monastir (Tunisie) et des collègues du Département de Génie Mécanique de l'Université de Québec à Trois Rivières (Canada).

Ce travail tente d'améliorer le niveau d'intégration entre le domaine de la CAO (modèle géométrique) et le domaine du calcul (calcul éléments finis). Le but est de réinjecter, dans l'environnement de conception $(\mathrm{CAO})$, le modèle $\mathrm{CAO}$ reconstruit à partir des résultats éléments finis (Maillage déformé) afin de donner au concepteur des moyens quantitatifs de validation ou de remise en cause des propositions de conception.

La reconstruction du modèle CAO est basée sur le modèle BREP (Boundary REPresentation) qui a été choisi pour les considérations suivantes :

- Il est unique en le comparant au modèle CSG (Constructive Solid Geometry).

- Il est disponible sur la majorité des modeleurs géométriques.

- Il englobe aussi bien des informations géométriques (la nature des surfaces) que topologiques (les liens entre les différentes entités).

Dans ce travail, trois cas de reconstruction du modèle $\mathrm{CAO}$ à partir des résultats de calcul éléments finis (maillage déformé) ont été considérés :

- Petites perturbations : Ni changement de géométrie, ni changement de topologie.

- Élasticité linéaire : Changement de géométrie sans changement de topologie.

- Elastoplasticité : Changement géométrique et topologique.

Nouas avons montré les résultats obtenus pour les deux premier cas de figure et nous avons soulignés les difficultés rencontrées pour la résolution du troisième volet qui constitue l'une de nos préoccupation de recherche actuelle.

Nous avons ensuite présenté la plateforme de développement orientée intégration CAO/Calcul qui a fait l'objet d'un mastère de génie mécanique en collaboration avec l'UQTR au Canada.

Cette plateforme est constituée d'un ensemble de bibliothèques informatiques (bien structurées et documentées) capables, non seulement de se connecter sur un système de CAO et de générer un maillage optimisé et parfaitement intégré à la $\mathrm{CAO}$ (lien de dépendance entre le modèle CAO et la structure de données maillage et conditions aux limites), mais aussi d'effectuer un calcul éléments finis élastoplastique.

Notons que par rapport à l'utilisation des logiciels standards de calcul par éléments finis (COSMOS, ANSYS, ABAQUS), cette plateforme offre la possibilité d'un accès sans limites à l'ensemble des informations caractéristiques du processus de modélisation : CAO, maillage et calcul. La disponibilité des codes sources de l'ensemble des ressources développées pourra également constituer un avantage par rapport à l'utilisation des systèmes standards clé en main. 
La quatrième partie de ce mémoire a portée sur une synthèse des nos travaux de recherche émergeants qui s'inscrivent dans la thématique « Modélisation et Etude Expérimentale et Numérique des Procédés de Fabrication Mécanique ».

Trois travaux de recherche relevant de cette thématique ont été présentés :

- Simulation numérique du procédé d'emboutissage des tôles minces ;

- Etude expérimentale et numérique du procédé du tournage dur ;

- Modèle de conception des moules d'injection plastique basé sur les entités technologiques.

\section{Perspectives de recherche.}

Nos perspectives de recherche s'articulent autour de deux thématiques présentées dans ce mémoire, à savoir :

- L'intégration $\mathrm{CAO} / \mathrm{Calcul}$

- La Modélisation et Etude Expérimentale et Numérique des Procédés de Fabrication Mécanique

Dans la première thématique nous envisageons de rehausser le niveau d'intégration des outils de $\mathrm{CAO}$ et de Calcul pour répondre aux exigences actuelles d'intégration dans le domaine de la CFAO. Nous estimons que la plateforme de développement orientée intégration $\mathrm{CAO} / \mathrm{Calcul}$ permettra de mettre en œuvre des outils d'intégration capables d'augmenter l'interopérabilité des applications de $\mathrm{CAO}$ et de Calcul.

Nous envisageons également de travailler sur d'autres thématiques en relation avec l'intégration $\mathrm{CAO}$ Calcul, notamment l'enrichissement des modèles de CAO pour prendre en compte les informations requises pour les calculs mécaniques.

Le modèle de produit qui constitue la composante principale du problème d'intégration $\mathrm{CAO} / \mathrm{Calcul}$ doit supporter de façon plus générique les aspects comportement du produit soumis à différentes sollicitations et ce à différents niveaux de modélisation. Ces entités (features de comportement) doivent supporter l'ensemble des informations nécessaires pour dimensionner, optimiser et évaluer le produit aux différents niveaux de la conception. Nous envisageons également d'intégrer dans le modèle de produit d'autres caractéristiques permettant la prise en compte des aspects coût et fiabilité. Ces caractéristiques sont importantes pour le choix de la technologie de réalisation des différents composants du produit et le choix du type de calcul de comportement à effectuer dans une phase de modélisation donnée.

L'aspect modèle de processus qui joue un rôle important pour supporter l'intégration fera aussi partie de nos perspectives de recherche. Notre objectif est de mettre au point des modèles permettant de supporter de façon systématique le déroulement opportun du processus de conception, sans imposer au concepteur un ordre figé d'exécution des tâches de conception. Le concepteur doit avoir la possibilité de naviguer dans la structure des données en fonction de la nature du problème de conception à résoudre (processus non monotone). En effet, le concepteur peut partir d'une spécification fonctionnelle du produit pour aboutir à une description technique de ces différents composants (approche fonctionnelle), et/ou il peut utiliser certains composants préexistants pour concevoir de nouveaux produits (re-ingéniering).

En ce qui concerne les outils numériques de retour du calcul vers la $\mathrm{CAO}$, nous envisageons de résoudre le problème de reconstruction avec changement de géométrie et de topologie et de valider les approches proposées sur des exemples industriels.

Ce retour est actuellement envisagé avec un point de vue statique. Nous projetons également de travailler sur la reconstruction en temps réelle (assemblage en état de fonctionnement) qui nécessite des algorithmes de calcul rapides (calcul parallèle). 
Dans la deuxième thématique, nous envisageons de poursuivre nos travaux sur l'expérimentation et la simulation numérique des procédés de fabrication mécanique notamment en ce qui concerne la modélisation de la coupe qui constitue un axe d'actualité vu les évolutions des techniques de la coupe des métaux. En effet, Les progrès remarquables des machines outils à commande numérique (rigidité, puissance, précision des déplacements et de commande et plages des vitesses.) couplées avec le développement des outils de coupe (formes, matériaux et revêtement) ont permis d'augmenter le champs d'application de ces procédés, comme l'usinage des aciers durcis et l'UGV, et d'améliorer la précision dimensionnelle et la qualité des surfaces usinées.

Parmi nos objectifs dans ce domaine, on se propose dans le cadre d'une thèse en cours, d'élaborer des modèles de prédiction de la qualité des surfaces usinées en fonction des paramètres de coupe en usinage 3axes avec une fraise à bout sphérique. Ce modèle de prédiction, basé sur des réseaux de neurones, sera utilisé pour optimiser le choix des conditions de coupe (vitesse, avance, profondeur de passe, type de trajectoire, etc.) en vue d'améliorer la qualité de la surface usinée. L'apprentissage des réseaux de neurones sera basé sur une compagne d'essaies expérimentaux organisée selon des plans d'expérience appropriés. 
BIBLIOGRAPHIE : 


\title{
BIBLIOGRAPHIE
}

\begin{abstract}
[Anderson 90]: $\quad$ D.C.Anderson, T.C.Chang; < Automated process planning using object-oriented feature based design, in Advanced Geometric Modelling for Engineering Applications>; eds. Krause F.-L., Jansen H., Elsevier, IFIP/GI, 1990.

[Arabshabi 91] S. Arabshabi, D. C. Barton, N. K. Shaw, "Towards integrated design and analysis”, Finite Element in analysis and design, vol 9, pp 271-293, Elseiver Science, 1991.

[Armstrong 96] C. G. Armstrong, R. J. Donaghy, and S. J. Bridgett, "Derivation of appropriate Idealisation in Finite Element Modeling", the 3ème international conference of computational structures technology, Budapest 1996.
\end{abstract}

[Ashaab 94] Al-Ashaab, A.H, "A Manufacturing Model To Capture Injection Moulding Process Capabilities To Support Design For Manufacture", Thèse de doctorat de l'Université de Loughborough, 1994.

[Banningen 95] C.J.H.V. Banningen, Feature modeling, Report 1, Delft University of Technology, Faculty of Industrial Design Engineering Department of ～Engineering Design Kmeno 1995.

[Belaziz 99] M. Belåǐ "Simplification et idéalisation de modèles de formes caractéristiques dans un contexte d'intégration CAO/Calcul», Université Lyon 1, 1999.

[BelHadjSalab 03] Hedi Bel Hedj Salah ; "Rapport d’habilitation » Laboratoire de Génie mécanique 2003.

[Benamara 987 A. Benamara, "Contribution à l'intégration de la composante calcul dans une démarche de conception fonctionnelle intégrée, application aux mécanismes ", mémoire de doctorat, université de Valenciennes, décembre 1998.

BenAmara 007 A. Benamara, N. Aifaoui, D. Deneux, Intégration CAO/Calcul, une démarche fonctionnelle intégrée, JESA, Vol 34, n²-3, pp 247-258, Avril 2000.

[Bennis 00] F. Bennis, P. Chedmail, O. Helary, "Representation of design activities using neural networks: application to fursy dimensioning, IDMME2000.

Booch 00] G. Booch, J. Rumbaugh, I. Jacobson, Le guide l'utilisateur UML, Eyrolles Paris, 2000.

[BOULILA 00] A. BOULILA; «Etude expérimentale et modélisation numérique du gonflement bydraulique des plaques minces»; Rapport de DEA, ESSTT, 2000

[Breitfield 96] T. Breitfield, B. Kroplin, "An expert system for the verification of finite-element calculations", Actes de SAST'96, IEEE, 1996.

[CHABENAT 03] A. CHABENAT et R. MARTIN; «Découpage et Poinconnage ; Technique de l'ingénieur, B7 561, ph. 1-22, 1995.

[Cuillère $937 \quad$ J. C. Cuillère, "Pré-optimisation de maillages automatiques tridimensionnels pour les méthodes numériques, application à l'ingénierie simultanée ", Thèse de doctorat, Université de Lorraine, 1993.

[Cuillère 99] J. C. Cuillère, R. Maranzana, "Automatic and a priori refinement of three dimensional meshes based feature recognition techniques", Advanced in engineering software, 1999. 
[Dabke 94] P. Dabke, V. Prabhakar, S. Shepard, "Using features to support Finite Element idealization", International conference ASME, Minneapolis, vol 1, pp 183-193, 1994.

[Dangy 94]: F.Dangy, F.Féru; <FEMOD: A Feature Based Modelleur Architecture>; Advanced CAD/CAD Systems; IFIP, V alenciennes, France 1994.

[Degirmenciyan 95]: I.Degirmenciyan; <Du Paradigme Essai-Erreur> au Paradigme du Choix Dirigé, Application à la Conception Mécanique, Acteur Unique, Acteurs multiples>; Thèse, Ecole Centrale de Paris 1995.

Erve 887:

A.H.V.Erve; <Computer Aided Process Planning for Part Manufacturing, an expert system approach>, PhD de l'Université de Twente, 1988.

Falco 01$]$

G. Falco, F. Noël, "Spécification d'un outil pour la gestion des processus d'analyse mécanique", 7ème Colloque sur la conception mécanique intégrée, AIP-PRIMECA, La plagne, pp 26-33, 2-4 avril 2001.

[Faltings 92] B. Faltings, "A symbolic approach to qualitative kinematics", Artificial intelligence Journal, 56 pp 139-170, 1992.

[FEAST 94]: $\quad$ FEAST; <Feature Based Assembly Modelling >; Project Fonded By the European Community Under the BRITE/EURAM Program : No BRE2-CT94-1015; Aerospatiale, Alenia, Dassault Aviation, Casa, Université de Parme, Université de Valenciennes; 1994-97;

[Femex 96]: $\quad$ FEMEX: An International Working Group for FEature Modeling EXperts; <Feature Definition" ; http://www.igd.fhg.de/www/ qgdv/AKs/; Mars 1996.

[Feng 01] C. Feng, "An experimental stady of the Impact of Turning Parameters on Surface Roughness", Industrial Engeneering Conférences, 2001, Paper $N^{\circ} 2036$.

[Feng 02] X. Feng, X. Wang, "Development of Empirical Models for Surface Roughness Prediction in Finish Turning", International Journal of Advanced Manufacturing Technology, Vol. 20, 2002, pp. 348-356.

[Fenves 86] S. J. Fenves, "A framework for cooperative development of finite element modelling assistant ", Actes de conference on reliability of methods for engineering analysis swansea 1986.

[Ferraille 98] F. Ferraille, "regroupement des points de vue en conception intégrée ", Thèse de doctorat, Ecole centrale de Nantes, 1998.

[FERU-94] Féru F., Vat Ch., Cocquebert E., Deneux D., Rouchon C. "Computer-aided functional designfor-manufacturing". Revue Internationale de CFAO et d'Infographie - Vol 9, N5, 1994.

[Fine 01$] \quad$ L. Fine, J. C. Léon, "Multi-représentation et intégration de modèles dans le processus d'analyse mécanique", 7ème Colloque sur la conception mécanique intégrée, AIP-PRIMECA, La plagne, pp 66-73, 2-4 avril 2001.

[Fischer 00] X. Fischer, "Stratégie de conduite du calcul pour l'aide à la décision en conception mécanique intégrée, application aux appareils à pression ", Thèse de doctorat, Ecole Nationale d'Arts et Métiers, Centre de Bordeaux, 2000.

[Fjellheim 83] R. Fjellheim, „An expert system for SESAM 69'Report C. P. 83-6010, Dpt of telematicscomputers, Det Norske Veritas, Norway, 1983.

[François 98] V. François, "Méthodes de maillage et de remaillage automatiques appliquées à la modification de modèle dans le contexte d'ingénierie simultanée », Thèse de doctorat, Université Henri Poincaré, Nancy 1, 1998. 
[Francois 98] V. Francois, Automatic meshing and remeshing methods applied to model modification in the simultaneous engineering context (paper in French), PhD. Thesis. University Henri POINCARE, Nancy I (France). November 1998.

[GAIECH 01$] \quad$ Z. GAIECH ; «Simulation numérique du gonflement bydraulique des tôles minces»; Rapport de DEA, UTC-UTT, 2001

[Geffrier 91]: C. Geffrier; <Une filière complète de conception et de calcul de structures dans CATLA>; Dassault système; actes de MICAD 1991.

[Ghias 01]

K. Ghias, A. Mohamed, M. Lemaire, "Une nouvelle méthodologie pour la conception optimisée des structures fiables”, 7ème Colloque sur la conception mécanique intégrée, AIP-PRIMECA, La plagne, pp 26-32, 2-4 avril 2001.

[Ghodous 96]: P.Ghodous, D.Vandorpe; <Modélisation Intégrée de Données de Produit et de Processus $>$; Revue internationale de CFAO et d'informatique graphique, Hermès 1997.

[Giacometti 91]: F.Giacometti, T.Chang; <Design of mechanical assemblies with functional features>; Design Automation Conference Miami Sept. 1991.

[Gossard 887: $\quad$ D.C.Gossard, R.P.Zafarite, H.Sakurai; <Representing dimensions, tolerances and features in MCA systems $>$; IEEE march 1988.

[Helary 00] O. Helary, "Conception intégrée: pré dimensionnement en environnement imprécis", Thèse de doctorat, Université de Nantes, 2000

[Hyang 04] Y. Hyang, S. Y. Liang, "Modeling of CBN tool flank wear progression in finish hard turning", ASME J. Manuf. Sci. Eng. 126 (1) 2004.

[Joskowicz 95] L. Joskowicz, E. Sacks, "Computational kinematics" ASME, Journal of mechanical design, $117,1995$.

[Jung 00]

[Kruth 98 ]

[Kurowski 95$]$

H. B. Jung and K. Kim; "A New Parameterisation Method for NURBS Surface Interpolation”, The International journal of Advanced Manufacturing Technology, pages 784-790, 2000.

J. - P. Kruth, A. Kerstens, Reverse engineering modelling of free-form surfaces from point clouds subject to boundary conditions. Journal of Materials Processing Technology 76 (1998) 120-127.

M. P. Krurowski, „When good engineers deliver bad FEA“, Machine Design Edited by Paul Dvorak, November, 1995.

[Laako 90] : $\quad$ T.Laakeko, M.Mentyle, R.Mentyle, J.Nieminen, R.Sulonen, J.Tuomi; <Feature models for design and manufacturing >; 23 rd Hawai International Conference on System Sciences, IEEE, Vol.II ed. Shriver B.D., 1990.

[Labat 92]: A. Labat, E. Le Bègue, M. Castellanet; <Le lien CAO/Analyse à Aérospatiale Cannes, La Solution PARALEL"; Esprit Concept \& Aerospatiale Cannes; MICAD 1992.

[Lagache 84] J. C. Lagache, „Présentation du système expert pour le code TITUS ", Framentec, 74Bd d'Italie, Monaco, 1984

[Lalane 87] B. Lalanne, Système expert d'aide à l'analyse du comportement mécanique des systèmes, Thèse de doctorat, Université de V alenciennes, 1987.

[Lalanne 87] B. Lalanne, „Système expert d'aide à l'analyse du comportement mécanique des systèmes“, Thèse de doctorat, Université de V alenciennes, 1987. 
[Lee 96] R.J.V. Lee, An Injection Moulding Strategist In Information Modelling Environment, thèse de doctorat de l'université de Loughborough 1996

[Lee 97] Lee R.S., Lee C.Z., Chen, Y., "Development of a concurrent mould design system: A knowledge based approach." Computer Integrate Manufacturing Systems, 1997

[Loriod 89] Georges Loriod, Contribution à une approche CAO produit, proposition d'un modeleur géométrique", Thèse de doctorat de l'Université de Valencienne 1996

[Loriod 89] G.Loriod; <Contribution à une Approche CAO Produit, Proposition d'un Modeleur Géométrique>; Thèse de l'Université de Valenciennes 1989.

[Lubye Dixon 86] : S.C.Luby, J.R.Dixon, M.K.Simmons; <Designing with features: creating and using a features database for evaluation of manufacturability of castings $>$; ASME Computers in Engineering Conference 1986.

[Mantyla 98]

M. Mantyla, An introduction to solid modeling, Computer science press, 1998.

[Marcal 80]

P. V. Marcal, M? B. Hsu, J.M. Rubin, "Knowledge based consultation for finite element structural analysis", TR-80-6039, Marc analysis research corporation, 1980.

[Marty 91]: C.Marty; <Le Mieux produire : approche productique du management>; Paris Lavoisier - Tec \& Doc 1991.

[Mawussi 95a]: K. Mawussi, A. Bernard; <Représentation et Aide à la Définition d'Outillage de Forme Complexe Basée sur un Modèle d'entités Complexes>; Revue internationale de CFAO et d'informatique graphique, Vol. 10, n5, 1995.

[Mobley 96$]$ A. V. Mobley, M. P. Carroll, S. A. Canann, "An object oriented approach to geometry defeaturing for Finite Element Meshing, Proceedings of 7ème international roundtable, Sandia National Laboratories, 1998.

[Montogomery 97] D. C. Montogomery, "Introduction to statistical quality control", John Wiley \& Sons, Inc. 3rd Edition 1997.

[Morris 97] A. J. Morris, R. Vignjevic, "Consistent finite element structural analysis and error control", Computer methods in applied mechanics and engineering, Elseiver Science, vol 140, pp 87-108, 1997.

[Narayanin 92] : $\quad$ M.Narayanin; <La Liaison CAO-Calcul>; Matra DataVision; actes de MICAD92

[Özel 03$] \quad$ T. Özel; «Modeling of hard part machining: effect of insert edge geometry in CBN tools regression»; IIE Transactions, Vol. 35, 2003,

[Papegay 92]: $\quad$ Y.Papegay; <Outils Formels pour la Modélisation en Mécanique>; Thèse de l'Université de Nice Sophia-Antipolis, Ecole Doctorale des Sciences pour l'Ingénieur; Nice, France 1992.

[Petian 95]: C.Petiau; <Calcul et conception des structures>; DASSAULT AVLATION; actes de MICAD 1995.

[Piegl 01] L.A Piegl, W. Tiller, Parametrization for surface fitting in reverse engineering. Computer-Aided Design 33 (2001) 593-603.

[Piegl 97] L.A. Piegl and W. Tiller. The NURBS book. Springer-Verlag, 1997.

Poulachon 007 G. Poulachon, A. Moison, "Hard turning Cutting Mechanisms and Metallurgical Aspects". Trans. ASME J.of Eng. For Ind. Mai 2000. 
[Poulachon 99] G. Poulachon, "Aspects phenoménologiques et métallurgiques en tournage c-BN des aciers durcis. Application : usinabilité de l'acier 100Cr6". Thèse de doctorat de l'ENS AM de Cluny 1999.

[Pratt 93] M. J. Pratt, S. Meeran, "Automated feature recognition form 2D drawings", Computer Aided Design, Volume 25, num. 1, 1993, pp 7-17.

[Pratt 93]

[Remondini 94]

Pratt M. J., "Application of feature recognition in the product life cycle", International Journal of Computer Integrating Manufacturing, 1993.

L. Rémondini, J. C. Léon, A. Trempette, M. Kutniak, L’approche par entités dans la modélisation des structures mécaniques, Actes des conférences internationales IFIP, Modélisation et reconnaissance des caractéristiques dans les systèmes de CFAO avancés, Valenciennes, Mai 1994, pp 432-447.

[Ren 02]

[Requicha 86]:

Composite freeform surface reconstruction using recursive interpolating subdivision scheme. B.Y. Ren, I. Hagiwara Computers in Industry 502003.

A.A.G.Requicha, S.C.Chan; <Representation of Geometric features, tolerances and attributes in Solid Modeleurs based on Constructive Géometry >; Journal of Robotics \& Automation IEEE, Vol RA 2, n³, September 1986.

[Rezayat 96] M. Rezayat, "Midsurface abstraction from $3 \mathrm{D}$ solid models: general theory and application", computer aided design, vol 28, pp 905-915, 1996.

[Robert91] G. Robert, R. Ficher, "Optimisation structurale avec STRIM100", Revue de CFAO et d'Infographie, vol 5, num 4, pp 73-102, 1991.

[Roller 89]: $\quad$ D.Roller, <Design by Features : An Approach to High Level Shape Manipulations>; Hewlett Packard GmbH Germany; Computers in Industry, Elsevier Science Publishers B.V 1989.

[Saguez 95]: C. Saguez; D. Begis; F. Eichelbrenner; <Intégration CAO/Calcul>; Simulog-France; actes de MICAD 1995.

[Salomons 93]: O.W.Salomons, F.J.A.M.Van Houten, H.J.J.Kals. <Review of research in feature-based design>; Journal of Manufacturing Systems, Vol. 12, No.2, 1993.

[Salomons 95a]: O.W.Salomons; <Computer Support in the Design of Mechanical Products: Constraint specification and satisfaction in feature based design for manufacturing $>$ Thèse de Doctorat de l'université de Twente, Enschede 1995.

[Salomons 95b]: O.W.Salomons, J.Poerink, H.J., Slooten F. van Houten F.J.A.M. van Kals H.J.J.; <A Computer Aided Tolerancing Tool Based on Kinematic Analogies>; Les annales du CIRP, Seminaire sur <Computer Aided Tolerancing>, The University of Tokyo, JAPAN, April 5 $6,1995$.

[Sam 95] J. Sam, Constraint consistency techniques for continuous domains", Thèse de doctorat, Ecole polytechnique fédérale de Lausanne, 1995.

[Shah 91] J.J. Shah and A. Mathew, "Experimental investigation of the STEP form-feature information model", Computer Aided Design, Volume 23, num. 4, 1991, pp 282-296.

[Shab 94] K. P. Shah, S. A. Meguid, A. Zaugas, "Development of software for integrating dynamic analysis for multi-body systems", Computer Aided Design, vol 26, num 2, pp 109-118.

[Shah 88]: J.Shah, M.Rogers; <Functional Requirements and Conceptual Design of the Feature-Based modelling System >; Computer Aided Engineering Journal, February 1988. 
[Shah 89]:

Shah 90a]:

Shah 90b7:

[Shah 90c]:

SShah 96]:

[Shah 96$]$

[Sheffer 97]

[Shepard 90]

Stab 917:

[Steven 01]

[Steven 02$]$

[Szabo 96]

[Tehari 99]

[Troussier 99]

[Vignjevic 98]

[Volpin 98]
J.Shah, M.Rogers, P.Sreevalson, A.Mattehew; <Functional Requirements for Feature-Based Modeling>; Report R-89-GM01, CAM-I, July 1989.

J.Shah \& al; <The ASU Features Testbed>; Technical Report, Mechanical and Aerospace Engineering Departement, Arizona State University 1990.

J.Shah; <Philosophical Developpement of Form Features Concept>; CAM-I, Features Symposium, Boston August 1990.

J.Shah, M.Rogers, P.Sreevalsan, D.Hasio, A.Mathew, A.Bhatanagar, B.Liou, D.Miller; $<A n$ OverView of the ASU Features Testbed $>$, Computers in Engineering Conference, ASME, Boston August 1990.

J.Shah, S.Y.Yan; <Representation and Mapping of Geometric Dimensions from Design to Manufacture>; Design Engineerin Technical Conference and Computers in Engineering Conference, ASME Irvine Californie 1996.

J. Shah, M. Roger, P. Sreevalsan, D; Hasio, A. Mathew, A. Bhatanagar, B. Liou, D. Miller, An overview of the ASU Features tested, Computer in Engineering Conference, ASME Irvine California 1996.

A. Sheffer, T. D. Blacker, M. Clustering, "Automated detail suppression using virtual topology", ASME, pp 57-64, 1997.

M. S. Shepard, E. V. Korngold, R. Wentorf, "Design systems supporting engineering idealizations", actes de IFIP 90, Geometric Modeling for Product Engineering, M. J. Wozny, J. U. Turner and K. Press Editors, Elseiver Science Publishers B. V., North Holland, 1990.

O.Stab, J.M.Drappier; <Opérations booléennes sur les maillages, application au maillage automatique >; ENSMP; actes de MICAD 1991.

Steven J. Owen and David R. White, Mesh based geometry: A systematic approach to constructing geometry from a finite element mesh, 10th International Meshing Roundtable Newport Beach, California, U.S.A. October 7-10, 2001.

Steven J. Owen, David R. White, Timothy J. Tautges, Facet-based surfaces for 3D mesh generation 11th international meshing roundtable, September 15-18, 2002, Ithaca, New York.

B. A. Szabo, "The problem of model selection in numerical simulation", Advances in computational methods for simulation, B. H. V. Topping Editor, Civil-Comp Press, Edinburgh, pp 9-16, 1996.

A. Tehari, «Analyse morphologique de modèles pour décrire un produit par des caractéristiques en fonction du point de vue », Thèse de doctorat, Université Lyon 1, 1999.

N. Troussier, "Contribution à l'intégration du calcul mécanique dans la conception des produits techniques, proposition méthodologique pour l'utilisation et la réutilisation ", Thèse de doctorat, Université Josef Fourier, Grenoble 1, 1999.

R. Vignjevic, A. J. Morris, A. D. Belagundu, "Towards high fidelity finite analysis », Advances in engineering software, Elseiver, science, Civil-Comp Press, Edinburgh, vol. 9, $n^{\circ} 79$, pp 655$665,1998$.

O Volpin, A Sheffer, $M$ Bercovier and Joskowicz, Mesh simplification with smooth surface reconstruction. Computer aided Design, Vol 30, n¹1, pp 875 - 882, 1998. 
[Walton 96] DJWalton and DS Meek, A triangular G1 patch from boundary curves. Computer Aided Design, Vol. 28, pp 113-123, 1996.

[Zhongwei 04] Zhongwei Yin, Reverse engineering of a NURBS surface from digitized points subject to boundary conditions. Computer \& Graphics 28 (2004) 207-212.

\section{Sites Internet :}

wnw.salome-platform.org/

bttp:// wmw.msh-alpes.prd.fr/IPI/Projets IPI/2001-2003/projet/projets finances par l IPI.btml

bttp:/ gilco.inpg.fr./

http://gilco.inpg.fr/projets/Oscar/

bttp:/ / wnw.dragon.uni-karlsrube.de/

bttp:// eu-datagrid.web.cern.ch/eu-datagrid/

bttp:/ / wmw.3s.hmg.inpg.fr/

http:/ / wmw.lag.ensieg.inpg.fr/

bttp:/ / www-lmc.imag.fr/

bttp:// mmw.opencascade.com/ 


\section{ANNEXE I :}

Fiches Publication Scientifique 
Fiches Articles de Revue

$$
\text { (1996- 2005) }
$$




\title{
FICHE PUBLICATION SCIENTIFIQUE
}

\section{ARTICLE DE REVUE}

Titre : « Intégration $\mathrm{CAO} / \mathrm{Calcul}$ par reconstruction des modèles $\mathrm{CAO}$ à partir des résultats de calcul.»

Auteurs : $\quad$ Borhen Louhichi*, Abdelmajid Benamara*, Vincent François**

\author{
Laboratoires Impliqués : \\ * Laboratoire de Génie Mécanique (Lab. Ma-05). \\ ** Département de Génie Mécanique, Université du Québec à Trois Rivières.
}

Nom de Revue / Congrès : $\quad$ REVUE INTERNATIONALE D'INGENIERIE NUMERIQUE, VOLUME $1-\mathrm{N}^{\circ} 1 / 2005$.

Etat actuel : $\quad$ Publié Sept 2005.

\section{Résumé détaillé :}

Dans un contexte d'ingénierie simultanée, l'intégration entre les différentes étapes de modélisation devient de plus en plus une nécessité. En effet, les problèmes d'intégration s'avèrent les premiers responsables de perte de temps durant la phase d'optimisation.

Le travail présenté dans ce papier s'inscrit dans le cadre de l'intégration CAO FAO et Calcul, nous commençons par introduire l'intégration et son utilité. Par la suite l'état de l'art et nos travaux au cours de temps sont présentés tout en montrons leurs apports dans l'intégration. Dans une dernière partie nous proposons une démarche de reconstruction des modèles $\mathrm{CAO}$ à partir des résultats d'une analyse éléments finis dans le cas élastique. La démarche consiste à reconstruire un modèle de type BREP à partir des résultats de calcul qui est le maillage déformé. L'algorithme général se base sur une recherche de la topologie et une reconstruction des entités géométriques. L'algorithme se défère suivant le type des déformations subites par le système. Dans notre démarche, on caractérise les cas de reconstruction suivant les changements topologiques et géométriques sur le modèle. Trois cas sont possibles à savoir :

- Cas des petites perturbations : Dans ce cas la topologie ne change pas et la nature des faces et arêtes reste la même. Mais les caractéristiques des différentes entités géométriques peuvent changer (vecteur directeur, point d'insertion...).

- Cas des changement géométriques: Dans ce cas les déformations engendrent sur les différents entités de modèle des changements géométriques mais la topologie reste la même.

- Cas des changements topologiques et géométriques: C'est le cas extrême de déformations (élasto-plasticité). Une recherche de la topologie, dans ce cas, avant la reconstruction de la géométrie est nécessaire.

Le développement du premier cas nous permettra ensuite de montrer, à la base d'un exemple, l'efficacité de la méthode proposée et son intérêt. Actuellement dans tous les systèmes de CAO, la simulation cinématique d'un assemblage est réalisée avec des corps rigides non contraints. Cette hypothèse est satisfaisante dans la plupart des cas. Cependant dans quelques cas, il serait souhaitable d'effectuer la simulation en tenant compte de l'état contraint des pièces (états déformés des pièces). Dans la simulation nous illustrons également les interférences et les collisions entre les différentes composantes du système dues aux déformations. En fin, les conclusions et les perspectives sont présentées. 


\section{FICHE PUBLICATION SCIENTIFIQUE}

\section{ARTICLE DE REVUE}

Titre : " Identification of the hardening curve using a finite element simulation of the bulge test.»

Auteurs : $\quad$ Abdessalem Chamekh*; Amen GAhbiche*; Hedi Belhadj Salah*; Abdelmajid BenAmara*; Abdelwaheb Dogui*

Laboratoires Impliqués : * * Laboratoire de Génie Mécanique (Lab. Ma-05).

Nom de Revue / Congrès : $\quad$ Steel GRIPS Journal of steel and related materials; Septembre $2004741-746(2004)$

Etat actuel : Publiée Sept 2005.

\section{Detailed Abstract:}

Nowadays, the forming process of sheet metals is a significant industrial activity. In order to control this process, the numerical simulation seems to be the best tool. In fact, with the recent developments in the non-linear computational tools, we can make tests, compare and optimize these processes at a low cost. In fact, the numerical simulations are actually used to control and optimize the forming processes. The quality of these simulations strongly depends on the efficiency of the behaviour models of the deformed materials. In order to identify such models, experimental tests must be used. In this work, the bulge test with circular and elliptical dies are considered. During the bulge test, the displacement of the central point and the pressure were continuously monitored and recorded. The numerical simulations of this test are carried out using ABAQUS software. These current simulations, the behaviour of the specimen are assumed to be elastoplastic.

The Hill's criterion (48) and the isotropic hardening are considered. An inverse modelling based on the finite element computations and experimental data is made in several situations. The aim of this analysis is to identify the parameters of the hardening curve and the Lankford's coefficients. Several sets of results are presented and discussed.

The experimental test concerns a thin steel disk, clamped along its boundary by a draw-bead between the blank-holder and the die and subjected to a uniform pressure $\mathrm{P}$ on one face (Fig. 1). e0, e and $\mathrm{h}$ represent, respectively, the initial thickness, the final thickness and the height of the central point. One of the significant advantages of this test is in the fact that it held almost without friction. The geometrical data and a sketch of the experimental dies are shown in Fig. 2 for the circular die and the elliptical die (Fig 3). The steel disk has a radius of $66.5 \mathrm{~mm}$ and a thickness of $0.8 \mathrm{~mm}$. The part exceeding the snap ring is neglected. The circular die is a revolution shell having an external radius of $66.5 \mathrm{~mm}$ and an internal radius of $45.5 \mathrm{~mm}$. The elliptical die has a $110 \mathrm{~mm}$ along the large axis and a $74 \mathrm{~mm}$ along the small axis. The two dies have a $24 \mathrm{~mm}$ of height and a radius of $6 \mathrm{~mm}$ for the bearing section. During the bulge test, the displacement of the central point Up and the pressure $\mathrm{P}$ were continuously monitored and recorded.
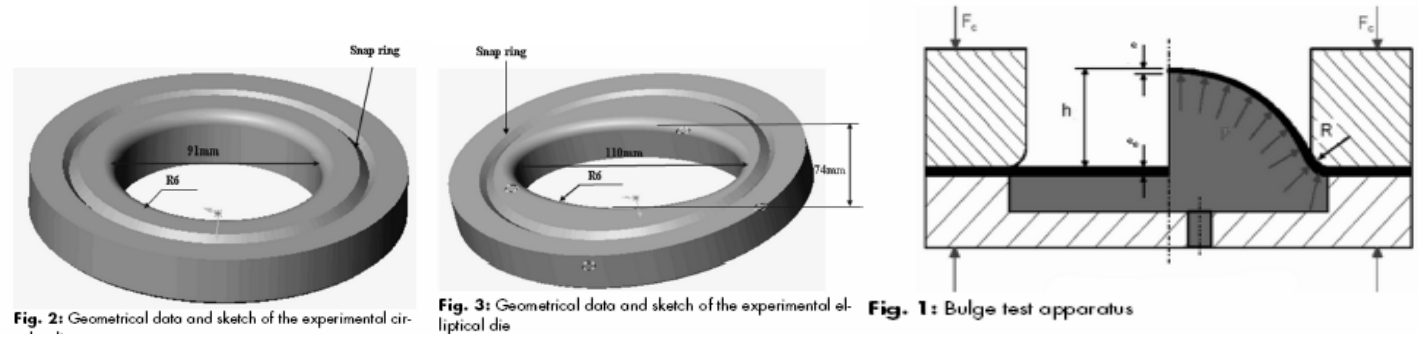


\section{FICHE PUBLICATION SCIENTIFIQUE}

\section{ARTiCle DE REVUe}

Titre : " Interopérabilité des processus conception et calcul. Une approche basée sur les features "

Auteurs : $\quad$ N. Aifaoui, A. BenAmara, A. Dogui

Laboratoires Impliqués : * Laboratoire de Génie Mécanique (Lab. Ma-05).

Nom de Revue / Congrès : Journal Européen des Systèmes Automatisés, volume, 38, numéro 3-4 2004.

Etat actuel : $\quad$ Publié en décembre 2004

\section{Résumé détaillé :}

De nos jours, l'activité de conception de produits industriels est soumise à des contraintes sévères de coût, de qualité et de délais. Pour mieux répondre à ces contraintes, l'intégration efficace des différentes tâches de conception et de calcul est nécessaire.

Dans cet article une approche originale permettant un cadre général d'interopérabilité entre les processus parallèles de conception et de calcul a été introduite. Cette approche est basée sur le concept de feature de calcul. Ce concept a été modélisé par un sextuplet ( 3 modèles de données, 3 relations de cohérence) globalement adapté à la caractérisation et à la résolution des problèmes de calcul typiques identifiés à partir d'une analyse de plusieurs cas de conception. Un catalogue de features a permis de structurer un nombre restreint de features de calcul particulières. Pour avoir une représentation normalisée, le formalisme UML a été utilisé pour décrire le modèle de données du catalogue ainsi que les processus de choix et d'instanciation de features à partir du catalogue. La validation de la méthode a porté sur la conception d'un système de fermeture d'une machine à mouler.

L'approche proposée permet de supporter différents problèmes de calcul typiques couramment rencontrés dans la conception des systèmes mécaniques. Elle assure la traçabilité du calcul en vue d'une réutilisation afin de réduire le temps de sa mise en œuvre dans la conception. Cependant, pour être réutilisée dans un système de CAO intégré, un travail de codification et d'indexation des traces est nécessaire. D'autre part, le concepteur devient plus réactif dans la mesure où il peut intervenir dans tous les niveaux de modélisation du calcul en vue d'apporter des modifications ou de faire du recalcul utilisant d'autres variables d'observation caractérisant d'autres points de vue de l'analyse mécanique. 


\section{FICHE PUBLICATION SCIENTIFIQUE}

\section{ARTiCle DE REVUe}

Titre : "Intégration CAO/Calcul, une démarche fonctionnelle intégrée "

Auteurs : $\quad$ A. Benamara*, N. Aifaoui*, D. Deneux **

Laboratoires Impliqués : * Laboratoire de Génie Mécanique (Lab. Ma-05).

Nom de Revue / Congrès : Journal Européen des Systèmes Automatisés, volume, 34, numéro 2-3 2000.

Etat actuel : $\quad$ Publié en avril 2000

\section{Résumé détaillé :}

Nous avons présenté dans ce papier une approche originale de conception fonctionnelle intégrant la composante calcul dans le processus de conception. Nous avons commencé par exposé la nécessité de l'intégration dans la conception des produits, et cela pour répondre aux exigences des nouvelles approches de l'ingénierie de conception.

La deuxième partie de ce papier a portée sur la définition des concepts de l'intégration et plus particulièrement l'intégration $\mathrm{CAO} / \mathrm{Calcul}$. Cela nous a permit d'identifier les facteurs clés de l'intégration $\mathrm{CAO} / \mathrm{Calcul}$. Nous avons ensuite détaillé le modèle de produit proposé en se basant sur le formalisme de représentation orientée objet OMT.

Dans la dernière partie de ce papier nous avons présenté la mise en œuvre informatique des modèles proposés Cela nous a permis de mettre en évidence les principales fonctionnalités de ces modèles :

- Son aptitude à supporter les informations requises au calcul mécanique

- Son aptitude à gérer la propagation des contraintes géométriques et technologiques engendrées par les tâches de calcul et d'évaluation ;

- Son aptitude à échanger les informations avec les outils d'analyse en conservant la sémantique associée.

Nos perspectives de recherche s'orientent actuellement vers une intégration multi-métiers. Pour cela, nous envisageons de développer les aspects, modèle de produit et modèle de processus, afin de pouvoir, d'une part, supporter l'ensemble des informations requises à une telle intégration et d'autre part, pour donner plus de flexibilité au concepteur lors de la conception. Nous comptons également développer l'aspect échange de données entre les différents outils de modélisation. 


\section{FICHE PUBLICATION SCIENTIFIQUE}

\section{ARTICLE DE REVUE}

Titre : «Conception Fonctionnelle Intégrant le Calcul Mécanique : Le Prototype F-CAD. »

Auteurs : $\quad$ D. Deneux**, Abdelmajid BenAmara*.

Laboratoires Impliqués :

* Laboratoire de Génie Mécanique (Lab. Ma-05).

** LAMIH, Université de Valenciennes, France.

Nom de Revue / Congrès : $\quad$ REVUE INTERNATIONALE DE CFAO ET D'INFORMATIQUE GRAPHiQUe, VOLUME $13-\mathrm{N}^{\circ} 2 / 1998$.

Etat actuel : $\quad$ Publié 1998.

\section{Résumé détaillé :}

Dans cet article, l'approche proposée pour l'intégration du calcul dans le processus de conception revêt deux aspects : la modélisation du produit et une méthode de conception fonctionnelle. La méthode de conception basée sur les fonctionnalités supporte le processus d'élaboration des concepts qui permet non seulement de structurer les relations entre différents acteurs de la conception, mais aussi de gérer les relations (contraintes) entre les différents concepts, en particulier les relations formes-fonctions. Cette méthode de conception est inspirée des pratiques traditionnelles des bureaux d'études ; elle exige cependant une technique de modélisation de produit différente de celle proposée par les systèmes de CAO commerciaux actuels qui privilégient l'utilisation d'une démarche par essaierreur dans la mesure où la géométrie, qui devrait résulter de l'analyse du problème, est en fait une primitive de modélisation.

Le modèle de produit proposé est constitué d'un modèle de référence commun auquel se réfèrent les applications (en l'occurrence le calcul) pour construire leur propre modélisation. Ce modèle de référence est basé sur des concepts ayant des significations communes pour ces différentes activités et comporte des informations à caractère géométrique (forme) mais aussi technologie (matériaux, tolérances, efforts).

Sa mise en œuvre est basée sur une structure de données objets. Le prototype de système de conception fonctionnelle F-CAD permet de valider la méthode de conception proposée et de monter l'aptitude du modèle du produit à supporter l'intégration des fonctions et des outils relatifs de conception et de calcule mécanique. Dans sa version actuelle, le système ne peut concevoir que des mécanismes simples. La conception de mécanismes plus complexes nécessiterait essentiellement l'enrichissement de la bibliothèque des solutions technologiques et techniques. 


\title{
FICHE PUBLICATION SCIENTIFIQUE
}

\section{ARTiCle DE REVUe}

Titre : «Pré-Dimensionnement en Conception Fonctionnelle »

Auteurs : $\quad$ Abdelmajid BenAmara*, D. Deneux**, R. Soenen**, A. Dogui*.

\author{
Laboratoires Impliqués : \\ * Laboratoire de Génie Mécanique (Lab. Ma-05). \\ ** Département de Génie Mécanique, Université du Québec à Trois Rivières.
}

Nom de Revue / Congrès : Revue internationale de CFAO et d'informatique Graphique ; Vol.11 $n^{\circ} 1-2 / 1996$.

Etat actuel : $\quad$ Publié $1996 .$.

\section{Résumé détaillé :}

Le dimensionnement des pièces mécaniques a depuis longtemps intéressé les concepteurs et a fait l'objet de nombreux travaux de recherche. Dans cet article, on commence par une présentation de l'état de l'art sur les problématiques rencontrées dans ce domaine ainsi que des constatations générales relatives à l'intégration $\mathrm{CAO} /$ Calcul. Nous présentons ensuite la conception basée sur les fonctionnalités pour introduire l'intégration de l'aspect dimensionnement dès la conception. Un modèle d'étude est enfin présenté avant de conclure sur les possibilités d'implémentation et de généralisation de ce travail.

Les principales conclusions de ce papier sont :

La conception par fonctionnalités permet d'intégrer plusieurs aspects d'étude, dont le dimensionnement, dans le même modèle homogène du produit. Ceci constitue l'objet principal autour duquel s'articulent les différents objectifs de l'ingénierie simultanée.

La fonction calcul intervient à plusieurs niveaux du cycle des fonctionnalités et plus particulièrement dans la phase de conception détaillée (depuis le choix des fonctions de base jusqu'à l'étape d'évaluation). Divers types de calculs peuvent apparaître.

En conception fonctionnelle, la fonction calcul sert non seulement pour dimensionner, mais aussi pour choisir les solutions technologiques et techniques ainsi que pour évaluer et optimiser la solution globale adoptée. Notre travail s'est pour l'instant limité au dimensionnement fonctionnel des liaisons inter-pièces en vue d'un pré-dimensionnement global des formes fonctionnelles

Le modèle adopté est basé sur une représentation paramétrée des liaisons inter-pièces ; il permet d'encapsuler les méthodes de dimensionnement dans l'opérateur de construction de chaque type de liaison. Ceci permet d'évaluer les paramètres de la liaison en fonction des contraintes appliquées au système. Nous pouvons donc 
Fiches Communications Nationales et Internationales

$$
\text { (2002 - 2005) }
$$




\section{FICHE PUBLICATION SCIENTIFIQUE}

\section{COMMUNICATION INTERNATIONALE}

Titre : «Reconstruction d'un modèle CAO à partir d'un modèle éléments finis déformé»

Auteurs : $\quad$ Vincent François**, Borhen Louhichi*, Abdelmajid Benamara*

Laboratoires Impliqués :

* Laboratoire de Génie Mécanique (Lab. Ma-05).

** Département de Génie Mécanique, Université du Québec à Trois Rivières.

Nom de Revue / Congrès : 17ème Congrès Français de Mécanique, Troyes Septembre 2005

Etat actuel : Publiée.

\section{Résumé détaillé :}

Dans un contexte d'ingénierie simultanée, l'intégration entre les différentes étapes de modélisation devient de plus en plus une nécessité. En effet, les problèmes d'intégration s'avèrent les premiers responsables de perte de temps durant la phase d'optimisation. Le travail présenté dans ce papier s'inscrit dans le cadre de l'intégration CAO, FAO et Calcul.

Dans ce papier, on commence par la présentation l'état de l'art et l'objectif final de nos travaux de recherche qui consiste à développer un environnement CAO Calcul parfaitement intégré dans le quel on peut simuler que se soit des systèmes mécaniques ou des études élasto plastique en temps réel.

Par la suite on présente notre contribution au développement de cet environnement intégré. Nous proposons une démarche de reconstruction des modèles $\mathrm{CAO}$ à partir des résultats d'une analyse éléments finis dans le cas élastique. L'application au cas élastique permet en réalité de montrer les résultats de l'étude de faisabilité de la démarche globale et complète de reconstruction. La reconstruction dans le cas élastique peut s'appliquer dans la simulation de fonctionnement des systèmes mécaniques dans les conditions de fonctionnement. La démarche de reconstruction dans ce cas consiste à déterminer les nouveaux paramètres (vecteur directeur, point d'insertion...) des différents entités de modèle CAO (faces et arêtes). La connexion de ces différentes entités tout en respectant la topologie initiale permet de reconstruire le modèle CAO déformé. En fin les conclusions et les perspectives de ces travaux sont présentées. 


\section{FICHE PUBLICATION SCIENTIFIQUE}

\section{COMMUNICATION INTERNATIONALE}

Titre : «Intégration numérique et interopérabilité des processus de CAO et de Calcul»

Auteurs : $\quad$ Borhen Louhichi*, Nizar Ifaoui*, Abdelmajid BenAmara*, Vincent francois**, Dominique Deneux***

\section{Laboratoires Impliqués :}

* Laboratoire de Génie Mécanique (Lab. Ma-05).

** Département de Génie Mécanique, Université du Québec à Trois Rivières.

*** LAMIH, LAMIH, UMR CNRS 8530, Université de Valenciennes, France.

Nom de Revue / Congrès : 17ème Congrès Français de Mécanique, Troyes Septembre 2005

Etat actuel : Publiée.

\section{Résumé détaillé :}

De nos jours, nous assistons à un développement du travail en mode projet qui se caractérise par la mobilisation de compétences multiples. La globalisation des marchés ainsi que la réduction des coûts et des délais de développement de nouveaux produits ont conduit à la mise au point d'outils de travail collaboratif et de manière distante tout en assurant la structuration, le suivi, et la traçabilité des échanges. Cela a induit un accroissement considérable des besoins de communication interapplications et de cohérence globale des systèmes supports des différents modèles du produit (CAO, Calcul, FAO).

De part leur forte interdépendances, les deux activités CAO et Calcul seront donc amenées à s'édifier sur de nouvelles technologies émergentes dans les domaines de la modélisation des données (modèle de produit) et des processus (interopérabilité) afin de pouvoir prendre en compte la manipulation d'objets hétérogènes (géométrie, sollicitations, maillage, déformation, etc.). En effet, dans un contexte de travail collaboratif, l'intégration numérique de ces deux activités CAO et Calcul, est devenue une des principales préoccupations en CFAO.

L'objectif recherché est de favoriser le partage des données sans recopies ou transformations manuelles afin de fluidifier les flux d'informations entre $\mathrm{CAO}$ et Calcul tout en garantissant la fiabilité et la traçabilité des données. L'objet de cette communication est de présenter trois principales approches d'intégration numérique et d'interopérabilité des processus de $\mathrm{CAO}$ et de Calcul mécanique développées en collaboration entre le Laboratoire de Génie Mécanique de l'ENI de Monastir (Tunisie), le LAMIH de l'Université de Valenciennes (France) et le Département de Génie Mécanique de l’Université de Québec à Trois Rivières (Canada). 


\section{FICHE PUBLICATION SCIENTIFIQUE}

\section{COMMUNICATION INTERNATIONALE}

\begin{tabular}{|c|c|}
\hline Titre : & $\begin{array}{c}\text { "Rebuilding deformed CAD model starting FEA results" } \\
\text { "Elastic linear case study" }\end{array}$ \\
\hline Auteurs : & $\begin{array}{l}\text { Borhen Louhichi*, Abdelmajid BenAmara*, Vincent François**, } \\
\text { Lotfi Romdhane* }\end{array}$ \\
\hline Laboratoi & $\begin{array}{l}\text { Impliqués : } \\
\text { * Laboratoire de Génie Mécanique (Lab. Ma-05). } \\
\text { ** Département de Génie Mécanique, Université du Québec à Trois Rivières. }\end{array}$ \\
\hline
\end{tabular}

Nom de Revue / Congrès : CANCAM 2005, Université MacGill, Montréal le 2 juin 2005.

\section{Etat actuel : $\quad$ Publiée}

\section{Résumé détaillé :}

Dans ce papier une partie de notre activité de recherche, qui s'articule autour de l'intégration de la méthode des éléments finis dans le processus $\mathrm{CAO} / \mathrm{FAO}$ par reconstruction du modèle $\mathrm{CAO}$ à partir des résultats éléments finis (Maillage déformé), est présentée. Cette partie est focalisée sur la reconstruction du modèle $\mathrm{CAO}$ dans le cas où le modèle a subit des changements géométriques mais pas des changements topologiques, ce cas peut être appliquer pour les pièces en flexion.

$\mathrm{Au}$ début l'algorithme général de reconstruction est présenté. Cet algorithme se défère suivant le déformations en d'autres termes suivant la nature des changement topologiques et géométriques de modèle. On caractérise trois cas possibles :

- Ni changements topologiques, ni changements géométriques de modèle.

- Changements géométriques sans changements topologiques.

- Changements topologiques et changements géométriques.

Ce papier traite le deuxième cas, dans le quel les natures des faces et arêtes changent. Dans ce cas, la phase la plus difficile est la reconstruction des surfaces porteuses des faces déformées. Un algorithme de reconstruction d'une surface à partir de la triangulation issue d'un maillage a été présenté. Et des cas d'application sont traités.

En fin, les conclusions et les perspectives de nos travaux sont présentées.
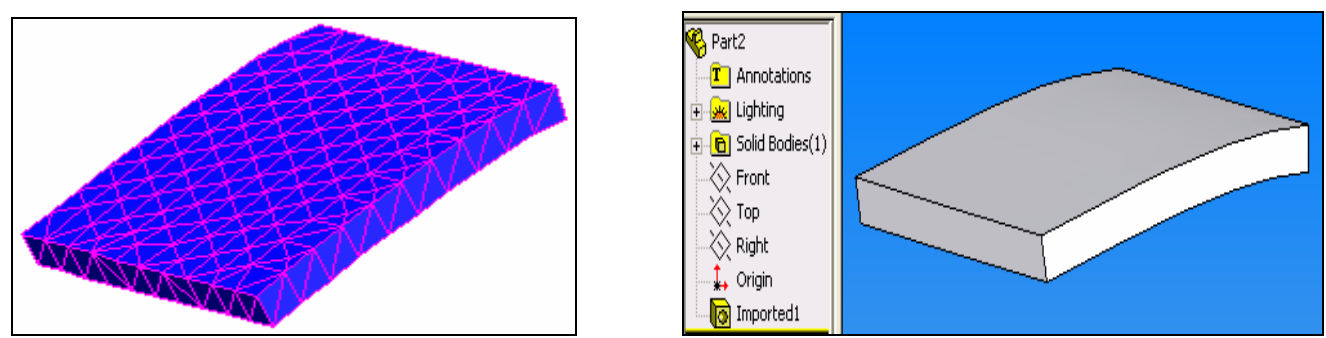

Reconstruction d'un modèle BREP à partir du maillage déformé. 


\section{FICHE PUBLICATION SCIENTIFIQUE}

\section{COMMUNICATION INTERNATIONALE}

Titre : «Intégration de la méthode des éléments finis dans le processus de CAO : détermination de la géométrie d'un modèle déformé.»

Auteurs : $\quad$ Borhen Louhichi*, Vincent François**, Abdelmajid Benamara *

\section{Laboratoires Impliqués :}

* Laboratoire de Génie Mécanique (Lab. Ma-05).

** Département de Génie Mécanique, Université du Québec à Trois Rivières.

Nom de Revue / Congrès : $\quad$ 73e Congrès de l'ACFAS, Université du Québec à Chicoutimi.

Etat actuel : Publiée le 12 mai 2005

\section{Résumé détaillé :}

Dans le cadre de notre projet de recherche concernant l'intégration de la méthode des éléments finis au sein de la Conception Assistée par Ordinateur, différents travaux sont réalisés. Parmi ceux-ci, la détermination du modèle $\mathrm{CAO}$ de la déformée d'un objet obtenue par simulation numérique est une préoccupation importante. Le modèle CAO est de type BREP (Boundary REPresentation). Dans ce modèle il y a deux types d'informations qui permettent de décrire les frontières du modèle :

- L'information géométrique: Cette information permet de définir les propriétés de chacune des entités de modèle BREP (les faces et les arêtes). Par exemple une face plane, arête circulaire... sont tous des informations géométriques.

- L'information topologique : Cette information décrit l'arrangement et les liens entre les différentes entités.

Dans cette communication, nous montrons la manière employée pour retrouver l'information géométrique qui consiste à modéliser les frontières du modèle BREP par des NURBS (Non Uniform Rational B-Spline) qui sont les surfaces porteuses des faces de ce dernier. Le choix de NURBS vient de fait qu'une surface déformée est de nature quelconque généralisé par ce type de surface.

la présentation commence par un positionnement de problème général, puis un algorithme qui détermine l'information géométrique est présenté. Enfin, les autres points à résoudre sont décrits, pour converger vers la solution complète de la reconstruction du modèle BREP d'un solide déformé par la simulation numérique. 


\section{FICHE PUBLICATION SCIENTIFIQUE}

\section{COMMUNICATION INTERNATIONALE}

Titre : « Reconstruction d'un modèle BREP déformé à partir des résultats d'une simulation éléments finis»

Auteurs : $\quad$ Borhen Louhichi*, Abdelmajid BenAmara*, Vincent François**

Laboratoires Impliqués :

* Laboratoire de Génie Mécanique (Lab. Ma-05).

** Département de Génie Mécanique, Université du Québec à Trois Rivières.

Nom de Revue / Congrès : $\quad$ Premier Congrès International Conception et Modélisation des Systèmes Mécaniques, Hammamet, 23-25 mars 2005

Etat actuel : $\quad$ Publié.

\section{Résumé détaillé :}

Pendant longtemps, l'utilisation de la simulation numérique par la méthode des éléments finis a été complètement découplée du processus de CAO (Conception Assistée par Ordinateur). Sa phase de saisie des données était longue et fastidieuse. Comme, elle nécessite également de grosse capacité informatique, sa mise en oeuvre était rare et coûteuse. Depuis la progression fulgurante des systèmes informatiques, son utilisation est devenue de plus en plus abordable si bien que les utilisateurs ont ressenti le besoin d'une amélioration de l'ensemble du processus et particulièrement la phase de saisie des données. Celle-ci consiste à construire un support de calcul qui est constitué d'une juxtaposition parfaite de formes géométriques simples (ligne, triangle, quadrangle, tétraèdre, hexaèdre) appelées maillage. Des conditions aux limites et des chargements sont ensuite positionnées sur ce maillage. L'idée d'utiliser les outils performants issus de la CAO pour réaliser plus efficacement la phase de saisie est naturelle. En effet toutes les fonctions nécessaires à cette phase sont présentes dans les logiciels de CAO. C'est ainsi qu'au cours de ces dernières années, plusieurs solutions sont apparues pour créer une interaction entre le monde de la $\mathrm{CAO}$ et celui de la simulation numérique.

Cependant, dans un premier temps, un simple couplage a été réalisé c'est à dire qu'un modèle éléments finis est construit à partir d'un modèle CAO pour constituer deux modèles distincts qui évoluent de manière indépendante par la suite. Bien qu'étant un énorme progrès, cette situation n'est pas idéale dans le contexte de l'ingénierie simultanée. En effet les modèles sont susceptibles d'être modifiés lors de n'importe quelle phase de la réalisation d'un produit. Il est bon dans ce cas de conserver une certaine intégrité entre toutes les données nécessaires à la réalisation d'un produit. $\mathrm{Au}$ fil du temps, nos travaux consistent à réaliser de nouvelles fonctions qui permettent à notre modèle de converger vers un modèle parfaitement intégré avec la CAO. Ce papier présente une de ces fonctions qui est la méthode de reconstruction d'un modèle solide déformé à partir des résultats d'une simulation par éléments finis. 


\begin{tabular}{|c|c|}
\hline \multicolumn{2}{|r|}{ FICHE PUBLICATION SCIENTIFIQUE } \\
\hline \multicolumn{2}{|r|}{ COMMUNICATION AFFICHEE « POSTER » } \\
\hline \multicolumn{2}{|c|}{$\begin{aligned} \text { Titre : } & \text { " Etude expérimentale de l'influence des paramètres de coupe sur l'état de surface } \\
& \text { en tournage dur }\end{aligned}$} \\
\hline \multicolumn{2}{|c|}{$\begin{array}{ll}\text { Auteurs : } & \text { Imed Hajjaji, } \\
& \text { Abdelmajid BenAmara, } \\
& \text { Fredj Ramzi }\end{array}$} \\
\hline Laboratoires Impliqués : & Laboratoire de Génie Mécanique, ENIM \\
\hline Nom de Revue / Congrès : & $\begin{array}{l}\text { Premier Congrès International Conception et Modélisation des } \\
\text { Systèmes Mécaniques, Hammamet, 23-25 mars } 2005 .\end{array}$ \\
\hline Etat actuel : & Publié. \\
\hline
\end{tabular}

\section{Résumé détaillé :}

Le tournage dur, est un procède récent dans son utilisation. IL se présente comme une alternative à la rectification conventionnelle, souvent longue et coûteuse lors de l'usinage des pièces de précision. C'est un procédé promoteur qui intéresse de nombreux industriels pour les avantages qu'il présente du point de vue productivité et flexibilité.

Afin de maîtriser le procédé du tournage dur et de rentabiliser son utilisation industrielle il est nécessaire d'étudier la qualité de la surface obtenue ainsi que les lois qui régissent sa variation en fonction des paramètres d'usinage.

Plusieurs paramètres entre en jeu pour caractériser la qualité de la surface obtenue en tournage dur, tels que les paramètres de coupe (vitesse de coupe, vitesse d'avance, et la profondeur de passe) ; les paramètres de l'outil : rayon du bec, matériau, ainsi que les caractéristiques du matériau usiné (dureté, état métallurgique...).

Cette communication représente une étude bibliographique sur les facteurs influençant l'état de surface en tournage dur ainsi que une étude expérimentale de l'usinage de finition de l'acier $\mathrm{X} 155 \mathrm{CrMoV} 12$ durcis à $56 \mathrm{HRC}$ avec un outil c-BN afin d'étudier l'impact des paramètres de coupe sur la rugosité de surface obtenu par tournage dur.

Un modèle de prédiction de la qualité de la surface en tournage dur en fonction des paramètres d'usinage est établi en appliquant la méthode du plan d'expériences. Ce modèle permet l'optimisation du choix des paramètres de coupe en tenant en compte la rugosité de la surface désirée. 


\section{FICHE PUBLICATION SCIENTIFIQUE}

\section{COMMUNICATION NATIONALE}

Titre : «Aperçu des techniques de prédiction des états des surfaces usinées.»

Auteurs : Ramzi FREDJ ; ABDELMAJID BENAMARA

Laboratoires Impliqués :

* Laboratoire de Génie Mécanique (Lab. Ma-05).

\section{Nom de Revue / Congrès :}

Premier Congrès International Conception et Modélisation des Systèmes Mécaniques CMSM'2005, Hammamet, Tunisie, mars 2005

\section{Etat actuel : Publiée.}

\section{Résumé:}

L'accroissement des performances des machines outils et des outils de coupe a permis d'accroître considérablement la productivité des procédés d'usinage tout en améliorant la qualité des surfaces mises en forme. Cependant, la prédiction de la qualité d'usinage à partir des conditions d'obtention, des équipements utilisés et des contraintes du temps et de coût, reste un problème majeur dans la fabrication. $\mathrm{Vu}$ les nombreux paramètres qui influent la rugosité, sa prédiction reste parmi les principales difficultés rencontrées.

Ce papier présente les différentes techniques et approches utilisées dans l'évaluation de l'état de surface. Trois classes d'approches sont étudiées : théorie des machines, analyse expérimentale et application de l'intelligence artificielle. Vu leur rôle important, les modèles de détermination des efforts de coupe et de déformation de l'outil en fraisage sont présentés par leurs principes.

Dans ce travail nous avons décrit un ensemble des techniques adoptées par des chercheurs en vue de prédire l'état de surface usinée. Il est évident d'après les publications consultées, que le sujet est riche et un grand effort de recherche est développé dans ce domaine. Toutes les méthodes ont des avantages avec des limites d'emploi. D'après [Benardos 2002] celles qui présentent un avenir prometteur sont les modèles analytiques et l'intelligence artificielle.

L'approche de l'intelligence artificielle permet a priori d'avoir le système de prédiction le plus réaliste. Elle présente l'avantage d'une intégration informatique relativement facile à réaliser et elle peut être utilisé avec d'autres méthodes expérimentales ou analytiques. Un autre avantage c'est son adaptation à traiter des problèmes d'optimisation comme l'algorithme génétique. Ces avantages le place à mon avis en premier plan dans le cas où on vise le pilotage de processus d'usinage à travers une prédiction de la rugosité ou autre grandeur comme l'usure.

Néanmoins, une méthode hybride qui utilise conjointement la méthode analytique et l'intelligence artificielle en exploitant leurs avantages sera sûrement intéressante pour tenir compte de plusieurs paramètres et avoir un calcul économique. Pour chaque approche, des études typiques sont sélectionnées : les grandes lignes sont présentées avec leurs avantages, leurs limites d'emploi et la précision des résultats obtenus. Les exploitations réalisées et les applications possibles ont été vues et commentées. Une analyse des tendances avec une discussion suivie d'une conclusion a fait l'objet de la dernière partie de cette communication. 


\section{FICHE PUBLICATION SCIENTIFIQUE}

\section{COMMUNICATION INTERNATIONALE}

Titre : « Les Features de Calcul : Un modèle générique pour l'interopérabilité CAO-Calcul »

\begin{tabular}{ll}
\hline Auteurs : & N. AIFAOUI* — A. BENAMARA* — D. Deneux** \\
\hline Laboratoires Impliqués : & * Laboratoire de Génie Mécanique (Lab. Ma-05). \\
& $* *$ LAMIH, LAMIH, UMR CNRS 8530, Université de Valenciennes, France.
\end{tabular}

Nom de Revue / Congrès : CMSM 2005, Hammamet Tunisie

Etat actuel : $\quad$ Publié en Mars 2005

\section{Résumé détaillé :}

Dans cet article une approche originale d'intégration $\mathrm{CAO} / \mathrm{Calcul}$ a été introduite. Cette approche est basée sur le concept de feature de calcul. Ce concept joue le rôle d'un vecteur de communication entre le domaine de conception et de calcul, et favorise ainsi la coopération dans l'accomplissement des tâches tout au long du processus de conception.

Le concept de Feature de calcul a été modélisé par un sextuplet (3 modèles de données, 3 relations de cohérence) globalement adapté à la caractérisation et à la résolution des problèmes de calcul typiques couramment rencontrés en conception mécanique. Un catalogue de features a permis de structurer un nombre restreint de features de calcul particulières. Pour avoir une représentation normalisée, le formalisme UML a été utilisé pour décrire le modèle de données du catalogue ainsi que les processus de choix et d'instanciation de features à partir du catalogue. La validation de la méthode a porté sur la conception d'un système de fermeture d'une machine à mouler.

Dans cet article une approche originale d'intégration $\mathrm{CAO} / \mathrm{Calcul}$ a été introduite. Cette approche est basée sur le concept de feature de calcul. Ce concept a été modélisé par un sextuplet (3 modèles de données, 3 relations de cohérence) globalement adapté à la caractérisation et à la résolution des problèmes de calcul typiques couramment rencontrés en conception mécanique. Un catalogue de features a permis de structurer un nombre restreint de features de calcul particulières. Pour avoir une représentation normalisée, le formalisme UML a été utilisé pour décrire le modèle de données du catalogue ainsi que les processus de choix et d'instanciation de features à partir du catalogue. La validation de la méthode a porté sur la conception d'un système de fermeture d'une machine à mouler. 


\section{FICHE PUBLICATION SCIENTIFIQUE}

\section{COMMUNICATION INTERNATIONALE}

Titre : «Simulation cinématique d'un assemblage dans les conditions de fonctionnement.»

Auteurs : $\quad$ Borhen Louhichi*, Abdelmajid Benamara*, Vincent François **

\section{Laboratoires Impliqués :}

* Laboratoire de Génie Mécanique (Lab. Ma-05).

** Département de Génie Mécanique, Université du Québec à Trois Rivières.

Nom de Revue / Congrès : 72e Congrès de l'ACFAS, Université du Québec à Montréal.

Etat actuel : $\quad$ Publiée le 10 Mai 2004.

\section{Résumé:}

Depuis plusieurs années, l'étude de l'intégration de la méthode des éléments finis dans le processus de CAO/FAO permet de développer de nouveaux outils. Dans ce cadre, notre préoccupation actuelle est de construire une procédure de reconstruction d'un modèle solide de type BREP à partir des résultats d'une analyse numérique par éléments finis qui est le maillage déformé. Nos travaux sont divisés en plusieurs phases suivant les déformations engendrés sur le modèle.

- La première qui est maintenant complétée consiste à développer la procédure dans le cas des études purement élastiques, là où les déformations engendrées sur le modèle sont très faibles. Donc les informations topologiques et géométriques sur le modèle CAO restent les mêmes avant et après déformation.

- La deuxième partie traite le cas où les déformations que se soit élastiques où inhérentes engendrés sur le modèle provoquent des changements géométriques sur ce dernier tout en gardant la même topologie (le nombre et les liens entres les différentes entités géométriques et topologiques restent les mêmes avant et après déformation).

- La troisième partie traite le cas des grandes déformations qui engendrent sur le modèle des changements géométriques et topologiques. Dans ce cas le modèle Final est totalement différent de l'initial. Une recherche de la topologie est nécessaire dans cette partie, avant la reconstruction des entités du modèle.

Dans cette communication, on s'intéresse à la première partie tout en montrant l'intérêt de la reconstruction dans ce cas. Une application pratique de la procédure est la simulation cinématique d'un assemblage. Dans tous les systèmes de $\mathrm{CAO}$, cette simulation est réalisée avec des corps rigides non contraints. Cette hypothèse est satisfaisante dans la plupart des cas. Cependant dans quelques cas, il serait souhaitable d'effectuer la simulation en tenant compte de l'état contraint des pièces. C'est la démarche utilisée pour réaliser ce dernier point qui constitue le thème de cette communication. Ainsi, notre exposé commence avec un bref rappel de notre thème de recherche dans son ensemble. Le projet global de reconstruction de modèle solide déformé est ensuite expliqué. L'application à la simulation cinématique d'un assemblage bielle manivelle illustre les résultats. 


\section{FICHE PUBLICATION SCIENTIFIQUE}

\section{COMMUNICATION NATIONALE}

Titre : «Optimisation des plans de matelassage à l’aide des algorithmes génétiques.»

\begin{tabular}{|ll|}
\hline Auteurs : & $\begin{array}{l}* \text { WISSEM GUENANNA ; *ABDELMAJID BENAMARA ; } \\
* * \text { FAOUZI SAKLI }\end{array}$ \\
\hline $\begin{array}{ll}\text { Laboratoires Impliqués : } \\
* \text { Laboratoire de Génie Mécanique (Lab. Ma-05). } \\
* * \text { Unité de Recherches Textiles, ISET Ksar Helal. }\end{array}$ \\
\hline Nom de Revue / Congrès : & $\begin{array}{l}\text { The First International Conference of Applied Research in } \\
\text { Textile ; CIRAT ; pages 143-150, Monastir (2004). }\end{array}$ \\
\hline Etat actuel : & Publiée. \\
\hline
\end{tabular}

\section{Résumé:}

Le traitement des plans de matelassage est une étape déterminante qui permet de générer des économies de matière et des gains de temps énormes dans l'industrie de la confection. La recherche opérationnelle s'est intéressée à des problèmes similaires dans des domaines très variés. Nous avons élaboré par le biais de ce travail un nouvel outil d'optimisation des plans de matelassage qui consiste à développer une base diversifiée de solutions possibles à un problème de matelassage et ensuite en appliquer un certain nombre d'opérateurs génétiques jusqu'à extraire la meilleure solution. La particularité de la méthode proposée c'est que le critère de sélection est le nombre de matelas par solution proposée et le nombre d'articles produits par chaque taille et pour chaque solution est identique à celui de la commande initiale. Les algorithmes trouvés ont été testés et le résultat est satisfaisant quant à la réalisation d'un plan de matelassage dans un temps réduit bien que l'affinage dépend de la complexité du problème.

Le problème de plan de matelassage entre dans le cadre des problèmes d'optimisation et de recherche opérationnelle qui traitent assez souvent des problèmes similaires mais dans d'autres domaines comme la papeterie, la métallurgie etc. Les recherches qui traitent directement le placement et le matelassage dans la confection et prennent en considération toutes leurs spécificités sont assez rares et très récentes. Parmi ces recherches on peut citer le travail réalisé par Z.degraeve qui propose de résoudre le problème du plan des matelassage par deux méthodes qui sont le NLIP (non linear integer programming) et une deuxièmes méthode qui consiste à linéariser ce premier modèle pour faciliter la réalisation des algorithmes et minimiser le temps de calcul [3]. Ces deux méthodes ne sont applicables que dans le cas de commandes réduites et d'un nombre de tailles très limité. Une troisième méthode assez récente a été proposée par J. Martens et qui consiste à concevoir deux algorithmes génétiques qui peuvent traiter des problèmes de dimensions plus larges [4].

La méthode proposée pour résoudre le problème de plan de matelassage en utilisant les algorithmes génétiques est une méthode adaptée à ce type de problèmes. La validation des algorithmes proposés a donné des résultats satisfaisants et dans la plupart des exemples traités on arrive a atteindre une solution optimale. Dans quelques cas, le programme peut stagner sur des solutions non optimales et c'est normal puisque le hasard est le phénomène le plus dominant dans le processus d'optimisation de ces algorithmes, mais on peut encore améliorer l'opérateur de sélection pour éviter les phénomènes de stagnation sur des minimums locaux. 


\section{FICHE PUBLICATION SCIENTIFIQUE}

\section{COMMUNICATION INTERNATIONALE}

Titre : « Toward a management model of design and mechanical analysis processes based on feature concept"

\begin{tabular}{ll|}
\hline Auteurs : & N. Aifaoui*, D. Deneux \\
\hline Laboratoires Impliqués : \\
* Laboratoire de Génie Mécanique (Lab. Ma-05). \\
\\
$\begin{aligned} * * \text { LAMIH, LAMIH, UMR CNRS 8530, Université de Valenciennes, France. } \\
* * * \text { PRISMA : INSA-Lyon, France }\end{aligned}$ \\
\hline Nom de Revue / Congrès : & IDMME 04, Bath Angleterre \\
\hline Etat actuel : & Publié en Avril 2004 \\
\hline
\end{tabular}

\section{Extended Abstract :}

In this paper, we firstly intend to clearly position our research objectives against the huge amount of works related to CAD-Analysis integration. Our main research goal was to emphasize the existence of generic and modular tasks in the various loops between design and analysis (dimensioning or evaluation) processes.

In this paper, a new method has been introduced, allowing a better integration between the parallel processes of design and analysis. This method is based on the original concept of analysis feature. The concept of analysis features can be formally defined by sextuple involving three data models and three coherence relations. This formalization results from an experimental study of mechanical design cases, involving several typical dimensioning and evaluation problems involving static, kinematics and dynamic analysis. Based on this experimental study, the structure and initial contents of a features catalogue has been defined.

The proposed approach permits to solve several analysis problems (limited to "solid mechanics field") usually encountered in the design of mechanical systems, in particular during the early phases of design. It can provide tracability to the analysis loops, fostering the re-use of existing analysis and reducing the time and effort required to perform this trial and error process.

Although there is currently no working prototype, the implementation issue has been considered. The specification of the feature-based interactive design-and-analysis system has been prepared using the UML formalisms. Thus, the catalogue-class diagram, the main usercases, and the feature selection and definition processes have been detailed. 


\section{FICHE PUBLICATION SCIENTIFIQUE}

\section{COMMUNiCATION NATIONALE}

Titre : Etude Expérimentale et Numérique de l'essaie de gonflement hydraulique.»

Auteurs : Abdessalem Chamekh*,

Amen GAhbiche*,

Hedi Belhadj Salah*

Abdelmajid BenAmara*

Abdelwaheb Dogui*

Laboratoires Impliqués : $\quad$ * Laboratoire de Génie Mécanique (Lab. Ma-05).

Nom de Revue / Congrès : $\quad$ Quatrièmes Journées Scientifiques JS'2003, 21-22 mai 2003, Ecole de l'Aviation de Borj El Amri, Tunisie"

Etat actuel : $\quad$ Publiée mai 2003.

Résumé:

La mise en forme par déformation plastique des tôles métalliques continue à être une activité industrielle importante. A titre d'exemple, l'emboutissage représente de 15 à $20 \%$ du coût d'étude et de développement d'un nouveau véhicule [MOU02]. A ce niveau, la simulation numérique des procédés est un outil très important. Elle permet de tester, de comparer et de dimensionner ces procédés à faible coût surtout avec les développements récents des outils de calcul non linéaire des structures. Mais la qualité de telles simulations dépend fortement de celle de la compréhension et de la description du comportement des matériaux déformés. Pour la mise en forme à froid, le comportement des tôles est élastoplastique anisotrope en grandes déformations. L'identification de tels modèles de comportement à partir des essais expérimentaux continue à présenter des difficultés. En effet, bien que l'essai de traction simple reste, pour les matériaux solides, la référence, il ne suffit plus pour identifier le comportement de modèles de plus en plus raffinés et des informations complémentaires doivent parvenir d'autres essais [BAR02].

Nous présentons dans ce travail des simulations numériques de l'essai d'expansion par gonflement hydraulique. Cet essai à l'avantage de se dérouler presque sans frottement ce qui rend la simulation du comportement de la tôle au cours de sa mise en forme, a priori, plus facile.

Nous présentons l'essai expérimental au second paragraphe de ce papier. Le troisième paragraphe est dédié aux modèles considérés dans les simulations présentées. Nous présentons au quatrième paragraphe les résultats numériques ainsi que leurs analyses et interprétations. 


\section{FICHE PUBLICATION SCIENTIFIQUE}

\section{COMMUNICATION INTERNATIONALE}

Titre : "Mechanical analysis process modeling based on analysis features "

Auteurs : $\quad$ N. Aifaoui*, D. Deneux**, A. Benamara*, R. Soenen***, A. Dogui*,

Laboratoires Impliqués :

* Laboratoire de Génie Mécanique (Lab. Ma-05).

** LAMIH, LAMIH, UMR CNRS 8530, Université de Valenciennes, France.

*** PRISMA : INSA-Lyon, France

Nom de Revue / Congrès : $\quad$ IEEE, Hammamet, Tunisie

Etat actuel : $\quad$ Publié en Octobre 2002

\section{Extended Abstract :}

Today, industrial products are so complex that they require a synergy of experts, assisting the designer by analyzing and validating design assumptions, under a variety of heterogeneous constraints derived from product life cycle viewpoints. Mechanical analysis belongs to this kind of expertise. It is focused on the validation and optimization of the mechanical properties of the product. The designer and the mechanical expert concurrently aim at a common goal which is a good product, satisfying a proven need. But each actor has his own perception of the product, manages different models, makes use of different methods.

In this paper we have presented an original approach for a better integration of design and mechanical analysis process. The method proposed is based on the notion of analysis features, and is dedicated to help a designer, non expert in mechanical analysis, to characterize and implement such analysis on his own, in particular to perform pre-dimensioning or evaluate his design propositions.

Analysis features allow the modelling and resolution of a typical analysis problems usually encountered in design processes. An experimental study of design examples allowed us to characterize six generic features classes. Analysis features classes are modular and generic processes, adaptable to a variety of specific design contexts.

This approach also increases the tracability of the analysis process and the re-use of analysis paths after slight design modifications.

The future work will be concentred on the implementation of the proposed method in a computer system. 


\section{FICHE PUBLICATION SCIENTIFIQUE}

\section{COMMUNICATION NATIONALE}

Titre : « Modèle de conception basé sur les entités technologiques.»

\begin{tabular}{|l|l|}
\hline Auteurs : & $\begin{array}{l}\text { FATHI BOUABDA } \\
\text { ABDELMAJID BENAMARA }\end{array}$ \\
\hline $\begin{array}{c}\text { Laboratoires Impliqués : } \\
\text { * Laboratoire de Génie Mécanique (Lab. Ma-05). }\end{array}$ \\
\hline Nom de Revue / Congrès : $\quad \begin{array}{l}\text { Le Premier Colloque de Recherche Appliquée et de Transfert } \\
\text { de Technologie, Radès, Tunisie } 11-12 \text { décembre } 2002 .\end{array}$ \\
\hline Etat actuel : $\quad$ Publiée déc 2002. \\
\hline
\end{tabular}

\section{Résumé:}

La nouvelle démarche d'ingénierie concourante, justifiée et assistée par la vertigineuse avancée des moyens et outils informatiques et par la course effrénée à la qualité des produits, a orienté les industriels et les chercheurs vers l'intégration des différentes activités du cycle de vie d'un produit mécanique. Cette nouvelle tendance exige non seulement la communication entre les outils informatiques, supports des différentes activités de conception et de fabrication, mais aussi la prise en compte des points de vue multiples et des contraintes spécifiques à chaque métier. Dans un tel contexte, il est nécessaire d'éviter au maximum les allers et retours entre les différentes phases de modélisation du produit qui sont les causes principales de perte de temps.

Ce papier présente un modèle de conception basé sur les entités technologiques permettant de favoriser l'intégration des activités de conception et de fabrication des moules d'injection plastique. Ce modèle repose sur l'intégration des informations géométriques (morphologies et dimensions) et technologiques (matériau, tolérances, états de surfaces, procédés d'usinage, ...) dans une même structure de donnée dite modèle de produit. Nous avons adopté le concept générique FEATURE «entité technologique » comme support d'informations provenant des différents intervenants du processus de conception. Ce support d'information est ensuite exploité par les différents modules de l'outil de conception pour générer les informations requises.

En début de ce papier nous présentons un état de l'art permettant d'éclairer les principaux modèles proposés dans la littérature suivi d'une synthèse bibliographique permettant de conclure sur les approches présentées. Nous introduisant ensuite le modèle de conception proposé et nous détaillons sa structure basée sur une approche orientée objet en s'appuyant sur les modèles de représentation graphiques OMT.

L'application de ce modèle à la conception des moules d'injection plastique fera l'objet de la troisième partie de ce papier. En vue d'illustrer l'apport du modèle proposé, nous présentons en fin la maquette informatique développée sous l'environnement orienté objet OpenCascade suivie de nos conclusions et perspectives. 


\section{FICHE PUBLICATION SCIENTIFIQUE}

\section{COMMUNICATION INTERNATIONALE}

Titre : «Intégration CAO/Analyse autour d'un modèle générique du processus de calcul mécanique "

Auteurs : $\quad$ N. Aifaoui*, D. Deneux**, A. BenAmara*

Laboratoires Impliqués :

* Laboratoire de Génie Mécanique (Lab. Ma-05).

** LAMIH, LAMIH, UMR CNRS 8530, Université de Valenciennes, France.

Nom de Revue / Congrès : IDMME 02, Clermont-Ferrand, France

Etat actuel : $\quad$ Publié en Mai 2002

\section{Résumé détaillé :}

Dans ce papier, nous avons présenté une approche permettant de prendre en compte le calcul de comportement au cours de la conception. Nous avons commencé par introduire notre méthodologie de recherche, qui comporte deux volets. D'une part, un état de l'art sur les principaux travaux effectués dans le domaine de l'intégration $\mathrm{CAO} / \mathrm{Calcul}$, qui a permis d'identifier la problématique de recherche et de positionner nos objectifs d'intégration. D'autre part, une étude de cas de conception, qui nous a aidé à la construction d'une typologie de comportements mécaniques auxquels doit répondre un produit, et à l'identification d'un processus générique de calcul d'évaluation des comportements mécaniques tout au long de la conception.

L'étude effectuée a montré que lors de la conception, généralement, le concepteur cherche à satisfaire des exigences fonctionnelles de comportement mécanique. Nous avons défini trois exigences fonctionnelles de comportement (exigence de résistance, exigence de mouvement et exigence de positionnement). Pour satisfaire ces exigences, nous avons introduit un modèle, qui permet de supporter le processus d'analyse mécanique au cours de la conception.

Le concepteur commence par identifier le besoin en calcul de dimensionnement et/ou d'évaluation à partir des solutions de conception. Ensuite, il cherche à modéliser le comportement le système et l'effet de son environnement en spécifiant un certain nombre d'hypothèses qui portent sur les entités : structure, forme, sollicitations, matériau et effets à observer pour évaluer le comportement mécanique du système. Puis, un modèle de simulation approprié aux hypothèses adoptées permet de mettre en œuvre le calcul et fournir les critères d'interprétation permettant au concepteur d'analyser et de juger le comportement du système vis à vis des objectifs de conception. 


\section{ANNEXE II :}

Fiches Encadrement Scientifique 
Fiches Encadrement Thèses de Doctorat 


\section{FICHE ENCADREMENT SCIENTIFIQUE}

\section{THESE DE DOCTORAT}

Titre : «Intégration $\mathrm{CAO} / \mathrm{Calcul}$ par reconstruction du modèle $\mathrm{CAO}$ à partir des résultats éléments finis»

Financement / Formule : Cotutelle, Séjours alternés. Plein temps avec financement.

Période : Démarrage septembre 99 - Soutenue 03 Juillet 2003.

Laboratoires Impliqués :

* Laboratoire de Génie Mécanique (Lab. Ma-05).

** LAMIH, LAMIH, UMR CNRS 8530, Université de Valenciennes, France..

Encadreurs : René Soenen**

Dominique Deneux**

Abdelwaheb Dogui*

Abdelmajid BenAmara*

Etudiant : $\quad$ Ifaoui Nizar

\section{Présentation :}

De nos jours, les produits techniques sont de plus en plus complexes et nécessitent la participation de plusieurs acteurs (mécanicien, automaticien, électricien, maintenicien...). Ces différents acteurs doivent travailler de façon concourante et simultanée sur le même produit à partir de modèles communs (les spécifications des besoin et des exigences fonctionnelles) ou spécifiques (modèle d'assemblage, modèle de commande, modèle électrique, modèle de pannes...). Dans le cadre de cette thèse, nous nous sommes intéressé particulièrement au rôle du concepteur mécanicien, et notre attention se porte donc sur la prise en compte de l'activité de calcul mécanique au cours de la conception. Plus précisément, notre objectif est de proposer et valider une formalisation de processus génériques de calcul utilisables dans différents contextes de conception, afin de guider le concepteur mécanicien dans le déroulement de son activité.

Dans le cadre de cette thèse une approche originale permettant un cadre général d'interopérabilité entre les processus parallèles de conception et de calcul a été introduite. Cette approche est basée sur le concept de feature de calcul. Ce concept fait référence à 3 modèles de données et 3 relations de cohérence entre ces modèles. Ce concept a émergé suite à une étude expérimentale du processus conception-calcul qui a permit d'identifier des problèmes de calcul typiques à partir d'une analyse de plusieurs cas de conception. Un catalogue de features a permis de structurer un nombre restreint de features de calcul particulières. Pour avoir une représentation normalisée, le formalisme UML a été utilisé pour décrire le modèle de données du catalogue ainsi que les processus de choix et d'instanciation de features à partir du catalogue. La validation de la méthode a porté sur la conception d'un système de fermeture d'une machine à mouler.

\section{Résultats :}

\section{Modèle proposé : Les features de calcul mécanique}

Dans notre démarche nous proposons d'associer à chaque classe de problème de calcul une classe de feature de calcul. D'après la généralisation des processus et des modèles de calcul effectuée dans le précédent chapitre, les features de calcul doivent être capables de modéliser de manière cohérente les modèles d'interprétation, les hypothèses (structure, forme, sollicitations, caractéristiques matérielles) et les modèles de simulation qui caractérisent le processus de modélisation et de résolution des problèmes de calcul. 
Une combinaison particulière entre un modèle d'interprétation, des hypothèses et un modèle de simulation représente alors une feature de calcul particulière, caractérisant un problème de calcul particulier dans un niveau de modélisation de produit donné.

Dans cette section nous commençons par définir la notion de feature de calcul dans un contexte de conception intégré. Ensuite, nous donnons le modèle formel de la feature de calcul indépendamment des tâches de calcul et du niveau de modélisation produit. Enfin, et en nous basant sur les cas d'étude, nous détaillons au niveau de définition technologique les classes de features de calcul correspondant aux classes de problèmes de calcul identifiées dans l'étude de cas.

\section{Définition de la Feature de Calcul}

Notre définition de la feature de calcul est inspirée de la définition généralisée de [Shah 91]. Nous définissons la feature de calcul comme "une entité générique paramétrée caractérisant une classe de calcul mécanique ».

L'objet des features de calcul est de représenter le point de vue du mécanicien au cours du processus de conception. Elles représentent des primitives méthodologiques de conception permettant de transformer les problèmes rencontrés en conception (dont la résolution nécessite des tâches de calcul) en des problèmes de calcul, puis à supporter la mise en œuvre du calcul en assistant le concepteur dans:

- Le choix du modèle d'interprétation du comportement du produit

- Le choix des hypothèses de modélisation du produit vis à vis de son environnement

- Le choix d'un modèle de simulation pour estimer ou évaluer le comportement du produit.

Dans la section suivante nous détaillons le modèle formel de la feature de calcul (FC).

Modèle formel de la feature de calcul (FC)

\begin{tabular}{|c|c|c|}
\hline Ensembles d'informations & Définition & Modèle de représentation \\
\hline $\begin{array}{l}\text { Modèle d'Interprétation } \\
\qquad<\mathrm{MI}>\end{array}$ & $\begin{array}{l}\text { Ensemble de variables de conception qui } \\
\text { représentent des points de vue particuliers du } \\
\text { concepteur pour l'analyse de comportement. }\end{array}$ & $\begin{array}{l}\text { Modélisé par un ensemble de } n \text { éléments } \\
\text { (déplacement, vitesse, état de } \\
\text { déformation, effort, inertie,...). } \\
\left.\qquad<\mathbf{M I}>=\mathbf{m i n}_{\mathbf{1}}, \mathbf{m i}_{\mathbf{2}}, \ldots, \mathbf{m i}_{\mathbf{n}}\right\}\end{array}$ \\
\hline $\begin{array}{c}\text { Hypothèses } \\
<\text { HYP> }\end{array}$ & $\begin{array}{l}\text { Ensemble des suppositions à adopter par le } \\
\text { concepteur afin de modéliser le produit dans } \\
\text { l'environnement où il évolue. Les hypothèses } \\
\text { portent sur la forme et la structure du produit, les } \\
\text { caractéristiques matérielles et les sollicitations } \\
\text { auxquelles sont soumises les différents } \\
\text { composants du produit. }\end{array}$ & $\begin{array}{l}\text { Modélisé par un ensemble de } m \\
\text { éléments portant sur la structure, la } \\
\text { forme, les sollicitations et les } \\
\text { caractéristiques matérielles du produit } \\
\left.\quad<\mathbf{H Y P}>=\text { hypp }_{\mathbf{1}}, \mathbf{h y p}_{\mathbf{2}}, \text {.., hyp } \mathbf{h}\right\}\end{array}$ \\
\hline $\begin{array}{c}\text { Modèle de Simulation } \\
\qquad<\mathrm{MS}>\end{array}$ & $\begin{array}{l}\text { Ensemble de formules, catalogues, procédures de } \\
\text { calcul,... qui sont susceptibles de donner une } \\
\text { évaluation des comportements mécaniques du } \\
\text { produit. Ces derniers dépendent directement des } \\
\text { domaines de calcul en question (cinématique, } \\
\text { dynamique, statique). }\end{array}$ & $\begin{array}{l}\text { Modélisé par un ensemble de } k \text { éléments } \\
\text { représentant les modèles de simulation } \\
\text { couramment utilisés pour donner une } \\
\text { évaluation du comportement mécanique } \\
\text { du produit } \\
\quad<\mathbf{M S}>=\left\{\mathbf{m s}_{\mathbf{1}}, \mathbf{m s}_{\mathbf{2}}, \ldots, \mathbf{m s}_{\mathbf{k}}\right\}\end{array}$ \\
\hline
\end{tabular}

Une feature de calcul particulière est un triplet composé d'un élément de l'ensemble $<$ MI $>$, d'un élément de l'ensemble $<$ HYP $>$ et d'un élément de l'ensemble $<\mathrm{MS}>$. Cela peut être formalisé sous la forme suivante.

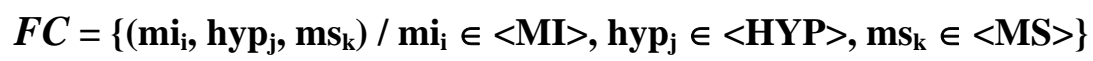

Dans nos études de cas, notre intérêt porte plus particulièrement sur les deux niveaux de modélisation technologique et technique. Dans le niveau technologique nous avons identifié les 
classes de calcul cinématique et statique. Dans le niveau technique nous avons identifié les classes de calcul poutre, plaque, coque et composant standard. Dans notre modèle, nous associons à chaque classe de problème de calcul une classe de feature de calcul. Dans ce chapitre nous allons nous intéresser aux features de calcul du niveau de définition des solutions technologiques.

\section{Classification des features de calcul :}

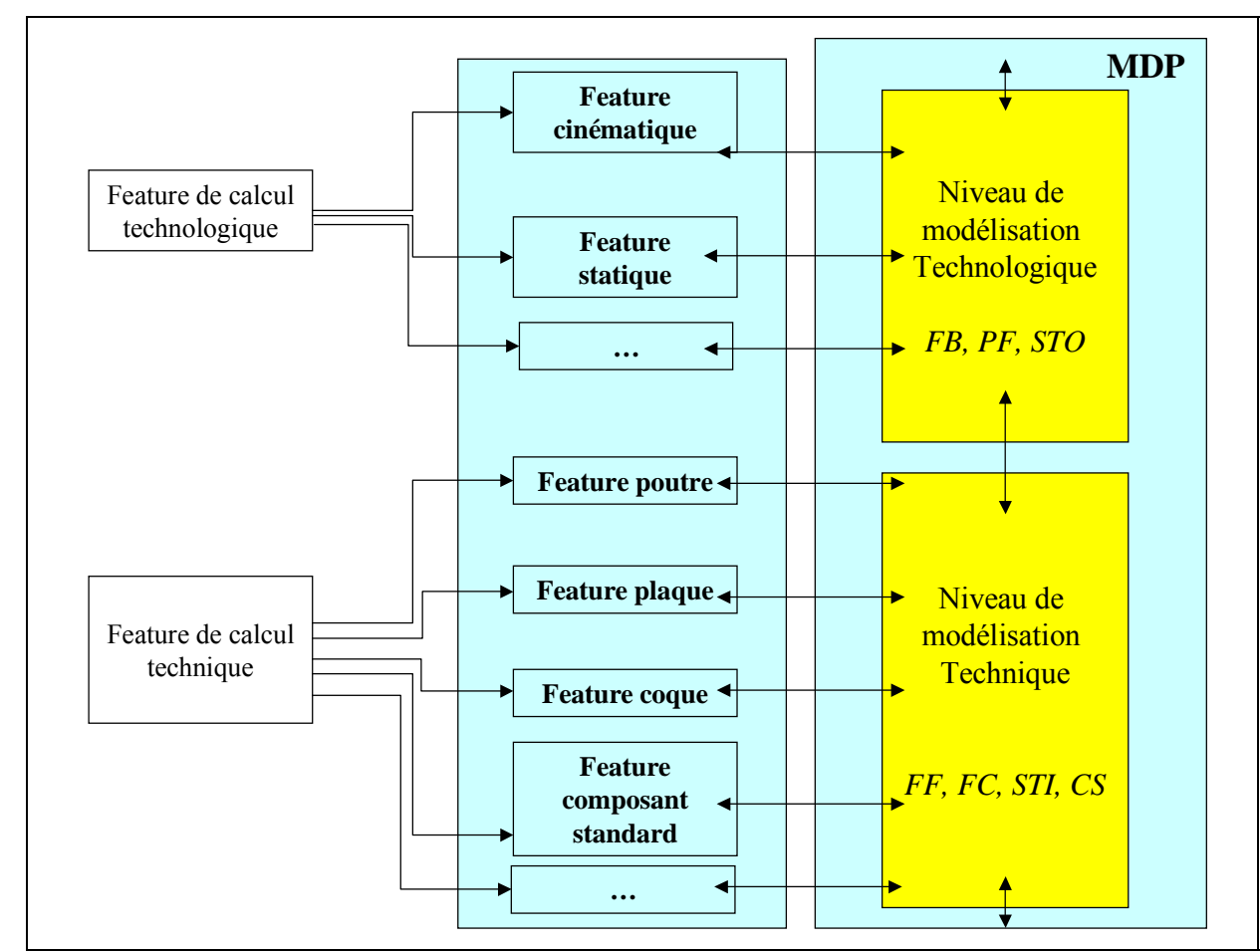

Classification des features de calcul

Une évaluation globale de l'approche d'intégration proposée par rapport à des critères d'évaluation objectifs révèle les caractéristiques suivantes :

- Capacité de la méthode à assurer la généricité en englobant de façon unique les différents concepts et modèles de calcul mécanique :

Sur les différentes tâches de calcul technologique et technique, nous avons montré que les features de calcul sont capables de s'appliquer au contexte considéré et de fournir au concepteur la démarche de modélisation du processus de calcul, mais aussi les données et les modèles nécessaires pour mener ces tâches de calcul.

- Capacité de la méthode à représenter de façon modulaire et interchangeable les différentes tâches de calcul :

Sur cet exemple nous avons illustré comment la feature statique a permis le calcul des efforts de liaisons et comment cette information a été récupérée par les features poutre et composant standard pour dimensionner les coussinets et les composants techniques de la solution technique du système de fermeture. Les features de calcul s'applique de façon modulaire pour répondre à un même problème de conception. Deux features de calcul appartenant à des niveaux de modélisation différents (en l'occurrence la feature statique du niveau de modélisation technologique et les features poutre et plaque du niveau de modélisation technique) peuvent collaborer ensemble pour résoudre un même problème de conception.

- Capacité de la méthode à assurer l'adaptabilité en ayant la faculté de s'instancier dans différents contextes :

L'exemple de conception du système de fermeture a montré qu'une même feature de 
calcul est capable de s'appliquer plusieurs fois dans un même contexte de conception avec des valeurs différentes. Elle peut également s'appliquer dans des contextes de conception similaires. La feature composant standard illustre cette propriété. En effet, elle a permis de dimensionner les différents coussinets du mécanisme (même contexte de conception et des valeurs différentes de l'effort et des dimensions). Cette même feature a permis aussi de dimensionner le vérin d'alimentation (instanciation dans un contexte différent).

- Capacité de la méthode à assurer la traçabilité, la modification et le recalcul :

La structure objet et les diagrammes de comportement des features de calcul garantissent la traçabilité des processus de calcul. Cela est illustré par la feature composant standard, la feature statique et la feature cinématique qui ont été instanciées plusieurs fois selon le même processus, avec des valeurs différentes pour résoudre des problèmes de conception différents. Des boucles de modifications sont également possibles dans les différents phases de modélisation du calcul, cela est assuré par la structure même de la feature de calcul.

Articles et Communications publiés :

- N. Aifaoui, A. BenAmara, A. Dogui, "Interopérabilité des processus de conception et de calcul »; Journal Européen de systèmes automatisés ; Vol 38-3-4 (2004) pp 443-464.

- B. Louhichi, N. Aifaoui, A. BenAmara, V. François, D. Deneux ; «Intégration numérique et interopérabilité des processus de CAO et de Calcul », XVII Congrès Français de Mécanique ; UTT -Troyes, France, 29 Août - 02 septembre 2005.

- N. Aifaoui, A. BenAmara ; «Les Features de calcul : Un modèle générique d'intégration $\mathrm{CAO} /$ Calcul» Premier Congrès International Conception et Modélisation des Systèmes Mécaniques : CMSM2005; 23-25 Mars 2005 ; Hammamet - Tunisie.

- N.Aifaoui, A. BenAmara, D. Deneux “ Towards a management model of design and mechanical analysis processes based on feature concept" ; $5^{\text {ème }}$ conférence internationale sur la conception et la fabrication intégrées en mécanique IDMME 04, Bath, UK avril 2004.

- N. Ifaoui, D. Deneux, A. BenAmara, R. Soenen, A. Dogui, "Mechanical analysis process modelling based on analysis features", IEEE international conference SMC' Hammamet, Tunisie, (6-9 oct 2002)

- N. Ifaoui, D. Deneux, A. BenAmara, R. Soenen, A. Dogui, "CAD/ Analysis integration based on generic model of mechanical analysis process", $4{ }^{\mathrm{e} m e}$ conference internationale sur la conception et la fabrication intégrées en mécanique: IDMME'02, IFMA, CLERMONTFERRAN, France, (14-16-mai 2002). 


\section{FICHE ENCADREMENT SCIENTIFIQUE}

\section{THESE DE DOCTORAT GENIE MECANIQUE}

Titre : « Intégration $\mathrm{CAO} / \mathrm{Calcul}$ par reconstruction du modèle $\mathrm{CAO}$ à partir des résultats éléments finis»

Financement / Formule : Codirection, Séjours alternés. Plein temps avec financement.

Période : Démarrage septembre 2003

Laboratoires Impliqués :

* Laboratoire de Génie Mécanique (Lab. Ma-05).

** Département de Génie Mécanique, Université du Québec à Trois Rivières.

Encadreurs : Abdelmajid BenAmara*

Vincent François**

Lotfi Romdhane*

Etudiant : $\quad$ Borhène Louhichi

\section{Présentation :}

La reconstruction du modèle CAO à partir des résultats de l'analyse Éléments Finis est devenu de plus en plus importante en conception intégrée, notamment pour la simulation en temps réel des processus de conception et/ou fabrication de produit mécanique (Simulation du processus d'emboutissage par exemple). En effet, lorsque l'on simule un comportement élasto-plastique d'une pièce dans un mécanisme ou bien un contact entre deux pièces, les déformations permanentes inhérentes à ces études entraînent une déformation du maillage qui peut devenir non valide ou de mauvaise qualité. La convergence de la solution numérique est alors bloquée parce que les données ne sont plus valides. Pour mener l'étude à son terme, le maillage doit être recalculé entre certaines étapes de la simulation. Autour des déformations permanentes, non seulement le maillage doit être recalculé mais les conditions aux limites doivent aussi être repositionnées sur la nouvelle forme du modèle. Dans un contexte de conception intégrée, les conditions aux limites sont saisies sur le modèle $\mathrm{CAO}$, c'est pourquoi les déformations du modèle éléments finis doivent être transmises au modèle CAO pour repositionner les conditions aux limites.

L'étude des problèmes d'assemblage et de simulation numérique du fonctionnement est actuellement abordée en considérant la configuration non déformée des pièces (solide rigide). $\mathrm{La}$ reconstruction des modèles $\mathrm{CAO}$ à partir des résultats de calcul tente de lever cette limitation et rend possible la visualisation et la simulation du comportement d'un assemblage à l'état déformé (état normal de fonctionnement) et détecter des éventuelles interférences. Ces dernières sont indétectables à l'état non déformé.

\section{Résultats :}

- L'étude des problèmes d'assemblage et de simulation numérique du fonctionnement des systèmes mécaniques est abordée par les systèmes de CAO en considérant la configuration non déformée des composants (solide rigide). La visualisation et la simulation du comportement de l'assemblage à l'état déformé (état normal de fonctionnement) est nécessaire puisque elle permet de détecter d'éventuelles interférences qui n'existent pas à l'état non déformé.

- La reconstruction dans le cas des changements géométriques du modèle CAO après Calcul éléments finis. 


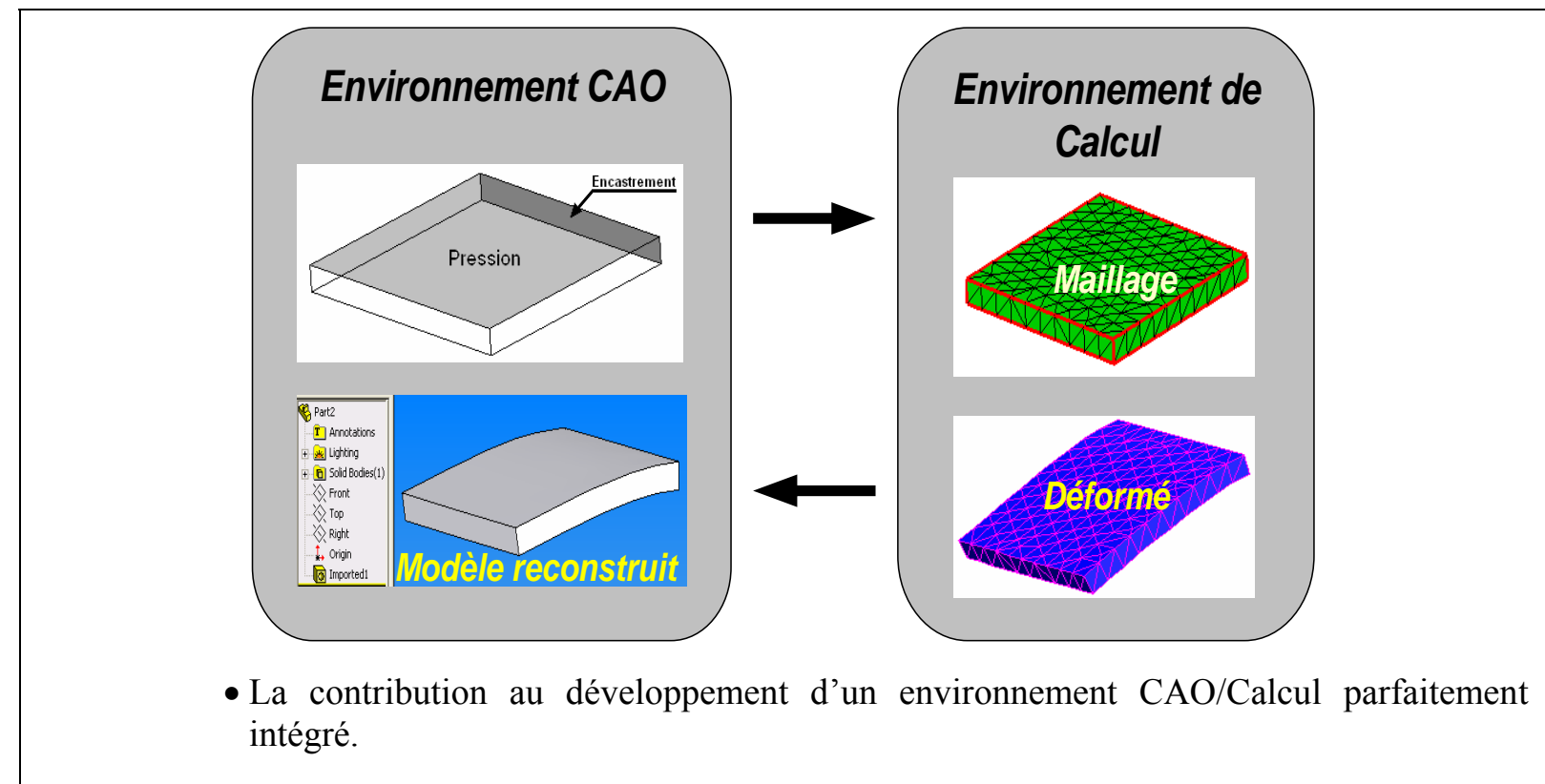

\section{Articles et Communications publiés :}

- B. Louhichi, A. Benamara, V. François ; Intégration CAO/Calcul par reconstruction des modèles CAO à partir des résultats de calcul ; Revue Internationale de l'Ingénièrie Numérique, 2005.

- B. Louhichi, A. Benamara, V. Francois ; Simulation d'un assemblage dans les conditions de fonctionnement ; 72e Congrès de l'ACFAS, Université du Québec à Montréal, 10 mai 2004.

- B. Louhichi, A. Benamara, V. Francois ; Reconstruction d'un modèle BREP à partir d'une analyse élémnts finis ; CMSM05, Hammamet, 23-25 mars 2005.

- B. Louhichi, V. Francois, A. Benamara; Intégration de la méthode des éléments finis dans le processus de CAO : détermination de la géométrie d'un modèle déformé ; 73e Congrès de l'ACFAS, Université du Québec à Chicoutimi, 12 mai 2005.

- B. Louhichi, A. Benamara, V. Francois, L. Romdhane; Rebuilding deformed CAD model starting from FEA results; Elastic linear case study; $20^{\mathrm{e}}$ Congrès Canadien de Mécanique Appliquée CANCAM 2005, Université de McGill, 30 mai au 2 juin 2005.

- B. Louhichi, N. Ifaoui, A. BenAmara, V. francois, D. Deneux; Intégration numérique et interopérabilité des processus de CAO et de Calcul ; Acceptée au $17^{\mathrm{e}}$ Congrès Français de Mécanique CFM 2005 ; Université de technologie de Troyes, 29 Août au 2 Septembre 2005.

- V. Francois, B. Louhichi, A. BenAmara ; Reconstruction d'un modèle CAO à partir d'un modèle éléments finis Déformé ; Acceptée au $17^{\mathrm{e}}$ Congrès Français de Mécanique CFM 2005 ; Université de technologie de Troyes, 29 Août au 2 Septembre 2005.

\section{Articles soumis :}

- A. BenAmara, N. Ifaoui, B. Louhichi, V. francois, D. Deneux; Intégration numérique et interopérabilité des processus de CAO et de Calcul ; Soumis au journal de Mécanique et Industries.

- A. BenAmara, V. François, B. Louhichi ; CAD/Analysis integration : Building CAD models using FEA deformed mesh; Soumis au Journal Computer Aided Design. 


\section{FICHE ENCADREMENT SCIENTIFIQUE}

\section{THESE DE GENIE MECANIQUE}

Titre : Caractérisation mécanique de l'usinage en finition

Financement / Formule : $\quad$ Sans financement/A temps partiel

Période : $\quad$ 2004-2007

Laboratoires Impliqués : $\quad$ Laboratoire de Génie Mécanique (Lab. Ma-05), ENIM.

\section{Co-encadreurs :}

\section{LOTFI ROMDHANE}

M. Abdelmajid BenAmara

Etudiant : $\quad$ Ramzi Fredj.

\section{Présentation :}

Les procédés d'usinage par enlèvement de copeaux occupent de plus en plus une place prédominante parmi les procédés de mise en forme des métaux.

Les avancés spectaculaires réalisés dans la construction des machines outils à commande numérique (rigidité, puissance, précision des déplacements et de commande et plages des vitesses.) couplées avec le développement des outils de coupe (formes, matériaux et revêtement) ont permis : d'augmenter le champs d'application de ces procédés comme l'usinage des aciers durcis et l'UGV; d'améliorer la précision dimensionnelle et la qualité des surfaces usinées.

Parmi les principales difficultés rencontrées, c'est de prédire dans des conditions de coupes imposées l'état de surface obtenue ainsi que son évolution au cours de l'usinage.

Objectif du travail: Prédiction de l'état de surface en usinage 3axes avec une fraise à bout sphérique.

Développement d'un modèle qui permet la prédiction de l'état de surface en intégrant principalement le phénomène de l'usure.

Les outils proposés pour l'étude sont : plan d'expérience, la régression multiple et les réseaux de neurones.

\section{Résultats :}

\section{Communications publiés :}

- R. Fredj, A. BenAmara; «Aperçu des techniques de prédiction de défauts des surfaces usinées » Premier Congrès International Conception et Modélisation des Systèmes Mécaniques : CMSM2005; 23-25 Mars 2005 ; Hammamet - Tunisie. 


\section{FICHE ENCADREMENT SCIENTIFIQUE}

\section{THESE DE DOCTORAT GENIE TEXTILE}

Titre : $\quad$ Contribution à l'élaboration d'algorithmes d'optimisation des problèmes de matelassage et de placement.

Financement/Formule : Sans financement / Temps partiel

Période : Démarrage septembre 2003.

Laboratoires Impliqués : *Laboratoire de Génie Mécanique, ENIM Monastir

**Unité de Recherches Textiles, ISET Ksar Helal.

Encadreurs : Abdelmajid BenAmara*, Faouzi Sakli**.

Etudiant : Wissem Guenanna**.

Présentation :

Ce travail s'inscrit dans le cadre d'une recherche universitaire susceptible d'intéresser l'industrie et a pour but final la réduction des pertes de la matière première (tissu) suite à l'opération de la coupe en utilisant des moyens totalement automatiques en un minimum de temps.

Il s'agit pratiquement d'élaborer des algorithmes contribuant à la résolution des problèmes de matelassage et de placement et ensuite les valider sur une maquette et qui va donner en premier lieu un plan de matelassage avec un minimum de matelas possible et en deuxième lieu déterminer pour chaque matelas les positions des pièces à couper qui donne le minimum de déchets.

- Le placement: Il s'agit de prendre tous les patrons des pièces constituants le vêtement pour les tailles du matelas considéré et ensuite les placer sur la couche supérieure du matelas qui est souvent de forme rectangulaire, dont la largeur dépend de la largeur du rouleau de tissu et la longueur est variable). L'opérateur essaye de minimiser au maximum les inter-espaces entre les patrons placés (perte matière) et en respectant plusieurs contraintes.

- Le matelassage: Il s'agit d'imbriquer le maximum de tailles sur un seul matelas pour gagner en terme d'efficience et minimiser la perte matière et de minimiser le nombre de matelas pour gagner en terme de temps et d'argent. Ce plan de matelassage est réalisé par des méthodes manuelles basées sur le tâtonnement et le savoir-faire (ex: méthode de glissement) qui prennent souvent beaucoup de temps et n'aboutissent pas à des résultats satisfaisants.

\section{Résultats :}

Une large Etude Bibliographique a été réalisé et une méthode d'optimisation des plans de matelassage basée sur les algorithmes génétiques a été proposée. Le travail sur la partie placement n'a pas été encore abordée de façon profonde et bute encore sur des difficultés de formulation et de mise en œuvre informatique.

\section{Communications publiées :}

W. Guenenna, A. BenAmara, F. Sakli, « Optimisation des plans de matelassage à l'aide des algorithmes génétiques » The First International Conference of Applied Research in Textile ; CIRAT ; pages 143-150, Monastir (2004). 
Fiches Encadrement Mastère 


\section{FICHE ENCADREMENT SCIENTIFIQUE}

\section{MASTERE DE GENIE MECANIQUE}

Titre : Développement d'un solveur élastoplastique à l'aval d'un mailleur tridimensionnel préoptimisé intégré à la $\mathrm{CAO}$

\begin{tabular}{ll}
\hline Financement / Formule : & Plein temps, sans financement \\
\hline Soutenu le : & $25 / 05 / 2005$
\end{tabular}

Laboratoires Impliqués :

* Laboratoire de Génie Mécanique (Lab. Ma-05).

** Département de Génie Mécanique, Université du Québec à Trois Rivières.

\section{Co-encadreur : $\quad$ BENAMARA ABDELMAJID*}

BELHADJSALAH DEDI* FRANCOIS VINCENT**

\section{Présentation :}

L'intégration de Calcul dans les systèmes CFAO, constitue un axe de recherche d'actualité. L'un des principaux objectifs de cette thématique est de rapprocher aux mieux les deux environnements CAO et CALCUL, dans le souci d'améliorer et de rendre plus efficace l'interaction et la communication entre ces deux mondes. Dans ce cadre, ce travail consiste à mettre en place un solveur élastoplastique résolvant les problèmes des structures en petites déformations complètement intégré à la CAO à l'aval du mailleur intégré à la CAO MAGIC.

Le mailleur MAGIC permet la saisie des conditions aux limites à partir des entités topologiques (modèle CAO) et gardent des liens de dépendances entre le maillage et le modèle CAO. Cependant, le solveur proposé permet de se baser sur la structure de données de ce mailleur permet d'effectuer des calcul de structure élastoplastiques en se basant sur un algorithme développé dans notre laboratoire LGM. Cet algorithme permet d'effectuer des calculs dans le domaine plastique en se basant sur un calcul purement élastique. Cela permet d'écarter le calcul des matrices tangentes et leur inversion, qui est très coûteux de points de vue implémentations et calcul et principalement les problèmes de convergences liées aux algorithmes itératifs.

\section{Résultats :}

Dans ce travail de mastère, on a pu mettre en place un solveur permettant de résoudre à la fois un problème de calcul de structure élastique et élastoplastique.

Confrontés avec des codes de calcul commerciaux (ABAQUS6.4, CosMosM, DsignStar) les résultats que nous avons obtenus sont satisfaisants.

Dans notre démarche de validation, nous sommes parti des cas simples en géométrie et en conditions aux limites (cube, essai de traction, cisaillement homogène et quelconque), puis nous avons poussé notre validation vers des cas plus complexes au niveau de la géométrie aussi bien qu'au niveau des conditions aux limites (calcul d'une bielle).

Les résultas du calcul élastique et élastoplastique (déplacements, déformations, contraintes, déformations plastiques, contraintes équivalentes de Von Mises) sont stockés dans une structure de données accessible par l'utilisateur en mode programmé ou en format fichier texte. La première est en format texte. Ces grandeurs peuvent être aussi visualisées sous forme d'isovaleurs. 


\section{FICHE ENCADREMENT SCIENTIFIQUE}

\section{MASTERE DE GENIE MECANIQUE}

Titre :

Etude expérimentale et numérique du tournage dur

Financement / Formule : Sans financement, à temps partiel

Soutenu le : $\quad 13 / 05 / 2005$

Laboratoires Impliqués : LGM

Etudiant : Imed Hajjaji

Collaborateurs : $\quad$ Ramzi Fredj, LGM ENIM.

\section{Présentation :}

Ce travail est consacré à l'étude expérimentale et numérique du tournage dur.

L'étude expérimentale concerne l'usinage de l'acier X155CrMoV12 avec un outil en CBN. Sa finalité est l'analyse du phénomène d'usure des outils de coupe en tournage dur, l'étude de la qualité de la surface usinée ainsi que l'évolution des efforts de coupe en fonction des paramètres d'usinage (la l'avance, la vitesse de coupe et la profondeur de passe). L'étude et la détermination des facteurs influents sont également parmi les objectifs de ce travail.

Une étude numérique est développée à l'aide des codes de calcul Abaqus. L'objectif de cette étude est d'analyser le comportement cinématique de l'outil de coupe sous l'effet des efforts de coupe ainsi que les paramètres d'influence.

\section{Résultats :}

Cette étude expérimentale et numérique nous a permis de conclure que :

- La rugosité de la surface est affectée par l'avance et en deuxième lieu par la vitesse de coupe. La profondeur de passe n'a pas d'effet considérable sur la dégradation de l'état de la surface.

- La composante radiale de l'effort de coupe est considérée comme l'effort principal en tournage dur. C'est cette composante qui est la plus sensible à la variation des paramètres de coupe et à l'usure.

- Les mécanismes de l'usure les plus dominants en tournage dur sont l'usure par abrasion et l'usure par adhésion, ce qui est conforme avec la bibliographie.

- Les modèles prédictifs exponentiels obtenus, de l'évolution des efforts et de la rugosité de la surface en fonction des paramètres d'usinage présentent relativement une bonne précision.

- La cinématique de la pointe de l'outil a une influence sur la qualité de la surface. C'est l'effet de la rigidité du système usinant. Un choix judicieux des paramètres de coupe permet de minimiser l'amplitude du déplacement de la pointe de l'outil.

\section{Communications publiés :}

« Etude expérimentale de l'influence des paramètres de coupe sur l'état de surface en tournage dur » CMSM2005, Hammamet 23-25 mars 2005 


\section{FICHE ENCADREMENT SCIENTIFIQUE}

\section{MASTERE DE GENIE MECANIQUE}

Titre : Simulation numérique de l'emboutissage des tôles minces

Financement / Formule : $\quad$ Sans financement/A temps partiel

Soutenu le : $\quad 05 / 04 / 2003$

Laboratoires Impliqués : $\quad$ Laboratoire de Génie Mécanique (Lab. Ma-05), ENIM.

Etudiant : $\quad$ Abdessalem Chameckh

Co-encadreurs : $\quad$ Abdelmajid BenAmara; Hedi BelHadjSalah

\section{Présentation :}

L'objectif de ce travail était d'analyser numériquement l'emboutissabilité des tôles minces. Les résultats obtenus sont comparés avec des résultats expérimentaux. En utilisant le code de calcul par éléments finis ABAQUS, et par suite le modèle de comportement élastoplastique anisotrope formulé en grandes déformations intégré dans ABAQUS, nous avons simulé l'essai de gonflement hydraulique et l'essai d'emboutissage en retreint (essai similaire à l'essai de Swift).

Nous avons commencé par la simulation de l'essai de gonflement hydraulique et nous avons pu constater l'apparition d'un écart entre les résultats numériques et expérimentaux lors de confrontations de deux réponses (variation de la pression en fonction de la hauteur au pôle). Cette différence peut être expliquée par le fait que la loi constitutive implémentée dans ABAQUS ne reflète pas correctement le comportement de la tôle ou d'une "pauvre" identification des constantes du modèle dû à l'insuffisance des résultats expérimentaux. Nous pensons, aussi, que le couplage plasticité anisotrope endommagement peut conduire à une solution plus réaliste.

\section{Résultats :}

L'étude de l'influence des différents paramètres intervenant dans la réponse numérique de la tôle nous a permis de montrer que :

- L'écart entre ces résultats, expérimentaux et numériques, ne vient pas d'une erreur de discrétisation (nombre de couche dans l'épaisseur de la tôle).

- Cet écart ne vient pas non plus des choix des conditions aux limites considérées.

- L'écart est très sensible à la manière d'identifier la courbe d'écrouissage (soit qu'on l'identifie à partir de l'essai de traction simple ou de l'essai d'expansion équibiaxiale).

Pour caler les deux réponses expérimentale et numérique, nous avons ajuster la courbe d'écrouissage en utilisant celle obtenue par l'essai de traction simple et celle identifiée à partir de l'essai d'expansion équibiaxiale (figure 9).

En conclusion, nous pensons que le modèle d'écrouissage isotrope ne suffit pas pour décrire le comportement du matériau utilisé. En effet, il apparaît à travers des simulations effectuées que la surface de plasticité n'évolue pas de manière homothétique.

\section{Articles publiés :}

A. Chamekh, H. BelHadjSalah, M. A. Gahbiche, A. BenAmara, A. Dogui, "Identification of the hardening curve using a finite element simulation of the bulge test" Steel GRIPS Journal of steel and related materials; Septembre 2004 741-746 (2004) 


\section{FICHE ENCADREMENT SCIENTIFIQUE}

\section{MASTERE GENIE MECANIQUE}

Titre : «Reconstruction d'un modèle BREP à partir des résultats éléments finis : cas de l'élasticité linéaire»

Financement / Formule : Codirection, Séjours alternés. Plein temps avec financement.

Soutenu le : $07 / 07 / 2003$.

Laboratoires Impliqués :

* Laboratoire de Génie Mécanique (Lab. Ma-05).

** Département de Génie Mécanique, Université du Québec à Trois Rivières.

Encadreurs : Abdelmajid BenAmara*

Vincent François**

Etudiant : $\quad$ Borhène Louhichi

\section{Présentation :}

Ce travail de mastère rentre dans le cadre de l'intégration $\mathrm{CAO} / \mathrm{Calcul}$ et plus précisément l'intégration de la méthode des éléments finis dans le processus $\mathrm{CAO} / \mathrm{FAO}$, dans le but de la définition d'un modèle Élément finis/CAO unifié : cette définition nécessite la mise à jour de CAO modèle quand le modèle de calcul est modifié. En d'autre terme le retour du modèle géométrique déformé de l'environnement de calcul vers celui de la CAO.

La complexité du problème de reconstruction nécessite sa division en plusieurs étapes complémentaires :

La reconstruction dans le cas de petites déformations : La nature des entités du modèle CAO est la même avant et après déformations (Topologie et géométrie). Seulement les paramètres sont modifiés (point d'insertion, vecteur directeur...)

La reconstruction dans le cas des grandes déformations : La géométrie et/ou la géométrie et la topologie du modèle CAO sont modifiés

L'objectif de ce travail de mastère et de montrer la faisabilité du projet au complet et initier le développement d'un outil de reconstruction à travers la résolution du premier point mentionné précédemment.

\section{Résultats :}

Les résultats de ce mastère présentent un apport majeur notamment dans le cas suivant :

Actuellement dans les environnements CAO lorsqu'on simule le fonctionnement d'un assemblage, on ne peut pas détecter les interférences et les collisions qui existent entre les différents composants du système, puisque les déformations qui existent en réalité entre les différents pièces de système ne sont pas pris en compte dans le modèle CAO. La reconstruction des modèles CAO à partir des résultats de calcul tente de lever cette limitation et rend possible la visualisation et la simulation du comportement d'un assemblage à l'état déformé (état normal de fonctionnement) et détecter des éventuelles interférences. Donc le développement de cet outil constitue un enrichissement pour les systèmes $\mathrm{CAO}$. 


\section{FICHE ENCADREMENT SCIENTIFIQUE}

\section{MASTERE DE GENIE MECANIQUE}

Titre :

Etude expérimentale et numérique du tournage dur

Financement / Formule : Sans financement

Soutenu le : $\quad 02 / 03 / 2002$

Laboratoires Impliqués : LGM

Etudiant : Fathi Bouabda.

\section{Présentation :}

Notre travail intitulé « Modèles de Conception Basés sur les Entités Technologiques, Application à la conception des moules d'injection plastiques», rentre dans ce cadre, puisqu'il tente de mettre en œuvre un modèle permettant de concevoir des plaques empreintes de moules d'injection plastique en tenant compte dès le départ des contraintes de fabrication, au même titre que des contraintes fonctionnelles. Ceci sera possible en concevant dès le départ, par des opérations topologiques et des entités qui tiennent compte des opérations de fabrication et des capacités et moyens qui sont disponibles au sein de l'entreprise.

En effet, les opérations d'enlèvement de la matière sont représentées par des opérations topologiques de soustraction, les formes sont insérées par des entités réalisées par un outil ou un procédé de fabrication donnés. Ce qui revient à proposer un modèle capable d'insérer, en plus de celles à caractère géométrique, des informations technologiques d'origines diverses. Ce qui explique bien la richesse du modèle de produit et la diversité de la nature des données qu'il est sensé contenir.

\section{Résultats :}

Ce travail à été basé sur le formalisme OMT pour présenter notre modèle. Nous avons expliqué les étapes de la construction d'un modèle de moule par l'intermédiaire de notre propre modèle de conception des moule MoFMo.

Nous avons également montré les avantages de notre modèle confrentés aux fontionnalité des modeleurs solides paramétriques usuels tels que ProEngeneer ${ }^{\circledR}$ et SolidWorks ${ }^{\circledR}$. Ces avantages résident essentiellement dans l'identification d'une part des différents composants d'un moule (dans une perception d'ensemble) et d'autre part des différentes formes au sein d'un composant du moule (dans une perception de détail). En effet, l'identification de n'importe quelle feature sur un composant du moule, nous permet d'accéder à une mine d'informations. Ces informations peuvent être utilisées soit pour créer soit pour modifier d'autres instances de classes provenant de n'importe quelle personne de n'importe quelle application. Cela favorise l'adoption de le l'ingénierie concourante, comme mode d'organisation du processus de conception, permettant ainsi une parfaite intégration entre deux ou plusieurs activités

\section{Articles et Communications publiés :}

«Modèle de conception basé sur les entités technologiques » Le Premier Colloque de Recherche Appliquée et de Transfert de Technologie, Radès, Tunisie 11-12 décembre 2002. 


\section{ANNEXE III :}

Fiches Projet de Collaboration Scientifique 


\section{COOPERATION DGRST-CNRS ACTIONS D'ECHANGES}

TITRE DU PROJET

Modélisation de vêtements par features d'assemblages intégrant les lois de comportement des matériaux souples. Contribution à la confection assistée par ordinateur

Discipline : Sciences et Techniques de I'Ingénieur

Mots clés : Modélisation, Confection Assistée par ordinateur, Modélisation features.

Code $: . .03 /$ R 1108........ Date du début du projet (le cas échéant) : .....2003.

\section{RESPONSABLES DU PROJET}

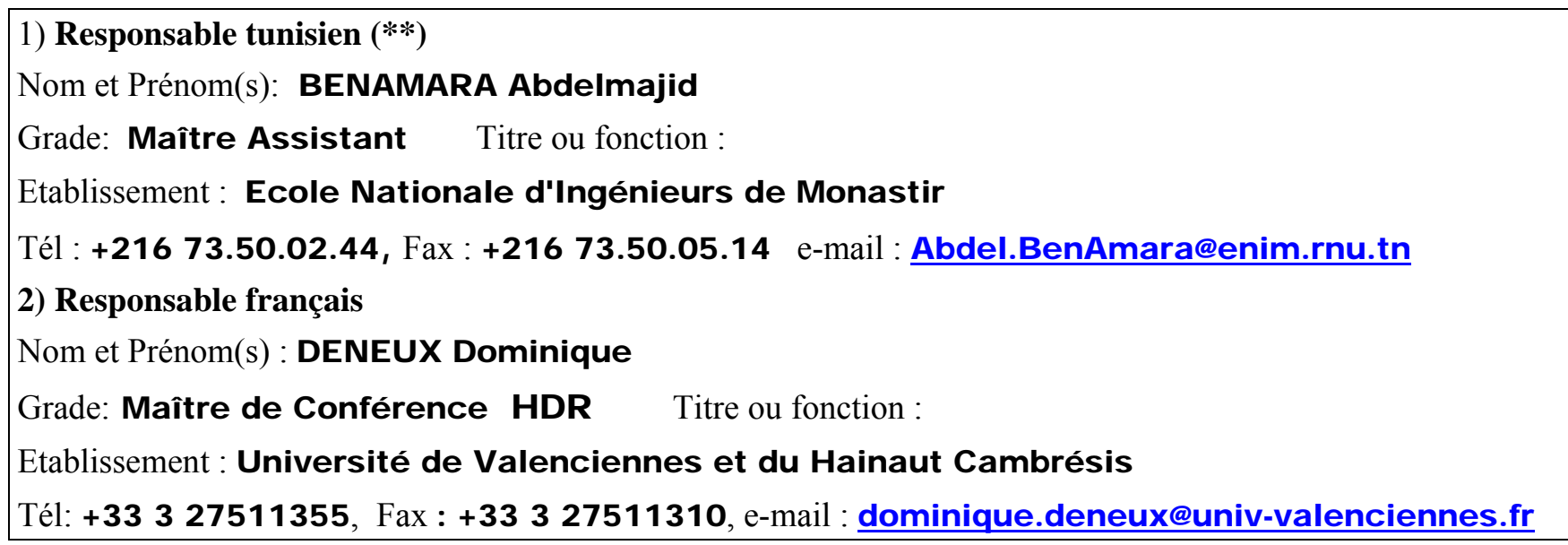

\section{CHERCHEURS ENGAGES DANS L'ACTION D'ECHANGES}

1) Chercheurs en Tunisie

\begin{tabular}{|l|l|l|l|l|l|l|}
\hline \multicolumn{1}{|c|}{$\begin{array}{c}\text { Nom(s) \& } \\
\text { Prénom(s) }\end{array}$} & $\begin{array}{c}\text { Grade ou } \\
\text { qualité }\end{array}$ & $\begin{array}{l}\text { Etablisse } \\
- \text { ment(s) }\end{array}$ & \multicolumn{4}{|c|}{ Objectifs des Activités de Recherche } \\
\hline & & & Encadrement & $\begin{array}{l}\text { Act. Post. } \\
\text { Doctorale }\end{array}$ & $\begin{array}{l}\text { Act. Rech. } \\
\text { Diplomantes }\left({ }^{*}\right)\end{array}$ & Habilitation (Hb) \\
\hline ROMDHANE Lotfi & $\begin{array}{l}\text { Maître de } \\
\text { conférence }\end{array}$ & ENIM & $\bullet$ & & & \\
\hline $\begin{array}{l}\text { BENAMARA } \\
\text { Abdelmajid }\end{array}$ & $\begin{array}{l}\text { Maître } \\
\text { Assistant }\end{array}$ & ENIM & $\bullet$ & & & - \\
\hline ELAMRI Adel ${ }^{*}$ & Thésard & ENIM & & & $\begin{array}{c}\text { ・ } \\
\text { Thèse en } \\
\text { cotutelle }\end{array}$ & \\
\hline
\end{tabular}




\section{2) Chercheurs en France}

\begin{tabular}{|l|l|l|l|l|l|l|}
\hline \multicolumn{1}{|c|}{$\begin{array}{c}\text { Nom(s) \& } \\
\text { Prénom(s) }\end{array}$} & $\begin{array}{c}\text { Grade ou } \\
\text { qualité }\end{array}$ & $\begin{array}{l}\text { Etablisse } \\
- \text { ment(s) }\end{array}$ & \multicolumn{5}{|c|}{ Objectifs des Activités de Recherche } \\
\hline & & & $\begin{array}{l}\text { Encadreme } \\
n t\end{array}$ & $\begin{array}{l}\text { Act. Post. } \\
\text { Doctorale }\end{array}$ & $\begin{array}{l}\text { Act. Rech. } \\
\text { Diplomantes (*) }\end{array}$ & $\begin{array}{l}\text { Habilitation } \\
(\mathrm{Hb})\end{array}$ \\
\hline $\begin{array}{l}\text { DENEUX } \\
\text { Dominique }\end{array}$ & $\begin{array}{l}\text { Maître de } \\
\text { conférence } \\
\text { HDR }\end{array}$ & LAMIH & $\bullet$ & & & \\
\hline BONTE Thérèse & $\begin{array}{l}\text { Ingénieur de } \\
\text { recherche }\end{array}$ & LAMIH & - & & & \\
\hline ELAMRI Adel * & Thésard & ENIM & & & $\begin{array}{l}\text { lò̀se en } \\
\text { cotutelle }\end{array}$ & \\
\hline
\end{tabular}

MOYENS ACCORDES POUR 2001

1) Missions de chercheurs français en Tunisie

\begin{tabular}{|c|l|l|c|}
\hline Nom(s) et Prénom(s) & \multicolumn{1}{|c|}{ Grade } & \multicolumn{1}{c|}{ Objet du séjour } & Durée \\
\hline BONTE Thérèse & $\begin{array}{l}\text { Ingénieur de } \\
\text { Recherche }\end{array}$ & $\begin{array}{l}\text { Suivi de la thèse de M. Guenanna } \\
\text { séminaire }\end{array}$ & 10jours \\
\hline
\end{tabular}

2) Séjours de chercheurs tunisiens en France

\begin{tabular}{|c|l|l|c|}
\hline Nom(s) et Prénom(s) & \multicolumn{1}{|c|}{ Grade } & \multicolumn{1}{c|}{ Objet du séjour } & Durée \\
\hline BenAmara Abdelmajid & Maître Assistant & $\begin{array}{l}\text { Séminaire } \\
\text { Publications scientifiques }\end{array}$ & 1 mois \\
\hline
\end{tabular}

\section{PRESENTATION DU PROJET}

$\mathrm{Au}$ cours de la dernière décennie, l'industrie de l'habillement a connu un accroissement de la concurrence au niveau mondial, accompagné d'une forte augmentation des exigences de productivité et de réactivité par rapport aux attentes fluctuantes du marché. Le processus de fabrication des vêtements a été amélioré grâce à l'introduction d'outils informatiques, favorisant ainsi la réduction des coûts et des délais de fabrication. Cependant, la conception des vêtements est toujours effectuée, en grande partie, de manière empirique et manuelle. Il s'agit essentiellement d'un processus créatif et artistique.

Un système de conception destiné aux concepteurs de vêtements ne doit pas être source de restriction de la créativité ou contraignant vis à vis de la dimension artistique de leur activité. Les concepteurs de vêtements (stylistes et modélistes), opèrent la conception en agissant sur de nombreux paramètres métiers, en mobilisant leur expertise; ils évaluent, en général après réalisation d'un prototype physique, leurs propositions en termes d'esthétique, de fonctionnalité, de " fabricabilité », et de coût de revient. L'une des principales difficultés rencontrées lors des tentatives de développement d'outils informatiques support de la conception des vêtements est liée à la complexité de représentation du comportement des matériaux souples et à la représentation simulée de la forme du vêtement quand il sera porté.

Les systèmes commerciaux positionnés sur ce créneau sont peu nombreux et limités à quelques suites logicielles comprenant des bases de données relatives aux tissus et aux styles, ou des outils graphiques 2D permettant la création des motifs colorés des tissus. Au niveau du patronage, plusieurs outils de gradation et de placement existent sur le marché. Ils permettent la transformation des patrons de base en des patrons dans les différentes tailles, par l'application des lois d'évolution. Mais, pour la plupart des étapes de la conception, aucune aide n'existe. Un grand nombre de problèmes ne sont donc pas résolus, en particulier.

Une insuffisance du système support de l'activité du modéliste, qui ne dispose pas d'outil efficace pour concevoir les patrons et obtenir une représentation 3D du vêtement porté. 
L'absence d'expertise métier dans le système: l'intuition empirique prédomine. Le modéliste se comporte plus en artiste qu'en technicien.

L'inadéquation du modèle du vêtement manipulé : le modèle du vêtement ne s'exprime que de manière physique (papier pour le croquis de style, toile pour les patrons et les prototypes) tout au long du cycle de sa conception. Les systèmes actuels ne disposent pas de modèle informatique intégrant toutes les caractéristiques du vêtement.

Sur le plan de la recherche, plusieurs travaux dont /Curchesne, 1995/, /McCartney, 1999/ et /Bonte, 2000/ ont abouti au développement d'outils ponctuels de conception et de simulation de pièces en matériaux souples. Deux approches de la confection assistée par ordinateur sont envisageables. La première, qui concerne l'essentiel des travaux, est basée sur la démarche suivante : Conception de patrons 2D sur un logiciel dédié, positionnement des patrons sur un mannequin numérique, transformation des patrons $2 \mathrm{D}$ en pièces $3 \mathrm{D}$, assemblage des composants du vêtement, application du modèle de déformation. La seconde s'opère en 3D et permet au concepteur de concrétiser directement ses intentions créatrices, avant de vérifier la fabricabilité de chaque composant en 2D. Cette approche a été suivie par quelques groupes de recherche dont /Hinds, 1990/ et /McCartney, 1999/, /Cugini, 2002/ et le LAMIH /Bonte, 2000/. Néanmoins, ces approches restent des pistes de recherche non intégrées dans de réels systèmes de conception. Il manque encore un lien entre les outils mathématiques et les outils dont le modéliste a réellement besoin. Ceci est dû en partie à l'absence de bibliothèque de fonctions " métier » exploitable par les concepteurs textiles, et à la difficulté de créer des liens de cause à effet entre les fonctions du produit et les formes physiques.

L'approche de conception par features d'assemblage semble pouvoir apporter quelques avantages dans le développement d'un outil de confection assistée par ordinateur. /Bronsvoort, 1993/ définit les features comme des formes génériques résultant d'opérations sur les formes facilement utilisables par le concepteur pour modéliser un objet. Pour /Shah, 1992/, les features d'assemblage sont des associations entre deux features de forme, sur des pièces en interaction. Le projet CEE-FEAST /Deneux, 1999/, auquel le LAMIH a participé, a permis d'identifier un catalogue de features d'assemblage utilisables dans le processus de définition routinière des composants d'un avion, allant de la caractérisation d'un problème de conception (attachement ou interférence) jusqu'à la définition détaillée de solutions génériques contextualisées.

Dans le projet présenté, nous chercherons à projeter l'approche validée dans FEAST sur le processus de confection. Le vêtement sera considéré comme un ensemble de pièces (patrons) assemblées par différents types de liaisons (coutures, boutons, ...) et soumis aux lois de comportement propres aux matériaux souples. Le modèle produit du vêtement basé sur des features d'assemblage et des features de pièces de tissus permettra de supporter le processus de confection, en fournissant au modéliste un haut niveau d'interaction avec le modèle de vêtement.

La collaboration sera jalonnée de deux diplômes universitaires incluant une thèse en cotutelle et une habilitation à diriger des recherches. 


\section{COOPERATION DGRST-CNRS ACTIONS D'ECHANGES}

TITRE DU PROJET

Modèles innovants en conception mécanique

Discipline : Sciences et Techniques de l'Ingénieur

Mots clés : Conception, Innovation, CAO, Calcul, Features, Intégration.

Code $: .$. code 01/R 1103........ Date du début du projet (le cas échéant) : .....2001

\section{I- RESPONSABLES DU PROJET}

1) Responsable tunisien $(* *)$

Nom et Prénom(s): BENAMARA Abdelmajid

Grade: Maître Assistant Titre ou fonction : Directeur du Département de G.M.

Etablissement: Ecole Nationale d'Ingénieurs de Monastir

Téléphone : 03500244 Télécopie : 03500514 e-mail : Abdel.BenAmara@enim.rnu.tn

2) Responsable français

Nom et Prénom(s) : SOENEN René

Grade: Professeur Titre ou fonction : Directeur de recherche au LAMIH

Etablissement : Université de Valenciennes et du Hainaut Cambrésis

Téléphone: (33)327511325 Télécopie : (33) 327511310 e-mail : Soenen@univ-valenciennes.fr

\section{II- CHERCHEURS ENGAGES DANS L'ACTION D'ECHANGES}

1) Chercheurs en Tunisie

\begin{tabular}{|c|c|c|c|c|c|c|}
\hline \multirow[t]{2}{*}{$\begin{array}{l}\operatorname{Nom}(s) \& \\
\text { Prénom(s) }\end{array}$} & \multirow[t]{2}{*}{$\begin{array}{l}\text { Grade ou } \\
\text { qualité }\end{array}$} & \multirow[t]{2}{*}{$\begin{array}{l}\text { Etablisse } \\
\text { - ment(s) }\end{array}$} & \multicolumn{4}{|c|}{ Objectifs des Activités de Recherche } \\
\hline & & & Encadrement & $\begin{array}{l}\text { Act. Post. } \\
\text { Doctorale }\end{array}$ & $\begin{array}{c}\text { Act. Rech. } \\
\text { Diplomantes }(*)\end{array}$ & $\begin{array}{l}\text { Habilitation } \\
(\mathrm{Hb})\end{array}$ \\
\hline $\begin{array}{l}\text { DOGUI } \\
\text { Abdelwaheb }\end{array}$ & Professeur & ENIM & $\bullet$ & & & \\
\hline $\begin{array}{l}\text { BENAMARA } \\
\text { Abdelmajid }\end{array}$ & $\begin{array}{c}\text { Maître } \\
\text { Assistant }\end{array}$ & ENIM & 0 & & & $\bullet$ \\
\hline IFAOUI Nizar * & Thésard & ENIM & & & Thèse en cotutelle & \\
\hline
\end{tabular}




\section{2) Chercheurs en France}

\begin{tabular}{|c|c|c|c|c|c|c|}
\hline $\begin{array}{l}\text { Nom(s) \& } \\
\text { Prénom(s) }\end{array}$ & $\begin{array}{c}\text { Grade ou } \\
\text { qualité }\end{array}$ & $\begin{array}{l}\text { Etablisse } \\
\text { - ment(s) }\end{array}$ & \multicolumn{4}{|c|}{ Objectifs des Activités de Recherche } \\
\hline & & & Encadrement & \begin{tabular}{|l} 
Act. Post. \\
Doctorale
\end{tabular} & $\begin{array}{l}\text { Act. Rech. } \\
\text { Diplomantes (*) }\end{array}$ & Habilitation $(\mathrm{Hb})$ \\
\hline SOENEN René & Professeur & LAMIH & $\bullet$ & & & \\
\hline $\begin{array}{l}\text { DENEUX } \\
\text { Dominique }\end{array}$ & $\begin{array}{l}\text { Maître de } \\
\text { conférence }\end{array}$ & LAMIH & 0 & & & $\bullet$ \\
\hline IFAOUI Nizar * & Thésard & ENIM & & & $\begin{array}{l}\text { Thèse en } \\
\text { cotutelle }\end{array}$ & \\
\hline
\end{tabular}

MOYENS ACCORDES POUR 2001

1) Missions de chercheurs français en Tunisie

\begin{tabular}{|c|c|l|c|}
\hline Nom(s) et Prénom(s) & \multicolumn{1}{|c|}{ Grade } & \multicolumn{1}{c|}{ Objet du séjour } & Durée \\
\hline Deneux Dominique & Maître de conférence & $\begin{array}{l}\text { Suivi de la thèse deM. IFAOUI - } \\
\text { publications scientifiques + } \\
\text { séminaire }\end{array}$ & 10jours \\
\hline
\end{tabular}

2) Séjours de chercheurs tunisiens en France

2) Séjours de chercheurs tunisiens en France
\begin{tabular}{|c|c|l|c|}
\hline Nom(s) et Prénom(s) & Grade & \multicolumn{1}{c|}{ Objet du séjour } & Durée \\
\hline BenAmara Abdelmajid & Maître Assistant & $\begin{array}{l}\text { Suivi de la thèse deM. IFAOUI - } \\
\text { publications scientifiques + } \\
\text { séminaire }\end{array}$ & 1 mois \\
\hline
\end{tabular}

\section{PRESENTATION DU PROJET}

Pour être compétitives, les entreprises grandes ou petites ont besoin de développer les partenariats internes et externes, et l'activité de conception de produits et systèmes industriels s'opère aujourd'hui nécessairement dans un contexte collaboratif [HAT-96]. Les outils de CAO les plus récents, censés supporter cette activité, ne proposent pourtant que des solutions isolées à l'usage des métiers, ou bien des outils généraux limités aux aspects géométriques de la conception. L'intégration des différents métiers de la conception et de leurs méthodes spécifiques requiert la définition de modèles de produits sémantiquement plus riches, porteurs d'informations métiers [KRA-93][DEN-99]. Dans le cas particulier du concepteur mécanicien, les outils de CAO actuels confinent son rôle à l'analyse de solutions élaborées préalablement par d'autres acteurs, cristallisant ainsi une approche séquentielle. La recherche de méthodes et modèles pour la conception est donc un thème plus que jamais d'actualité, et par nature pluridisciplinaire.

Dans le cadre du présent projet, nous souhaitons aborder ce thème dans le cadre de la conception mécanique, en associant l'expérience du LAMIH en formalisation de l'activité de conception et en modélisation de produit et système suivant une démarche systémique [JAC-98][GRU-99], à l'expertise [ROM-96] du Laboratoire de Génie Mécanique de l'ENIM reconnu par la DGRST. Depuis 1994, une collaboration soutenue entre les deux laboratoires a déjà donné lieu à plusieurs publications scientifiques (liste en annexe). Une thèse en cotutelle, celle de M. BENAMARA [BEN-98] (résumé en annexe), a été soutenue en 1998. Ces travaux communs se poursuivent actuellement avec la thèse en cotutelle de M. N. IFAOUI, portant sur la définition d'un modèle de caractéristiques de calcul (sujet en annexe).

Le thème support de notre collaboration pour l'année 2000-20001 coïncide avec le sujet de thèse de $\mathrm{M}$. N. IFAOUI. Ce fil conducteur permettra à l'ENIM de maintenir une activité de recherche à haut niveau s'appuyant sur le réseau de relations internationales du LAMIH.

La collaboration sera jalonnée de plusieurs diplômes universitaires incluant une thèse en cotutelle mais aussi deux habilitations à diriger des recherches. 